\title{
WestVirginiaUniversity
}

THE RESEARCH REPOSITORY @ WVU

Graduate Theses, Dissertations, and Problem Reports

2021

\section{The Appalachian Medical Student Experience: A Case Study}

Jason Scott Hedrick

West Virginia University, jhedric8@hsc.wvu.edu

Follow this and additional works at: https://researchrepository.wvu.edu/etd

Part of the Appalachian Studies Commons, Higher Education Commons, Medical Education

Commons, and the Rural Sociology Commons

\section{Recommended Citation}

Hedrick, Jason Scott, "The Appalachian Medical Student Experience: A Case Study" (2021). Graduate Theses, Dissertations, and Problem Reports. 10155.

https://researchrepository.wvu.edu/etd/10155

This Dissertation is protected by copyright and/or related rights. It has been brought to you by the The Research Repository @ WVU with permission from the rights-holder(s). You are free to use this Dissertation in any way that is permitted by the copyright and related rights legislation that applies to your use. For other uses you must obtain permission from the rights-holder(s) directly, unless additional rights are indicated by a Creative Commons license in the record and/ or on the work itself. This Dissertation has been accepted for inclusion in WVU Graduate Theses, Dissertations, and Problem Reports collection by an authorized administrator of The Research Repository @ WVU.

For more information, please contact researchrepository@mail.wvu.edu. 
The Appalachian Medical Student Experience: A Case Study

\author{
Jason S. Hedrick
}

\begin{abstract}
Dissertation submitted to the College of Education and Human Services at West Virginia University in partial fulfillment of the requirements for the degree of Doctor of Philosophy in Higher Education

Erin McHenry-Sorber, PhD, Chair

Scott Cottrell, EdD

Rodney Hughes, PhD

Nathan Sorber, PhD

Department of Curriculum and Instruction/Literacy Studies
\end{abstract}

\title{
Morgantown, West Virginia 2021
}

Keywords: higher education, Appalachian medical students, aspiration, habitus, first-generation students, cultural capital, social capital, cultural association, gemeinschaft/gesellschaft, rural upbringing, rural practice, rural physicians, rural clinical rotations, healthcare provider shortage

Copyright 2021 Jason S. Hedrick 


\section{ABSTRACT \\ The Appalachian Medical Student Experience: A Case Study}

\section{Jason S. Hedrick}

The Appalachian region is a rural swath of mountainous terrain home to a historically distinct culture. The region's population suffers from a multitude of health issues and disparities. Notably, the region also experiences a major healthcare provider shortage despite the fact that states, like West Virginia, produce per capita, a high volume of physicians. Appalachia, and particularly West Virginia, also suffers from a number of educational disparities, which culminates into low numbers of college graduates within the population. There is a plethora of research that has explored the first-generation college student, students from rural and Appalachian backgrounds, first-generation and rural students who go to medical school, and what factors ultimately motivate a physician to choose practice in a rural locality. However, there is a dearth of literature related to the experience of medical students from Appalachia. This project begins filling that chasm. This case-study utilizes a constructivist epistemology and leverages semi-structured interviews of ten final year medical students at one medical school. All medical students in this study spent formative years in Appalachia. The researcher employed open-coding techniques and NVivo software to analyze and organize collected data.

The project includes three research questions. The first two questions relate to how relationships with home and family change throughout the students' academic journey to and through medical school and what serves as motivators or barriers for student academic success. The two questions are analyzed through Bourdieu's habitus. Students in this study largely recognized a close association with family and tight social circles in their home community. Moreover, they expected, from an early age, to go to college. While many felt support for college, it was not uncommon to face questioning parents or occasional derisive attitudes from extended family when considering medical school. Moreover, students struggled to access reliable resources in their community, including physicians. Despite these challenges, students in this study were well positioned to connect with and treat patients in the clinical phase of the curriculum, in-large part due to reconnecting with cultural associations.

The third question relates to motivations and barriers to staying and practicing in Appalachia and West Virginia. This question is explored through the lens of Tönnies' gemeinschaft/gesellschaft. Students regularly feel pulled in two directions, such as returning home to serve their local community, or at least staying in the region, while also feeling the allure to seek specialties and opportunities in population centers and medical centers outside of Appalachia. On one side, students are drawn to a familiar rural lifestyle and tight family bonds versus pursing medicine elsewhere, which include personal and professional benefit. Graduating medical students from Appalachia have spent their life working toward attaining the medical doctorate, and ultimately they are left to decide what they will do next, which may include leaving Appalachia altogether. 


\section{Contents}

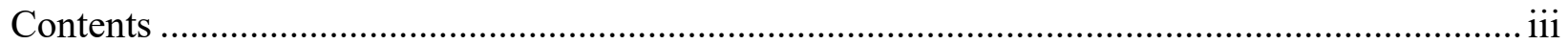

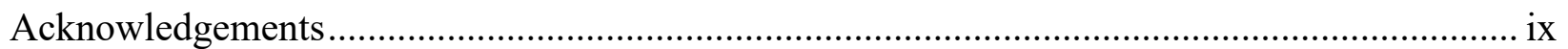

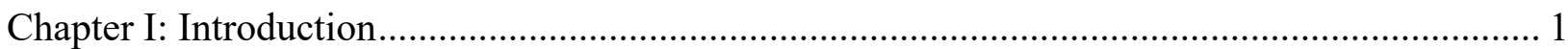

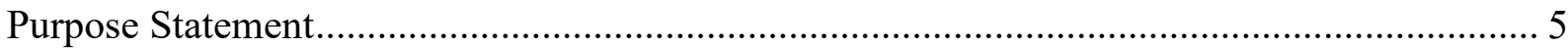

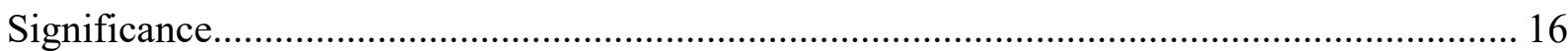

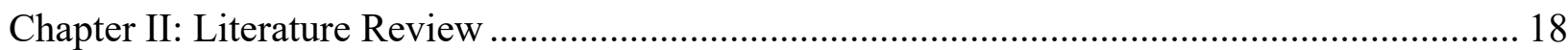

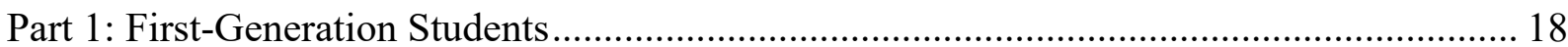

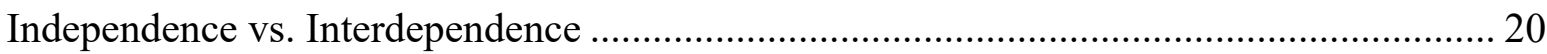

The College Track: Gatekeepers and Barriers .................................................................. 23

Self-Efficacy of first-generation students .............................................................. 26

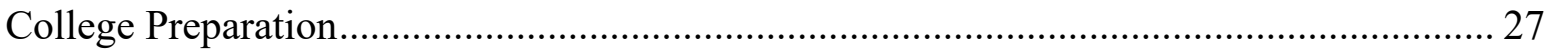

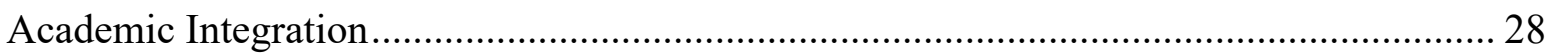

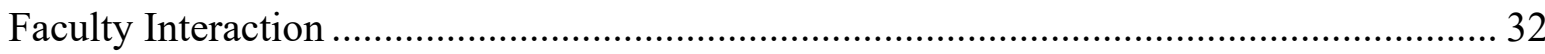

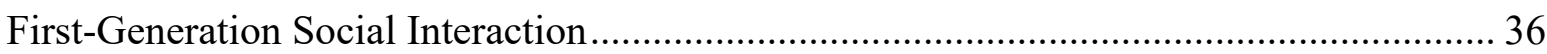

Time Commitment, Employment, \& Wellbeing............................................................ 38

The Role of Family ............................................................................................ 42

Strengths and Class Movement............................................................................ 49

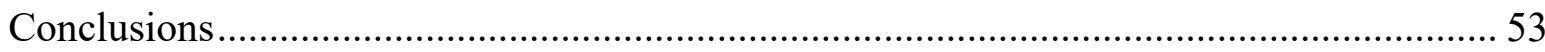

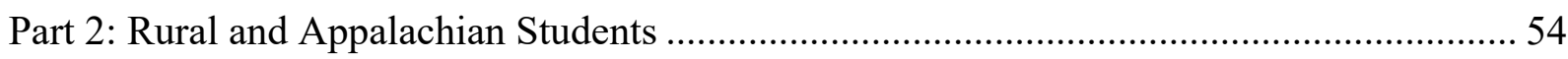

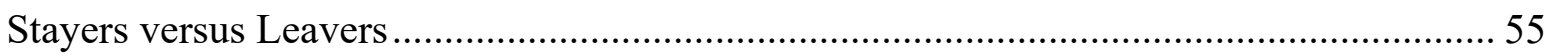

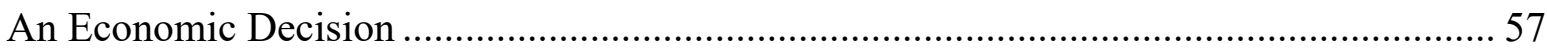

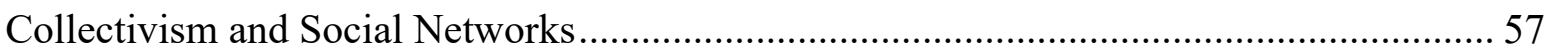

Educators, Resistance, and Value in Rural Communities............................................... 60

Self-Efficacy Among Rural Students \& Preparation for College...................................... 64

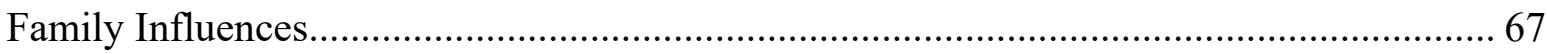

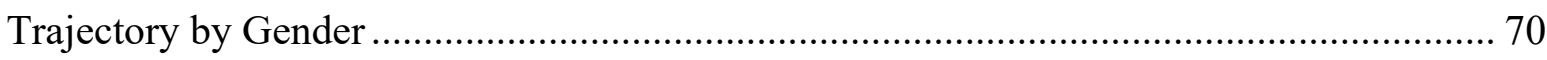

Aspirations, Identity, \& Obligations to Work............................................................... 76

High Achievers \& Community Attachment .............................................................. 79

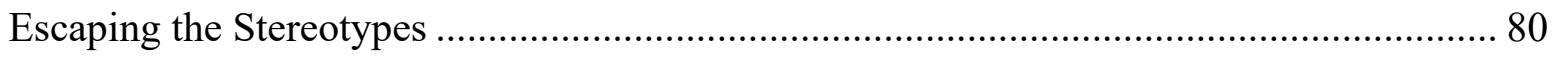

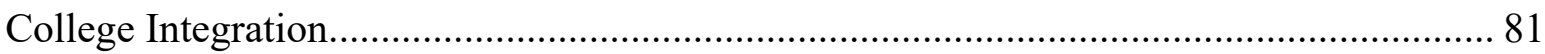

Perceptions of Preparation \& Persistence ................................................................... 88

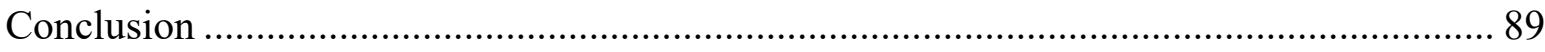

Part 3: Motivations for Rural Practice ..................................................................................... 90 


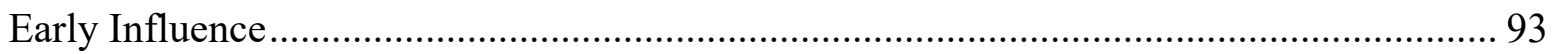

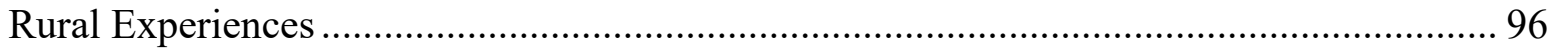

Educational and Clinical Benefits versus Drawbacks of Rural Experiences...................... 99

Rural Backgrounds versus Rural Experiences ............................................................. 103

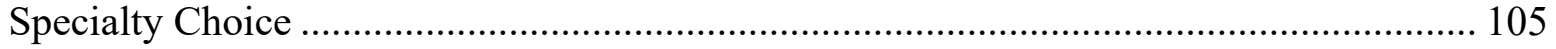

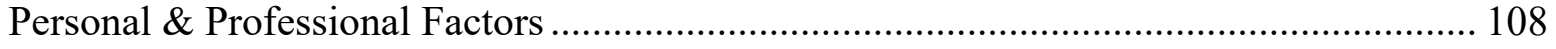

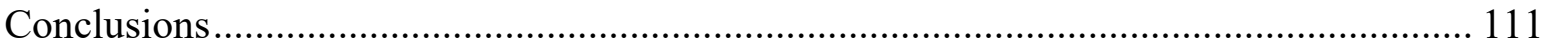

Part 4: Medical Students from First-Generation Backgrounds........................................... 112

The Path to Medical School for First-Generation and Low SES Students ....................... 113

The Transition to Medical School for First-Generation Students................................... 117

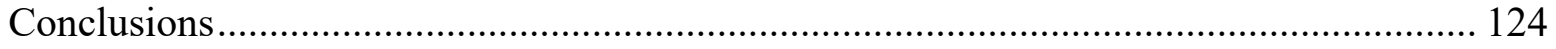

Overall Literature Review Conclusions...................................................................... 125

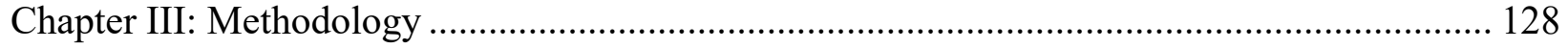

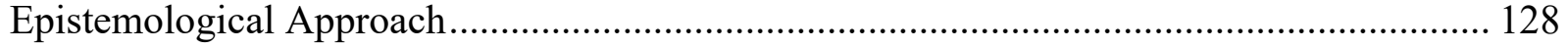

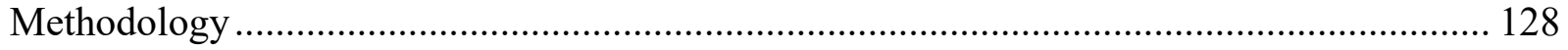

Background and Desired Study Participants.............................................................. 129

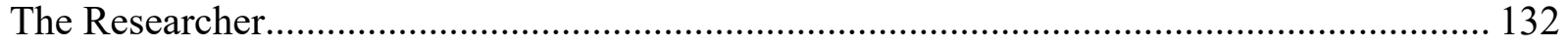

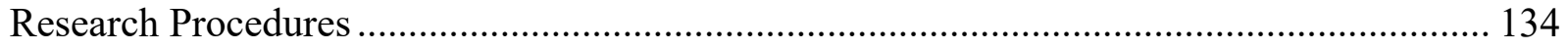

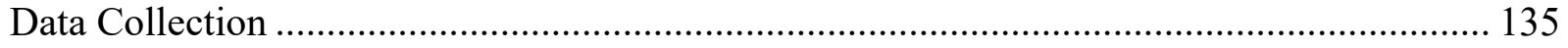

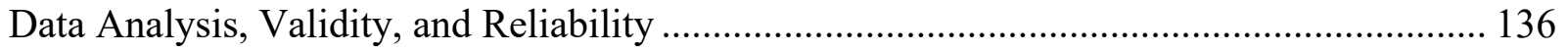

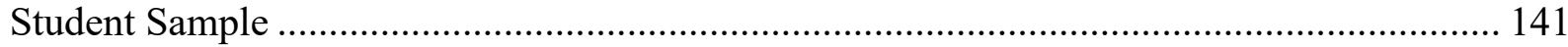

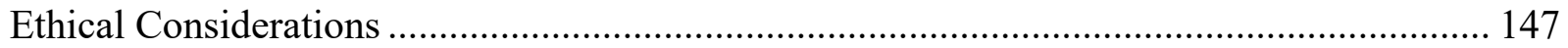

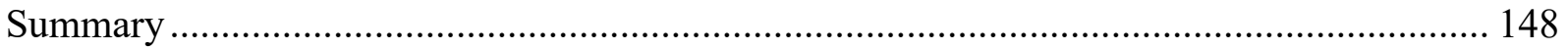

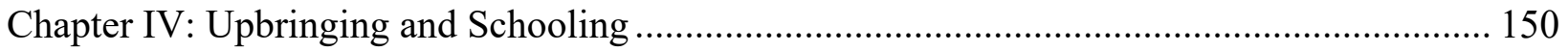

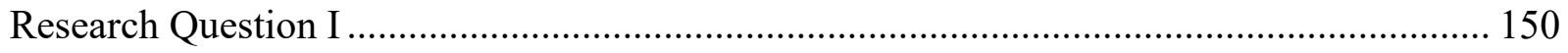

Theme 1: Family, Community, \& Land Associations Remain Important........................ 154

Theme 2: Cultural Associations Resurfaced During Rural/Community Rotations ........... 162

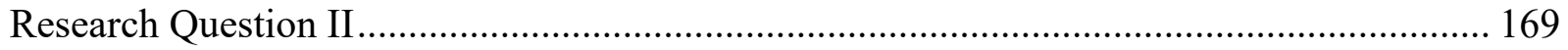

Theme 1: Facilitator: Parents and Family Members..................................................... 169

Theme 2: Facilitator: Positive Interactions with Science and Medicine.......................... 173

Theme 3: Tension: Pre-College Educational Opportunities ......................................... 177

Theme 4: Impediment: Questioners ...................................................................... 183

Theme 5: Facilitator: Themselves \& Self-Motivation .................................................... 185

Theme 6: Facilitator and Impediment: Community and Rural Physicians....................... 187 


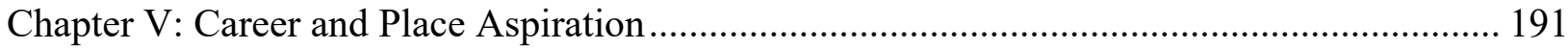

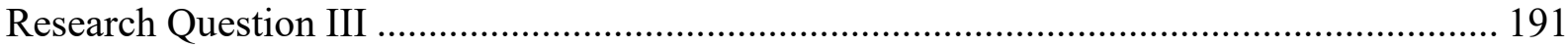

Theme 1: Motivation to Stay: Characteristics of Rural Lifestyle ......................................... 191

Theme 2: Motivation to Stay: Desire to Serve or Give Back …………………………...... 193

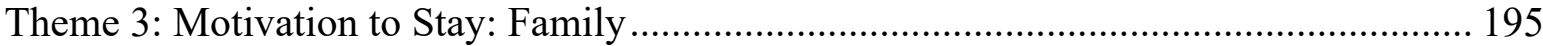

Theme 4: Motivation to Leave: The Prospect of Returning …………………………….... 198

Theme 5: Limited Direct Influence: High Achievement ..................................................... 201

Theme 6: Tension: Career Implications and Perceptions ................................................... 206

Theme 7: Motivation to Leave: Opportunities, Amenities, and Socialization Elsewhere.. 213

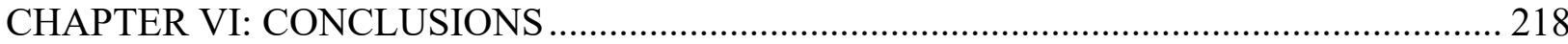

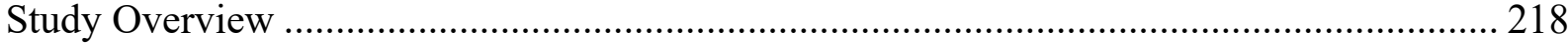

Theoretical Analysis: Habitus \& Capital ........................................................................... 218

Upbringing, Family, \& Educational Opportunities........................................................... 219

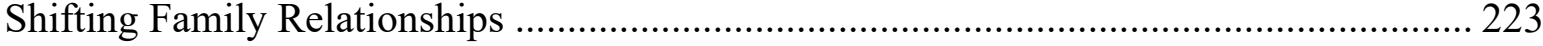

Seeking Medical School Guidance: Parents \& Family .................................................... 224

Seeking Medical School Guidance: Mentors and Physicians............................................ 226

Available Cultural \& Social Capital ................................................................................. 230

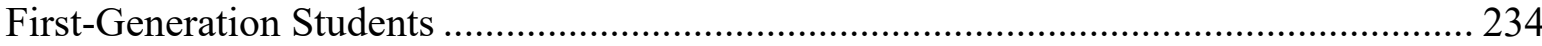

Habitus Limitations and Future Research....................................................................... 240

Theoretical Analysis: Gemeinschaft and Gesellschaft ......................................................... 241

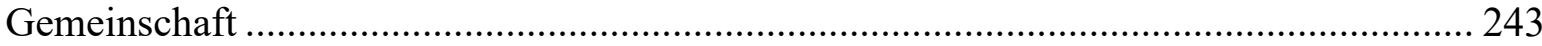

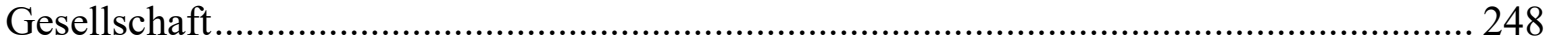

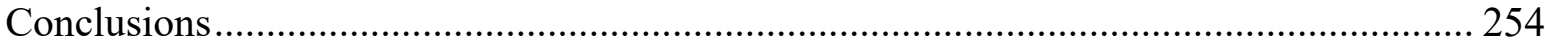

Gemeinschaft \& Gesellschaft: Future Research ……………………………………........ 255

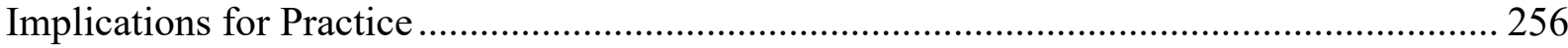

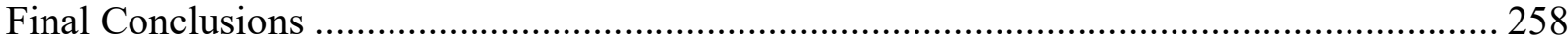

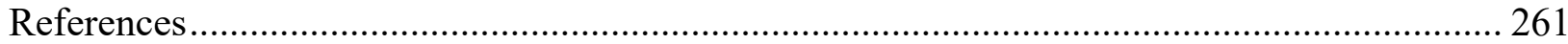

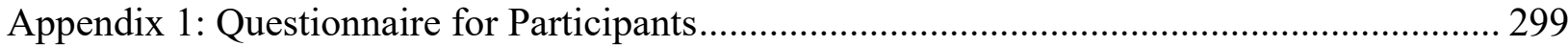

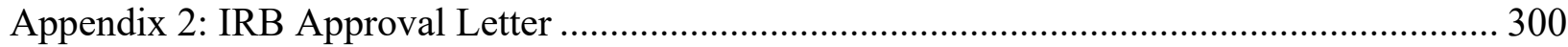

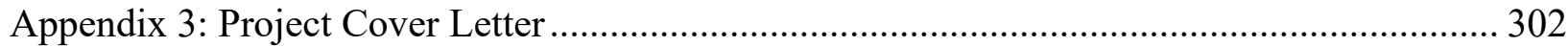

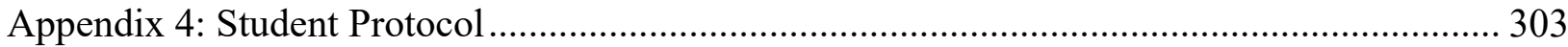


I have traveled outside the mountains, but never lived apart from them. I always feared mountains would be as jealous, as unforgiving, as any spurned lover. Leave them and they may never take you back. Besides, I never felt a need to go. There is enough to study in these hills to last a lifetime.

-Denise Giardina, Storming Heaven (1987, p. 89)

The point of my work is to show that culture and education aren't simply hobbies or minor influences. They are hugely important in the affirmation of differences between groups and social classes and in the reproduction of those differences.

-Pierre Bourdieu (Eakin, 2001) 
This dissertation is dedicated to the memory of the late Evangeline Mae Johnston, the first person who told me I was Appalachian. That moment was more profound than she or I would know, starting a line of inquiry that has continued to grow over the years. 


\section{Acknowledgements}

I have been privileged to be influenced and academically invigorated by a number of colleagues and mentors. First, I would be remiss to not mention my first colleagues, Elizabeth McMullen, Joseph Phillips, and Dr. Joseph Rizzo, as well as my first graduate school advisor, Dr. Melissa Bingmann. Each of you supported and contributed to me developing confidence in my own thoughts. Thank you.

I would like to thank committee members, Dr. Rodney Hughes and Dr. Nathan Sorber. You both exhibit excellence as scholars and have created a positive environment of inquiry. Because of this atmosphere, I have also felt encouraged and energized to ask my own questions, find my own answers, and build my own arguments. Thank you.

To my committee chair, Dr. Erin McHenry-Sorber, I cannot thank you enough for the time and insight you have provided throughout this project. I have felt nothing but encouraged to keep going and keep refining my work. I am sincerely grateful for your mentorship. Thank you.

My medical education colleagues have afforded me with many opportunities to learn and grow as an individual and professional. Particularly, I would like to thank Dr. Anna Lama for continued support. Also, Dr. Norman Ferrari, who has always seen my potential, long before I see it in myself. And, finally, committee member Dr. Scott Cottrell, who has served in a number of roles including advocate and mentor. The three of you have shaped how I view and grapple with both intellectual and practical problems. Thank you.

I would also like to thank Dr. Larry Rhodes, an individual who is truly emblematic of the Appalachian physician. Your experiences, knowledge, and how you relate them into lessons about Appalachia are invaluable. Thank you.

Aside from my colleagues and mentors, I would also like to specifically thank my family. To Jennifer, Scott, Eva, Hallie, Dan, Terry, Andrea, Phillip, Joe, and Shannon, there were many evenings and Saturday afternoons that I was busy typing downstairs missing valuable moments with you all, and amazingly, you have all understood. I am looking forward to spending a lot more time together. Thank you.

To Mom and Dad, Rick and Debbie Hedrick, I can undoubtably say that my academic success is largely attributed to your love and support over the years. I, myself, was a rural Appalachian kid and you always had dreams that your children would make the most of every opportunity they were given. Those dreams have made all the difference. Thank you.

Finally, to my wife, Dr. Lindsay Hedrick, your decision to ultimately go back to school was motivating and inspiring. You always lead the way in all things that you do, and it was no different for this endeavor! Your unwavering support, understanding, and everyday love has once again made our dreams attainable. Thank you. 


\section{Chapter I: Introduction}

Appalachia is both a geopolitical region and home to a distinct culture historically mired in a number of deep-seated social disparities. ${ }^{1}$ Those disparities have resulted in, among other things, poor educational attainment, higher risk for preventable disease, and insufficient healthcare access stemming from provider shortages (Appalachian Regional Commission [ARC], 2017). Notably, healthcare access is disproportionality limited in Appalachia compared to the rest of the nation (Marshall et al., 2017). There are approximately 75.6 primary care physicians for every 100,000 in the nation's population, but only 66.8 in Appalachia (Marshall et al., 2017). In central Appalachia, that number dwindles to a mere 50.9 —a third fewer physicians per 100,000 individuals (Marshall et al., 2017). ${ }^{2}$ By comparing rural Appalachia and non-rural Appalachia, rural counties average 55.6 primary care physicians per 100,000 (Marshall et al., 2017). In economically distressed counties in Appalachia, that number declines to 40.6, which approaches half of the national average (Marshall et al., 2017). For specialists, the data are just as bleak. While there are 153 specialists per 100,000 in the nation, in Appalachia there are 110 per 100,000, 64 per 100,000 in rural Appalachia, and 28 per 100,000 in economically distressed

\footnotetext{
${ }^{1}$ This project relies on two definitions of Appalachia. First, Appalachia is a physical place. The Appalachian Regional Commission (ARC) has defined the Appalachian region as 420 counties, including all West Virginia counties and parts of thirteen states from southern New York to northern Alabama, Georgia, and Mississippi (ARC, n.d.b.). The ARC's definition relies on geographic and natural features as well as socioeconomic considerations. This is a constructed definition beneficial as a reference point. A second matter needing to be addressed is the concept of a sociocultural definition of Appalachia. Raitz et al. (1984/2018) astutely compile a number of Appalachia definitions that are quite varied in the finite boundaries of what constitutes Appalachia. Even within the "boundaries," there are further subsets, regions, and definitions that seem to further qualify an association of not only the binary concept of Appalachian versus non-Appalachian, but also what degree and locality of association. Individual and group relationship to the region and culture is an important concept for this project. Therefore, when referencing Appalachia in this project there are two concepts. Foremost, Appalachia is the somewhat liberally applied 420 counties as designated by the ARC. However, when referencing Appalachia in the cultural context, the definition is more elusive and ill defined. It is reductive to evenly apply a sociocultural definition to Appalachia and Appalachians, as there are degrees of association and self-identification. Therefore, when referencing Appalachian(s), the general intent is to include the population within the physical region, while acknowledging that every individual will have a varying degree of cultural affiliation.

${ }^{2}$ The ARC (n.d.b.) defines central Appalachia as including seven counties in southern West Virginia, seven counties in southwestern Virginia, all of Appalachian Kentucky, and 14 counties in northeastern Tennessee.
} 
counties (Marshall et al., 2017). Due to the shortage, many patients turn to primary care physicians, who are of course already stretched thin. Finally, and of equal importance, the region suffers from mental healthcare shortages. Nationally, there are about 201 mental healthcare providers per 100,000 in the US compared to 130 across Appalachia, and 123 in Appalachian distressed counties (Marshall et al., 2017).

The aforementioned provider shortages are further troubling when considering the implications for the population. The region suffers from a multitude of health and healthcare issues, which in many instances are significantly worse than the rest of the nation. Of the 10 leading causes of death in the US, Appalachia has a higher mortality rate in seven of those areas, including cancer, chronic obstructive pulmonary disease (COPD), diabetes, heart disease, injury, stroke, and suicide (ARC, n.d.a.). The region, but particularly central Appalachia, has suffered from a major drug epidemic — specifically opioid abuse, which has contributed to a higher rate of death from poisoning (ARC, n.d.a.). Regarding general wellbeing, again Appalachia lags well behind optimal levels with greater numbers reporting physically and mentally unhealthy days including a greater frequency of depression (ARC, n.d.a.). Of all the aforementioned health issues facing the region, many are predisposed to poor health with greater instances of obesity, tobacco use, and general inactivity (ARC, n.d.a.).

The chronic healthcare disparities and prevalence of preventable disease are issues that impact what the Centers for Disease Control and Prevention (Centers for Disease Control and Prevention $[\mathrm{CDCP}], 2014)$ identify as determinants of health. Determinants of health relate to factors that generally increase or decrease the quality of life for individuals and populations (CDCP, 2014). These factors include social contexts, access to healthcare, economic stability, education, and the local environment (CDCP, 2014). Although each determinant and challenge 
in Appalachia could be discussed at great length, education and economic stability, in particular, have been major issues for the region. Appalachia remains behind the nation in higher education attainment. While close to one-third of the American population attains at least a bachelor's degree, only about a quarter of Appalachian adults ever achieve that level of education (ARC, 2020). In central Appalachia, bachelor degree attainment is less than $15 \%$ of the adult population (ARC, 2020). Regarding economic stability, between 2015 and 2017, the entire US averaged a 4.8\% unemployment rate, while Appalachia's rate was slightly higher at 5.4\% with areas like Appalachia Kentucky over 7\% and West Virginia (WV) around 6\% (ARC, 2019). During this same period, the poverty rate for the entire country was $14.6 \%$, but averaged $16.3 \%$ in Appalachia, and peaked as high as 25\% in central Appalachia (ARC, 2019).

The discussion of social determinants of health points to the fact that these issues are compounding and circuitous. Together, they create a vicious cycle - a wicked problem of sorts that cannot be attributed to any single factor. For example, fewer physicians impact access, and access dictates overall disease prevention, management, and outcomes. Poor outcomes contribute to the overall makeup and ability of local and regional economies. Poor economics and health disparities also influence other determinants including access to education, the overall environment, and other social contexts that reinforce and normalize circumstances. This explanation is simplistic, but overall representative. It is not to say that access to healthcare is driving the disparities in Appalachia, but instead, it is one factor in the cycle that influences other determinants. Practitioner shortage, healthcare access, and health outcomes are spokes of a damaged wheel. The problems are complex and chronic, and many supposed solutions fail to fix that wheel. Instead, solutions are temporary and insular, failing to remedy chronic challenges. 
However, lasting and informed solutions do have the potential to reinforce positive change, redirect determinants of health, and strengthen this proverbial wheel.

WV has attempted to alleviate healthcare disparities and practitioner shortages, partly through recruitment of medical doctor trainees from within the region. In fact, among the state's three medical schools, WV trains 89 medical students per every 100,000 in the state's population - the greatest number of trainees, per capita, in the nation (Association of American Medical Colleges [AAMC], 2019b). For reference, the national median among states is 37 students per every 100,000 in the population (AAMC, 2019b). Aside from WV, all states with counties in the central Appalachian region, save North Carolina, train physicians at a higher rate than the national state median (AAMC, 2019b). Moreover, among students from WV who matriculated to a medical school somewhere in the country, $88.9 \%$ of students from WV actually stayed in WV to study, making it fourth in the country for greatest number of residents retained to a medical school within their state (AAMC, 2019b). However, the retention of medical school graduates to remain in WV has been lackluster. The national median for remaining in the same state the student graduated medical school is about $40 \%$; meanwhile, only about $30.4 \%$ of graduating physicians from a WV medical school remain in the state, ranking $35^{\text {th }}$ in the country (AAMC, 2019b). In other words, WV suffers from medical student "brain drain," training the greatest per capita number of medical students, most of which come from in-state, only to see nearly $70 \%$ leave upon graduation.

Some medical schools in the region, such as West Virginia University (WVU), recruit students from Appalachian counties (Diversity Policy, 2019). Reasoning for this recruitment has largely been twofold. The first is simply that much of the Appalachian region and WV counties have low educational attainment (Pollard \& Jacobsen, 2019). Some of the educational disparities 
have resulted directly from persistent economic instability, a shortage of qualified educators in underserved areas, and an inflexibility to shift toward new job creating industries (Wright, Cunningham, \& Strangle, 2016). With these challenges, the logic for recruitment is that this rural population of students should be given the opportunity to contribute to the medical field. There are capable students from rural areas, but many have not met their full academic potential. A second motive is the hope that some students, with a familiarity and ties to the culture, will eventually stay in the region and serve destitute communities. Although schools have theoretically taken steps to enhance the likelihood of physicians staying in state through means like required rural rotations or optional rural medicine tracks, retention has still not met levels to ameliorate these shortages and disparities. In sum, although there are a multitude of problems at play, an issue worth more closely examining is the recruitment of students from Appalachian counties and their failure to remain for medical practice.

\section{Purpose Statement}

The purpose of this study is to understand the transition and associated challenges that students from Appalachia experience while moving through the medical education experience. The recruitment of Appalachian students and their somewhat failed retention to practice in the region has created an identifiable yet understudied phenomenon and problem. The state and region are both troubled by a provider shortage and the related social determinants of health. Moreover, the experience of this student population is vastly understudied with the literature failing to investigate Appalachian students in medical education from either first-generation or continuing generation backgrounds. ${ }^{3}$ Medical students and future physicians, particularly those

\footnotetext{
${ }^{3}$ The Association of American Medical College's (AAMC) American Medical College Application System (AMCAS) is a common system utilized nationally by most allopathic medical schools for admission processing. In 2018, the system initialized a first-generation student indicator that, based upon applicant responses, designates a student as first-generation if applicable (AAMC, 2017). To qualify for this designation,
} 
from first-generation college families in Appalachia, are upwardly mobile and have a newly enhanced agency over their future. The transition, then, brings into question what other changes these students are experiencing. The literature has explored the separation of cultures and subsequent challenges that both Appalachian and first-generation or low-SES college student's experience upon leaving home, but has stopped short of fully exploring the experience of medical students. Therefore, guiding research questions include:

1. How do Appalachian students interpret their relationship with home-including cultural associations - as they transition through the medical education program?

2. Within this student population, who or what do they recognize as facilitators or impediments to their academic journey?

3. What motivates some students to stay or leave Appalachia or WV?

The specific pragmatic reason that a student chooses not to return to their community or rural Appalachia is likely multifactorial. Residency training is assuredly a choke point in the quest to become a licensed and board-certified physician. Across the nation there are more than 137,000 active residents and fellows and about 120,000 enrolled medical students - a ratio of 1.1 (AAMC, 2019b). In West Virginia, there are 920 residency or fellowship positions to 1,611 actively enrolled medical students - a ratio of 0.6 (AAMC, 2019b). As a result, WV ranks $43^{\text {rd }}$

applicants may not have a parent who has received an associate's degree or greater. This definition of being a first-generation student is utilized for this research. However, it is important to recognize subtleties surrounding this overarching definition. For example, a student with parent(s) who completed a technical program with an associate's degree may still consider themselves a first-generation college student. Those nuances will be considered on a case-by-case basis with respect to how their perspective may or may not add to the research here. Moreover, there are inconsistencies across the literature regarding the characterization or categorization of first-generation college students. As will be seen in this section and the next, some authors used varied or augmented definitions. Regarding a few pieces of literature germane to this study, some researchers, particularly from Australia, Canada, and the United Kingdom commonly reference this population as first-in-family. Others utilize terms like "working class," which is not necessarily equal to a first-generation background, but perhaps common in certain cultures or vernaculars. Nonetheless, some of this literature is relatable to matters here. When differences in definition surface, they are noted throughout. 
in the nation for undergraduate medical education (UME) positions versus graduate medical education (GME) positions (AAMC, 2019b). Examining the ratio between the two does not necessarily equate to a clear picture of annual availability as residency programs are of varied length, but it does present a disparity. To elucidate the point further, certain specialties in the state are highly competitive. For example, the nation has more than 1,400 active dermatology residents - a highly selective specialty (AAMC, 2019a). WV has a single dermatology program, which accepts two residents annually. In sum, the state, for reasons too protracted to explore here, is underserved by both GME positions and depth for the volume of UME learners.

Aside from the plight of too few residency positions in the state, modern medicine in general has shifted to a systems-based practice approach. Practicing providers no longer work in privately owned clinics but rely on an integration with a healthcare system. In turn, the era of private practitioners has dissipated. Robust residency and fellowship program offerings (i.e., specialty training) as well as health systems in general are often associated with larger population centers (Marshall et al., 2017). Many community clinics and hospitals may be affiliated or partnered with larger hospital systems, and association serves as a pipeline for patients to access system-based offerings (e.g., specialists) (Marshall et al., 2017). Despite perhaps good faith efforts to connect services to local communities, patients from the most rural areas must still interface with these large population centers and hospitals for certain types of care. Again, accessibility remains the pressing issue with destitute populations facing distance as well as economic limitations as major hurdles. Therefore, systems-based practice has, in some respects, further pulled providers and residents to population centers. Moreover, in an effort to improve access in local communities, the transition has presented its own set of challenges. 
Other literature has surveyed how to retain medical students for rural practice once they have entered the curriculum. Curran and Rourke (2004) found that students from rural areas (not specific to Appalachia) and interested in the family medicine discipline were most likely to practice rural medicine. However, the authors also found that simply being from a rural area was less of a factor for practicing in a rural community (Curran \& Rourke, 2004). Medical schools did not always recruit (or recruit effectively) from rural areas, leading to fewer students who may be interested in rural practice based on their own upbringing. Barrett et al. (2011) conducted a meta-analysis to ascertain if required rural experiences in medical education influenced students' decision to practice in a rural area. They note, "Although the evidence supports that these rotations influence practice site and career choice, it is not clear whether they reinforce preexisting interest or have the ability to motivate previously uninterested students to consider careers in primary care or rural medicine" (p. 259). Regarding rural tracks, Roseamelia et al. (2014) found in a qualitative study that students in a rural health track were not so much committed to the idea of rural practice, but were simply less interested in urban practice.

Despite student recruitment efforts and attempts to promote rural practice, there are also significant systemic and social barriers for students to even have the opportunity to seek a medical education, particularly in a region like Appalachia. As previously mentioned, educational disparities in Appalachia abound. First, because the number of students who attend and complete college is less than the national average, there are, of course, more first-generation college students. The literature shows that the first-generation students experience higher education differently than their peers. Ali and Saunders (2006) argue:

The low percentage of adults holding college degrees means that Appalachian youth are exposed to fewer role models with postsecondary education and have diminished access 
to information and fewer supports for postsecondary educational opportunities, which can potentially result in low confidence in their academic abilities. (p. 39)

In fact, confidence in academic ability is a common theme in a number of pieces relating to Appalachian students' desire to attend or complete college. Wood (2012), who explored influences for attending college among Appalachian students found that students cited faith in the own academic abilities as a reason to actually attend college. This belief possibly signals that perhaps college was not a compulsory route, but was instead reserved or regarded as a path for only the seemingly best and brightest in the community.

Much of the concept of confidence resonates from familial support. Family ties and acceptance in Appalachia are important to the culture. At the heart of the matter is that Appalachia rests just outside of mainstream middleclass America- to which the infrastructure of higher education closely aligns. Wallace and Diekroger (2000) denote that by surveying 127 college students (91\% considered Appalachian), many students:

Reported being expected to do extra work that kept them from their schoolwork. Other frequent responses included being accused of "acting better" than others who did not attend college, being ignored when talking about college experiences, being told to not waste time on college, receiving dirty looks when talking about college, and being told that they would never complete a degree. (p. 1)

Moreover, the authors found that these negative messages or connotations regarding higher education came directly from family members (Wallace \& Diekroger, 2000).

In fact, association and support from family is recurrent throughout the literature.

Chenoweth and Galliher (2004), after investigating the college aspirations of rural West Virginia high school students, found that family and peer aspirations were some of the greatest influences 
on the individual student. Particularly, the authors argue that having a father who attended college or supported their child in attending college directly correlated to the student's desire to attend college, particularly among males (Chenoweth \& Galliher, 2004). The support of family and the community was also a factor that Hlinka (2017) describes as having an impact on community college persistence in Appalachia. The author notes that two of three factors include the "community's and family's values of education provid[ing] the essential push to attend and complete college... [and] overcoming the pull of family obligations..." (Hlinka, 2017, p. 144).

Briggs (2010) specifically examined the perceptions of first-generation Appalachian college students toward higher education. The author denotes heritage as a major theme in students making sense of their new environment and the college experience (Briggs, 2010). ${ }^{4}$ More specifically, heritage was associated with a divide that is established by going to college and leaving behind family, which represents a cultural association (Briggs, 2010). Beasley (2011), through an ethnographic study of first-generation Appalachian students highlights this tension exceedingly well. The author pulls from and cites a concept_ " "moving up implies moving out" originally put forth by Hektner (1995) (Beasley, 2011, p. 212). Many students "hold conflicting views - both a desire to leave and remain... One fourth-year student described the necessity of "letting go of home" to be able to make it at her university, an example of the perceived choice students face between family/home and leaving” (Beasley, 2011, p. 234-

\footnotetext{
${ }^{4}$ Briggs (2010) does not explicitly define the term "heritage." However, the author denotes that "Heritage plays an important role in the way the students in this study perceive their college experience even though a majority of the students ignored the term, Appalachia, as part of their heritage. For these students heritage is defined primarily through the lens of family and home culture. They shared stories of their parents and grandparents, and they talked about cultural aspects of their lives that they brought with them to ETSU [East Tennessee State University], including food and music, that they describe as uniquely Appalachian" (p. 103). The author continues by stating that the concept of heritage is closely related to the second theme of the study, proximity (Briggs, 2010). Proximity typically meant how close the college was to the student's home and family.
} 
235). For many first-generation Appalachian students, moving up denotes an opportunity for higher education and career paths, but moving out of the culture.

Underpinning much of this discussion related to both Appalachian and first-generation students' social mobility are concepts that should, at the very least, be touched upon. Glass (1954/1998), in a look at post-World War British society, argues that educational reform and expanded access for the masses allowed more individuals to aspire greater social distances (Glass, 1954/1998). The expansion of social mobility, Glass (1954/1998) explicates, allowed individuals to better align to the skills, aptitudes, or expertise of positions and less as a result of their already allotted social position. Glass (1954/1998) proverbially intimates, "fewer square pegs in round holes" (p. 25). A better aligned occupation to the individual allows for greater job fulfillment and ultimately worker efficiency. Relatedly, at a macrolevel, the economy and social structures are nimbler and more readily subject to change and evolution (Glass, 1954/1998). The author goes on to explicate that even a feeling of mobility or opportunity that may be much more limited in actuality likely merits similar positive results (Glass, 1954/1998). For example, American society benefits from ideals of these concepts (i.e., access to opportunities), but in actuality opportunity across classes may be quite inequitable (Glass, 1954/1998).

A final point to be made regarding Glass' (1954/1998) discussion of class particularly germane to this study relates to a paradox resulting from maintaining or progressing social class standing-i.e., expanding social mobility. Particularly among middle class families, there is a desire to preserve or progress standing to the point that parents sacrifice great deals for their children's education and success. The concern that a family or its offspring may slip or fail to advance social standing is enough to create a level of anxiety among members of the class (Glass, 1954/1998). In turn, "actual movement itself may, save in special circumstances, distort 
or destroy kinship associations, with possible personal and social deprivation" (Glass, 1954/1998, p. 25). While enhanced social mobility generates benefits, with the greatest proportion to those at the lowest social station, Glass (1954/1998) warns of the potential for generational divides.

Glass' (1954/1988) foundational work is important for understanding some of the fundamental benefits and challenges of social mobility. Boudon (1974) however, takes a specific look at the relationship between educational and social opportunity, and that educational opportunity is not necessarily synonymous with social opportunity (Boudon, 1974). Even though there may be increasing educational opportunity in Western society, Boudon (1974) takes issue with the notion that education is a tool to ascertain and develop levels of competency, which in turn can group students into vocational trajectories. This idea, he contends, would imply an even playing field for students to engage those educational opportunities, which would then lead to the concept that any career was possible from the standpoint of accessing the education requirements (Boudon, 1974). Moreover, Boudon (1974) challenges the assumption that perhaps one reason that individuals from the lower bounds of society do not engage education is because it is undervalued-i.e., not consistent with cultural values. Instead, reasons for seeking or not seeking these experiences should be looked at through "decision fields that are a function of social position" (p. 197).

Boudon (1974) goes on to note that as opportunity for education expands, the demand for greater levels of education will also increase generation by generation. Therefore, as access expands, the social advantage of the education for each individual is diminished even though society, in general, still receives the benefit of increased education and knowledge (Boudon, 1974). Therefore, this aggregation paradox, as he notes, intimates that greater access to education 
does not automatically compute to greater social opportunity (Boudon, 1974). As educational opportunity leads to more individuals seeking that education, the next level of potential educational opportunity is then layered on to the former, elongating or making more complex the educational experience required or expected to meet certain social opportunities.

Returning to Boudon's (1974) notion of decision fields, this idea can be expanded by considering the positions of Bourdieu and his postulation of habitus (Bourdieu, 1984; Maton, 2012). The concept of habitus is that all individuals have a history, and that history has been shaped by experiences and surroundings. Maton (2012) summarizes:

Habitus focuses on our way of acting, feeling, thinking, and being. It captures how we carry within us our history, how we bring this history in our present circumstances, and how we then make choices to act in certain ways and not others... Where we are in life at any one moment is the result of numberless events in the past that have shaped our path. We are faced at any moment with a variety of possible forks in that path, or choices of action and beliefs. This range of choices depends on our current context,... but at the same time which of these choices are visible to us and which we do not see as possible is the result of our past journey. (p. 51)

Bourdieu (1984) further posits that:

The habitus is not only a structuring structure, which organizes practices and the perception of practices, but also a structured structure: the principle of division into logical classes which organizes the perception of the social world is itself the product of internalization of the division into social classes. (p. 170)

Bourdieu analogized habitus to skills utilized while playing a game (Maton, 2012; Thomson, 2012). In certain contexts, based on past experiences, an individual will know or be familiar with 
any particular game, including its rules, at varied levels (Maton, 2012; Thomson, 2012). Other players will bring their own skills based on their past experiences, or habitus. Therefore, each person arrives at the moment or situation —-i.e., the game- - with a unique knowledge of the rules, expectations, and relatable experiences to help navigate that game. However, it is also important to remember that individual actions or decisions should not be reduced to simply being the result of association with a culture or social group (Maton, 2012). In other words, it would be inappropriate to rationalize an individual's beliefs or behavior as simply a result of affiliation or their habitus (Maton, 2012).

Habitus, and the notion of the game analogy, is an important concept when considering social and cultural capital. Students who are accustomed to the game, so to speak, may already understand some of the rules or are, more-or-less, inclined to think and act in ways that are beneficial to engagement with an environment. They also may have a proclivity to be drawn toward certain paths or expectations such as post-secondary education or career choices, because it more closely aligns with their habitus. By gaining or achieving what is important in the individual's habitus, they are essentially building or reinforcing their social and cultural capital. This is important when considering inequality. Moore (2012) notes, "The inequalities associated with cultural capital reflect inequality in capacities to acquire capital which themselves reflect prior inequalities in the possession of cultural capital" (p. 106).

The foregoing discussion of social mobility, educational opportunity, and habitus are not an attempt to fully explicate all gradations of these concepts. Instead, they are looked upon to provide a guiding framework and theoretical basis for this research. Each of the aforementioned contributions and theories add important elements of consideration to this project. Notably, Glass (1954/1988) presents the basis for social mobility and the risks of decoupling relationships and 
generations. Boudon (1974) argues that educational access does not necessarily equate to social opportunity, particularly as access and opportunity are recognized differently across society. Finally, habitus elucidates that individuals based upon origins, experiences, and opportunity will know about, and ultimately respond to, environments differently. The higher education literature is replete with commentary and research that utilize the aforementioned concepts of habitus and capital. Explicating these concepts help this research come into conversation with a handful of studies that have examined populations of medical students (e.g., Bassett et al., 2019, Beagan, 2005; Brosnan et al., 2016; Luke, 2003; Nicholson, 2013). Luke (2003) presents the concept of the medical habitus and that some students, based upon their own backgrounds and habitus, may be better prepared to take advantage of or fit into the medical environment (Nicholson, 2013). Moreover, medical students from first-generation or lower socioeconomic backgrounds bring to the environment differing forms of social or cultural capital, some of which may or may not be beneficial in the new environment (Beagan, 2005). Students from non-dominate cultures must ascertain how to manage and balance multiple identities, one that corresponds to their home or origins and one more closely related to the medical school (Bassett et al., 2019; Brosnan et al. 2016). This research aims to progress a similar line of inquiry by focusing on the experience of Appalachian medical students.

Additionally, particularly regarding the third research question and what may motivate some students to stay or leave the region or state, this research project also leverages the theoretical duality of gemeinschaft/gesellschaft originally put forth by Tönnies (1887/2001). Gemeinschaft, or community, is the idea that individuals in certain social structure or spaces may feel compelled or pulled to maintain social ties and networks for the betterment or benefit of that group (Tönnies, 1887/2001). Gesellschaft, or society, represents the greater world where 
associations are more contractual and less obligatory, and the individual has independence from maintenance of local social structures (Tönnies,1887/2001). This dichotomy is an important consideration for this project as Appalachian medical students who approach graduation must consider how their future plans, including practice location, may change or alter their associations and relationships with home.

The research employs a constructivist epistemology (Merriam \& Tisdell, 2016). Each student has their own perspectives and perceptions, which may be shaped by their immediate social constructs or locality of upbringing. The key, then, is to pinpoint unity and incongruity between their experiences. Moreover, how are those experiences influencing and shaping their perceptions of their current career trajectory? By approaching the research from the perspective of the student, it is possible to explore the most formative time in the medical education experience. The study utilizes a case-study research design that relies on semi-structured interviews of final-year medical students at West Virginia University.

\section{Significance}

The implications for this research study are twofold. First, this research adds to an existing gap in the literature as well as inform future recruitment and retention practices in not only West Virginia, but also the Appalachian region. Regarding the literature, there are studies that have targeted the experience of first-generation students in both Appalachian and nonAppalachian contexts. Moreover, some literature does specifically target first-generation experience in medical education, but there is paucity for studies that deliberately seek to understand this educational experience for Appalachian medical students. Second, this study helps inform the literature surrounding what motivates a medical student to stay or leave including how students originating from Appalachia may be pulled in disparate directions in 
light of their upbringing. Moreover, by understanding this cohort of students, there are practical considerations for recruitment and retention. Despite efforts to recruit from rural areas, practicing physicians are not always retained to these areas. Therefore, understanding student tensions and motivations can help explicate how to recruit students to practice in the state or region. In other words, what is the student perspective when engaging the multifaceted and layered challenge of physician retention? Finally, are there programs, services, or specific experiences that could be provided to medical students to encourage their willingness to practice in destitute communities? The state and region continue to suffer from a number of disparities, including educational inequalities and practitioner shortages. By better understanding what motivates a student to stay or leave, it is possible to examine this ill-structured problem from the perspective of the student including their sense of their own upbringing, motivation, tensions, and experiences. 


\section{Chapter II: Literature Review}

\section{Part 1: First-Generation Students}

Acknowledging general trends related to first-generation students is beneficial to better explicate how the literature has approached this population's experience. In the United States (U.S.), as of academic year $2015-2016,56 \%$ of undergraduates did not have a parent with a bachelor's degree, while $24 \%$ of students had parents with no postsecondary education whatsoever (RTI International, 2019b). ${ }^{5}$ Therefore, more students who engage the higher education system are doing so as family pioneers, entering unchartered waters and questing for educational credentials foreign to their immediate family (McInnis \& James, 1996; King et al., 2019). Moreover, compared to their peers, first-generation students have a number of statistical differences. first-generation students are more likely to be over the age of $30(28 \%$ of firstgeneration students versus $16 \%$ of continuing-generation students) and have dependents (Engle \& Tinto, 2008; RTI International, 2019b). Compared to continuing generation students, who are more gender-balanced (52\% female), first-generation students are more likely to identify as female (60\%), more likely to identify as black/African American or Hispanic/Latinx as opposed to Caucasian, and come from single-parent families (Engle \& Tinto, 2008; RTI International, 2019b). first-generation students are more likely than their peers to be nonnative English speakers (Engle \& Tinto, 2008).

Enrollment patterns between first-generation students and continuing-generation students are also noticeably disparate. Public two-year institutions (64\% first-generation students), private nonprofit two-year institutions (69\%), and both private for-profit two-year (70\%) and four-year

\footnotetext{
${ }^{5}$ The RTI International Fact Sheets referenced in this section uses the definition of first-generation as having no parent with a bachelor's degree or higher, while continuing generation is defined as having at least one parent who has achieved a bachelor's degree or higher.
} 
institutions (72\%) are dominated by first-generation enrollees compared to public four-year (47\% first-generation students) and private non-profit four-year (43\%) colleges (RTI

International, 2019b). Despite the fact that the majority of low-income first-generation students begin their higher education journeys at a two-year or for-profit college, they are seven times more likely to graduate with a bachelor's degree by starting at a traditional four-year institution (Engle \& Tinto, 2008). ${ }^{6}$ Financially, first-generation students come from different economical pictures with parent median annual income of first-generation students around $\$ 41,000$ compared to continuing-generation students at \$90,000 (RTI International, 2019b). Somewhat comparably, approximately two-thirds of first-generation students were employed during academic year 20152016, compared to $61 \%$ of continuing-generation students; however, first-generation students typically worked more hours - a median 20 hours per week - versus continuing-generation students - a median 12 hours per week (RTI International, 2019a). Likewise, only 65\% of firstgeneration students in their first-year of college were enrolled full-time versus $75 \%$ of continuing-generation students, which couples with a pattern of taking fewer credit hours (Pascarella et al., 2004; RTI International 2019c).

Within six-years of entering higher education, first-generation students are more likely to obtain a certificate or an associate's degree, but are far less likely to achieve a bachelor's degree

\footnotetext{
${ }^{6}$ It is prudent to make clear that it is not the intent of this study to conflate the concepts of being a first-generation college student and of being a low-income college student. In Engle and Tinto (2008), the authors frequently reference low income, first-generation students by defining low income as coming from a family with an annual income of fewer than $\$ 25,000$. Although there is evidence that first-generation students are more likely to come from families with lower incomes (RTI International, 2019b) the two are not necessarily the same. Thomas and Quinn (2009) citing Feinstein et al. (2004ab) denote that social class is often closely related to income level, both of which are factors in opportunities and decision-making strategies. Moreover, education level is frequently associated with income and class, but again may not always align. Nonetheless, educational attainment of a parent often impacts decision-making, which further informs the opportunities for children and subsequent development of viewpoints and perspectives. As class and educational attainment are not the same, nor are they mutually exclusive, but instead interact and inform the other (Thomas \& Quinn, 2009). In sum, however, Thomas and Quinn (2009) argue, "This in-depth analysis by Feinstein et al. strongly suggests that parental education is very important in influencing the development of young children, and their success at school" (p. 73).
} 
compared to continuing-generation students (Cataldi et al., 2008; RTI International, 2019c). In fact, nearly half of continuing-generation students were found to have completed a bachelor's degree within six-years compared to merely $20 \%$ of first-generation students (RTI International, 2019c). This disparity exists despite the fact that a few metrics show how beneficial it is for firstgeneration students to complete their college degree. Specifically, after four-years of being a post-graduate, there is essentially no difference in employment rates or salary between students based upon parent's educational achievement (Cataldi et al. 2018). Why then do first-generation students not simply persist at comparable levels to their peers to enjoy the apparent practical benefits of a college degree? The answer is neither straightforward nor absolute, but the literature largely points to a disconnect between the first-generation students and the higher education environment that creates an altogether different experience for first-generation students compared to continuing-generation students.

\section{Independence vs. Interdependence}

Foremost, there is a conflict with the label "first-generation" itself, as the term is an applied categorization of students from a specific social background that largely surfaced in the 1970s (Wildhagen, 2015). The title implies that the student has done something distinct or different from their familial origins, grouping them into a new category which credits them for making an individualized decision (Wildhagen, 2015). The first-generation student label attempts to acculturate students by showing personal ability and achievement (Wildhagen, 2015). All the while, higher education has commonly been regarded as a place for free-thought and expression that closely aligns with middle-class or higher socioeconomic status (SES) (Reay, 1998; Stephens, Fryberg et al., 2012; Stephens, Markus et al., 2012; Jury et al., 2017). The feeling of individualism may inhibit and constrain first-generation students. For example, evidence 
suggests that when first-generation students approach a task framed within the context that the college environment promotes independence, first-generation students perform poorly or experience a greater sense of stress compared to the task being promoted in an interdependent environment (Stephens, Fryberg et al., 2012; Stephens, Markus et al., 2012; Jury et al., 2017). Even simply being given a plurality of choices may be troublesome to first-generation students, who unlike their middle-class peers, have been encouraged and praised for developing personal interests (Stephens, Fryberg et al., 2012; Stephens, Markus 2014; Jury et al. 2017).

Throughout the literature, there is a conflict or tension between the individuality of the first-generation students versus membership in a constructed group (Jury, et al, 2017; Orbe, 2004; Roberts \& Rosenwald, 2001; Stephens, Townsend et al., 2012; Stephens, Fryberg et al., 2012). Students have varied views, realizations of, and identification with the label of being firstgeneration (Orbe, 2004). That identification with the group is both contextual and situational, such as type of college or campus environment (Orbe, 2004). Moreover, evidence suggests that intersectionality plays a role and is more relevant for students with marginalized, prescribed, or less dominate co-identities or backgrounds (Orbe, 2004). Additionally, first-generation students often fail to construct a sense of community or unity without other students of similar backgrounds (Orbe, 2004). It is not to say that first-generation students do not identify with other first-generation students on campus, but there is a lack of group cohesion (Orbe, 2004; Roberts \& Rosenwald, 2001). Campus student groups dedicated to minority status, gender, and sexuality are commonplace and beneficial for group alliance, comradery, identity formation, support, and validation (Roberts \& Rosenwald, 2001). Perhaps in recent years more groups related to being a first-generation students have surfaced across campus; however, less common are groups 
dedicated to students who are upwardly mobile, and this is where many first-generation students would necessarily align (Roberts \& Rosenwald, 2001).

The discussion regarding the values of independence and interdependence creates a complex paradox where promoting or acknowledging a student's status may actually serve as a disadvantage to their integration to the campus community. First, the first-generation students label tends to blend a large group of students into one categorization despite this group's diverse composition (Wildhagen, 2015). Instead, some first-generation students — especially those with an intersectional identity - actively attempt to blend into their environment as a way to mask that identity or associations, partly because class associations have left them feeling underprepared (Means \& Pyne, 2017). Inherently, colleges, particularly those with greater selectivity, explicitly place value on individual merit and achievement (Jury et al., 2017). One study showed that firstgeneration students performed lower than continuing-generation students on an assessment after being told that the college had a primary goal of being selective, but performed comparably when told its goal was providing education (Jury et al., 2015; Jury et al., 2017). Another analysis showed an association between the failed persistence of first-generation students at small private colleges, with the authors hypothesizing that this population of students may dwell on or fear that their first-generation status in that environment made them standout (Lohfink \& Paulson, 2005). Dennis et al. (2005) makes the point, after exploring Asian and Hispanic first-generation students at one college, that the students originated from and valued a collectivist culture and background. However, personal and career motivations were shown to be more closely associated with adjusting to their new college environment (Dennis et al., 2005). In other words, collectivism or group adhesion was valued, but being able to leverage individuality in the higher education environment allowed the students to integrate more effectively. 
The conflict of individuality versus group association is implicitly and explicitly interwoven throughout the literature. For example, a large national survey found degree attainment among low-income first-generation students similar to degree attainment among highincome first-generation students potentially showing class or group valuations at play (McCarron \& Inkelas, 2006). High-income first-generation students may have questioned why a degree was necessary if their parents were able to succeed and be financially successful without it (McCarron \& Inkelas, 2006). However, another study found an association with an increase in family income and persistence among first-generation students between the first and second years of college (Lohfink \& Paulson, 2005). Although the studies may seem at odds, there are seemingly two factors at play, the first being social motivations related to group association (McCarron \& Inkelas, 2006) and the practical aspects of needing financial capital. The latter study specifically studied student persistence among enrolled students that would necessarily need to continue funding their education (Lohfink \& Paulson, 2005). Reay (1998) makes a valuable argument that among the middle-class students studied, higher education was seen as almost an obligatory next-step, more a matter of "where" or "what" as opposed to "if", being more interested in finding the right fit, again showing individuality at play (Reay, 1998). This contrasts with working-class students who denoted more uncertainty including finances and the proximity of school to home (Reay, 1998).

\section{The College Track: Gatekeepers and Barriers}

The aforementioned literature presents a disconnect between first-generation students and the higher education environment. In general, there are expectations for what students' value, how they will participate, or how they will integrate with the college environment. These generalities put some traditionally disadvantaged student groups at risk for failed integration due 
to a misalignment of expectations with reality by both the student and the institution. Central are two common threads that weave throughout the literature that are too entangled to discuss without at least keeping the other in peripheral consideration. The first theme is that firstgeneration students interact with myriad individuals beginning before and continuing through higher education. These individuals may serve as facilitators, barriers, or sometimes ambiguously as both. Interactions with these individuals serve as conditioning factors for the student, shaping and molding the student's habitus. The second theme is that first-generation students engage many aspects of the college's academic and social environment differently than peers, and these differences can, at times, be further delineated by other factors like race and gender. This engagement has implications for their ability to integrate and take advantage of resources on campus that are reasonably expected by the college to be beneficial for the general student body.

Well before entering college, students face differing expectations and guidance regarding higher education. Some first-generation students give credit to high school counselors and teachers as guiding them to college, which in turn provide some level of access to both cultural or social capital (Gardner \& Holley, 2011; Gibbons et al., 2019; Luzeckyj et al., 2011). For students in general, lower ratios of students to counselors as well as increased interactions with guidance counselors have shown to result in an increased number of colleges to which students submit an application (Bryan et al., 2011). Dumais \& Ward (2010) use the example of seeking guidance when crafting a college essay as "an indicator of learning how to convince key gatekeepers to grant access" (p. 255). Establishing and promoting relationships within the precollege education system may then actually help the student feel more comfortable with the college environment (Dumais \& Ward, 2010). For example, if a student feels comfortable and 
had a positive outcome with asking a counselor or teacher for help with applying to college, they may also feel comfortable seeking guidance in college (Dumais \& Ward, 2010). As argued above, first-generation students may more readily see educators and counselors as keystones to their educational aspiration and success, but if parents promote interactions, such as they themselves advocating for their student and interacting with the educational environment, then students may also then feel comfortable doing the same (Dumais \& Ward, 2010). In short, students may credit educators for being lynchpins, but it is possible that it is parents in some situations who promote that positive engagement.

Aschbacher et al. (2010), who examined the motivation of high school students to study in the science, engineering, or medical fields, opined that differing SES backgrounds experienced dissimilar "microclimates." For example, higher SES students had greater access to actual scientists or professionals and their parents more often had the means, understanding, and connections to support their educational interests (Aschbacher et al., 2010). Lower-SES students - except Asian students, who felt more encouraged-denoted numerous disparities, particularly in the school system, such as ambivalent educators, frequent substitute teachers, and counselors that portrayed science as too challenging (Aschbacher et al., 2010). Male AfricanAmerican and Latino first-generation students felt particularly pushed toward structured co- or extracurricular activities (e.g., sports) to make sure they "avoided trouble", but female students, particularly Latinas, felt pushed to fulfill familial cultural gender roles (Aschbacher et al., 2010, p. 578). In a study of seventh grade students, prospective first-generation students already denoted lower self-efficacy regarding attending college and recognized more barriers that included, among other things, family issues, limited educational role models, and limited reliable resources for college attendance (Gibbons \& Borders, 2011). 
In sum, the literature shows that first-generation students have different opportunities for accessing experiences that provide educational benefit for college. An undertone is that firstgeneration students are predisposed to certain paths based on externally conceived expectations for their future. Much of this may have to do with how much parents are guiding or promoting interactions with the educational system. As the literature shows, greater formal interactions, such as with educators and counselors is a means to access capital. However, even then, the literature shows that formal interactions may steer some students to particular activities based upon their gender or race.

\section{Self-Efficacy of first-generation students}

The literature is mixed when attempting to explicate how first-generation students perceive their abilities upon entering college. One study argues that first-generation students may enter with a high-level of self-efficacy, and do not look for support from those, such as parents, who are not perceived as having a reliable viewpoint (Westbrook \& Scott, 2012). Atherton (2014) contends that although first-generation students enter higher education with typically lower metrics such as grade point average (GPA) and test scores, they do not necessarily recognize their deficiencies or that college is going to be academically different. This failed acknowledgement is perhaps because family members provide a limited means of social and cultural capital (Atherton, 2014). In an additional study among a sample of first-year students at a private liberal arts college, first-generation students reported significantly lower self-efficacy scores than continuing-generation students, but their level of self-efficacy was not necessarily a factor in their GPA performance (Ramos-Sanchez \& Nichols, 2007). A variable to consider, however, is the type of institution in which the studies were conducted. Selectivity or at least its perception, may elicit poorer performance among first-generation students (Jury et al., 2015; 
Jury et al., 2017), which could be related why students at a private liberal arts college had lower self-efficacy.

Alternatively, neither Vuong et al. (2010) nor White et al. (2017) ascertained a difference of academic self-efficacy based upon generation status. Vuong et al.'s (2010) study did highlight an association between self-efficacy, performance, and persistence in sophomore students in the California State system (Vuong et al., 2010), while White et al. (2017) found association between perceived financial stress and academic self-efficacy. Therefore, some evidence does show that first-generation students may overestimate their abilities, simply based upon misunderstanding of what to expect academically and intellectually in college. However, not all studies show that first-generation students had different levels of academic self-efficacy compared to continuing-generation students peers. The literature has not concluded if part of the difference may relate to the specific backgrounds from which samples of first-generation students may originate or if perhaps student's perceive self-efficacy differently based upon the environment of their college campus (e.g., selective versus non-selective admission).

\section{College Preparation}

Self-efficacy aside, first-generation students are academically less prepared because of less access to advanced educational opportunities in high school, already putting them behind their peers (Engle \& Tinto, 2008). This topic was already broached above with Ashbacher et al.'s (2009) notion of exposure to "microclimates." Some characteristics of first-generation students in high school include being less likely to take advanced placement courses, less likely to have an academically focused curriculum as well as less likely to have advanced math courses (Cataldi et al., 2018). ${ }^{7}$ Advanced math courses are of particular importance as they are associated

\footnotetext{
7 “An academically focused curriculum includes a minimum of four credits of English; one credit of mathematics higher than algebra II and any two other credits in math; one science credit higher than general
} 
with four-year college attendance (Engle \& Tinto, 2008 citing Choy, 2001 \& Horn \& Nunes, 2000). Although first-generation students are as likely to take a math course in their first-year as continuing-generation students, they are less likely to take an advanced math course (RTI International, 2019c). Both low-income and first-generation students are also typically more likely to take remedial courses in college (Cataldi et al., 2018). In a large survey of student characteristics at six American research universities, the authors found first-generation students more likely to self-identify as having weak English and math skills compared to middle- and upper-class students, which were characterized as obstacles to their academic success (Stebleton $\&$ Soria, 2012). Other studies have denoted first-generation students felt they had lower computer skills (Jenkins et al., 2009) and reading skills (Byrd \& Macdonald, 2005).

\section{Academic Integration}

Despite a multitude of challenges at play, for even the most prepared first-generation students, academic integration is a problem. Central to the challenge is a recasting of a "learning identity," where a student moves from greater levels of certainty to uncertainty (Christie et al., 2008). ${ }^{8}$ When students lack or miss "embedded knowledge" it can have impacts on their confidence to perform (Christie et al., 2008). Means \& Pyne (2017) found that first-generation students, even those with high personal achievement (e.g., valedictorian), felt that they did not fit in academically on their campus. "This lack of a sense of academic belonging was not about students' academic self-efficacy: instead, it hinged on their limited access to a rigorous, collegepreparatory curriculum when compared to their more affluent peers” (Means \& Pyne, 2017, p. 916-917). A major challenge to students' ability to academically integrate is that they may not

biology and any two other credits in science; one credit of social studies in U.S. or world history and any two other credits in social studies; and two credits in a single foreign language" (Cataldi et al, 2018, p. 5). ${ }^{8}$ Christie et al. (2008) specifically examined non-traditional students at an elite Scottish University and not specifically first-generation students. 
recognize how, or the need, to engage the system in which resources or experiences exist to make transition easier (Soria \& Stebleton, 2012; O’Shea et al., 2017; Woosley \& Shepler, 2011). Grayson (2011) argues that first-generation students in college may rely more on personal characteristics or skills that brought success in high school (Grayson, 2011). The author found that Canadian first-generation students were both less engaged with academic and social activities on campus, and made fewer friends compared to peers, but the only significant predictor of first-generation students' GPA in their first-year was their high school GPA. Conversely, new friends, academic involvement, class experiences and GPA all significantly predicted the GPA of continuing-generation students (Grayson, 2011).

Evidence shows that common activities on college campuses are either ill-understood or under-engaged by first-generation students (Banks-Santilli, 2004; Collier \& Morgan, 2008; Kim \& Sax, 2011; O’Shea, et al., 2017; Soria \& Steblteon, 2012). This argument is further accentuated by Woosley and Shepler (2011), who by using Tinto's (1993) longitudinal attrition model, argue that continuing-generation students and first-generation students essentially need similar services and support to engage and persist in the college environment. Student services or academic experiences provided on the college campus are available to support first-generation students persistence, but students have not been primed or conditioned to recognize their value or necessity (Woosley \& Shepler, 2011). First-generation students in the literature have shown to be more likely to be dissatisfied with faculty advising (Kim \& Sax, 2007), did not understand faculty office hours (Collier \& Morgan, 2008), struggled to grasp or recognize the value or use of course syllabi (Banks-Santilli, 2014; Collier \& Morgan, 2008), did not recognize important financial aid concepts (Gibbons et al., 2019; O’Shea, 2007), struggled with scheduling processes (O’Shea, 2007; O'Shea, 2015a), and were challenged with even more mundane campus concepts 
like learning a public transportation system (Banks-Santilli, 2014). Not knowing the environment both causes the student to miss these essential components, but also necessitates a time investment to learn them (King et al., 2019), a challenge for a group already overextended (Byrd et al., 2005; Mehta et al., 2011).

Luzueckyj et al. (2017) specifically contributed to the literature an analysis of metaphors that first-generation students utilize to explicate their transition into and through higher education. Some of the metaphors used by first-generation students include, needing to "learn the new language of the 'foreign land"' (O'Shea et al., 2017, p. 199), "fish out water", "lone wolf", being on a journey, and being pushed off a diving board and needing to learn to swim—all of which illicit some level of unfamiliarity and necessitates adaptation (Luzueckyj et al., 2017). In a study of Australian female first-generation students, a number of students characterized their earliest time in college as surviving, which was more directed as getting through the financial, personal, and time commitments necessary (O'Shea, 2015a). Another study found that firstgeneration students worried that perhaps going to college was "too bold" and devised exit strategies or ways and times to step-out of the environment if needed (O'Shea et al., 2017, p. 207).

Interestingly enough, even having a parent with marginal college experience can dramatically alter how a student interacts and integrates with the environment. Researchers found that students entering college from a family where there was just some college experience by a parent(s) that did not graduate, had more substantial cognitive and psychological development during the first-year of college than compared to first-generation students from families with no experience (Padgett et al., 2012). Similarly, in an Australian study, firstgeneration students had significantly lower reported academic application (e.g., motivation, 
consistency in effort, seeking out educators, desire to do well, etc.) compared to students with at least one parent with a college degree (McInnis \& James, 1995). The point is that being successful is less about a parent having a college degree, but more about being exposed to individuals and opportunities that either know or may help promote the rules of the game (Padgett et al., 2012). Students have a role to learn and play to facilitate advancement and success (Collier \& Morgan, 2008; Demetriou et al., 2017; Groves \& O’Shea, 2019). Firstgeneration students tend to be slow to in engage those roles perhaps because, "they know less about the importance of engagement and how to become engaged" (Pike \& Kuh, 2005, p. 209).

This point is further supported by looking at the trends of first-generation students in graduate programs. This population is important because as noted, first-generation graduates entering the workforce are as employable as peers (Cataldi et al., 2018) so it may stand to reason that by graduating with an undergraduate degree that the first-generation students is now also prepared for graduate education. In fact, Choy (2001) contends that first-generation students were as likely to enroll in a master's degree program as continuing-generation students. However, they were less likely to continue on to a doctoral degree program or a professional degree program (Choy, 2001; Engle \& Tinto, 2008). Moreover, once in graduate school, firstgeneration students were less likely to complete their degree (Franklin \& Slate, 2012). For those first-generation students who did finish, they were less likely to continue on for a second graduate degree (Franklin \& Slate, 2012). At least one study contends that it is possible to identify both attrition risk-factors for both continuing-generation students and first-generation students graduate students, but first-generation students are significantly more at risk for attrition with the following five factors: delayed enrollment, part-time enrollment, full-time employment, financial independence, and the number of dependent children (Seay et al., 2008). Evidence 
shows that postgraduate first-generation students are employable at percentages and salaries comparable to their peers (Cataldi et al., 2018), but there is a disunity and continued challenges for first-generation students, even in graduate school.

The literature surrounding academic integration shows that first-generation students struggle to assimilate on campus. Even though a multitude of support services exist on college campuses, first-generation students lack the intrinsic knowledge to access those services. They may also not understand their value or have individuals in their lives who can promote their utilization. first-generation students may feel themselves on the outside looking in and alone in their journey. Particularly germane to this study, the literature shows that these struggles do not abate just by simply graduating with an undergraduate degree, but also carry over into graduate education.

\section{Faculty Interaction}

Generally, faculty interactions with students are among the most effectives methods to aid student academic integration on campus, maintain retention, and encourage degree completion (Kuh et al. 2010; Tinto, 1993). It is also among the most challenging and intimidating for first-generation students (Padgett et al., 2012) who arrive with skewed ideas of how they will interact with faculty (Hicks, 2003; Padgett et al., 2012). In a survey of a precollege preparation program, first-generation students and continuing-generation students were found to have notably different expectations and understandings of the role of faculty instructors with first-generation students indicating they believed instructors would tell them how they were doing in a course, be concerned with their performance, teach study skills, and provide feedback (Hicks, 2003). In some of the aforementioned categories, a post-program survey instead showed more similar responses between the continuing-generation students and first-generation students 
potentially indicating that even tangential or early college interactions may begin to calibrate expectations of faculty's role (Hicks, 2003).

After starting college, academic interactions for first-generation students are markedly different from their peers. In one study, first-generation students in small classes (60 students) were more likely than continuing-generation students to interact outside of the classroom with faculty and TAs about class, but the opposite was true in larger classes (150 students) (Beattie \& Thiele, 2016). The authors argue that this pattern may be a result of including "TA" in the survey question and continuing-generation students being comfortable with engaging TAs (Beattie \& Thiele, 2016). Interactions, all around, were particularly less common for African American students and Hispanic students (Beattie \& Thiele, 2016), who are more likely to be firstgeneration students (RTI International, 2019b). These findings are important because many firstgeneration students are likely to enter large universities and start in large lecture halls (Beattie \& Thiele, 2016). These interactions, or lack thereof, can be detrimental for students who arrive on campus with limited social capital and then fail to harness associated benefit from formal interactions with faculty such as future career networking (Beattie \& Thiele, 2016).

In general, first-generation students have been found to be less likely to ask faculty members for help (Jenkins et al., 2009) while instead recognizing peers as sources of guidance or help (Dennis et al., 2005; Roberts \& Rosenwald, 2001; Soria \& Stebleton, 2012), less likely to interact via email or face-to-face conversations with faculty (Kim \& Sax, 2007) including in class (Kim \& Sax, 2007; Soria \& Stebleton, 2012), and more likely to voice frustration or confusion in understanding what faculty want from assignments (Collier \& Morgan, 2008). Moreover, one study showed first-generation students less likely to have paid research positions with faculty — but equally as likely to conduct research voluntarily or for academic credit (Kim \& 
Sax, 2012). The research opportunities predicted positive impacts (i.e., aspiration, communication, critical thinking and GPA) for both first-generation students and continuinggeneration students, but only found a significant impact on sense of belonging for continuinggeneration students (Kim \& Sax, 2012). General faculty interaction was found to predict similar outcomes including integration for both groups, but while continuing-generation students saw an improvement in GPA, this same relationship did not exist for first-generation students (Kim \& Sax, 2012).

One study found that first-generation students thought faculty interaction to be central to their success, but felt uncomfortable reaching out to faculty and fretted about wasting their time, which was ameliorated when faculty reached out first (Means \& Pyne, 2017). Another denoted that first-generation students were at times intimidated by faculty because of their perceived use of discipline jargon (Collier \& Morgan, 2008). Regarding academic advising, in a large study of student trends in a subset of the California University system, first-generation students were less satisfied than continuing-generation students (Kim \& Sax, 2012).

Academic advising is particularly important as advising is a means for students to gain access to the formal academic aspects of the college-i.e., cultural capital (Swecker et al., 2013). To emphasize this point, in a regression analysis of academic advisor interaction by firstgeneration students, for every meeting with an academic advisor, the odds of retaining the student until the next fall increased by 13\% (Swecker et al., 2013). Along the same lines, a study of low-income first-generation students found that faculty who specifically leveraged teaching strategies that fostered positive faculty interactions, and among other things, connected with students about personal challenge and closely followed their work led to students feeling greater connections with faculty and classes (Schademan \& Thompson, 2015). "These cultural agents 
[faculty] created a space in their classrooms that promoted a sense of connection and belonging to the larger campus community" (Schademan \& Thompson, 2015, p. 18).

Although positive impacts of faculty engagement are certainly true, it is not quite as straightforward as may be expected. The argument to be made is not that first-generation students do or do not receive positive impacts from faculty interaction. Instead, first-generation students seek out and value interactions differently than peers, and certain interactions merit different gains than others. Padgett et al. (2012) recognized that there is a theoretical basis for the argument that student-faculty interaction can benefit persistence, integration, and cognitive development. However, in a multi-institution quantitative study, the authors found that firstgeneration students who had positive faculty interactions actually had lower scores on both the Need for Cognition scale and the Ryff Scale of Psychological Well-Being (Padgett et al., 2012). ${ }^{9}$ The authors make the point that these students, with perhaps less social capital, may see the experiences as intimidating and not necessarily appreciate their value (Padgett et al., 2012). Instead, first-generation students saw greater benefit than continuing-generation students from interactions with peers and from academically challenging experiences (Padgett et al., 2012). In a study of student persistence from first to second year, academic integration on campus was found to associate with persistence for first-generation students but not continuinggeneration students (Lohfink \& Paulson, 2005). ${ }^{10}$ Central to this finding is that many of the items in the academic integration index utilized relies on faculty-student interaction (Lohfink \&

\footnotetext{
${ }^{9}$ The authors claim that, "A high score for cognition denotes a greater need to seek, engage, enjoy, and reflect back on cognitive activities. In contrast, those with low need scores are more likely to rely on others, cognitive heuristics, or social comparison processes to make sense or evaluate their world" (Padgett et al., 2012, p. 263). "The Ryff Scales of Psychological Well-Being tend to have positive associations with measures of happiness and satisfaction and negative associations with depression" (Padgett et al., 2012, p. 263).

${ }^{10}$ The authors denoted the academic integration index as, "An NCES index based on four BPS items indicating how frequently the student had social contact with faculty, met with an academic advisor, talked with faculty about academic matters outside of class, or participated in study groups during the 1995-96 academic year" (Lohfink \& Paulson, 2005, p. 425.).
} 
Paulson, 2005). The authors (Lohfink \& Paulson, 2005) further reference previous literature (Rendon, 1993; Terenzini et al, 1994) that argues nontraditional student's need for validation or recognition of their abilities in-light of the current environment. Pascarella et al. (2004) argue, "First-generation students perhaps benefit more from their academic experiences than other students because these experiences act in a compensatory manner and thus contribute comparatively greater incremental increases in first-generation students' stock of cultural capital" (p. 280). Therefore, the literature shows that first-generation students struggle to engage faculty and often fail to recognize its value, this is despite of that fact that first-generation students commonly see large gains in academic integration when they do have positive faculty experiences. Faculty interaction is a central aspect of first-generation students engaging the college environment. All students have been shown to benefit from faculty engagement, but it is of utmost importance for first-generation students as this is a formal means to engage cultural capital of the college campus.

\section{First-Generation Social Interaction}

As already noted, first-generation students are more likely to turn to peers for help or guidance on campus (Dennis et al., 2005; Soria \& Stebleton, 2012), despite the fact that they may also struggle to relate to continuing-generation students peers (London, 1989). Moreover, in a national sample of students, first-generation students were also found to have lower-levels of aspiration (highest level degree sought) and more likely to live off campus, in conjunction with less academic and social engagement, fewer gains in intellectual and learning development, and less-positive perceptions of collegiate environment compared to continuing-generation students (Pike \& Kuh, 2005). Pike and Kuh (2005) argue aspiration and living off campus impacts the ability to engage with the college environment and subsequently reap the benefits of those 
interactions (Pike \& Kuh, 2005). To further complicate the suggestion that first-generation students may not understand the benefit of on-campus social interaction, one study found that overall satisfaction with social-life was a factor in persistence between the first- and secondyears for both continuing-generation students and first-generation students (Lohfink \& Paulson, 2005). However, social-life may have different connotations for the two populations. Firstgeneration students were more likely to persist if they chose the college so they could live at home or if English was not the first-language in the home (Lohfink \& Paulson, 2005). These two findings would indicate the importance of family within the student's social network (Lohfink \& Paulson, 2005).

Interactions with peers, particularly those who are not first-generation students, are important because they serve as means to accessing dominate cultural and social capital on campus (King et al., 2019). Demetriou et al. (2017) explains in their findings that successful first-generation students at one institution attended class and other academic-centered functions, which became portals to new relationships and opportunities (Demetriou et al, 2017). For example, going to class may lead to participating in a study group, interacting with peers, finding commonalities, joining other organizations, and so forth. (Demetriou et al., 2017). This type of activity relates back to that of learning the role of how to be a college student (Collier \& Morgan, 2008; Demetriou et al., 2017; Groves \& O’Shea, 2019). In sum, much of the literature shows the value of social interactions as they can become means to further integration with the overall environment. Moreover, engaging peers can lead to participation in other events or opportunities, which can further enhance investment and commitment to success. This type of engagement, though, can already be a challenge for first-generation students because they may be living off campus or have personal commitments, which puts them at a disadvantage. 


\section{Time Commitment, Employment, \& Wellbeing}

Part of the challenges for first-generation students is time to participate in on-campus events as they are likely to work more hours (Drozd, 2008; Grayson, 2011; Mehta et al., 2011; Pascarella et al., 2004; RTI International, 2019a) or live off-campus (Pascarelle et al., 2004; Pike \& Kuh, 2008). Evidence suggests that first-generation students may have less financial capital from their parents to pay for college (Mehta et al., 2011), and overall, more reliance on grants, loans, and scholarships, which may be challenging to navigate (Gibbons et al., 2019; Martinez et al., 2009; Mehta et al., 2011). In a quantitative analysis at a single university, researchers found a significant difference in the amount parents contributed to tuition, with first-generation students receiving much less financial support from parents but more from loans and grants (Mehta et al., 2011). Additionally, another single university qualitative study found a similar reliance on both grants and loans, but also scholarships (Gibbons et al., 2019). In fact, the authors characterize money as both a barrier and a means of support for first-generation students (Gibbons et al., 2019). Despite finding resources initially, students struggled to manage their money and explicated not understanding basic concepts surrounding resources, including the need to reapply for aid annually to keep the funds (Gibbons et al., 2019). This trend, although limited to one study, is in light of the fact that nearly two-thirds of first-year first-generation students in a national sample denoted that they engaged financial aid services (RTI International, 2019b).

Regarding work, a large national survey found that about $66 \%$ of first-generation students, compared to $61 \%$ of continuing-generation students, held a paying job, an internship or work-study, but also worked more hours per week and were less likely to work on campus (RTI International, 2019a). This, of course, would leave less time for social and academic interaction on campus (Pascarella et al., 2004). However, Martinez et al. (2009) found that for first- 
generation students, part-time work did not predict student attrition, but full-time work did, delineating a difference in how much a student works, but not if they worked. Moreover, by citing Tinto (1993), the authors intimate that there may be some benefit for students participating in paid work as it does foster some socialization and integration (Martinez et al., 2009). Of course, it seems reasonable that more funding directly to first-generation students (Pascarella, 2004), or perhaps ways to deter full-time work, may serve to support first-generation students (Martinez et al, 2009). This finding is further supported by Martinez et al. (2009) who found not having a scholarship, as well as having high levels of loans, were factors in predicting attrition rates. However, students in an institutional needs-based scholarship program were able to integrate with peers and felt supported by the college (Martinez et al., 2009).

How the transition to college effects the overall wellbeing and coping ability of firstgeneration students is largely unresolved in the literature. A quantitative study from a single, large institution found that risk factors for attrition in their student body included, for example, psychological distress, heavy alcohol usage, and drug usage; however, first-generation students were not found to be significantly more at risk for any of the aforementioned factors (Martinez et al., 2009). These findings are in contrast with others who found in various samples of firstgeneration students to have greater instances of depression, stress (Afeli et al., 2009; Mehta, et al., 2011), or both (Jenkins et al., 2013; Stebleton \& Soria, 2012). Jenkins et al. (2013) found first-generation students reported feeling less support from peers and family, more depressive symptoms, more posttraumatic stress markers (perhaps attributed to SES factors), and lower lifesatisfaction. In part, these findings may associate with academic acculturation, or an attempt to acclimate to an environment that is unfamiliar (Jenkins et al., 2013). These issues were more 
pronounced in women, to which may be attributed to balancing social-gender norms-including feelings of needing to be a support system for others such as male partners (Jenkins et al., 2013).

Some of the literature has pointed to the fact that first-generation students feel overwhelmed or that they have too much to balance between academics, family, and work (Afeli et al., 2018; Byrd, \& MacDonald, 2005; Gibbons et al., 2019; Jenkins et al., 2009; Mehta et al., 2011; Stebleton \& Soria, 2012). To illustrate how this may impact a first-generation student's potential, in a look at pharmacy students, continuing-generation students were more likely to denote missing class because of co-curriculars, being out the preceding night, and the time of the class (e.g., too early), while first-generation students cited, for example, stress, employment, and family activities (Afeli, et al., 2008). A major challenge to dealing with bouts of both depression and stress is the proposition that first-generation students have fewer outlets to offload these feelings as they have limitations in both peer and family resources (Barry et al., 2009; Jenkins et al., 2013). In other words, there is a vicious cycle at play as limitations of social and financial capital predispose first-generation students to certain circumstances, their ability to deal with those challenges is not easily ameliorated because of limited social capital (Jenkins et al., 2013).

There is some indication in the literature that first-generation students actually cope with stress differently than peers (Mehta, et al., 2011). Mehta et al. (2011) found first-generation students would attempt to cope mentally or take time off work to deal with stress, although it was not clear if this was to focus attention on other academic areas. Notably, though, first-generation students were less likely to deal with their stress through social outlets like participating in night life or going to bars, which would otherwise provide evidence of coping with stress through alcohol usage (Mehta et al., 2011). The literature has not explored if other types of alcohol usage, such as binge drinking, are common among first-generation students (Mehta et al., 2011). In a 
small study at a single institution, researchers found that first-generation students had lower levels of emotional intelligence, but higher levels of resilience (Alvarado et al., 2017). One posited reason for the mismatch is that resilience was developed from life experiences before college, which is a theme found elsewhere in the literature (Alvarado et al., 2017; Luzeckyj et al., 2017; O’Shea \& Delahunty, 2019) and explored below. Interestingly, particularly high achieving first-generation students, unlike continuing-generation students, have been found to move to academic performance-avoidance goals, or "goals [that] focus on avoiding the demonstration of inferior competence" (Jury et al., 2015, p. 2). Jury et al. (2015) cite a myriad of negative outcomes associated with performance-avoidance, but generally associated activities and pressures that can impact overall personal wellbeing. A number of reasons for this behavior could include, among other things, concerns about being stereotyped, struggles with social or class identity, and self-doubt (Jury et al., 2015).

In sum, the literature has pointed to the fact that first-generation students struggle with balance school and personal commitments, including work to support themselves. Some of the stress in dealing with multiple responsibilities and roles can have deleterious effects, including anxiety and depression. First-generation students may also deal with concerns of failure or worry that they may be perceived as inadequate. Although there is a dearth of literature regarding how first-generation students actually deal with these feelings, some evidence shows that they do not attempt to cope socially, such as by engaging alcohol in a group setting. Instead, they may internalize and attempt to power through. This area of the literature needs further expansion as a great deal of research has gone in to identifying where students may struggle to engage the college environment, but not so much regarding how they personally deal with the stresses of that engagement, which seem to be overwhelming for some. 


\section{The Role of Family}

To this point, much has alluded to the dynamics at play regarding changing family relationships. As first-generation students struggle in the new collegiate environment, the also struggle with a unique experience of family separation. A number of scholars have pinpointed a disconnect that surfaces between the first-generation students and the family (Covarrubias \& Fryberg, 2015; Hsiao, 1992; London, 1989; McInnis \& James, 1995; Reay, 1998; Robert \& Rosenwald, 2001; Thomas \& Quinn, 2009). That disconnect is bidirectional where the family senses a disconnect with their child — the student—and visa-versa. Meanwhile, the student struggles to manage established relationships (i.e., forms of social capital) and new relationships. London (1989), provided an early contribution to better understanding the phenomenon denoting that as first-generation students drift more from the family there is chance for reflection and consideration of the roles and dynamics of the family. The student becomes more independent and begins an inconstant transition away that the family does not identify with, all the while the student may not be able to relate experiences to middle class or continuing-generation students peers (London, 1989). Students are left feeling a "biographical or social dislocation" triggered by relationship renegotiations (London, 1989, p. 144).

The literature is mixed in describing how students ultimately begin to renegotiate their own personal and family identity (London, 1989; Robert \& Rosenwald, 2001). For firstgeneration students who seek an advanced or graduate degree, a feeling of familial separation may actually intensify. In a study of $\mathrm{PhD}$ students, some first-generation students denoted pride from their families; however, others felt they needed to alter their actions or the way they spoke around family members for fear of being perceived as arrogant or conceited (Gardner \& Holley, 2011). This was particularly troubling for those students, who in an academic sense, struggled 
with feeling like a phony or an imposter at school leaving them sensing both a detachment from family and their professional lives (Gardner \& Holley, 2011). These findings may be of particular importance for this research because attending medical school is also a step beyond completing a four-year degree.

Many first-generation students actually experience "family achievement guilt" that is akin to "survivors' guilt" in that familial and cultural interdependence and esteem for "important others" is held in high regard (Covarrubias \& Fryberg, 2015, p. 421). Higher education is a foray into independence that affords opportunity and personal accomplishment unavailable to their most central and formative social circle (Covarrubia \& Fryberg, 2015). However, some feelings of guilt may actually be ameliorated when first-generation students find ways to help or aid family members, which further supports the notion of a conflict between balancing independence versus social interdependence (Covarrubia \& Fryberg, 2015). Seemingly counter to the argument that first-generation students may experience higher levels of guilt, though, Votruba (2009) found no difference in presence of interpersonal guilt among first-generation students versus continuing-generation students. Feelings of interpersonal guilt were associated with academic integration, and furthermore, academic integration was found to be lower among students who left campus more frequently (Votruba, 2009). Integration did not appear to be impacted by frequency of communication with friends and family at home (Votruba, 2009). These findings are interesting because although guilt may not have been necessarily more prevalent in firstgeneration students, guilt impacted integration, and integration was impacted by time off of campus.

With these aforementioned dynamics in mind, the literature is quite mixed on the role of families as barriers, a support system, or both for first-generation students (Banks-Santilli, 2014; 
Barry et al., 2009; Delong, 2003; Gofen, 2009; Gibbons \& Borders, 2010; Gibbons et al., 2019;

Hicks, 2006; Jenkins et al., 2013; McCarron and Inkelas, 2006; McInnis \& James, 1995; O’Shea, 2007; O’Shea, 2016; Roberts \& Rosenwald, 2001; Stieha, 2010; Thomas \& Quinn, 2009; Westbrook \& Scott, 2012). One of the frequent findings replete through the literature is that parents may at times provide emotional and personal support, though this is often limited in scope and does not extend into more academic or practical guidance (Barry et al., 2009; Gibbons et al., 2019; O’Shea, 2007; O'Shea, 2016; O’Shea et al., 2017; Roberts \& Rosenwald, 2001; Westbrook \& Scoot, 2012). That is to say that parents may lend their support and encouragement, but the students often felt that their parents failed to truly understand their plight. Moreover, although first-generation students could lean on their parents as a basic support system or for practical assistance (e.g., childcare) (O’Shea, 2007), they did not necessarily see their parents as able to provide either academic guidance or insight into transition to higher education (McInnis \& James, 1995).

The literature is divided on whether students can effectively relay their challenges to parents and the ultimate relationship of these disclosures to their personal wellbeing. Some argue that a parent need not fully understand to be a confidant for the student (McInnis \& James, 1995). However, if first-generation students feel closed off from traditional support systems (i.e., family and peers from home) they have fewer outlets to relay their experiences and challenges, which can have negative impacts like higher stress levels (Barry et al., 2009). Interestingly, though, Dennis et al. (2005) found that among ethnic minority first-generation students, the students thought peers, as opposed to family, were more appropriate for support related to “college related problems" (p. 229). The authors make the point that students still looked to parents for emotional support, but specifically, perceptions of limited peer-support correlated 
with outcomes like a lower GPA (Dennis et al., 2005). This finding falls in line with the idea that first-generation students are looking to peers for academic or environment specific support (Dennis et al., 2005; Roberts \& Rosenwald, 2001; Soria \& Stebleton, 2012) and that parents may not be legitimate sources of information (Westbrook \& Scott, 2012).

Arguably, part of the disconnect with family, however, relates to their perceived utility of a college education. Bradley (2017, p. 41) makes the case that students from more middle-class backgrounds make an obligatory transition to college as way to adulthood and their time there is buttressed by "structure of expectations" from the family (Luzeckyj et al., 2011). On the other hand, first-generation families may be more likely to question if the student's studies are practical and related to the "real world" (Robert \& Rosenwald, 2001, p. 100). The literature shows the first-generation parents recognize college as a means to an end, or a practical way to find a more secure vocation or career that ultimately brings some security to the family_ an acknowledgment of social mobility from a college education (Delong, 2003; Lehman, 2009; Thomas \& Quinn, 2009).

Delong (2003) who engaged parents of first-generation students, finds that these parents look at their children's decisions through the cultural and social lenses from which they are currently situated. The parents placed high value on local social networks, family proximity, compliance with family norms, and preconceptions of the passage to adulthood (e.g., marriage and family) (Delong, 2003). Nonetheless, parents may still support their children even if they do not understand their decisions (Delong, 2003), but also recognize limits like proximity and perceived appropriate career fields to achieve those ends (Thomas \& Quinn, 2009). They do not see college experience as unimportant; but instead, there is a need for cohesion in the family unit that should not be upset (Thomas \& Quinn, 2009). Again, this returns to the concept and conflict 
of the individuality of the educational experience versus group constructs and cohesion, as the individual student effectively breaks from the group for personal gain (Thomas \& Quinn, 2009).

Other literature has more explicitly argued families as essential components to firstgeneration students success. In one study, first-generation students denoted perceptions of greater support from their parents (Hicks, 2006). Moreover, first-generation parents may be more involved with providing support by believing that engaging the educational system and seeking out information is essential for the student's success (Hicks, 2006). This contribution meshes well with the notion presented above that there is a perceived need to convince gatekeepers for access (Dumais \& Ward, 2010), and perhaps parents are seeking those gatekeepers for their children. Additionally, there is a normalization process that takes place for the family when a first-generation students enrolls in college (O'Shea, 2015b), contributing to "demystifying the university experience and encouraging access for others" (King et al., 2019, p. 108), and in some cases inspiring other family members to seek higher education (O’Shea, 2015b; Wainwright \& Watts, 2019). First-generation student's interactions with the college environment creates "bidirectional flows" of understanding between both the home and the college (O'Shea, 2015b, p. 153).

Gofen (2009) presents an oft-cited study of Israeli first-generation students and argues that parents must leverage aspects of family capital, such as instilling values, beliefs, and priorities that promote education as valuable (Gofen, 2009). Essentially, the argument made is that to break the intergenerational disparities related to accessing education, family as a microcosm, must begin introducing their children to capital that is not necessarily social—related to association or connections - nor cultural, related to valuations-but instead, family capital (Gofen, 2009). Family capital is defined as, "The ensemble of means, strategies, and resources 
embodied in the family's way of life that influences the future of their children. Family capital is implicitly and explicitly reflected through behavior, emotional processes, and core values" (Gofen, 2009, p. 119). Gofen (2009) specifically found traits of first-generation students to include family solidarity, ambition, and aspiration and connect to the family's views of the student and education, in general. "Simultaneously, parents put their children first among the family's priorities and expect them to succeed in their studies. The parents in this study deeply believed in their children's abilities and intensely expressed their belief' (Gofen, 2009, p. 116). The article is an important contrast to others because it formally implicates family capital as an association with educational success. Interestingly, though, it almost makes it seem that for firstgeneration students there needs to be a formal recognition to recast ideals toward education, as where the notion of education being valuable is implied among continuing-generation students.

McCarron and Inkelas (2006) support the notion that specific parental and family involvement are major determining factors in the development of education aspirations among not just first-generation students, but all students. ${ }^{11}$ In fact, the authors found in a large national survey that parental involvement was the greatest predictor for continuing-generation students aspiration, and the second highest predictor for first-generation students aspiration - the first being perception of good grades (McCarron \& Inkelas, 2006). Aspiration is a particularly important consideration as it has been linked to persistence among both continuing-generation students and first-generation students (Lohfink \& Paulson, 2005), but has been found to be typically lower among first-generation students (Lohfink \& Paulson, 2005; Pike \& Kuh, 2005). Patfield et al. (2020) opined that families of first-generation students commonly leverage aspiration through three types of family dynamics. Those include projected familial habitus,

\footnotetext{
${ }^{11}$ McCarron and Inkelas (2016) denote parental involvement to include frequency of helping with homework as well as frequency of conversations concerning ACT/SAT preparation, current classes, and going to college.
} 
which includes discussions of post-secondary education that present opportunity for the future, meritocratic familial habitus, which advocates students to work-hard and have personal responsibility in their education and achievement, and supportive familial habitus, which encourages the educational decisions and ambitions of the student (Patfield et al., 2020). In many ways, these three mechanisms are not too distant from notions put forth from Gofen (2009) that there is a formal investment in family capital.

These types of family habitus described by Patfield et al. (2020) also help relate back to the questions surrounding if a first-generation family can provide appropriate support for the student. Perhaps the parent cannot typically provide an intricate understanding of the college transition, the higher education system, or academic aid, but they most certainly can provide support that fosters some level of aspirational benefit for the first-generation students by simply promoting the experience. O'Shea et al. (2017) ultimately makes the point that among firstgeneration students they studied, there was not an inherent lack of aspiration or motivation. "However, these strongly motivated potential students also often feel like imposters who regard university study as a high-stakes game where they do not know the rules" (O'Shea et al., 2017, p. 207).

In sum, a great deal of literature has looked at the role of families and parents supporting first-generation students. However, first-generation students are often caught between two worlds, one of which they are familiar with and the other they are trying to access. Family may not always be seen as reliable sources of information to engage that latter world, but nonetheless, they are important for providing general support, even if they cannot provide practical information. Moreover, parents may only be able to provide guidance from their current cultural or social viewpoint, which can add to the mixed messages and signaling perceived by first- 
generation students. An interesting point in the literature, though, is that first-generation students may actually leverage certain aspects of capital from their backgrounds to succeed in the new environment, which is expounded upon in the next subsection.

\section{Strengths and Class Movement}

Although a great deal of the literature surrounding first-generation students focuses on challenges in the higher education environment, there is mounting evidence that first-generation students bring specific qualities and sources of capital, which they leverage for both aspiration and inspiration (Luzeckyj et al., 2011; O’Shea, 2016; O’Shea, 2019). In other words, firstgeneration students may redirect capital germane to working-class backgrounds to engage middle-class capital (Lehmann, 2009). Life experiences, including having dependents (Engle \& Tinto, 2008; RTI International, 2019b), generally being older (RTI International, 2019b), likelihood of military service (RTI International, 2019b), and more work experience (Engle \& Tinto, 2008; RTI International, 2019a) all contribute to a plurality of beneficial qualities of firstgeneration students found in the literature. Lehman (2009) coined this as first-generation students and working-class students recognizing the benefit of "moral advantages" (p. 639). For example, the literature has found first-generation students pull upon higher levels of appreciation (non-entitlement) (Tate et al., 2015), maturity (Lehmann, 2009; O’Shea, 2016), life experiences (Byrd \& Macdonald, 2005; O’Shea, 2016), time-management skills (Byrd \& MacDonald, 2005), goal focus (Byrd \& MacDonald, 2005; Tate et al., 2015), self-advocacy (Byrd, 2005; Tate et al., 2015), work-ethic (Lehmann, 2009; O’Shea \& Delahunty, 2019), and grit, resilience, or determination (Alvarado et al., 2017; Luzeckyj et al., 2017; O’Shea \& Delahunty, 2019; Tate et al., 2015) to journey through the higher education experience. 
These findings seem rational. For example, two studies found that the student or their parents were challenged with "dead-end job(s)" that helped train their focus on certain goals (Tate et al., 2015, p. 296) or led to their own motivation (Byrd \& MacDonald, 2005, para. 26). O'Shea (2016) specifically found that first-generation students gained aspiration from recognizing their social status or feeling stuck in the system (i.e., receiving government benefits), which engendered a level of resistance capital. Many of the skills or qualities denoted could be attributed to any number of experiences. For example, juggling many tasks could contribute to other skills like better time-management skills (Byrd \& MacDonald, 2015). However, some findings are more curious than others, including self-advocacy, especially when the literature discusses uncomfortably with interacting with formal college systems (e.g., faculty advising) (Byrd \& MacDonald, 2005; Tate et al., 2015; Kim \& Sax, 2007). Perhaps part of the disconnect is that the two studies (Byrd \& MacDonald, 2005; Tate et al., 2015) engaged first-generation students perceptions, including the viewpoint of not having resources for guidance and needing to find a way forward on their own. This idea would relate back to discussions of feeling as a lone wolf or needing to learn to swim (Luzueckyj et al., 2017).

Interestingly, first-generation students may bring certain skill sets or capital to the table that they foster from a more working-class background in an effort to engage with a middle-class environment (Lehman, 2009; Luzeckyj et al., 2011; O’Shea \& Delahunty, 2019). Even though students may engage the skills sets that they know, it does not mean that they necessarily recognize how or what skills or experiences to leverage to their benefit (O'Shea \& Delahunty, 2019). O’Shea \& Delahunty (2019) found, “...instead the learners in our study had to complete additional and often invisible work to apply their existing capitals to their university activities" (p. 165). It was not until after the fact that students recognized what did or did not work and what 
skills they utilized (O'Shea \& Delahunty, 2019). Again, this points to the concept of learning to play a role in the college environment (Collier \& Morgan, 2008; Demetriou et al., 2017; Groves \& O'Shea, 2019), to which first-generation students may need to tryout or engage less obvious skills (O’Shea \& Delahunty, 2019). To this point, O’Shea and Delahunty (2019) argue that higher education often fails to help first-generation students and underrepresented students engage these skills or backgrounds as assets for the academic journey.

First-generation students have also been shown in the literature to begin to recognize their own personal changes. As first-generation students move through higher education and persist, their perceptions of the environment do change and they essentially begin adapting or conforming to its norms and expectations. Part of this, Groves and O’Shea (2019) denotes a movement from the periphery to interacting with more central or legitimate networks (i.e., building cultural and social capital) or learning to play a role (Collier \& Morgan, 2008;

Demetriou et al., 2017; Groves \& O’Shea, 2019). Moreover, there is some evidence (Dumais \& Ward, 2010) that after students are admitted and begin processing through higher education, utilizing capital from their habitus becomes less of a factor as they are likely to begin understanding and adopting new tools suitable to the environment. King et al. (2019) posit the first-generation students walk away with access to new opportunities, diverse perspectives, the opportunity to be role models for others, and among other things, a greater commitment to learning, connections to peers (i.e., social capital), a changed worldview, greater self-efficacy, and self-confidence.

Difference among both male and female first-generation students have also been noted. In their day-to-day lives, O'Shea (2015a) found that Australian female first-generation students recognized a greater sense of personal-control by attending college. Many noted greater self- 
confidence and in some respects a change in their domestic relationships with a spouse or partner, some of which included changing roles or expectations of family members (2015a). Other indicated a change with their children intimating that going to college would help redirect the educational prospects of her family as well as the social implications (e.g., breaking a cycle of poverty) (O’Shea, 2015a).

In a separate study of male Australian first-generation students, men were not found to experience a masculinity crisis by going to college (O'Shea et al, 2017). Fathers in the study leaned on the idea of needing to gain credentials for employment as they aged, which harkens to some traditional concepts of being the family's breadwinner (O'Shea et al., 2017). The older fathers in the study saw college as a way to pursue dreams or personal interests (O'Shea et al., 2017). Similarly, the coined "self-starters" were presumably not fathers, but were older than the traditional students and were seeking higher education for either personal interest and motivations or for career reasons (O’Shea et al., 2017). Men who had a developed male identity did not as actively struggle with an identity crisis; however, the coined "sons" of the studymore traditional aged male college student_ - did intimate greater levels of stress in their new experience (O'Shea et al., 2017). They noted that Australian anti-intellectualism and masculinity norms add to feelings of separation or misunderstanding with family (O'Shea et al., 2017).

Hinz (2016), who specifically examined how working-class first-generation students experience class mobility reengaged Hurst's $(2007,2010)$ loyalist and renegades typology to include two additional types, mobile loyalists and converts. The crux of the reframing is that class-movement is not one-size fits all change, but instead, may result in a rejection, weariness, acceptance (including a dual acceptance), of either originating class or new class location (Hinz, 2016). For example, "Loyalists and Renegades have a negative opinion of the class with which 
they do not identify, so much so that Loyalists fear becoming middle-class and Renegades fear not becoming middle-class. Mobile Loyalists and Converts are more flexible. They can view their class identity through multiple lenses and allow their class identities to be more complex..." (Hinz, 2016, p. 297).

In sum, the point of this subsection is that first-generation students most certainly arrive with a multitude of class or group specific skills that they utilize to adapt to and traverse the higher education experiences. This has been well documented in the literature. However, the dislocations experienced during higher education do not dissipate. As students craft a new identity through college, they leave attempting to ascertain where they stand socially, which to some, means a rejection or acceptance of working class or middle-class identities. For others, it may mean ambiguity. Moreover, these experiences and outcomes are often mediated by other intersectional identities like gender.

\section{Conclusions}

The literature is replete with studies that target a multitude of aspects of the firstgeneration student experience. Those studies include aspiration and preparation, the experience on campus, differences between first-generation students racial and social groups, the role of gatekeepers, peers, and family, the strengths and weaknesses commonly denoted among firstgeneration students, and how the first-generation student changes through the higher education experiences. The literature has also highlighted tensions with concepts like the term "firstgeneration" and the student's background as well as how higher education's solution for firstgeneration student's success as providing resources for the student to recognize and use to adapt to the environment. That is to say, the literature shows that higher education looks upon the student as in some way unprepared or deficient instead of reflecting on how higher education 
fails to adapt for the first-generation students. As a result, first-generation students rely on many of their own skills more germane to their backgrounds to, almost paradoxically, exist and be successful in the new environment.

Subtly and expressively throughout the literature, there is clear conflict between the concept of individuality promoted in higher education versus the feelings of class or group association that first-generation students are likely more comfortable ascribing to. This conflict involves both trying to establish bearings in the college environment, which expects a certain type of implicit knowledge and behavior expected of the individual versus reconciling an assigned group association attributed to the student's class and family. There is a sense of being caught in two worlds, unable to fully commit to either, further hindering integration. The literature presents the idea that first-generation students may see college as a possible or crucial next step, but they lack the knowhow and resources to fully and effectively engage the environment. Moreover, by further engaging the environment, they experience a greater conflict with family and their original social networks. To be clear, it is not that first-generation students consciously recognize their deficiencies or that they intentionally choose to avoid certain resources, but rather, the student does not even know that they are missing resources or that they may be unprepared.

\section{Part 2: Rural and Appalachian Students}

The second section of this literature review moves to include an analysis of both the rural and Appalachian student experience. ${ }^{12}$ This review begins with the decision that rural teenagers

\footnotetext{
12 As Manly et al. (2020) note, there are a number of definitions of "rural" used in the literature. Moreover, those definitions have tended to transition and change over time, even when originating from the same source. Because of that, as a caveat, the authors warn that it is necessary to keep in mind that differences, even subtly, may exist in the literature as it relates to the definition of what is or is not considered rural (Manly et al., 2020). It would be too protractive for this literature review to recount every utilized definition or conceptualization of rurality; therefore, it is necessary to recognize the possibility that differences likely exist between studies.
} 
must make regarding staying in or leaving their community. Moreover, if they leave, will they return? Does going to college mean they can readily access other options in their community, or does it begin the process of being pulled away for external opportunities? For those who do stay, what is the motivation to do so-family, a place attachment, or local career paths? Donaldson (1986) argues that young rural adults struggle with "the attempt to reconcile attachments to community and past with a desire-or economic need - to be a part of the modern American mainstream" (p. 122). The stayers versus leavers literature is a backdrop for other questions, including, why do so few rural students, particularly Appalachian students, not pursue higher education? To be clear, there is a distinction between the decision to stay or leave and the decision to pursue higher education. One could leave for education or other pursuits, and still return. However, this review seeks to better understand how education factors into that decisionmaking process. From there, how do rural and Appalachian students either seek out or reject higher education? How does local culture and social circles drive that decision-making? Finally, this review concludes with a look at the college experience for rural and Appalachian students including struggles with integration

\section{Stayers versus Leavers}

Leavers are those who step away from their community for other opportunities with no guarantee of returning with new skills or education, contributing to a rural "brain drain," (Carr \& Kefalas, 2001). Communities with limited resources often invest in the future of their children, but do not necessarily reap direct benefit of college educated returners (Carr \& Kefalas, 2001). Carr and Kefalas (2001) who created a typology of leavers versus stayers, characterized two types of leavers with the first being "seekers," those who left but had less means for college so they often chose the military, while "achievers" were those that left for college likely not to 
return (Carr \& Kefalas, 2001). Students who finish post-secondary education and return are exceedingly valuable for the community as they bring back educational capital (Gibbs, 1995). Those returners are needed as role models for the next generation of potential college-goers (Gibbs, 1995). Oftentimes, policy-makers focus on wooing leavers back to the local economy instead of providing educational resources to those who stay in the hopes highly educated or trained individuals with a community attachment will return (Carr and Kefalas, 2001). The act of leaving, though, is not an easy one as many rural youths face an internal conflict, depression, emptiness, anger, or hopelessness, while males often sense the greatest discord (Hektner, 1995; Elder, et al., 1996).

Leaving, though, is not necessarily a lifetime commitment. "Returners" or "boomerangs" - often women — are those that come home after a post-secondary opportunity, including military service or completing a two- or four-year degree (Carr \& Kefalas, 2001). Gibbs (1995), has argued that home is a "counterweight" to outside opportunities, particularly after achieving post-secondary education (p. 43). For those who do not return, the stayers are actually vital to maintaining communities and connections; without stayers, leavers who have much less pull back as community and social ties would weaken (Stockdale \& Haartsen, 2018). The act of staying has been regarded by some scholars as a conscious decision and not necessarily immobility, instead capitalizing on already built social capital (Barcus \& Brunn, 2009; Morse \& Mudgett, 2018; Stockdale \& Haartsen, 2018). By staying, family and community ties are already established, which generates shared and identifiable customs, norms, and values (Carr \& Kefalas, 2001; Hektner, 1995; Stockdale \& Haartsen, 2018). This notion that staying is a conscious decision draws a contrast with an argument put forth by Beasley (2011), who noted during a study of a WV county that, "It is important to remember that there is a difference 
between individuals who choose to return or stay and those who feel as though they have no choice but stay" ( $\mathrm{p}$ 231). The notion is that leaving means succeeding but staying is failing (Beasley, 2011; Foster \& Main, 2016).

\section{An Economic Decision}

The literature shows that deciding to stay in or leave the community involves economic calculations (Bjarnason \& Thorlindsson, 2006; Corbett, 2005; Cox et al., 2014; Johnson et al, 2002). A factor in that decision relates to the likelihood that rural communities in some way associate with specific industries that limit local economic diversity (Lichter \& Brown, 2011). Corbett (2005) found that mobility was largely dictated by educational attainment, with the greater the education obtained the further the movement. However, for boys, education was regarded as less necessary because the local economy was an accessible male-dominated fishery (Corbett, 2005). Women, instead, were more mobile inside the region, either seeking credentials through educational opportunities due to limited labor or seeking employment in the service industry (Corbett, 2005). However, young adults, regardless of gender, who received postsecondary education but stayed in the community actually had limited job opportunities and were unable to access attained educational capital (Corbett, 2005). Corbett (2005) demonstrates the challenge at hand for many youth — there is a local and undiversified job market geared toward a male-dominant industry. College, as argued below, is not necessarily valued as a viable option and for those who do attend college, their prospects become more limited in the local economy.

\section{Collectivism and Social Networks}

Much of the literature regarding the rural student's path to college hinges on the effects and makeup of the social network of the students. Rural students benefit from tight, but dense, social circles including a strong association with kinship confined to the community, a 
phenomenon known as social closure (Bjarnason \& Thorlindsson, 2006; Byun, Meece, \& Irvin, 2012; Coleman, 1988, 1995; Singh \& Dika, 2003). Bjarnason \& Thorlindsson (2006), argue that Coleman $(1988,1995)$ :

[Conceptualizes] social closure as a specific form of social capital that harnesses the human capital of parents to the benefit of their children on the individual level, and a collective good that benefits all children on the community level. He maintains the resources of the community as a whole constitute a form of collective human capital that will only affect adolescent well-being when activated through a process of social closure. (p. 298)

Social closure is a necessary concept to keep in mind, as there is evidence of this closure at play in both rural and Appalachian communities as it relates to the trajectory and aspiration of adolescents.

For Appalachia, there is a documented feeling of collectivism in much of the rural population (Gore et al., 2011; Leon \& Hoffman, 2016; Wilson \& Gore, 2009). Community and kinship ties are a valuable aspect of Appalachian culture resultant of generations of sequestration from prevailing American culture (Tang \& Russ, 2007). Beasley (2011) found that WV youth recognized the value of extended family networks harkening the mantra of taking a "village" to raise a child (p. 214). For Appalachians, the literature has found that many who desire to live close to home are more satisfied with their relationships within the community and the family as opposed to being particularly attached to the place (Barcus \& Brunn, 2009).

Rural social structures' impact on college-going decision making for rural youth has been closely examined in the literature. Rural student access to community resources has correlated with a higher likelihood of completing a college degree (Byun, Meece, \& Irvin, 2012). 
Community resources, for example, include social circles with greater likelihood that parents know their children's friends and their families, participation with a church community, and strong kinship ties (Byun, Meece, \& Irvin, 2012). This is confirmed with a study that examined social circles in rural southwestern Virginia (Singh \& Dika, 2003). Researchers found perceptions of academic and emotional support were most closely related to positive student academic and psychosocial outcomes, and social networks with greater numbers of nonfamily members with higher education levels predicted higher education aspirations (Singh \& Dika, 2003). However, this general support was fairly superficial, such as encouragement to do well and set a good example for others, but not necessarily practical or insightful (Sigh \& Dika, 2003). Additionally, any benefit that is received from accessing community resources is often offset by the propensity for these rural areas and youth to face low-SES hardships, which undercut gains from community encouragement and support (Byun, Meece, \& Irvin, 2012).

It bears noting that Appalachian students are also reliant on the roles and viewpoints of the extended community in making the college decision (Powell, 2008). Brown et al. (2009) found the greater overall educational attainment in Appalachian rural communities corresponded with higher educational goals among youth—especially among young men—regardless of other factors like the educational level of parents or poverty. However, actual educational attainment among these students seemed to be less related to the educational level of the community, but instead more closely tied to parent's education and financial status (Brown et al., 2009). The implication is that the community, at large, does impact the aspirations of Appalachian youth, but parental and family influences remain a central factor for development and realization of those aspirations (Brown et al., 2009). 
A tightknit rural community can also have negative side-effects (Arnold, 2017). For example, young adults may already have preconceived identities crafted by others due to family affiliation (Beasley, 2011). Additionally, Appalachian students have complained that social activities in their hometown were often only related to either the local high school or their church (Arnold, 2017). Others have explicitly found that strong associations with community institutions have little bearing on if a student desires to stay in the community (Johnson et al., 2002).

In sum, the literature shows that rural students can benefit from tightknit social circles, because it increases the likelihood of meaningful interactions that can promote post-secondary education — such as, interacting with college educated community members. Overall, students may sense support that can stoke aspiration, but the encouragement does not always go further. This literature shows a conflicting tension where rural social settings may have qualities conducive to educational encouragement and support, but actual attainment is stymied and more closely related to family resources. Rural communities, particularly in Appalachia, have a greater propensity for low-SES families; therefore, any support from the community is largely undone by more pragmatic reasons such as needing to think about the more immediate future. There is also the notion that overly tightknit social circles can actually lend to some youth wanting to escape community constructed identities.

\section{Educators, Resistance, and Value in Rural Communities}

How rural student's perceive academic support from educators and local educational systems is mixed throughout the literature. In some Appalachian studies, educators and the school systems are perceived as apathetic about the student's post-secondary path and provide an obligatory role of graduating students or raising test scores and GPAs to meet funding standards 
(Arnold, 2017; Morton et al., 2018; Powell, 2008). Lack of expectation results in demotivation among students (Powell, 2008). There is also the notion that schools essentially groom the already high performers to leave by providing them with better educational resources and experiences (Carr \& Kefalas, 2001; Chenoweth \& Galliher, 2004; Powell, 2008). At the same time, low achievers, or those who struggle academically, receive additional classroom support (Powell, 2008). The connotation is that middle of the road students find themselves stuck, not recognized as a high achiever, but not weak enough to receive exclusive aid. Beyond instruction, rural students have intimated that they did not see their teachers or their school counselors as having adequate or sufficient information regarding college (Morton et al., 2018) and that college is portrayed as having insurmountable challenges (Friesen \& Purc-Stephenson, 2016).

Others have rebutted the notion that the highest achievers are groomed with the best resources to leave. Instead, smaller academic functions and extra-curricular programming at rural schools lends to greater opportunity for students to participate, which leads to greater comfortability (Petrin et al., 2014). The literature also points to the fact that some students see teachers as motivating to go to college and that certain individual teachers were lynchpins to their success (Beasley, 2011; Griffin et al., 2011; Hlinka, 2017; Powell, 2008; Welch, 2013). In fact, rural students' level of educational aspirations and high school persistence has shown to associate with their teacher's expectations (Byun, Irvin, Meece et al., 2012; Roscigno \& Crowley, 2001). Demi et al. (2010) make a particularly compelling argument that when rural students perceive their school's climate as positive, it tends to create a number of effects including increased student self-efficacy, educational aspirations, and grades, which can assist or nudge a rural student to continue their education (Chenoweth \& Galliher, 2004). ${ }^{13}$ These findings

\footnotetext{
${ }^{13}$ Demi et al. (2010) denote that school climate was assessed by asking students, for example, perceptions of relationships with educators, fairness, safety, if they felt cared for, etc.
} 
do not refute those above intimating that students did not see their educators as providing adequate academic preparation or support for college, but taken together, it presents that notion that aspiration among rural students is related to the educator's expectation. Moreover, some evidence has shown that rural students, as they mature, look more to teachers and guidance counselors for direction regarding college as opposed to just family (Griffin et al., 2011).

The dynamic between educators and rural students does suffer from a cultural divide of sorts. The literature has documented a level of resistance to school that surfaces among Appalachian working-class students (Hendrickson, 2012). Part of that resistance arises when the student is exposed to concepts at school perceived as irrelevant or conflicting with family views favoring local community and values, including diverse cultures and viewpoints (Hendrickson, 2012). These ideas, coupled with the promotion of going to college, clashes with other routes like seeking a skilled vocation (Hendrickson, 2012). Although high school influencers may promote rural students seeking practical post-secondary degrees that show a track to future employment, some parents still resist the pathway (Tieken, 2016). The mixed-messaging from both sides further complicates the decision-making process for students (Tieken, 2016).

Rural schools, in general, occupy a unique space in small communities often becoming a focal or rallying point - a sense of pride (Beasley, 2011). However, rural communities, with limited economic diversity or with lower SES populations, also underinvest into the educational system (Roscigno \& Crowley, 2001; Roscigno et al., 2006). Rural and inner-city school districts appear to actually have similar investment in the local educational resources (Roscigno et al., 2006). However, after accounting for available resources at both the family and school level (e.g., family income, education, size, etc. \& school racial makeup, expenses per student, free lunches, etc.), inner-city school investment is more closely aligned with suburban schools 
(Roscigno et al., 2006). The implication is that rural schools have lower investment in local education than even low-income inner-city districts, which can signal what is or is not valued by that community (Roscigno et al., 2001; Roscigno et al., 2006). AP courses, for example, are an important indicator for a localities' value of education as they only directly benefit college-going students and are typically less available in rural communities (Roscigno \& Crowley, 2001). Similarly, rural high school students see fewer opportunities to participate in advanced math courses compared to suburban peers, likely due to lower volumes of students prepared to actually take those courses (Irvin et al., 2017).

One study that documented perceptions of adolescent students in a college preparatory program at an Appalachian low-income school found participants liked math and sciences and knew that going to college would likely mean needing advanced coursework, but they also lamented their math abilities (Meehan et al., 2001). This finding corresponds to another study denoting that Appalachian college students struggled and stressed about their math courses (Beasley, 2011). Lack of preparation can be a major detriment to Appalachian college students as remediation or development courses can create additional financial burdens, elongate time degree, and for some, feel as though they are confirming stereotypes of being unintelligent (Powell, 2008). The point is that lack of resources and access at a community level can have years of lasting effects on students who wish to pursue higher education. Rural schools providing fewer resources for educational opportunities melds with an argument put forth by Elam (2002) concerning Appalachia. Essentially, there is a long history of poverty in the region resulting from a myriad of reasons including marginalization, isolation, underfunding, reliance on certain industries, etc. and views of education as an individual pursuit have not always resonated as a 
public need (Elam, 2002). These factors have festered into a cultural insularity of sorts implicating an undervaluing of education (Elam, 2002).

To briefly summarize, the literature shows that when it comes to investment, rural and Appalachian communities lag behind more suburban and even urban areas. Students may have mixed perceptions on how they value or perceive their educators in preparing them both academically and practically for college. However, the fact remains that messaging received at school is at odds with what students hear at home. Therefore, there is a tension between what students may be hearing and how the message is perceived given that parents may be presenting differing values. This issue further is complicated by communities underinvesting in educational opportunities.

\section{Self-Efficacy Among Rural Students \& Preparation for College}

The literature shows that for rural students, academic self-efficacy relates specifically to engagement to the educational process as well as feelings of social support. For example, rural students with higher self-efficacy have been found to positively predict both career expectations and educational aspiration (Meece et al., 2014; Wettersten et al., 2005). Students who value their educational experience and recognize it as essential for their future plans also point to generally higher educational aspirations (Meece et al., 2014). Wettersten et al. (2005) argues that rural students who feel support from peers, family, and the school regarding their education and high academic self-efficacy also intimate high engagement with their education and greater future career expectations. For Appalachian students, Roush (2013) found that students credited supportive peers for high school success, partly because of extensive periods of time with the same social circles. These findings are not necessarily surprising considering the valuations that rural and Appalachian students put on their social circles. 
Major barriers for Appalachian students, particularly those who are first-generation students, are actually the inability to recognize college going challenges, which results from knowing or understanding so little about higher education (Gibbons et al., 2020). Students are lax and generally lack reliable information - a "practical disconnect" of sorts — seeing the process through cultural terms and failing to implicate the nuances of preparation (Gibbons et al., 2020, p. 176). For example, Appalachians students lack urgency and rely on notions that things will work out if they simply work hard while missing obstacles like finding a way to finance their education (Gibbons et al., 2020). Part of the challenge may actually relate to the concept that in the Appalachian culture, there is already a strong reliance on family for guidance and support, but with college, they may not get reliable advice or instead get lackadaisical or ambivalent direction (Ali \& Saunders, 2006). Appalachian students, though, who have shown greater levels of vocational/educational self-efficacy — such as, the confidence or ability to seek out information germane to post-secondary education — had greater college and career aspiration (Ali \& Saunders, 2006 \& 2008). Similarly, Tang \& Russ (2007) have argued that Appalachians could greatly benefit from methods that enhance vocational self-efficacy because, among other reasons, it could foster other valuable traits including agency and persistence.

In Appalachia, students with Science, Technology, Engineering, Mathematics or Medicine/Healthcare aspirations are more likely to be continuing-generation students, have shown higher self-efficacy regarding going to college as well as greater "college process preparedness" (Carrico \& Matusovich, 2016; Rosecrance et al., 2019). This is important for this study as it seeks to better understand the experience of Appalachian medical students who must navigate a multitude of obstacles and expectations (e.g., prerequisites and requirements) to matriculate into a medical degree program. Some evidence show that Appalachian students 
actually show particular motivation to serve in healthcare related fields, due to the fact many students and young adults in the region become caretakers for family members and begin to see it as a calling that motivates their studies (Bradbury \& Mather, 2009).

For rural students able to participate in college preparatory programs, curriculums, or college outreach programs, there is plenty of evidence of positive impacts on student aspirations - both educational and occupational (Arnold, 2017; Bryan \& Simmons, 2009; Carrico \& Matusovich; Chenoweth \& Galliher, 2004; Meece et al., 2013; Powell, 2008). Moreover, students with high college preparedness cared less about local labor prospects and were not particularly compelled to stay in the community (Carrico \& Matusovich, 2016). Similarly, other research has found that when Appalachian students met with admissions officers, they more readily recognized the positive financial effects that a college degree could have for their future (Powell, 2008). Taken together, this would imply that students more educated about college also understand long-term implications of a college degree that includes more career opportunities not tied to just local options.

Rural and Appalachian students with higher academic and college going self-efficacy are better positioned for going to college than their peers. However, challenges remain, including failing to foresee some of the more pragmatic issues on the horizon, such as paying for college. Many are able to garner support from peers and family, but again, these are not necessarily reliable sources of information. This can also lead to an underestimation of what is actually needed to attend college. Evidence shows, though, that college preparatory opportunities and interfacing with actual authorities in the matter can be beneficial. However, it is unclear from the literature just how widespread preparatory programming is available in Appalachian communities given the argument above concerning family weariness of education in general. 


\section{Family Influences}

The literature shows that the influences and roles of the family in a student deciding on a post-secondary path is multifaceted and interacts with other characteristics of rural life. For example, Johnson et al. (2002) argues that perception of local opportunity can drive other outlooks, including that young rural adults who recognized tougher local job prospects did not see staying close to family as that important. This is at odds with other literature that denotes maintaining a connection with family is central to deciding to stay or leave a rural community (Morse \& Mudgett, 2018). This feeling of family cohesion may relate to financial concern and the need to stick together (Elder et al., 1996; Hektner, 1995). As rural youth mature, the family context is constant and central, with that constant becoming a basis for many other aspects of their life (Elder et al., 1996). Decisions related to the local economic situation and finances come later when approaching adulthood, but kinship and collectivity are palpable earlier (Elder et al., 1996). This is best summarized by Lichter \& Brown (2011) who note, "For the least educated, however, spatial differentials in wage rates are often small, which anchors low-skilled rural residents to place, while elevating the relative importance of other factors (such as family and friends) in making decisions to stay or leave rural areas" (p. 572). To briefly summarize, the literature shows that although family is vital and some assuredly argue it remains at the forefront of the decision-making process, the implication of job prospects lingers over those relationships.

How a student perceives their relationship to parents is not exactly a great determinate of the desire to go to college or not — as having a strong attachment with parents does not necessarily steer a rural student to or from higher education (Demi et al., 2010). While on the other hand, when rural adolescents respect or want to be more like their parents, they feel a strong attachment to wanting to live nearer to them (Johnson et al., 2002). This nuance shows 
that identifying with parents may or may not indicate if that student will follow a certain path, but it does influence wanting to stay close to the family in the long-term. The insinuation being that leaving for college could be seen as temporary, and not necessarily indicative of where the student will reside.

As a predictor of if students will go to college, evidence shows that parent or family education level impacts rural students' postsecondary aspirations (Brown et al., 2009; Carrico and Matusovich, 2016; Demi et al., 2010; Dyk \& Wilson, 1999; Powell, 2008; Rosecrance et al., 2019). Parental attitudes about education also consistently surface as an important point for the student's development of greater aspirations and educational attainment (Ali \& Saunders, 2006; Beasley, 2011; Byun, Meece, Irvin et al., 2012; Demi et al., 2010; Dyk \& Wilson, 1999; Hlinka, 2017; Hunley, 2015; Meece et al., 2013; Meece et al., 2014; Schmitt-Wilson, 2013; Wettersten et al., 2005). Family attainment and attitudes are important, but community ties also remain a factor. For example, the literature shows that in conjunction to parent's educational level and attitudes, parental employment can also help guide a rural student to college as more desirable jobs in the community may put the student in connection with "valued others" — such as college educated supervisors or colleagues delineating an example of influential positive social networks (Carrico \& Matusovich, 2016, p. 272).

Despite the positive benefits regarding educational attitudes, the literature shows that rural parents are less involved with both the educational process and have lower educational expectations (Byun, Meece, Irvin, 2012). In a look at participants in GEAR-UP, ${ }^{14}$ Appalachian students noted their parents did help them with schoolwork, particularly mothers, but parents

\footnotetext{
${ }^{14}$ GEAR-UP or Gaining Early Awareness and Readiness for Undergraduate Programs is a federally funded college preparatory program that aims to provide, among other things, services and support to prepare low-income middle school and high school kids as well as their parents, for the college experience.
} 
were under-engaged, as evidenced by failing to see value in parent-teacher conferences (Meehan et al., 2001). This cohort of students, while participating in a college development program, had high educational aspirations and most wanted to go to college, but had limited knowledge about college itself (Meehan et al., 2011). For example, students indicated one barrier to college as cost, but beyond a basic understanding, students and parents did not expect scholarships as likely and did not know about grants and work-study (Meehan et al., 2001). Students saw their parents as keepers of pertinent information, despite no evidence that parents interacted with the school to find out about requirements for graduation or college (Meehan et al., 2001). Mothers did, however, intimate greater interest than fathers in attending free workshops to learn about related topics (Meehan et al., 2001). The point is that rural and Appalachian students commonly look to parents for information about going to college (Ali \& Saunders, 2006; Meehan et al., 2001) and parents may be generally supportive, but that does not mean they are prepared or know how to help students (Gibbons et al., 2020; Hand \& Payne, 2008; Morton et al., 2018). Rural parents do not always understand their role (Darling, 1999). One study of Appalachian first-generation students used the example of the typical college orientation, which was ill-understood as a necessity for the parent since they themselves were not going to college and it required them to take time away from work (Darling, 1999). Rural WV parents misunderstood concepts like admission, saw college as for the exceptionally smart or wealthy, associated college with TV portrayals (i.e., party atmosphere), feared outsiders could take advantage of their child, and worried what the community may think if their child did not succeed—emphasizing a narrative of risk (Snyder, 2007).

It is important to remember that as students in rural settings participate in robust but closed social structures, in Appalachia particularly, family and kinship values are central (Ali \& 
Saunders, 2006). Therefore, even if parents are positive influencers, extended family networks may be more ambivalent, discouraging, or unconvinced that college is an appropriate route (Beasley, 2011; Hand \& Payne, 2008; Powell, 2008). Some have pointed out that rural families tend to be larger, which could put a strain on already tight family resources (Roscigno \& Crowley, 2001; Roscigno et al., 2006). Others, though, have refuted that, arguing larger families do not impact the odds of rural students going to college (Byun, Meece, Irvin et al., 2012). In fact, rural students actually look to siblings for college-going guidance (Griffin, Hutchins, \& Meece, 2011), and among Appalachian first-generation students it becomes a point of pride for the older student (Bush, 2015). This is, in effect, an example of rural students leveraging navigational capital—leaning on those who have successfully managed the educational process (Bejarano \& Valverde, 2012).

Families of rural students have strong influences on their aspiration and ability to attend college. However, as already argued, the literature shows that parents may not always know their role, not always have reliable information, or worry about the risks involved. These trends show an under-engagement with the process among rural parents, which is to the detriment of their child's aspiration and attainment. Additionally, kinship ties and input remain important and can also influence aspirations and attainment, though not always negatively. In fact, rural students recognize those with high educational attainment as role models in the process.

\section{Trajectory by Gender}

The Appalachian region has a long-established cultural patriarchy and these notions often underpin the guidance and direction each gender receives regarding their future career options (Helton \& Keller, 2010; Hunley, 2015; Tang \& Russ, 2007). The literature is conflicting on between how mothers and fathers impact their child's aspirations and paths. The higher the 
educational attainment of parents — particularly mothers - has shown to associate with greater educational attainment of rural females (Blackwell and McLaughlin, 1999). While Appalachian literature has opined that a fathers' educational aspirations for their child can have a major impact on the child's educational trajectory (Ali \& Saunders, 2006), particularly for sons (Chenoweth \& Galliher, 2004). Some have posited that since mothers are less present in the local workforce, they may be regarded as less influential on career path decisions (Chenoweth and Galliher, 2004). Though the connotation that mothers with less engagement in the workforce may cause them to be less influential in the student's career trajectory is not universally accepted in the literature. For example, some Appalachian literature has noted mothers as primarily helping with homework, more likely to have met the child's teacher (Meehan et al., 2001), and more commonly identified as the most important individual in the child's social network (Singh \& Dika, 2003). Moreover, in Appalachian low-income families, the mother's educational attainment as well as educational and occupational expectations were shown to be important for a student's own academic performance as well as their educational expectations in high school and beyond (Dyk \& Wilson, 1999). Others, still yet, have suggested a substantial amount of variance by family in the type of support Appalachian first-generation students receive from either the mother or father (Wood, 2012).

As there are nuances in the literature regarding influence of mothers and fathers, there are also notions that young men and women are pulled by differing social forces. Foremost, rural women are typically more likely to enroll in college and perceive they are expected by parents to achieve more education (Agger et al., 2018; Meece et al., 2014). Even when Appalachian women do not always hear they should attend college, they sense that they should do well in school endorsing educational achievement (Welch, 2013). Aspiration and perceptions of expectations 
may relate to the need to consider educational options in lieu of immediate career opportunities (Meece et al., 2014; Agger et al., 2018). In fact, Blackwell \& McLaughlin (1999) found that higher levels of unemployment in a rural community associated with more time females spent pursuing education. Because rural women already recognize fewer career opportunities, they also see greater competition for work and in turn begin to look toward doing well in school to create some level of distinction (Dyk \& Wilson, 1999; Elder et al., 1996). In a related finding, Appalachian women were more likely to attend college based upon self-efficacy as well as exposure to college concentrated curriculums (Chenoweth and Galliher, 2004). This finding would correspond to the idea that female students saw a need to distinguish themselves and thus set higher expectations based upon on perceptions of their own ability.

There are also preconceived ideas of what types of college education is appropriate based upon gender. For example, Appalachian women are more often expected by families to become a nurse or a teacher — which also provides opportunity to work within the local economy (Beasley, 2011; Blackwell \& McLaughlin, 1999). Even so, rural women are actually more likely than men to seek education and careers less traditional to their gender for reasons that may include modern influences, career latitude, and opportunities through college preparation programs (Meece et al., 2014). In support of this argument, high school girls in an Appalachian study were more likely to aspire to a science, technology, education, math or medicine field and had overall greater selfefficacy regarding getting to and persisting in college, even though first-generation girls pursing these fields were less interested in the content matter (Rosecrance et al., 2019).

There is also a divergence between the role of social circles for both young women and men as they mature. Young women actually see the lowest educational attainment gains from extracurricular participation — with the exception of honor societies - compared to rural men, and 
urban men and women (Blackwell \& McLaughlin, 1999). For college going guidance, rural females are less likely to go to community members, but instead go directly to recruitment material and college representatives to gather information (Griffin et al., 2011). Although women may sense a greater need to go to college, there is also evidence that rural women may be directed away from college to instead fulfill traditional gender roles such as becoming a spouse and primary caretaker for children (Donaldson, 1986; Dyk \& Wilson, 1999; Snyder, 2007). Those who are pushed or follow tracks aligned with gender norms may lead to a closing social circle and further challenges in accessing the local labor market (Dyk \& Wilson, 1999). An additional nuance is that rural females have higher levels of educational aspiration, but they also intimate greater feeling of family responsibility such as needing to live at home longer and assist in raising siblings (Agger et al, 2018; Meece et al., 2014). Rural girls who were perceived by their teachers as having positive traits such as being connected and engaged with the educational process were more likely to also have a strong rural identity and desire to stay local (Petrin et al., 2011). Therefore, female students with higher school engagement are more likely to desire staying in the community, despite the prospect that local career options are more limited. There is a disconnect between seeing educational performance and college as more pressing due to lacking local opportunities coupled with shaking off traditional gender careers, but also a need to follow traditional gender pathways that include being family caretakers that further binds the student to the area (Egan, 1993).

For men, social circles tend to expand, which also lends to more conversations and connections regarding job opportunities in the community (Dyk \& Wilson, 1999). This point is reinforced by the argument that community support for education is important for young men as they look outwardly for guidance, emphasizing the need for college educated role models 
(Blackwell \& McLaughlin, 1999). For Appalachian students in general, there is not much evidence that peers have a particularly strong influence on college planning compared to other social circle members (Wood, 2012). Young men, however, do gain more positive influence regarding college through social interaction than do young women. For example, Appalachian males were more likely than females to seek peer input on deciding if they should go to college (Chenoweth \& Galliher, 2004). Likewise, rural males were found to have higher educational attainment from involvement in high school extracurriculars, including scouting and sports, but not vocational organizations (Blackwell \& McLaughlin, 1999). Young rural men have also been found to be more responsive to support and expectations of their high school teachers (Byun, Meece, Irvin et al., 2012). Griffin et al., (2011) found rural men compared to females were particularly more apt to talk to coaches about college options, but did not notably talk to any other members in the community. This finding neither confirms nor refutes the notion that young men's social circles expand, but instead, it simply shows that college talk may be less pressing or generally uncommon in-light of local opportunities as young men mature and social circles expand. In other words, young men face a multitude of influencers as they reach adulthood. On one hand there are positive college influencers who may be teachers or coaches while on the other are community members who are gateways to local job opportunities.

The decision to seek a college education among young men is particularly related to the local economy, finding immediate work, or simply because college is irrelevant in the short-term (Bjarnason \& Thorlindsson, 2006; Chenoweth \& Galliher, 2004; Corbett, 2005; Friesen \& PurcStephenson, 2016; Snyder, 2007; Wallace \& Diekroger, 2000). One study found that two major factors associate with rural men not attending college including a feeling of rural identity and perceived local employment options - a mix between attachment and practical opportunities 
(Agger et al., 2018). Similarly, a desire to stay in the community along with family SES and parents' educational level were all factors in WV boys deciding if they wanted to go to college (Chenoweth \& Galliher, 2004). By being pushed and driven to engage local industry, including vocational training, there is some reinforcement of breadwinner status - such as the need to eschew education for financial capital to become a provider (Beasley, 2011; Powell, 2008).

The literature is mixed on if fathers or mothers are more influential in their children's educational trajectory. Some literature has argued that fathers are of particular importance due to their role in the labor market while others have shown mothers are more involved and important others in the rural setting (Ali \& Saunders, 2006; Blackwell and McLaughlin, 1999; Chenoweth \& Galliher, 2004). Regarding the decision to go to college, in sum, the same factors are at play for both young women and men, but tend to drive them into differing directions. The local economy and job prospects remain paramount to the decision-making process. Females see diminished prospects locally while males see more immediate opportunity. Although both mothers and fathers both contribute to the ultimate development of educational aspirations in unique ways, this highlights that gender roles and expectations in rural and Appalachian communities remain common. For young women, they may feel pushed to excel and develop higher aspirations for the pragmatic purpose of finding a good paying job all the while still feeling compelled to participate in the community and fulfill expectations of the family. For women who do not go to college, their social circle becomes more insular, while for young men it expands lending to the notion that other influences and influencers become more credible and present. Although college may be on option, a pressing matter is becoming a breadwinner made possible through the local labor force. This divergence between genders intimates a negotiation 
each student makes regarding future job prospects in light of family connections and assigned or perceived gender expectations.

\section{Aspirations, Identity, \& Obligations to Work}

The literature shows that rural youth have lower educational aspirations than non-rural students (Blackwell \& McLaughlin, 1999; Snyder et al., 2009). However, "within both rural and urban areas there are huge gaps in educational attainment between those who come from advantaged situations and those who do not" (Blackwell \& McLaughlin, 1999, p. 43). Such as, there are differences between the aspiration of rural versus non-rural students, but the difference is more explicable by student's access to resources. Blackwell \& McLaughlin (1999) use metrics like being from white families, having professional parents, as well as school and community make-up to explicate advantage. This tends to support the literature above arguing that expanding social circles for rural youth can mean greater opportunity for interaction with college educated adults and the likelihood of attending college (Brown et al., 2009; Singh \& Dika, 2003). Moreover, this also fits the narrative put forth by Brown et al. (2009) that Appalachian adolescents exposed to poverty do not necessarily have low aspirations, but their aspirations often derail as they reach adulthood.

Howley (2006) makes the argument that when it comes to post-secondary education, both rural and non-rural students aspire to achieve some higher education, but not necessarily to the same level of attainment. Rural students are more likely to want to stop after community college or a four-year degree, while non-rural students are more likely to desire to go further (Howley, 2006; Meece et al., 2013). Part of this may have to do with advanced training being seemingly less applicable in the rural context if they hope to return home (Howley, 2006). Ultimately, Howley (2006) draws a conclusion that parallels Blackwell and McLaughlin (1999) denoting that 
although family and local factors are at play, it is unfounded to believe that, "rural youth limit their aspirations because they suffer from uniquely rural handicaps" (p. 62). This argument is supported by the finding that youth with lower family income also have lower aspirations (Ali \& Saunders, 2008; Meece et al., 2013). This finding helps intimate aspiration is not necessarily tied to rurality per se, but instead more directly connected to the propensity of lower income families in rural settings (Meece et al., 2013).

Friesen and Purc-Stephenson (2016) acknowledged that aspiration is perhaps stymied for rural students because advanced education also means a departure from home for something unknown, creating a conflict with a developed rural identity. The development of a rural identity that is tied to family and community has been denoted in the literature as factors in both not attending college as well as hoping to stay near family (Agger et al., 2018; Friesen and PurcStephenson, 2016; Gibbons, et al., 2020; Meece et al., 2014). For example, Meece et al. (2014) argues that "lower educational and occupational aspirations were reported by youth with strong attachment to a rural lifestyle, more positive perceptions of local economic opportunities, and residential aspirations to remain close to their home community" (p. 11). However, rural youth who favored staying locally intimated greater career expectations compared to their educational aspirations - presenting a mismatch between education aspiration and career expectation (Meece et al., 2013). Aspiration is not stymied purely through growing up in the country, but it is instead associated with economic status and proximity to resources. The argument is not that some rural influences such as social circle structures or the development of an identity are not at play or influential, but instead lack of financial capital and resources at the community and family level influence educational aspiration and the necessity to work. 
For students who do go to college, they are less likely to enroll immediately after high school, and are also less likely to stay enrolled without interruptions (Byun, Irvin, \& Meece, 2015). Hutchins et al. (2012) found in a national sample that rural youth who had high educational aspirations also planned to split time working. Moreover, students who planned to work while receiving an education or did not seek further education at all, were more likely to be in a general or vocational track in high school, more likely to have economic hardships, had lower parental educational expectations, and generally perceived more negative school experiences (Hutchins et al., 2012). Female students were less likely to plan to work, including while seeking an education, while Latinx and Native American rural students had either a greater likelihood of simply seeking to enter the workforce or were unsure if they would pursue education while working (Hutchins et al., 2012). Students, in general, were less likely to go to school while working unless they left their hometown altogether (Hutchins et al., 2012; Snyder et al., 2009). For young adults who do engage the workforce early, they effectively begin a perpetual association with the labor force (Snyder et al., 2009). Powell (2008) opines that for rural Appalachian students, there is a struggle to determine between becoming an adult or going to college. Work is associated with maturing and growing-up, but college is an elongation of youth - a failure to participate in social expectations.

The literature shows that being from a rural locality does not in and of itself put the student at risk for having lower aspirations. Instead, even if in the rural setting, access to privilege and resources are vital for aspiration and attainment. It is just the case, though, that many rural locations, such as Appalachia, have a greater number of low-income families. Moreover, referencing the argument levied above, rural and Appalachian communities also tend to underinvest in educational resources. At the same time, cultural expectations and practical 
reasons lead rural and Appalachian students to feel compelled to work after high school, even if going to college.

\section{High Achievers \& Community Attachment}

There is ample evidence throughout the rural and Appalachian literature that academic high achievers do not disproportionally desire to leave or escape their community of upbringing compared to their peers (Byun, Meece, Irvin et al., 2012; Howley, 1997; Petrin et al., 2011; Petrin et al., 2014). Several large national studies indicate that the highest rural achievers did not have negative feelings toward their community or a strong desire to depart (Byun, Meece, Irvin et al., 2012; Petrin et al., 2011; Petrin et al., 2014). Instead, these students were actually more attached to their home community because this was the context in which they had found support and success (Petrin et al., 2011; Petrin et al., 2014). This led to a desire to seek a college education, but also plans to return (Petrin et al., 2014). These findings of high achievement being connected with a desire to stay or at least return after college actually support some of the already recounted literature above regarding staying versus leaving as an economic decision.

Howley et al. (1997), in comparing students of a statewide honors society in WV versus a sample of students from the general rural population, found that neither group of students indicated a greater desire to leave their local community, and students in the honor society actually felt greater community satisfaction (Howley et al., 1997). However, the most extremely rural of the honors group were found to be less satisfied with their local community than honors students in more populated areas likely due to perceptions of local opportunities (Howley et al., 1997). The literature, both Appalachian specific (Howley et al., 1997) and those with a national sample (Byun, Meece, Irvin et al., 2012; Johnson et al., 2002; Petrin et al., 2011; Petrin et al., 2014), opine that, high achievement does not correspond with an overt desire to leave, and high 
flyers leaving and not returning because of some discordant feeling with their home is not supported. Instead, the decision is more pragmatic in that student's career trajectory simply does not match hometown opportunity (Petrin et al., 2014). Johnson et al. (2002) has argued that lower educational aspirations is actually more associated with a desire to leave the community. However, the challenge is that if lower-achievers have less favorable views of their community, it is more complicated for them to leave due to fewer career options (Foster \& Main, 2016; Petrin, et al., 2011). This harkens back to the notion put forth by Corbett (2005) who argues that education associates with geographic mobility.

However, some gradations surface in the literature creating discontinuity. For example, among rural Appalachian students, those with high college-going preparedness were less concerned about local job prospects, but importantly, did not feel particularly compelled to stay locally (Carrico \& Matusovich, 2016). Also, there may be a difference in the degree of rurality as Howley et al. (1997) found that high achievers from the most rural localities had less favorable local attachment compared to rural students who came from less isolated hometowns. Therefore, the implication is that job prospects in the most rural areas may be so unrealistic, even after going to college, that leaving for good is a foregone conclusion.

\section{Escaping the Stereotypes}

One theme that surfaces throughout the literature is the notion of stereotyping and its impacts on post-secondary attainment. Students feel the weight of these stereotypes and even worry they are destined to be fulfilled while others find them as motivating and a reason for escape (Gibbons et al., 2020). In a study of WV students, those with stereotypical ideas of the state, perhaps originally prescribed by outsiders, also had a strong desire to leave (Towers, 2005). Another study of a college preparatory program found that students from Appalachia who 
failed to identify as such also readily pinpointed stereotypes about their community and region (Arnold, 2017). The students felt they were the exception to those stereotypes (Arnold, 2017). In a study of Appalachian women, rejection of stereotypes such as early pregnancy and reliance on government assistance was a major motivation to attend college (Welch, 2013). Commonly, these stereotypes actually surfaced from educators who intimated that the student would not be able to break the poverty cycle, to which the student rejected (Welch, 2013).

\section{College Integration}

For students who do go to college, many face new challenges as they attempt to navigate the unknown environment and maintain important social ties. In general, rural students are unlikely to seek out and attend highly selective colleges often because they are located in urban areas which are, proximity and culture-wise, quite distant (Byun, Irvin, \& Meece, 2015). Bradbury and Mather (2009) denote that Appalachian students chose their college based upon qualities like being “" 'close to home,' small, and affordable” (p. 269). Staying in the region is a viable option for many students (Bush, 2015). Community college has been regarded as a way of staying in the rural Appalachian community while also gaining an education and perhaps skills for the workforce, although some Appalachian students have denoted a stigma in going to the local community college (Beasley, 2011; Wright, 2012). Choosing a college to simply stay close by, however, has downsides. Powell (2008), who examined the experience at an Appalachian open-admissions college found that students felt somewhat stuck given the institution's options with limited majors (Powell, 2008). They also saw that the college having a large Appalachian population meant the degree may be perceived as less valuable (Powell, 2008).

However, smaller colleges may be more conducive to the integration of Appalachian students (Arnold, 2017; Bush, 2015; Hlinka, 2017). Wilson and Gore's (2009) research rested on 
the notion that Appalachians value a sense of collectivism, and students may acclimate better to a college environment if it promotes that same value (Wilson \& Gore, 2009). The authors confirmed that at least for moderate and high performers, feeling connected to the environment associates with better grades, which was not found in non-Appalachians (Wilson \& Gore, 2009). For Appalachian low-achievers, they actually performed worse as their feelings of connection to the college increased, to which the authors posit may relate to balancing experiences and opportunities with academics while arriving with weak preparation (Wilson \& Gore, 2009).

Practically speaking, students also have concerns about their ability to pay for college, recognizing the need to ascertain funding (Beasley, 2011; Bradbury \& Mather, 2009; Carrico \& Matsusovich, 2016; Chenoweth \& Galliher, 2004; Corbett, 2005; Morton et al., 2018; Powell, 2008; Snyder et al., 2009; Wood, 2012). Cost also forces some Appalachian students to reconsider certain majors, particularly if they will need remedial or prerequisite courses that increase cost and time in school (Powell, 2008). Corbett (2005) argues that cost can be a major driving factor when it comes to deciding to pursue higher education. "A rough life in a known community among family and friends may look better to many youth than taking a very expensive shot at an educational journey that represents an expensive, unproven, and uncertain path" (Corbett, 2005, p. 65). Rural students struggle with the psychological aspects of student debt and tussle with it as a way to pay for an education (Snyder et al., 2009). However, cost and financing higher education may be one hurdle that could be surmounted, but on top of other factors, including for example, challenges to rural identity and leaving the community, it becomes a compounding issue layered on others (Friesen \& Purc-Stephenson, 2016). In general, Appalachian students may struggle to understand basic concepts common in a college setting, such as scholarships and financial aid (Hand \& Payne, 2008). This literature closely parallels that 
of the first-generation students in which just because a concept is perceived as banal by many practitioners in the environment does mean it is as readily understood by all (Hand \& Payne, 2008).

Morton et al. (2018) found that high school students from rural areas actually worry and about fitting in and making-friends on campus. For Appalachian students, the literature has shown that first-generation students are less likely than peers to participate in co-curriculars such as Greek life, clubs, or organizations, but this lack of integration has not shown to necessarily impact persistence (Wood, 2012). Appalachian first-generation students prefer more informal means of interactions as indicative of their desire to be with peers as a positive indicator for their likelihood to persist (Wood, 2012). Relating back to the concept of Appalachian students promoting collectivism, managing these attitudes are important for integration on campus (Gore et al., 2011). For Appalachians, it is important to feel connection to the campus and fellow students. Gore et al. (2011) who studied Appalachian college students compared their study findings to international literature and noted, "members of collectivistic cultures seek to establish connections with others and to maintain their current relationships, a process which sometimes requires some degree of self-sacrifice" (p. 390).

However, aspects of Appalachian culture may make it more challenging to for students to participate on campus. In fact, students who speak with an Appalachian accent or dialect can feel self-conscious in class and concerned others will perceive them as unintelligent or incompetent (Dunstan, 2013; Dunstan \& Jaeger, 2015; Hunley, 2015). These students feel less confident taking science classes with complicated terminology, worry they are stereotyped to certain beliefs, and avoid classes with a lot of required participation (Dunstan, 2013; Dunstan \& Jaeger, 2015). Commonly Appalachian students practice code-switching or essentially leaving their 
accent "on" around peers or circles they were comfortable with—signaling a group associationbut "off" in the academic setting or when they perceived they need a more "mainstream" dialect (Dunstan, 2013). Despite any feelings of alienation, the literature has documented that displaced Appalachians may actually lean on their dialect as a form of cohesion or signaling of cultural inclusion (Jones, 2002; Tang \& Russ, 2007). Moreover, in some instances, Appalachian students actually utilize feelings of stereotyping to add to their motivation to succeed (Gallagher, 2019).

As Appalachians rely on techniques like code-switching to ascertain and establish cultural boundaries, they also shift or obscure specific aspects of their background depending on the moment or situation (Webb-Sunderhaus, 2016). This has been described as choosing what is or is not tellable based upon what the Appalachian student perceives their audience-whether it be faculty or peers - to believe about the culture (Webb-Sunderhaus, 2016). Darling (1999) makes the point that actually, Appalachian students have an innate desire to establish personal connections with others, which makes sense in-light the culture's collectivist tendencies. As was extensively argued in part one of this literature review, college campuses frequently promote individualism, freethought, and personal agency. For Appalachians, the literature shows that students who are comfortable with their own feeling of collectivity on campus actually have higher academic self-efficacy, delineating an ability to manage their identity and abilities in light of higher education valuations (Gore et al., 2011).

Many Appalachian students, particularly first-generation students, struggle to establish connections with both faculty and advisors, which portends to feelings of dislocation or isolation (Bradbury \& Mather, 2009; Darling, 1999). Hlinka (2017) characterized Appalachian firstgeneration students as feeling "betrayed and angry" by advisors when challenges surfaced that could impact, for example, completing major prerequisites or transferring credits (p. 156). The 
author argues, "feelings were magnified by the region's habitus that places importance on trusting authority figures and being confident that others will take care of you" (Hlinka, 2017, p. 156). It is not to say these feelings were permanent, and for many students, they ameliorated as they continued to interact and become comfortable with the environment and processes (Bradbury \& Mather, 2009). In fact, among Appalachian first-generation students, even with less academic engagement than other students (for example meeting with advisors or faculty, engaging in help centers, etc.), some research has shown no connections on year-to-year persistence (Wood, 2012).

Many students stay in close contact with family (Bush, 2015). The ubiquity of cellphones or at least the ability to call home has also tended to a constant medium for communication with those same social circles (Bradbury \& Mather, 2009; Bryan \& Simmons, 2009; Bush, 2015). For commuters, there is seemingly less of a break with home and social connections, which also hinders social integration on campus (Bradbury \& Mather, 2009), and of course, connection to peers and the college environment (Darling, 1999; Wilson \& Gore, 2009). Families of firstgeneration students from Appalachia Kentucky were found to visit their child while at school, but actually kept their distance from the college itself often opting to spend time off-campus-a boundary managed by both the student and the family (Bryan \& Simmons, 2009).

Appalachian students have denoted that the ability to go home can serve as an escape, particularly when struggling to manage new relationships, such as roommates from different background, or experiencing bouts of homesickness (Bradbury \& Mather, 2009). As staying connected may soothe some discontinuity, it can hinder and obstruct the student's acclimation on campus. In fact, the literature has found that families can be both a support system as well as an impediment to integration (Bradbury \& Mather, 2009; Hlinka, 2017). Students often sense a 
feeling of guilt for choosing to participate in academics or extracurriculars instead of being with family (Bryan \& Simmons, 2009; Hunley, 2015; Welch, 2013). Particularly, Appalachian students were often drawn back home to participate in family functions and attend to family needs, while family did not realize the expectations and commitments of being a college student (Hlinka, 2017). Returning home can also mean reassociation with individuals who may have been discouraging of college (Bradbury \& Mather, 2009; Hunley, 2015). Among Appalachian first-generation students it was found that students missed deadlines and academic activities because of returning home to, for example, take care of relatives or work on the family farm (Darling, 1999).

There is sometimes pressure from family for Appalachian students to simply abandon college altogether for jobs with more immediate payoffs (Snyder, 2007; Powell, 2008). Wallace \& Diekroger (2000) found that Appalachian college students often received discouraging remarks, putdowns, or expected to partake in work that took time away from studies. Women reported more general deterrence from college, but when men were deterred, it was based on the notion that they make better money without the degree (Wallace \& Diekroger, 2000). This may seem discordant with literature above that found young women felt more compelled to go to college, however, this references how women are treated when enrolled and balancing school and home. There is also the feeling of women being pushed or pulled to traditional genders roles as a family caregiver, including for some who may already be mothers (Welch, 2013). This discontinuity is exemplary of the mixed messaging that rural students, particularly female students, receive.

Some Appalachian college students credit their distance from home as one of the reasons for their success (Arnold, 2017; Bryan \& Simmons, 2009). In fact, researchers found that 
Appalachian first-generation students who developed a stronger identity as a college student spent more weekends on campus despite the fact that they also recognized closeness of family and home community as important for college success (Bryan \& Simmons, 2009). These findings delineate dueling commitments of being a student but also maintaining a designated role in the family — a form of "bi-cultural competence" which can help students manage and succeed despite pulls from opposite directions (Darling, 1999, p. 189). Many times, Appalachian students want their parents involved when they make a decision related to their future as family values and expectations are central to the decision-making process (Beasley, 2011; Bryan \& Simmons, 2009; Darling, 1999; Hunley, 2015). However, as seen with the literature related to firstgeneration students, many Appalachian first-generation students do not see their parents as necessarily grasping the nuances of many situation, therefore, creating a tension between wanting their guidance but fearing they are misinformed (Bryan \& Simmons, 2009). Nonetheless, evidence shows that as many parents may be uncertain about college in the beginning, their comfortability and support increases (Hunley, 2015). This change has more to do with parent's recognizing their own uneasiness, lack of practical knowledge, and other barriers regarding the experience (Hunley, 2015).

In sum, the literature shows that Appalachian students favor going to college close by, although options for this route may be limited. Given their academic preparation, acclimation may not be easy, either. Though, the transition may be smoother when colleges have certain qualities like being smaller or promoting a feeling of collectivity. Students must also grapple with tasks like ensuring they are able to pay for their education and accessing advising. This is complicated by both not understanding these tasks as well as the psychological struggles, such as taking on debt. For integrating with peers, Appalachian students actually value these new 
relationships, even if they choose to engage them in more informal means. Their desire to make friends and stay connected actually fits with the argument of a collective environment being conductive to integration. Maintaining relationships at home remain important, but also a major challenge as students need to manage the pull from home with new responsibilities, which at times can be at conflict.

\section{Perceptions of Preparation \& Persistence}

The rural literature shows that college-going students, even if showing high levels of achievement, stress and worry about translating ascertained skills and ability to college (Morton et al., 2018). For Appalachians, some "struggle to make the cognitive leap" necessary for college, such as moving away from simply memorizing material to actually learning concepts and ideas (Bradbury \& Mather, 2009; Hlinka, 2017, p. 144). Others have specifically intimated the benefit of courses and instructors explicitly relaying study strategies (Powell, 2008). These assertions reflect the literature above that Appalachian and rural students are more likely to be in educational environments that are less focused on college preparation (e.g., AP courses) and more geared toward vocational training or simply achieving graduation.

An earlier discussion described the educational preparation of rural and Appalachian students, but it also bears indicating how students perceive their preparation upon attending college. The literature shows that many students, looking back, faulted their pre-college educational experiences and teachers for not better preparing them academically (Bush, 2015; Holbrook, 2016; Powell, 2008). Moreover, in some studies, rural students did not always see their educators as beneficial for college going support or preparation (e.g., Arnold, 2017;

Holbrook, 2016; Morton et al., 2018; Powell, 2008; Wood, 2012). Holbrook (2016) argues that 
Appalachian community college students saw their college experience more dependent on their own work and abilities.

Despite no shortage of challenges with going to college, many rural students are able to leverage skills and personal traits to ultimately graduate (Bejarano \&Valverde, 2012; Bradbury \& Mather, 2009; Bryan \& Simmons, 2009; Gallagher, 2019; Holbrook, 2016; Hunley, 2015; Wallace \& Diekroger, 2000; Wood, 2012; Welch, 2013). Gallagher (2019) makes the argument that students retool cultural traits that value personal characteristics such as determination, hard work and persistence to stick it out and succeed. For Appalachian students, others have referenced this as cultivating a strong internal locus of control (Holbrook, 2016; Wallace \& Diekroger, 2000). Wood (2012) makes the claim that Appalachian first-generation students, who have likely relied on a personal self-efficacy to get to college, are able to further invoke confidence to persist. It is important to point out that Appalachia is associated with an embedded and generational poverty that is a challenge for many to escape — an argument levied in chapter one. For many students, a recognition and desire to escape that poverty and associated stereotyping becomes enough motivation to persist and make it through, despite the challenges (Bryan \& Simmons, 2009; Gallagher, 2019; Hunley, 2015; Welch, 2013).

\section{Conclusion}

A major portion of this review has used the backdrop of the stayers versus leavers literature, which has largely pointed out the causes, challenges, and impacts of rural brain drain. The impetus for that backdrop is that the decision to go to college often opens up the opportunity for expanded future mobility. Therefore, it was vital to review that literature before more closely attempting to better understand the path to college for rural and Appalachian students. The literature has extensively analyzed and studied the experience of rural and Appalachian students, 
including how their aspirations is impacted or stoked by their environment. Within the environment, a closed social system that values interpersonal relationships remain important for decision-making. Though as students mature, much of their future prospects turn to the makeup of the local economy. From there, males and females are guided and influenced differently. This dichotomy seemingly pushes some women to college, though not exclusively as many still feel a pressure to ascribe to assigned gender roles. However, it is reductive to say that students only seek or do not seek college based upon local career prospects, because that is not that case. Instead, it is multifactorial and is also influenced by family attachment, rural identity, exposure to certain educational opportunities, and family expectations and structures, to name a few. These are in conjunction with local educational valuing and in Appalachia particularly, the propensity for poverty, which undermines aspiration.

When students do go to college, many students feel underprepared academically, which is perhaps not surprising given the noted educational challenges. In the literature, some students place blame of ill-preparedness on the educational structures and educators while, as already intimated, there is a disconnect between what Appalachian students and their families expect from public education as well as a general under-valuing of rural educational school systems. At college, many balance being drawn back home with a desire to succeed and escape cultural challenges, such as circular poverty and stereotyping. Many fear a loss of group association and concerns of being separated from curated community and kinship circles. Though many Appalachian students actually rely on cultural traits to succeed.

\section{Part 3: Motivations for Rural Practice}

Evidence shows that the decision by physicians to practice medicine in rural areas is multifactorial. Foremost, however, the literature makes clear that having a rural background, 
such as being raised or brought up in a rural setting, is associated with the intent and the ultimate outcome of practicing in a rural community (Brooks et al., 2002; Crump et al., 2016; Herd et al., 2016; Isaac, et al., 2014; Jones et al., 2009; Jones et al., 2014; Kondalsamy-Chennakesavan et al., 2015; Matsumoto et al., 2008; Owen et al, 2007; Playford et al., 2017; Puddey et al., 2014; Puddey et al., 2015; Rabinowitz et al., 2001; Royston et al., 2012; Shannon \& Jackson, 2015; Stagg et al., 2009; Walker et al., 2012; Woloschuk \& Tarrant, 2002). An important distinction in this literature are studies that have pinpointed the impact of rural background or upbringing on intention to practice rurally (Isaac et al., 2014; Jones et al., 2014; Jones et al., 2009; Kondalsamy-Chennakesavan et al., 2015; Royston et al., 2012; Walker et al., 2012; Woloschuk $\&$ Tarrant; 2002) versus those that have examined the actual practice location of graduates (Crump et al., 2016; Matsumoto et al., 2008; Owen et al., 2007; Playford et al., 2017; Puddey et al., 2014; Puddey et al., 2015; Rabinowitz et al., 2001; Shannon and Jackson, 2015). Notably, one study has looked at both aspects (Herd et al., 2016).

An additional nuance is how rural upbringing or origin is determined in the methods of the research. For example, living at a classified rural address for a set number of years (Jones et al., 2014) as well as living in an area with less than 10,000 people (Woloschuk \& Tarrant, 2002) or 50,000 people (Royston et al., 2012) are some of the ways research has attempted to capture rural association and upbringing. Some literature has allowed the individual student or physician to determine if they considered themselves to be from a rural area (Owen et al., 2007; Walker et al., 2012). To highlight this point, Owen et al. (2007) asked physicians if they "grew up rural" leaving the question open-ended. There was a significant association between those who identified as such and ultimately practiced in a rural location (Owen et al., 2007). This finding provides evidence that there is also an identification with growing up rurally that corresponds 
with rural practice as opposed to simply objective measures (e.g., a localities population or density, etc.) (Owen et al., 2007). In addition, when the authors also used objective metrics like rurality of high school and permanent address used during medical school admission to determine rurality, similar associations between upbringing and practice were still evident (Owen et al., 2007). The authors show that both identification and objective metrics may be useful in determining an individual's rural background and its association with rural practice, yet they are not necessarily one in the same (Owen et al., 2007).

The ubiquity of rural background or upbringing as a salient factor in intention to practice as well as actual practice is well established in the literature. Notably, and particularly germane to this project, a study of students of multiple health professions (dentistry, dental hygiene, medicine, nursing, and pharmacy) in WV did not find a connection between a rural background and intent to practice or work in a very rural community defined as a population less than 2,500 (Trickett-Shockey et al., 2013). Though, students with a rural background were more likely to indicate a desire to work in communities of less than 50,000 (Trickett-Shockey et al., 2013). This finding provides evidence of nuances between what rural students see as realistic or viable career locations. Moreover, as levied in the first chapter, health professions, particularly medicine, cluster around larger population centers (Trickett-Shockey et al., 2013). Therefore, it may seem that going back to the most rural community is not realistic, but perhaps staying within the region is a practical alternative (Trickett-Shockey et al., 2013). To add an additional layer, Roseamelia et al. (2014) found that for some medical students of rural origin, they were not as much interested in rural practice, per se, but instead, rural practice served as a default due to the aversion of practicing in cities or more urban areas. 
As much of the literature shows, there is strong evidence of a rural background being connected with rural practice. However, there are also the notion that this goes beyond locality of origination and includes identification with rurality. One study found that many students who were leaning toward rural practice saw themselves as "giving back" or fulfilling a role in a community from which they originated (Roseamelia et al., 2014, p. 263). Although students from a rural background are more likely to practice in a rural community, a nuance in the literature is that there may be distinctions between the size of the community students see themselves working in (Trickett-Shockey et al., 2013). The related literature is given its due in a later subsection, but practitioners are often concerned with provider and professional isolation (Caygill et al., 2017; Roseamelia et al., 2014; Somporn et al., 2018). Moreover, there is evidence that communities with less medical infrastructure see greater physician transience, which could support the notion that there are challenges with being a skilled practitioner in a rural setting with few medical peers (McGrail et al., 2017). In sum, the literature resolves the fact that rural upbringing is important for future rural practice. However, there are other gradations at play, including the size of the community individuals are willing to practice and concerns of being isolated in those communities.

\section{Early Influence}

When it comes to attending medical school and pursing a medical education, there is strong evidence in the literature that exposure to healthcare providers and the general makeup of the local environment is important. Wheat et al. (2015) makes the argument that there is a relationship between both the number of medical students from a county and the volume of local primary care physicians. Moreover, the number of primary care physicians in a county impacts local life expectancy (Wheat et al., 2015). Taken together, being able to increase either medical 
students or primary care physicians can influence the other, and ultimately patient outcomes (Wheat et al., 2015). Although not directly linked to health outcomes, similarly, one study found that the volume of rural medical school applicants was related to not only county ruralness, but also the amount of college graduates and primary care physicians (Anderson et al., 2009).

This aspect of the literature connotates that more providers and practitioners in a rural community can impact the number of aspiring medical students. With the idea of rural upbringing being a salient motivation in future rural practice, more exposure to the healthcare system early would arguably seem to compound that potential. That is, if volume of existing local practitioners can rouse from the population future practitioners, it stands to reason that exposure to the health profession can be dually impactful. There is evidence that early interventions or programs are exceedingly important for the recruitment of rural students (Crump et al., 2014; Gross et al., 2012), including one study that found rural medical school applicants commonly form their aspiration for medical school by age 15 and the greatest influencers are individuals in the school system (Anderson et al., 2009). Moreover, rural students are unlikely to shift or pursue medical education later simply because the prerequisites and metrics needed for admittance can serve as barriers (Anderson et al., 2009). An example of a positive outcome includes a program in rural Kentucky where high school students participate in a summer healthcare shadowing program (Crump et al., 2014). Students who completed the program reported knowing more about local healthcare including providers, had an increased positive perception of local healthcare, showed college preparation as well as academic improvement, and better understood career choices (Crump et al., 2014). Importantly, three-fourths of participants chose a healthcare career, including medicine (Crump et al., 2014). 
As already argued in the previous section, the literature shows educational challenges and limitations for students growing up in rural localities. There is a paucity of studies related to the educational preparation for medical school from the United States. However, Matsumoto et al. (2008) in a study of rural Japanese students found that medical students from rural areas are more likely to have parents with lower educational attainment compared to peers. Rural students were also more likely to have received a public education, typically had lower metrics than peers, but actually showed the greatest academic improvement while in medical school (Matsumoto et al., 2008). These students were also the most likely to practice rural primary care (Matsumoto et al., 2008). Similarly, an Australian study found that rural students are more likely to desire practice in a rural location, arrive at medical school with lower metrics, and often attended public or "government schools" (Puddey et al., 2014). Puddey et al. (2014) posit that the lower metrics of rural medical students creates a catch twenty-two of sorts as, "Very high academic scores generally required for medical school entry may have the unintended consequence of selecting fewer graduates interested in a rural practice destination" (p. 1). To add to the discussion, a second study found that among a group of practicing Australian rural doctors, being rural and rural experiences outweighed entering medical school metrics as predictors of rural practice (Puddey et al., 2015). The authors posit, "Once the influence of a prolonged immersion in a rural clinical environment during undergraduate clinical training as well as the effect of coming from a rural background were considered, we were able to demonstrate very little in the way of a further influence of medical school selection factors or student sociodemographic profiles on the likelihood of ultimately taking up rural practice after graduation" (Puddey et al., 2015, p. 9). 
This portion of the literature points to a few important issues in plans for future rural practice. Building upon the notion that rural upbringing is important for future rural practice, rural students are also commonly academically disadvantaged. Often already having fewer local influencers that include both college graduates and physicians, students need some type of guidance to chart the course to medical school. There is some promise in the investment of community or rural programs to get students on that course. A challenge, though, is that students must be on the path early, already charting a way to build the educational foundation necessary for college and medical school. The evidence shows in section two, though, that many rural students, despite their aspirations, may be less prepared than non-rural peers for college and advanced education. Therefore, the literature points to a clear challenge: recruiting rural students who may be academically disadvantaged or less prepared but more likely to practice in a rural community in the future. These practitioners, in turn, could provide both medical services and educational aspiration. Moreover, more doctors in a community or county can ultimately help increase the number students who set their sights on medical school.

\section{Rural Experiences}

Many students, particularly those who filter in from small regional colleges, attend medical school in more populous areas and, as argued by Crump and Fricker (2015) face an "urban disruption" (p. 422). As the cosmopolitan culture of a medical school can be influential during medical training, rural clinical rotations and experiences can serve as a means to maintain rural connections and identity (Crump \& Fricker, 2015). Rosenblatt (2010) makes the case that, "The persistent shortage of rural physicians is a reflection of the fact that the dominant medical school culture is urban, technologically intensive, and specialty-dominant. Rural physician shortages are in large part due to the systematic bias against primary care that is built into the 
culture, organization, and reward structures on which our medical care system is built" (p. 573). Taken together, these arguments pronounce that there are both cultural and systemic forces that direct students away from, instead of to, rural practice. Types of rural experiences that have been shown to increase either rural interest, aspiration, or actual outcome for students or trainees include pre-college or pre-matriculation programs (Crump et al., 2014), extended time at a rural/regional campus or rural clinical/practical rotations (Crump et al, 2016; Crump et al., 2019; Florence et al., 2007; Isaac et al., 2014; Kondalsamy-Chennakesavan et al., 2015; Playford et al., 2017; Puddey et al., 2015; Rabinowitz et al., 2001; Shannon et al., 2005; Shannon \& Jackson, 2015; Stagg et al., 2009; Walker et al., 2012; Woloschuk \& Tarrant, 2002), rural focused preclinical opportunities (Lang et al., 2005; Wyatt \& Lyons, 2018), rural tracks (Roseamelia et al., 2014), and ultimately, rural graduate medical education training (Brooks et al., 2002; Chen et al., 2010; Dunbabin et al., 2006; Henry et al., 2009; Moores et al., 1998).

Some have argued that that longer duration of rural clinical experience is the most beneficial to foster rural interest (Isaac et al., 2014; Jones et al., 2014; Stagg et al., 2009). This opination would point to the value of medical schools with rural campuses. Crump et al. (2016) have argued that even with other factors aside, including rural upbringing and specialty-type, the greatest influence on future rural practice is attending a rural campus - identified as being in a community of fewer than 20,000. Others, though, have not found such a strong connection between rural practice and rural campuses. Eley \& Baker (2006) argue that by the end of their medical education, most students who attended a rural clinical campus in Australia had still not settled on their long-term career plans. Students, of rural and non-rural background, did report some benefits including more one-on-one time with specialists, but many also lamented seemingly less professional development opportunities and socialization opportunities (Eley and 
Baker, 2006). These challenges are further highlighted below, but this literature ultimately exemplifies that extended time in a rural setting is advantageous, though perhaps not always to the point of hooking practitioners for extended rural service.

Other clinical experiences, typically shorter in duration, have also shown some value. In an Appalachian family medicine elective preceptorship program, more than fourth-fifths of trainees went on to pursue primary care (with the majority in family medicine), and students were much more likely than fellow graduates nationally to practice in rural and underserved areas (Lang et al., 2005). Woloschuk \& Tarrant (2002) found that Canadian students commonly indicated an increase in desire to work in a rural location—including as a locum—after completing a rural rotation on their family medicine clerkship. Of note, students from rural areas often denoted they would likely work in a rural location in the future compared to urban students, but urban students still indicated an increase in stated potential (Woloschuk \& Tarrant, 2002).

Similarly, there is evidence that rural experience in residency training can also impact the decision to practice rurally (Chen et al., 2010). In fact, one study "found that rural residency trainees are over three times more likely to practice in rural areas" (Chen et al., 2010, p. 596). However, there are so few rural residencies that actually few rural practitioners are rurally trained during graduate medical education (Chen et al., 2010). Chen et al. (2010) found that only about $5 \%$ of rural physicians with a doctorate of medicine (MD) and $10 \%$ of rural physicians with a doctorate of osteopathic medicine (DO) were trained in a rural setting. This would support the idea that medical school serves as a disruptor for the trajectory to return to rural locations as there are seemingly few options to train rurally at this stage of medical education. In sum, though, the literature shows that many rural experiences can be influential in directing future 
rural practice. It also shows that by adding a rural rotation, students may benefit from, for example, an optimized clinical learning environment, but it does not always translate to students walking away with a settled career trajectory. Additionally, not all students are given an opportunity to train in a rural setting, instead, many rural doctors practice without that experience.

\section{Educational and Clinical Benefits versus Drawbacks of Rural Experiences}

The literature shows that deliberate rural educational and clinical training experiences throughout the medical education continuum often promote positive aspects of rural practice, are not educationally detrimental, and in fact, provide unique training experiences (Caygill et al., 2017; Florence et al., 2017; Haggerty et al., 2019; Henry et al., 2009; Maxfield et al., 2015; Roseamelia et al., 2014; Shannon et al., 2005; Smith, 2012; Somporn et al., 2018; Wyatt \& Chater, 2018; Wyatt \& Lyons, 2018). Underpinning this argument is the idea that by going to a rural location for training, there is more opportunity for one-on-one training with attending physicians, more direct patient care, and often continuity among patients in certain clinical learning environments (Caygill et al., 2017; Crump et al., 2019; Eley and Baker, 2006; Maxfield et al., 2015; Wyatt \& Lyons, 2018; Zink et al., 2010). Rural experiences, among benefits highlighted below, can promote clinical skill self-efficacy, communication skills, interactions with a specific community, and generally feelings of greater social responsibility (Caygill et al., 2017; Crump \& Fricker, 2015; Henry et al., 2009; Maxfield et al., 2015; Roseamelia et al., 2014; Shannon et al., 2005; Wyatt \& Lyons, 2018).

There is evidence that rural experiences in the clinical phase of medical school improves clinical performance in some areas. Among rural experiences, rural longitudinal clinical experiences in the third year are among the most frequently cited (Somporn et al., 2018). In an 
example of one of those experiences, students who completed a rural nine-month longitudinal clerkship curriculum performed differently on some aspects of the institutionally developed Observed Structured Clinical Examination (OSCE) (Zink et al., 2010). ${ }^{15}$ The rural students were better at establishing rapport with patients and certain aspects of clinical encounters - indicating clinical skills (Zink et al., 2010). However, a downside for rural longitudinal students was the need to view recorded lectures after they were presented live at other sites, which many students failed to do in lieu of more robust clinical experiences (Zink et al., 2010). As result, rural students performed lower in certain content areas of the OSCE despite clinical gains elsewhere (Zink et al., 2010).

Rural clinical rotations are well documented in the literature (e.g., Crump et al., 2019; Maxfield et al., 2014; Wyatt \& Chater, 2018, p. 266). For example, a group of students from an urban area were selected at random to complete their surgery rotation at a rural site in Kentucky (Crump et al., 2019). After the rotation, these students had more favorable views of living and practicing in a rural community, including positive views of community qualities (Crump et al., 2019). Students at the rural site also performed statistically similar to those who completed the clerkship in an urban setting on the National Board of Medical Examiners Surgery Subject Examination (Crump et al., 2019). Added benefits of the rotation included being able to work more directly with the attending surgeons indicating a more one-on-one clinical experience (Crump et al., 2019). Besides the outright clinical skills benefit, there is also evidence that an important aspect of rural training experiences is actually getting to know and becoming

\footnotetext{
${ }^{15}$ The authors explain the longitudinal experience as, "(1) 9 months in one location compared with 8 weeks, (2) continuity in one hospital/clinic location, and (3) the same primary care preceptor for 9 months. Students do not change locations every 4 to 8 weeks; their primary care and required specialty clerkships are in the same location for 9 months. They are encouraged to spend a half day a week with their primary preceptor when completing their other clerkships requirements" (Zink et al., 2010, p. 709).
} 
comfortable with the rural community (Florence et al., 2007). One study of Appalachian medical, nursing, and public health students who participated in a three year long educational experience that facilitated rural community educational experiences found that participants had an increased interest in participating in rural and primary care (Florence et al., 2007). They were also more interested in working with underserved or disadvantaged populations (Florence et al., 2007).

Maxfield et al. (2014) found family medicine clerks at rural sites received overall higher course grades, which was largely resultant from clinical evaluations from rural preceptors. It is possible that students performed better because rural preceptors have less experience with medical student evaluations and in turn give higher ratings (Maxfield et al., 2014). On the other hand, rural experiences are often intensive and students spend more time with individual faculty, giving them more opportunity to receive feedback and ultimately improve skills throughout a rotation (Maxfield et al., 2014). Finally, an Australian study found that third year medical students who are assigned to a location governmentally designated as "Very Remote Australia" for six-week rotations actually performed higher than students at other classified locations in, among other areas, clinical evaluations and case-based presentations (Wyatt \& Chater, 2018, p. 266). The aforementioned evidence points to the fact that students may receive beneficial and dense clinical exposure on rural rotations. However, students may also receive higher clinical evaluations in rural settings due to preceptor inexperience or limited volume of rotating students (Maxfield et al., 2014). In a separate study, rural clinical preceptors reported, among other things, being unsure of what, educationally, the rotation should entail and at what level students should be performing (Baker et al., 2003). The point is that there is documentation of medical students performing better clinically in rural settings. However, the literature leaves unresolved if 
that is a result of one-on-one and intensive rural experiences or because rural preceptors may evaluate students differently than faculty at clinical facilities with larger volumes of learners.

Although there is ample evidence that rural experiences do provide enriching experiences and may encourage some students to lean towards rural practice, there are also some notable downsides that seemingly could impact recruitment. For example, students who complete an extended rural experience are often more likely to denote they felt social isolation or were afraid that rural practice could mean isolation (Caygill et al., 2017; Roseamelia et al., 2014; Somporn et al., 2018). Part of that isolation was related to lacking social support from fellow medical students while on rotation (Roseamelia et al., 2014). There are also nuances between how both urban and rural students viewed the potential isolation, which was a particular concern of students from more urban backgrounds (Walker et al., 2012). Other students have been concerned with seemingly limited career advancement, professional development opportunities, family separation, and opportunities for a spouse or significant other (Eley et al., 2006; Henry et al., 2009; Roseamalia et al., 2014; Walker et al., 2012). Regarding opportunity for experiences, one study of rural surgical trainees in the United Kingdom were found to have less scholarly productivity than counterparts elsewhere, but instead did get more opportunity to participate in certain surgical procedures (Brown et al., 2018). This finding would support the argument that rural clinical training does provide more intensive clinical experience, but perhaps at the cost of other professional activity.

There is also the notion from the literature that students with a rural background or who are already geared toward rural practice perceive rural experiences and future opportunities differently than peers from urban backgrounds. For example, WV medical students participating in a rural rotation who afterward intimated an intention to practice rurally had more positive 
perceptions of the rotation, were more likely to want to practice primary care, and showed greater interest in providing care to destitute or underserved populations (Shannon et al., 2005). Additionally, a study of Australian students who completed a rural clinical semester found that students who had a preference for rural practice perceived greater opportunities for their future career compared to students who preferred an urban career (Walker et al., 2012). For students looking to an urban career, they were much more likely to also see rural practice as isolating (Walker et al., 2012).

In sum, the literature shows a number of benefits of rural experiences including stimulating interest in rural practice. Rural clinical experience can also provide unique experiences to work with rural populations and communities. There are implications for the development of clinical skills as the experiences have fewer learners in the clinical environment and more chances to work directly with preceptors. Rural experiences, though, can also come with downsides, which include spotlighting aspects of rural practice like greater potential for isolation or limitations, both professional and personally that can cause some learners to see future rural practice as less favorable. However, the literature also shows that origin, including either rural or urban, can actually influence how students perceive those rural experience.

\section{Rural Backgrounds versus Rural Experiences}

There is evidence, as presented above, that rural experiences are educationally beneficial and may help some students choose a rural practice. However, these experiences are also resource intensive and there are a multitude of independent factors that can also be influential to the individual student (Ranmuthugala et al., 2007; Somporn et al., 2018). There is some discord in the literature regarding which type and to what degree rural experiences tip the scales and actually turn students to rural practice (Ranmuthugala et al., 2007). The literature points to the 
idea that rural experiences are beneficial for recruiting rural providers, but in what ways do they buttress already preconceived ideas about rural lifestyle and practice (Barrett et al., 2011)? In other words, are these experiences actually persuasive for students without a rural predisposition? For example, reconsidering the elective Appalachian rural preceptorship described above (Lang et al., 2005), were the students that completed the program and ultimately worked in a rural setting already on track to do so regardless of the experience? Brooks et al. (2002) framed this question as the classic nature versus nurture dilemma. This question is a notable tension in the literature.

A group of studies of Australian rural experiences highlight this unresolved matter. Jones et al. (2014) found that other factors aside, including rural educational experiences, students were mostly motivated to practice rurally if they arrived at medical school with that mindset and if they had a rural background. Meanwhile, Walker et al. (2012) argues that being from a rural background is important but is tapped into with a clinical rural experience. Similarly, Playford et al. (2017) makes a near identical argument, with the addition of rural students needing to arrive with some desire to work in a rural area, and it is the rural experience that keeps the spark alight. This notion goes handedly with the argument above that many medical schools are aligned with population centers and away from rural communities, and therefore rural programs have a vital role to play in maintaining identity or interest. Finally, some have shown that longer rural clinical education as well as having a rural background increased the likelihood of rural practice, particularly when both factors were present (Kondalsamy-Chennakesavan et al., 2015).

To add another layer, Herd et al. (2016) found that among year three residents, those who started medical school stating they preferred to ultimately practice in a rural setting were still likely to report a desire to do so-regardless of their community origin. As summarized by the 
authors, "These findings suggest that the effect of preferred practice location at medical school commencement is independent of, and enhances the effect of, rural background" (Herd et al., 2016, p. 18). This study shows that rural origin is important, but also creates a tension with the arguments above that rural experiences are vitally important to stoke intention for rural practice. For example, if a student arrives with predetermined ideas regarding their future practice, and those intentions remain into residency, were any required rural experiences along that way beneficial to the aim of promoting rural practice? If students do arrive with this preconceived direction, it would lend some credence to the importance of rural precollege experiences as among the most beneficial for sparking favorable views of rural practice (Anderson et al., 2009;

Crump et al., 2014).

\section{Specialty Choice}

In conjunction with the argument above that rural origin impacts the decision for rural practice, the literature shows that rural origin is not only a predictor of practicing in rural location, but also increases the likelihood or desire of becoming a primary care physician (Matsumoto et al., 2008; Shannon et al., 2005). Of American medical school graduates, nearly a quarter of family medicine doctors enter rural practice followed by general surgeons, internists, and pediatrics as the next most common (Chen et al., 2010). Family medicine, internal medicine, and pediatrics are commonly regarded as primary care specialties. ${ }^{16}$ A study of WV medical students found that those with a desire to practice in primary care were more likely to ultimately

\footnotetext{
${ }^{16}$ The American Academy of Family Physicians denotes that, among other definitions provided, "A primary care physician is a specialist in Family Medicine, Internal Medicine or Pediatrics who provides definitive care to the undifferentiated patient at the point of first contact, and takes continuing responsibility for providing the patient's comprehensive care. This care may include chronic, preventive and acute care in both inpatient and outpatient settings. Such a physician must be specifically trained to provide comprehensive primary care services through residency or fellowship training in acute and chronic care settings" (American Academy of Family Physicians, 2020, Definition \#3).
} 
practice in a rural location (Shannon \& Jackson, 2015). However, splicing all primary practice specialties together as predictive or associated with rural practice is not always clear-cut. For example, Rabinowitz et al. (2012) found that students favoring a family medicine career were more likely to practice rurally. They were also more than one and half times as likely to practice in rural areas compared to students preferring, among others, general surgery, psychiatry, emergency medicine and internal medicine as well as more than two times as likely to practice in rural areas compared to students desiring, among others, anesthesiology, obstetrics and gynecology, pathology, radiology, and notably, pediatrics (Rabinowitz et al., 2012). The authors add, "although medical educators and policy makers often combine all primary care physicians into a single category because they all provide generalist care, our findings show that medical students who plan to become family physicians, general internists, and general pediatricians have very different likelihoods of practicing in rural areas" (Rabinowitz et al., 2012, p. 1089).

Several studies of Australian medical students found that incoming students who desired to be a specialist were less likely to desire or intend to work as a rural doctor, while those seeking to be a non-specialist or generalist had greater interest or intent in being a rural doctor (Jones et al., 2009; Puddey et al., 2014; Walker et al., 2012). The aforementioned terms, such as generalist and non-specialist, were left undefined in the literature (Jones et al., 2009; Puddey et al., 2014; Walker et al., 2012). These findings are similar to studies of American medical students that have drawn similar conclusions. Owen et al. (2007) found that matriculating medical students that intimated a desire for a family medicine, general internal medicine, or general pediatrics was predictive of eventual rural practice. Meanwhile, others have shown an association between medical students desiring to practice family medicine and rural practice 
(Rabinowitz et al., 2001; Roseamelia et al., 2014) and students seeking a family medicine residency and rural practice (Crump et al., 2016).

Stagg et al. (2009) found, among Australian medicine post-graduates who completed a one-year rural program during medical school, that a multitude of factors, including rural versus urban background and preference for either general practice versus a specialty were at play in deciding where to practice. First, some students who favored general practice in a rural setting commonly had a rural background and were often partnered with someone of a rural background (Stagg et al., 2009). These students attended medical school seeking general practice that was further fostered through their rural experience (Stagg et al., 2009). There were also a cohort of students with a rural background who after starting medical school leaned toward specialty practice and saw limitations in their career by staying rurally, supporting the notion of a disruption, or students being pulled away (Crump \& Fricker, 2015). Among urban students seeking rural practice, many were actually single and more commonly female, and were also less interested in living in an urban area (Stagg et al., 2009). This finding is similar to Roseamelia et al. (2014) who found that it was not that some students necessarily seek rural practice, but instead some simply do not prefer urban practice. Among urban students planning for urban practice, many were male and married, and despite participating in the year-long rural experience, saw rural practice as impractical and worried about career opportunities (Stagg et al., 2009).

The literature shows that the pursuit of a primary care specialty causes many students to lean toward rural practice and the pursuit of non-primary care practice to lean to urban practice. However, there are a multitude of factors for consideration. First, not all primary care is the same with some evidence that certain career choices, such as family medicine, may be more closely 
linked to rural practice than either internal medicine or pediatrics. Secondly, many students may arrive at medical school with preconceived ideas of their specialty choice that may not change throughout medical school. Finally, as Stagg et al. (2009) shows, many students arrive with a desire to practice in certain fields and in certain locations. Rural experiences may or may not influence those students to practice rurally, because other factors may include their own upbringing, perceived ideas about specialty locations, being partnered as well as the partner's background.

\section{Personal \& Professional Factors}

As broached in the previous section, it is possible to examine how important rural background, rural experiences, or specialty trajectory may be in eventual rural practice. However, personal and professional aspects cannot be overlooked as important. McGrail et al. (2017) found that physicians were less likely to stay in rural counties that did not have a local hospital, already had few primary care doctors, and were among the most rural, while local SES and other demographics did not seem to influence whether a doctor stayed in or left a community. These findings seem to point to that idea that perhaps concerns about isolation or professional support denoted in the literature is not necessarily unfounded. For example, if there are no other providers or a hospital in place, the issue of rural practice is all the more challenging. This is more pronounced among those without a rural background as metropolitan born rural physicians were much less likely to stay in the rural setting compared to those physicians born in a rural location (McGrail et al., 2017).

The financial aspect of deciding to practice rurally does surface at numerous points in the literature. There is evidence that programs which specifically grant or offer some type of loan payback or other financial incentive can motivate a student to practice rurally (Kondalsamy- 
Chennakesavan et al. 2015; Royston et al., 2012; Walker et al., 2012). There is also indication that students who receive a scholarship while in medical school, including both with an obligation to work rurally as well as without that stipulation, are more likely to report an intention to work rurally (Dunbabin et al., 2006; Jones et al., 2009). Meanwhile, students who are being financially supported by their parents are less likely to intend to practically rurally (Jones et al., 2009). Regarding employment, at least one study has shown that concerns about finding a job in a rural area may be a limiting factor as students think ahead to potential career opportunities (Roseamelia et al., 2014). Part of that concern may relate to ensuring the ability to pay off debt accrued through their extensive education (Brooks et al., 2002).

In general, the literature has shown that fewer women compared to men choose rural practice; however, there is evidence that women's presence in rural localities is improving (Chen et al., 2010). Similarly, women doctors, of essentially any age, are less likely than men to stay in rural locations permanently (McGrail et al., 2017). Some research has found differences between how male and female providers perceive rural practice, though. For example, women, compared to men, have shown that they expect to make less money by working in a rural setting compared to urban areas, while at the same time intimating part-time opportunities as attractive features to working rurally (Spenny \& Ellsbury, 2000). Women also, compared to men, have more concern about "professional isolation" and "lack of privacy" by living in a rural community (Spenny \& Ellsbury, 2000, p. 183). Others have argued that rural female physicians' value being able to build friendships and relationships with individuals in the community, which portends the idea of an identified community purpose (Paladine et al., 2020). As purpose may be an important factor, so is ensuring similar cultural and political beliefs (Paladine et al., 2020). Matched values and a 
community purpose, then, support the argument that having a rural background may make a provider more apt to practice in a rural location.

Just as with the physician themselves, having a spouse with a rural upbringing has shown to be important in increasing the likelihood that a practitioner will choose a rural location (Royston et al., 2012; Stagg et al., 2009). Rural upbringing aside, a number of studies have shown that a common concern about rural practice is finding suitable career options for a partner or spouse (Henry et al., 2012; Paladine et al. 2020; Spenny \& Ellsbury, 2000; Walker et al., 2012). Prospective and active rural female physicians have specifically indicated that available and relevant work for their spouse was an important consideration in practicing in a rural area, while this was less of a reported factor for male providers (Paladine et al. 2020; Spenny \& Ellsbury, 2000).

Many of the aforementioned factors are what Paladine et al., (2020) refers to as "community integration" (p. 113); such as, the ability to form and maintain relationships within the community, establish a role as a provider, and ensure that a spouse or partner has comparable integration. A nuance in the literature is that many of the studies mentioned in this section are related to why or how women physicians arrive at a community or integrate. There is a paucity of literature that more closely examines the rationales and patterns of male physicians; however, a sensible reason for this limitation is that there are fewer female rural providers and female providers are less likely to stay (Chen et al., 2010; McGrail et al., 2017). Therefore, there is a more pressing need to understand why women physicians may be less likely to work in a rural setting compared to men.

There are a few remaining nuances in the literature regarding certain aspects of an individual's personality that may direct them toward rural practice. For example, in a study of 
Appalachian healthcare profession students (including medicine), those who were more service orientated were also more likely to indicate they desired to practice rurally (Shannon \& Jackson, 2015). Some research has shown that students who are more extraverted as opposed to introverted had greater intentions to practice in a rural setting (Royston et al., 2012). Additionally, one study found that students who had a more positive perception of rural practice also had more positive views or ideas about interdisciplinary practice and working with other healthcare disciplines (Trickett-Shockey et al., 2013).

In sum, the literature shows that beyond rural background, experiences, and career path, there are also personality traits, professional desires, and community factors that are important for both practicing and staying in a rural place. Similarly, the financial aspect of rural practice is an important consideration. Future and nascent practitioners must think of career longevity in light of factors that include the cost of their education and working in a community with the infrastructure to meet their goals and aspirations. Of course, this is balanced with other aspects of their life, including having a significant other who is able to work within the community, has a rural background and/or is comfortable in a rural setting. These are not insignificant considerations and in fact, it would be reasonable to believe that anyone on a professional career path outside of medicine may also pay attention to these various aspects of their life.

\section{Conclusions}

With all the aforementioned literature in mind, evidence shows rural background as well as rural experiences can be important, notwithstanding exposure of some rural drawbacks. As to which experience types are most beneficial is a matter of debate. Rural background being important points to the need for earlier interventions and guidance to the healthcare opportunities in a rural community. Meanwhile, some longer experiences later in medical school have also 
shown merit, including opportunity to practice and hone clinical skills in a more intensive environment with fewer fellow learners. Of note, though, the literature shows that some students who commence medical school on a rural trajectory may be unlikely to change course, which challenges, in some respects, the argument that medical school serves as a disruptor.

Nonetheless, some students do peel away for urban practice and specialist roles elsewhere. Rural experiences do seem to help keep students engaged with practicing rurally, and if nothing else, provide opportune clinical environments which leave many students with a greater appreciation and awareness of rural practice. The debate between which experiences are most beneficial to influence doctors to become rural providers is unsettled in the literature, despite the possibility that there is likely more than one answer. Even more, the literature shows that there is not just a decision to made that includes preferring rural practice versus urban practice. Instead, students face this decision with personal considerations in mind, including fitting into the community, having a purpose, and what is best for their family. How all these factors ultimately work together is still a matter of debate and unresolved in the literature.

\section{Part 4: Medical Students from First-Generation Backgrounds}

Considering the literature presented in section one, there may be some expectation that first-generation students are more familiar and perhaps comfortable in the college environment by the time they matriculate to medical school. However, despite success and proven academic capabilities needed to be accepted into medical school, it does not necessarily mean that all vestiges of discomfort dissipate. In fact, first-generation students who enroll in medical school are still not always well positioned to transition through the experience, and in some ways, continue to face many of the challenges they did as an undergraduate. The literature presented below shows that first-generation students who go on to medical school are in some ways 
already culturally disadvantaged compared to certain peer groups. For example, there is evidence that many medical students disproportionally come from families with physicians or other health professionals, such as psychologist (Tran et al., 2017). Medical background aside, in the United States, most medical students come from high income backgrounds as around $75 \%$ have families who fall into the two highest deciles of annual wage earners (Jolly, 2008). About one out of twenty medical students come from the lowest family income decile-less than around $\$ 20,000$ a year (Jolly, 2008). In other words, incoming medical students are disproportionality from higher income families. For students who come from the lower end of the income spectrum or are firstgeneration students, the medical school experience is quite different as is explicated in this literature review section.

\section{The Path to Medical School for First-Generation and Low SES Students}

Well before arriving at medical school, there is evidence that students from firstgeneration or low-SES families are conditioned to view the medical education career route differently than their peers from continuing generation or high-SES backgrounds (Greenhalgh, et al., 2004; Southgate, et al., 2015). A study of "academically abled and scientifically oriented" UK teenagers "found that their views of seeking a medical career were largely aligned with SES status and not by sex or ethnicity" (Greenhalgh et al., 2004, p. 1). Working-class males were found to be bonded in their peer group showing a strong social identity (Greenhalgh et al., 2004). They also lacked confidence in their academic abilities and were skeptical or held pessimistic views regarding academic “enrichment initiatives” (Greenhalgh, et al., 2004, p. 3). ${ }^{17}$ Additionally, low-SES students showed interest in the monetary gain of becoming a physician,

\footnotetext{
${ }^{17}$ Greenhalgh et al. (2004, p. 3) denote, in this instance, working class background to correspondent with a "head of household" who is unemployed or employed with a routine or semi-routine job. The authors note that students who fit this category were typically distinguishable by actions and behavior including dress, accents, and at times being outspoken, interjecting (with humor), and portraying disinterest or academic inability.
} 
but had superficial understandings of the career and indicated that students of high-SES or with certain educational opportunities were better suited for the career (Greenhalgh, et al., 2004). Meanwhile, high-SES students saw medicine in terms as a possibility among other options, and more commonly considered career options in terms of personal fulfillment (Greenhalgh et al., 2004). They also had a better understanding of how to pursue a medical degree and denoted role models or interactions with the healthcare system (Greenhalgh, et al., 2004).

Similarly, Southgate et al. (2015), sought to better understand the "barriers and enablers to medical education amongst academically able high school students from a range of socioeconomic backgrounds and those of FIF [first-in-family] status" in Australia (Southgate, et al., 2015, p. 74). ${ }^{18}$ The authors utilized Appadurai's capacity to aspire theory, which specifies that although there may be individualistic ambitions or motivations to be successful, ambitions are often constrained by culture, geography, systemic boundaries, and other limitations (Southgate et al., 2015). Many of these limitations are often challenging for the individual to foresee or circumvent. Students from lower SES background recognized a need to follow certain academic trajectories (high grades and test scores) but had little understanding of how to practically pursue medical education (Southgate et al., 2015). Key barriers of low-SES or first-generation students included navigating the admissions process as well as the student's ability to academically prepare for a medical education as schools with larger volumes of low-SES students had greater demands on vocational education as opposed to upper-level math and sciences (Southgate et al., 2015). Central to this point is that students from low SES backgrounds placed higher value on hands-on or personal work experience when considering the skills needed for medical education showing a misunderstanding of the actual academics required (Southgate et al., 2015).

\footnotetext{
${ }^{18}$ Southgate, et al. (2015) define first-in-family as students without an immediate family member to hold a college "qualification" (p. 74).
} 
How first-generation students and low-SES students receive support and capital from their parents and educators is also quite different than students from more privileged backgrounds. Bassett et al. (2018) who examined first-in-family British medical students argues students intimated that they received moral support from family and their parents were happy to hear of their acceptance to medical school. With respect to educators as influencers on the decision to pursue medicine, the literature is mixed. Some first-generation students were discouraged from medicine based on "required intellectual prerequisites" and rigor, to which some instead leveraged for motivation and aspiration (Bassett et al., 2018, p. 5). One study indicated this practice as sorting out who may be "doctor material" as opposed to motiving and stoking aspiration for some students who may be unaware of the medical career path (McHarg et al., 2007, p. 815). Nonetheless, in other instances, first-generation students indicated support from teachers as well as peers, such as older students who had already been admitted to medical school and made long-lasting positive impressions (Bassett et al., 2018).

The literature shows that many first-generation students or those from a low-SES either have only formal interactions (e.g., receiving care) with the medical system early as opposed to less formal associations such as family or mentors who are medical professionals (Bassett et al., 2018; Bassett et al., 2019; Brosnan et al., 2016). Brosnan et al. (2016) points out that these issues resurface later in medical school as some students feel they do not have professional connections like their peers from affluent or physician backgrounds. There are other complications for many first-generation students when it comes to gaining the exposure or experience needed for medical school as one study found that their seemed to be distinct advantages of knowing or having family connections to physicians during the application cycle (Bassett et al., 2019). The implication is not that students benefit from nepotism, but instead are better positioned to have 
received the experiences and opportunities seen as beneficial in the admissions process. The authors highlighted one student who had to travel an extended distance to complete physician shadowing experiences to improve their application (Bassett et al., 2019). The student could not acquire shadowing experiences locally for various reasons, but they felt that having a personal connection would have ameliorated this challenge (Bassett et al., 2019).

Mathers and Parry (2009) looked closely at why attempts to broaden access for underserved populations, particularly students from low SES backgrounds, has not always led to more students actually going to medical school. The authors argue that medicine becomes something outside of student's "imagined futures" as "Institutional and familial habitus provide the context within which the decisions of working-class students are moulded; the layered support mechanisms that contribute to norms, expectations and behaviour are very different to those experienced by capable middle-class students" (Mathers \& Parry, 2009, p. 226). This argument is supported by the literature here. Regarding the direction to medical school, for example, one study denoted that students were skeptical of opportunities to engage with career development activities (Greenhalgh, et al., 2004), while another found that higher education engagement was superficial and failed to present clear roadmaps for student access (Southgate et al., 2015). Some students denoted the value of structured college visits, but the information provided was not directive on how to actually enroll in college (Southgate et al., 2015). In addition, although families may be supportive of seeking out an advanced education like medical school, the support is perhaps sincere but not necessarily practical. Students of higher SES status or from a physician family are more likely to have access to certain social and cultural capital that would, though, make paths and directions more implied and apparent. 
In sum, the literature shows that students from first-generation backgrounds may have access to medical school information and pathways through their school system, but they are not always well positioned to recognize or leverage it appropriately. Moreover, although some students may find early educators as inspiring and beneficial to their success, the literature shows that they also may be subjected to educational sorting based upon perceived ability. These populations of student are also exposed to the expectations and availability of their immediate surroundings including what is offered by their school. School offerings are likely based off of resources available and what is valued in the community. Regarding family, many firstgeneration students lack associations and inherent mechanisms to facilitate the medical school career route. Taken together, the literature shows that these issues compound and establish what first-generation students may see as possible or practical paths, which includes deciding if medical school is unreasonable or impractical.

\section{The Transition to Medical School for First-Generation Students}

Luke (2003), in a central piece, focuses on Australia's junior doctors (post-graduate years one and two) and finds there is a clinical aspect of becoming a physician that relies on a process of socialization. As nascent doctors move into the medical field, they acquire and hold capital, which may be association with other physicians, behavior, knowledge, or practice specialty (Luke, 2003). Underlying the field is the organizational structure of medicine with senior attending physicians at the top of the hierarchy holding decision-making authority, which flows downward (Luke, 2003). Of note, Bourdieu (1993) inculcates, "habitus is a product of conditionings which tend to reproduce the objective logic of those conditionings while transforming it" (p. 87). The medical habitus, as presented by Luke (2003), embodies practices and norms perceived as beneficial and necessary by those practitioners in the medical field for 
utilization and replication. Physicians, particularly those with the greatest perceived knowledge, experience, legitimacy, and credentials, uphold customs and guide the field (Luke, 2003). As such, "Junior doctors, for example, are learning to acquire medical cultural practices, to inherit, and to develop the cultural capital from the profession through their workplace practices" (Luke, 2003, p. 58). In other words, any individual partaking or transitioning into the medical field brings their own capital, which is utilized to navigate the field. Repetitive actions and processes are normalized and buttress a medical habitus (Luke, 2003).

Nicholson (2013) builds heavily upon Luke's (2003) framework. The author denotes that students from non-traditional and low-SES experience the medical field and habitus differently than their peers (Nicholson, 2013). For example, students from underrepresented statuses (including socioeconomic, ethnic, religious, or cultural statuses) struggle to socialize or connect with their peers from affluent or seemingly "traditional" backgrounds (Nicholson, 2013). The same can be true with faculty-student interaction (Nicholson, 2013). Differences are in part a result of varied backgrounds and life experience which fail to generate commonalities (Nicholson, 2013). A failure to thrive can have implications for the development of networks that inhibit formation of cultural and social capital, and moreover, students may fail to see the benefit of socializing as it relates to the development of a professional identity (Nicholson, 2013; Nicholson \& Cleland, 2017).

Beagan (2005) argues that working-class students do not necessarily lack social and cultural capital, "Rather, the social networks, habits, values, expertise and ways of being that are valued in working-class settings are actively devalued within the middle-class norms of medical school" (p. 783). Such as, there is an inherent classism in medical school that expects students more from working class backgrounds to conform to middle-class standards (Beagan, 2005). 
Examples include fellow students referencing or joking about aspects of working-class patient's lives, which can serve as "microaggressions" or "status-reminders" even if they are subtle or indirect (Beagan, 2005, p. 783). Working class students are likely to feel a chasm forming with home relationships while also struggling to build new relationships in a culturally foreign habitat (Beagan, 2005). Brosnan et al. (2016) argue that access for medical students has expanded to be more inclusive of first-generation students, but the experience for students is largely unchanged. Students in medical school must manage a "dual identity... one identity that fits into the world of medicine and one that remains true to the student's social and cultural background" (Brosnan et al., 2016, p. 848). The authors quote Bourdieu (1999) in noting that, "these students were 'excluded from the inside': they were admitted to medicine, but not included in it" (Brosnan et al., 2016, p. 849). Just as with undergraduate first-generation students, the concept of dual identity surfaces elsewhere in the medical education literature (Bassett et al., 2019; ConwayHicks \& de Groot et al., 2019; Southgate et al., 2017).

Medical school may be one of the first times that students start to reflect on their own social background, feel "out of place," or have a greater realization of their own social standing (Bassett, et al., 2018, p. 7). For example, in several studies, first-generation students and lowSES students recognized that many of their high-SES peers received a private education, emblematic of their background (Bassett et al., 2018; Beagan et al., 2005; Southgate et al., 2017). As Brosnan et al. (2016) posit, lacking economic capital can also serve as a barrier to creating connections and social relationships as limited expendable funds can stymie opportunities for participation in activities valued by higher SES peers. "This had the potential to subtly maintain social divisions among medical students, limiting the development of different types of social capital for both poorer and wealthier students by creating separate social groups within one 
cohort” (Brosnan et al., 2016, p. 849). However, students do not necessarily completely sequester themselves into subdivisions, but instead tend to integrate with select groups often more closely associated with their culture and social origins (Bassett et al., 2019). In a study of Australian first-generation students in medical school, students were often aware of which classmates had physician parents and denoted they were less likely to regularly interact with these students (Brosnan et al., 2016). Bassett et al. (2019) argues, though, that by the time students in their study graduated medical school, some first-generation students had actually overcome feelings of social sequestration and were much more comfortable interacting with peers from even affluent or physician families.

The literature shows that first-generation students often engage a type of "tactical incorporation" that includes participating in new aspects of an emerging identity while holding on to facets and vestiges of their upbringing and social background (Southgate et al., 2017, para. 72). For example, one student in an Australian study denoted that although they felt they were "slightly more refined," they strove to maintain relationships with family and sought to use insider knowledge of their culture to connect with patients (Southgate et al., 2017, para. 66). Many students leveraged positive aspects of their background, for example, valuing humbleness and modesty as a way to manage their own values and maintain social connections (Southgate et al., 2017). At the same time, students often compared themselves to peers and recognized differences including dialect usage (Southgate et al., 2017). A tactic to manage difference includes self-deprecation showing an attempt to create "solidarity with family and community of origin" (Southgate et al. 2017, para. 53).

First-generation students also face unique challenges while adapting to the challenging academic expectations. In a study of transition points of British first-generation students in 
medical school, Bassett et al. (2018) found that many students did not know about common aspects of medical school before starting, including the difference between the common demarcation of basic science and clinical education or the expectation of cadaver dissection, for example (Bassett, 2018). This finding aligns with the argument above that this population of students is not always well positioned to engage informational material. However, there is a paucity of literature related to how first-generation students perform in medical school compared to peers. A Dutch study did find that being first-generation students predicted negative clinical performance more than any other metric (Stegers-Jager et al., 2015). Students with a parent physician, though, was associated with poorer pre-clinical performance, but better performance in the clinical environment (Stegers-Jager et al., 2015). The authors postulate a few theories for why this difference may exist including the likelihood that first-generation students may have less knowledge or comfortability with the clinical environment (Stegers-Jager et al., 2015). Relatedly, there is the concept of cultural bias as students are assessed in the clinical environment, those students perceived to be closely associated with the dominate culture may receive better evaluations (Stegers-Jager, et al., 2015).

First-generation students also face a few additional challenges not necessarily directly related to their academic ability. The literature shows that first-generation students may feel compelled to participate in paid part-time work in an effort to supplement the cost of their education (Bassett et al., 2019; Brosnan et al., 2016). This need to work is partially driven by both the high cost of education and lacking family financial support, but in some instances, the need or expectation to help support their family (Bassett et al., 2019). One of the studies denoted the fluidity of the student's schedule and the need to either find jobs at night or with some type of flexibility, including remote work (Brosnan et al., 2016). Participating in paid-work can also 
have implications for a student's ability to participate in other co- or extra-curriculars including research activity, which can help boost their curriculum vitae (Bassett et al., 2019). As argued by Bassett et al. (2019), how the decision to work ultimately impacts the wellbeing and the ability to persist in medical school is largely understudied.

The literature does show that many first-generation students in medical school struggle to cope with the challenges of the experience and have also indicated a lower quality of life (Mason et al., 2019). First-generation students in medical school have also denoted lower aspects of selfcare, including, for example, engaging healthy nutrition options, getting regular exercise, and implementing techniques to manage stress (Mason et al., 2019). Having role models who are supportive is important for this population for two reasons. First, medical students from firstgeneration families struggle to relate their stress and challenges out of fear and the reality that family may not fully understand (Bassett et al., 2018; Mason et al., 2019). Second, firstgeneration students who intimated greater self-care also indicated that faculty modeled self-care and that the school was attuned to personal challenges, while those with fewer self-care practices did not see faculty as role-modeling those practices (Mason et., 2019). In fact, first-generation students actually worry about engaging faculty members. In one study, students denoted "that they would rather seek the advice of peers than seniors in clinics, because of a fear that their clinical competency would be questioned and help-seeking penalised" (p. 7-8). ${ }^{19}$ One participant stated that it was a common feeling among students that it could be a risk to seek help or guidance from a clinical advisor for fear that any information may not be confidential or could be a detriment to their standing (Bassett et al., 2018).

\footnotetext{
19 The authors do not make clear what the term "senior" is describing. Given that the context of the section where the term "senior" is located, it is presumably in reference to senior physicians as many of the students have positive experiences with advanced standing medical students. (Bassett et al., 2018)
} 
However, as also found in the literature, many first-generation students in medical school are able to leverage some levels of resiliency to succeed (Mason et al., 2019). They also bring a unique perspective to medical school. Several studies have shown that medical students from low-SES or first-generation families may be able to better recognize and relate to patients of similar social backgrounds (Brosnan et al., 2016; Southgate et al., 2017). Examples in the literature include students intimating they felt comfortable using more working-class vernacular or dialect to put the patient at ease (Southgate et al., 2017) as well as a situation where a medical student inquired about medical cost and the patient's ability to pay (Conway-Hicks \& de Groot, 2019). These situations seemed to be as much about the patient as for the student doctor, such as an attempt to connect with the patient and consider aspects of the situation from the patient's perspective but to also maintain identity (Conway-Hicks \& de Groot, 2019; Southgate et al., 2017). In fact, coming from an underprivileged background becomes a part of a medical student's professional identity, and these considerations and perspectives are actually secondnature for this population of doctors (Conway-Hicks \& de Groot, 2019; Southgate et al., 2017).

The literature shows that students begin to integrate into the medical field as soon as they matriculate to medical school. First-generation students arrive with varying comfortability engaging and interacting with practitioners and peers in that new environment. High-SES peers or from families with healthcare providers may be more comfortable with that environment and the transition may be less of a challenge. Students from more privileged backgrounds are likely to have a better understanding of what medical school will entail and have relatable life experiences to their faculty. Evidence shows students from physician families are more likely to perform better in the clinical environment compared to their first-generation students, which may relate to either faculty bias or student comfortability. Although resources exist, first-generation 
students seem to look more to peers for guidance or non-faculty advisors. First-generation students may also experience less obvious challenges such as feeling compelled to work despite a strenuous course load and both emotional and psychological challenges. Such as with firstgeneration students during undergraduate education, many students strike a balance between their home life and their life at medical school despite not always feeling completely at ease within either culture, but caught in the middle, carefully curating their life depending on their audience. Nonetheless, actually the life experiences of first-generation students and low-SES are beneficial in relating to patients and crafting a professional identity.

\section{Conclusions}

The literature shows that first-generation students and low-SES student's decision and ability to attend medical school is influenced by the environment they are exposed to early, including for examples, curricular opportunity, educators, and expectations and guidance from the parent and family. As first-generation students transition to medical school, they tend to arrive with different or less components of the dominate cultural, economic, and social capital valued in the medical school environment. The literature has looked at the experience of firstgeneration students and low-SES students in medical school and has found their experience to include challenges of managing old and new cultural identities while also preserving some of their backgrounds to better engage with patient care. Particularly germane to this proposed research, some literature (Bassett et al., 2019; Brosnan et al., 2016) has examined the experience of first-generation students in medical school through Bourdieu's concepts of capital in Australia and Britain. However, there is a dearth of literature that applies theories such as habitus and capital in examinations of medical students from rural settings and particularly Appalachia, to which this project seeks to begin filling that void. 


\section{Overall Literature Review Conclusions}

Across the four sections of this literature review, there are a number of parallels and conclusions that can be drawn from the research. There are also notable gaps and voids that have yet to be explored. As noted, Appalachia and WV have suffered from economic disparities that are a challenge to ameliorate, deep-seated, and entangled in other ill-structured problems. Educational access and attainment are some of those ill-structured problems. Another is access to modern healthcare and providers, often positioned around population centers. Challenges to obtain higher education and further, medical education, in Appalachia as well as the decision to remain in the region, are compounding and interrelated issues. This literature review sought to align the research and perspectives related to first-generation college and medical students, students from rural and Appalachian backgrounds, and how or why students may be influenced to practice in rural areas.

Foremost, it is clear that first-generation students come to college with different expectations and backgrounds than continuing-generation students. That is in part due to the fact that they are often exposed to differing environments and expectations for their future. Moreover, college presents and promotes individuality and originality, which first-generation students are likely less accustomed to (e.g., Jury, et al, 2017; Orbe, 2004; Roberts \& Rosenwald, 2001; Stephens, Fryberg et al., 2012; Stephens, Townsend et al., 2012). The environment and resultant feelings force a break or conflict with both home culture and family. These findings from the literature draw a clear parallel with rural students, and particularly rural students from first-generation backgrounds. The rural literature, in some respects, has examined both the rural and the rural first-generation students higher education experiences, which is important for this study. Both the first-generation and rural literature provides a foundation for how students from 
Appalachia may experience the medical school environment. Moreover, a small assembly of the literature has delved into the experience of medical students from first-generation or low-SES backgrounds (e.g., Bassett et al., 2019; Brosnan et al., 2016; Mason et al., 2019; Southgate et al., 2017). The majority of these studies have more commonly looked at the experiences of Australian and British medical students (e.g., Bassett et al., 2019; Brosnan et al., 2016; Southgate et al., 2017) and there are fewer examples of literature that has examined the experiences of American medical students (e.g., Mason et al., 2019). These experiences are helpful and beneficial, but their conclusions arrive in different cultural contexts. In some respects, some studies have tied in, tangentially, the experience of seemingly rural students, but rural students have not commonly been an explicit focus in studies of the medical school experience.

The literature has, quite robustly, examined what may motivate a student to practice medicine in a rural area, which does provide some context for the rural medical student experience (e.g., Brooks et al., 2002; Crump et al., 2016; Herd et al., 2016; Stagg et al., 2009). This context includes how they may perceive their rural upbringing and identity. This project does seek to further this line of inquiry. However, I aim to better understand the student perception of their transition and trajectory in light of their Appalachian and rural upbringing as well as their family's educational background and perceptions.

There is no dearth of studies in higher education that have utilized Bourdieu's concepts habitus, capital, field, or others (e.g., Byun, Meece, Irvin, et al., 2012; Beattie \& Thiele, 2016; Patfield et al., 2020; Reay, 1998). Throughout the literature review I have attempted to make note of those utilizations when applicable. The most germane application of those theories, however, were noted in section four when exploring the first-generation or low-SES students' experience in medical school. However, there is a paucity for the application of these theories, 
including habitus, when examining the experience of Appalachian medical students. Moreover, as this literature review has explored the experience of first-generation and rural students in both higher education and medical school, there is a notable absence how Appalachian students, both first-generation and continuing-generation, transition into and through medical school. Recounting the layers of literature surrounding first-generation and rural student experience provides a firm scaffolding to begin considering this medical student population. By applying the literature to this study, it first helps to inform what perspectives and ideas have already been considered and applied to similar questions and populations. Second, this literature review provides a way to compare and contrast the findings of others that have studied related populations or are using Bourdieusian theory.

In sum, the literature informs that the experience of Appalachian medical students is understudied and a scholarship gap. Additionally, the literature shows an ongoing debate between what motivates a student to practice in a rural area. A great deal of that literature has pointed to the role of personal connections and having a rural background. This project seeks to add to that conversation but adding a new lens or angle including how students from a rural background perceive their experience in medical school, particularly in light of the literature that says students, both first-generation and rural, face a challenging disconnect with home during both undergraduate education and medical education. 


\section{Chapter III: Methodology}

With the foundational context of Appalachia as well as a literature review of firstgeneration and rural students, the motivations for students to practice rural medicine, and the experience of first-generation and low-SES medical students, it is prudent to turn to the methods utilized for this research project. Further expounded upon below, this project applied qualitative methodologies of a case-study at a single medical school. It was the intent to recruit seven to ten fourth-year Appalachian medical students for inclusion in the study. A total of ten students were recruited and interviewed through a semi-structured format.

\section{Epistemological Approach}

This study utilizes an interpretative or constructivist approach (Merriam \& Tisdell, 2016). The intent is to understand the experience of Appalachian's attending medical school, and how they perceive practice opportunities moving forward (Merriam \& Tisdell, 2016). This epistemology contends that there is not one viewpoint, truth, or reality, instead all perspectives related to an experience are valid and provide greater understanding and meaning (Merriam \& Tisdell, 2016). As the term constructivist implies creating or forming, this philosophy is the collecting of perspectives to postulate a narrative (Merriam \& Tisdell, 2016). As succinctly stated by Merriam and Tisdell (2016), "Researchers do not 'find' knowledge; they construct it" (p. 9).

\section{Methodology}

This research project follows a single case-study methodology. As informed by Yin (2018), a case-study is appropriate when researching a why or how question. Furthermore, a case-study can be utilized when conditions cannot be applied or situations cannot be controlled or manipulated to answer the questions (Yin, 2018). Yin (2018) argues that a case-study should 
typically meet one of the five expectations, including "critical, unusual, common, revelatory, or longitudinal", to which this project would meet the "common" condition (p. 49). The common rationale is appropriate when "the objective is to capture the circumstances and conditions of an everyday situation - again because of the lessons it might provide about the social processes related to some theoretical interest" (Yin, 2018, p. 50). Appalachians in medical school is not altogether unique in the respect that many students from this region have met the standards to matriculate and successfully complete and practice medicine. However, Appalachian students, particularly those with less fortunate backgrounds or rural backgrounds, may perceive and experience the processes related to medical school differently than peers without those same social backgrounds. Therefore, since there is no need or ability to manipulate the situation and the study fits the common condition, a single case-study methodology is appropriate.

\section{Background and Desired Study Participants}

WVU's School of Medicine has a home campus in Morgantown, WV and two clinical campuses, the Charleston (WV) Campus and the Eastern Campus in Martinsburg, WV. All students complete basic science education (approximately the first two years of the curriculum) in Morgantown. For the clinical phase of the curriculum, $55-60 \%$ of students stay in Morgantown while $25-30 \%$ of students transition to Charleston, and about $10 \%$ of students move to the Eastern Campus. In the third year, students must also complete four weeks at a rural location during the family medicine clerkship and four weeks at a community/rural based rotation during the fourth year of the curriculum. In some instances, certain clerkships or rotations may allow or require students to rotate through a community or rural site for brief periods. 
WVU's medical school is highly selective, ranked by US News \& World Report as the $10^{\text {th }}$ most selective program in the United States (Moody, 2020). In the 2019 admissions cycle, the school received 5,583 applications for only 112 seats in the class to which the school only issued a total of 156 acceptances (2.8\% acceptance rate) (Moody, 2020). In that cycle, only 209 West Virginians applied for admission and 67 of those applicants matriculated. Most entering classes are approximately $60 \%$ in-state residents versus $40 \%$ non-residents. New students typically have high academic metrics, with class undergraduate GPA averages between 3.8 and 3.9 and an average Medical College Admission Test (MCAT) score of 508 ( $74^{\text {th }}$ national percentile) (AAMC, n.d.). Many matriculating students, around 50\%, have an undergraduate degree in the biological sciences (e.g., biology, microbiology, zoology), nearly $30 \%$ have degrees in the physical sciences (e.g., biochemistry, chemistry, engineering), and usually a small percentage have degrees in the social sciences, humanities, or other health professions (e.g., nursing). Around $10-20 \%$ come from double majors or mixed disciplines. Between $50-60 \%$ of medical students are traditional (started immediately after completing their undergraduate degree), meaning that the majority of students begin medical school around the age of 22 years old. The remaining $40-50 \%$ do not start medical school immediately after college. This time is often spent in a number of ways, including, for example, working in a related field to enhance their application, working in an unrelated career, taking a gap year, or serving in the military. Most students start medical school by their mid-twenties, while occasionally a matriculate begins in their 30 s or even 40 s.

For this study, the ideal student was nearing completion of WVU's MD degree program, such as fourth year medical student. Along with the proposed employment of semi-structured interviewing techniques, prospective study participants were asked to complete a questionnaire 
that provides basic demographic or background information (Appendix 1) (Merriam \& Tisdell, 2016). Participants must have spent at least a portion of their formative upbringing in an Appalachian county or counties as identified by the Appalachian Regional Commission (ARC) (Appalachian Regional Commission, n.d.). More specifically, ideal study participants were from West Virginia as the entire state is considered a part of the Appalachian region. Additionally, other areas of consideration were Appalachian counties in Kentucky, North Carolina, Ohio, Pennsylvania, Tennessee, or Virginia. However, students from Appalachian counties in Alabama, Georgia, Mississippi, New York, or South Carolina would not have been considered for participation.

The first survey questions included an inquiry of student's county or counties and state of origin. These questions help identify if a student is from an Appalachian county as defined by the ARC. Moreover, students were asked to relay the amount of time they lived in these counties between birth and the age of 18 . These queries, along with being a fourth year medical student, were the only qualifying questions as only Appalachian students were determined to be considered for the study. However, students may have varying amounts of time living in Appalachia; therefore, it was imperative to know how much time students have lived in the region. Additionally, students were asked to provide their parents' levels of educational completion. This is also an important question, but was not necessarily a qualifying or disqualifying question. As relayed in the first chapter, the Appalachian region suffers from a multitude of educational disparities. The literature review shows that many medical students come from higher earning income families and from families with physicians (Jolly, 2008; Tran et al., 2017). Therefore, it was important to consider both first-generation students and continuing-generation students for this study and consider their Appalachian experience with 
higher education and medical school in relation to the educational background of their family. Having students from both backgrounds in the study helps provide a better understanding of how family educational background may shape or guide the path to medical school among Appalachian students. Moreover, this may present an interesting comparison of experiences. However, it was determined that students of continuing generation status, as defined as one parent who completed at least a four-year degree or parent who is a physician, should not necessarily dominate the study.

Additional questions included basic demographics, such as an age range, time between college and medical school, student's gender, as well as name and location of their undergraduate institution. These questions were not necessarily utilized as qualifying or disqualifying questions for the research study. Instead, they are important to help better understand the data collected and any discernable patterns. For example, age range, time between undergraduate degree and medical school, and undergraduate college location may impact how a student perceives Appalachia and/or medical school. Additionally, as shown in the literature review, students from rural settings may perceive their career options and career trajectory differently based upon gender expectations of their community (e.g., Beasley, 2011; Blackwell \& McLaughlin, 1999; Byun, Meece, Irvin et al., 2012; Dyk \& Wilson, 1999).

\section{The Researcher}

My interest for this research has combined a few different aspects of my background including that I am a first-generation student from rural Appalachia. I completed a master's degree in history with an emphasis in public history and focused a portion of my studies on Appalachian culture and history. I am employed in the WVU School of Medicine's Department of Medical Education. While in the department I have contributed to medical students' 
understanding of Appalachian culture and history by annually presenting an interactive educational session for first-year medical students. The program has been expanded to the WVU Physician Assistant program and the Physician Assistant program at Marshall University.

My first interests into the matters presented in this study surfaced as a result of the aforementioned positions and experiences. Although I have a personal experience in the region, and am vested in better preparing providers for training and practice in Appalachia, these aspects of my background do not bring about particularly unusual biases. Having an interest in the future of a student population as well as having relatable experiences are both expected and manageable. There are also ways to deal with these biases including the utilization of semistructured interviewing, which seeks to present topics but does not attempt to be completely prescribed, such as with structured interviews (Merriam \& Tisdell, 2016). In other words, the interviewee maintains some direction in steering the conversation, although there are designated areas established to be explored (Merriam \& Tisdell, 2016). For data collection, analytical memoing and bracketing, described below, were utilized to manage my own views as the researcher.

I have been provided sufficient guidance and training in preparation for this research project. While completing my master's degree I was able to engage and better understand Appalachian culture and history, which provides a strong fundamental knowledge as well as ability to recognize themes that surface in the data. As a master's degree student, I served as a graduate research assistant for the West Virginia Veterans Legacy Project. In that role I conducted a number of oral history interviews. These interviews provided valuable experiences in qualitative research data collection. Additionally, as a doctoral student I completed two semesters of coursework in qualitative methodology. I also completed courses in the higher 
education program, such as college student development, college student affairs, and diversity issues in higher education, which provided a framework for understanding the theories and literature devoted to the college student experience.

\section{Research Procedures}

Prior to any data collection, the project was submitted to the WVU Institutional Review Board (IRB) and was approved on March 3, 2021 (Appendix 2). After approval, the project was advertised on the email class listserv for medical students in the fourth year. The RedCAP survey was relayed in that email to which several students responded to the survey showing interest. Two students were immediately disqualified because they were not a fourth year student. Over the course of the following weeks, students were contacted and a time was arranged to meet, creating a purposeful, convenience sample for the study (Merriam \& Tisdell, 2016). Interviews with students were conducted via the Zoom video conferencing software and recorded. Students were commonly asked at the end of the interview if they could either relay or alert other students of the project for potential participation. In at least one instance a student's name was relayed to researcher who reached out to the student who ultimately agreed to participate. The researcher did not reach out to all students who were suggested in part wanting to keep the participant number around ten and because in the latter phases of recruitment the researcher was seeking first-generation students, ensuring gender-balance, and strongly considering rural upbringing. In an effort to increase first-generation student participation and students from rural areas, some students known or believed to fit those categories were emailed separately from the listserv asking them to consider be a part of the project. At a later time, several of those students were sent a second follow-up, to which one additional student responded to participate, which was the final student included in the study. 
Prior to beginning the interview, informed consent was verbally relayed through a cover letter. This study did not include minors or vulnerable populations; therefore, a signed informed consent was not utilized (see Appendix 3 for cover letter). The verbally relayed informed consent letter was also emailed to the student. The researcher was the only individual who conducted student interviews. Students were notified that the interview was being recorded for the researcher's reference. They were alerted that they may skip any question or discontinue at any time and there would be no negative implications, such as impacts to class standing by doing so. They were also notified that the interview would take up to about one hour and they would receive one \$10 Amazon gift-card for their participation. The gift card was funded by the researcher. The student was also made aware that collected and reported data would be anonymous and care would be taken to protect their identity. All subject names referenced in this study are pseudonyms selected by the researcher. Specific references to place names, including hometowns and high schools, were changed or obscured to add extra protections. Original unedited interviews and transcripts were saved to a secure sever requiring a password and dual authentication. Maintaining transcripts will be beneficial for upholding validity and reliability (Merriam \& Tisdell, 2016). A pseudonym key was recorded in a paperback notebook away from the original interviews and transcripts. Deidentified transcripts and memos were saved in a separate location away from the aforementioned location.

\section{Data Collection}

Data collection began with the aforenoted pre-survey. Questionnaire data was collected and managed using REDCap (Research Electronic Data Capture) hosted at WVU (Harris et al., 2009; Harris et al., 2019). REDCap is a secure, web-based software platform designed to support data capture for research studies (Harris et al., 2009; Harris et al., 2019). The provided 
information serves two purposes, including originally helping to determine if a student would be included or excluded from the study participants and to collect some uniform data. Interviews followed a semi-structured interview format with the romantic philosophy. The purpose of using a semi-structured interview allowed some specific questions to be covered, but not overly calculated, giving the respondent the opportunity to expound on certain areas (Merriam \& Tisdell, 2016). The romantic interview is important because it helps establish rapport with the subject and allow for a conversation between the interviewer and the interviewee (Merriam \& Tisdell, 2016). Interviews typically lasted between 40-50 minutes, and none went more than one hour.

The interviews began by asking about perceptions and relationship with Appalachia to allow the respondent to begin reflecting on the region and associations (see Appendix 4 for protocol). This line of questioning helped the respondent begin considering the Appalachian context. From there, questioning moved to that actual experience of leaving for college and starting medical school. This line of inquiry segued into discussing relevant tensions, if there were any, that the student may have perceived between college/medical school and home (i.e., Appalachia) as well as what motived them to attend medical school. Ultimately, interviews typically concluded with a discussion of what may or may not be motiving their future practice plans, including where they may consider practicing.

\section{Data Analysis, Validity, and Reliability}

The interviews were auto-transcribed through the transcription service incorporated within the Zoom videoconference software. However, not all of the interviews were transcribed accurately, which related to sound quality, dialect, or language used, as well as general software inaccuracy. Therefore, the researcher read the transcriptions making obvious corrections and 
then listened to the interviews in entirety while reviewing the transcript to ensure accuracy. Transcriptions were completed in the order of the interviews. After each interview, the researcher crafted an analytical memo (Merriam \& Tillman, 2016). Memos captured initial perceptions that surfaced during the interview, included general observations of the interviewee, emergent themes, and overall impressions that could help guide aspects of subsequent interviews (Merriam \& Tillman, 2016). Of note, memoing was helpful for drawing connections to interviews that had already occurred and emergent or possible themes that were surfacing throughout the course of the interview. The researcher also utilized bracketing to be sure to capture thoughts that may be rooted in biases, particularly as an Appalachian (Merriam \& Tillman, 2016).

Memoing and analysis of interview transcripts utilized an inductive approach. First, since interviews were transcribed within the order they were conducted and during the interviewing process, the researcher undertook a constant comparative analysis (Merriam \& Tisdell, 2016). This analysis involved repeatedly returning to previous transcripts and memos and considering differences and similarities with new interviews. This further helped inform analysis, particularly open-coding (Merrian \& Tisdell, 2016). The researcher utilized NVivo software to open-code transcripts. Codes were applied in essentially two rounds. The bulk of the coding occurred with the first round of coding. During the second round of coding significantly fewer codes were applied. Along with open coding, analytical memos were referenced to be sure that identified themes were considered and ascertaining if they were still applicable when considering the totality of the interviews. After analyzing the data in NVivo, the researcher began organizing and categorizing themes and data for reporting. In part, the process also involved returning to the literature review and determining connections, similarities, and incongruities to others' findings. 
Otherwise, all codes were considered. Some codes were considered duplicative or imprecise. In those instances, codes were often combined with other codes or reworded for more precision (Merriam \& Tisdell, 2016). Many initial codes were reorganized and characterized as subcodes. Subcodes were organized with overarching or categorical codes (Merriam \& Tisdell, 2016). Codes and subcodes are identified in Table 1. 
Table 1

\begin{tabular}{|c|c|}
\hline Categorical Code & Sub-Code \\
\hline Appalachia Meaning & Community; Culture; Geography; Hardworking or blue collar; Home; Nature; Rural; Sense of place \\
\hline $\begin{array}{l}\text { Appalachian } \\
\text { Challenges }\end{array}$ & $\begin{array}{l}\text { Big industry; Brain drain; Broadband; Despair; Food deserts; Limited healthcare access; Loss of industry; Opioid misuse- } \\
\text { drug usage; Poor health outcomes; Provider shortage; Socioeconomic challenges }\end{array}$ \\
\hline Appalachian Identity & $\begin{array}{l}\text { Appalachian generational association; Appalachian stereotypes; Connection to a physical place; Identify as Appalachian; } \\
\text { Identify as West Virginia; Less than } 100 \% \text { ID; Non-identification }\end{array}$ \\
\hline $\begin{array}{l}\text { Appalachian Patient } \\
\text { Characteristics }\end{array}$ & Patient distrust; Waiting for care \\
\hline $\begin{array}{l}\text { Barrier to Stay- } \\
\text { Motivation to Leave }\end{array}$ & $\begin{array}{l}\text { Barrier family opportunity; Barrier isolation; Barrier socialization; Barrier to stay specialization or sub-specialization; Group } \\
\text { practice; Lack of diversity; Leave \& come back, Telemedicine, Outreach; Limited resources ;Motivation to leave amenities; } \\
\text { Motivation to leave weather; No rural desire; Out of state students; Physicians motivating to leave; Work in academia; } \\
\text { Work-life balance }\end{array}$ \\
\hline College Prep Courses & College prep. could have done better or more; Fewer college prep. courses than others \\
\hline College Transition & $\begin{array}{l}\text { College and family ties; College proximity, commuting; College Support-No or limited dissuasion; College wakeup call; } \\
\text { Dad educational influence; Dad indifferent; Different gender expectations; Easy college transition; Extended family college } \\
\text { transition; Mom educational influence; Non family influence for college; Parents support for college; Went home a lot }\end{array}$ \\
\hline Decide College & Always college \\
\hline Decide Medicine & $\begin{array}{l}\text { Decide medicine in college; Decide medicine in high school; Decide medicine in middle school; Decide medicine at young } \\
\text { age }\end{array}$ \\
\hline $\begin{array}{l}\text { Desire rural practice or } \\
\text { living }\end{array}$ & Desire rural WV practice; Live rural but work elsewhere; Living rural preference \\
\hline $\begin{array}{l}\text { Finances, Jobs, Cost of } \\
\text { Living }\end{array}$ & Cost of living; General job or financial motivations; Housing market; Rural job availability \\
\hline M3 Rural Rotation & $\begin{array}{l}\text { M3 different practice model; M3 rural do not identify with population; M3 rural experience shows more limited resources; } \\
\text { M3 rural eye opening; M3 rural Identify with patients; M3 rural important for out of state students; M3 rural interactions } \\
\text { important; M3 rural negative; M3 rural positive; Cities/University different }\end{array}$ \\
\hline
\end{tabular}




\section{Table 1}

Categorical Code Sub-Code

M4 Community

Rotation Experience

Medicine Influence

Metrics

Motivation to stay or not a barrier

Pre-College Education and Opportunities

Specialty Choice

Student characteristics

Uncertainty or other considerations for practice

Upbringing
M4 community experience interactions with patients; M4 community experience positive; M4 community experience eye opening; M4 rural with limited resources

Exposed to sciences; Family pushback or questioning about medicine; Family support for medicine; Good at science; Influential professor; Limited medical influence; Medical influence; Medicine questioning; Medicine mental challenge; Medicine TV influence; Negative or dissuading physicians; No one to tell you how hard medical school is; Pediatrician positive; Positive physician influence; Positive shadowing

High achievement motivation to leave; Metrics determine specialty choice for some; Metrics not influential for practice location; Metrics tension

Indifferent about amenities like restaurants, etc.; Motivation home and family; Motivation to stay cheaper private education; Motivation to stay outdoor activity; Motivation to stay to serve state, region, people; New resources \& More connected world; Opportunity for practice not limited in rural area; Positive rural physician influence; Positive to be rooted in the community; Rural opportunities for leadership; Specialization as a factor

Negative educational experience; Positive educational experience; Private education; Saw other opportunities elsewhere

Emergency medicine; Family medicine; Internal medicine; OBGYN; Pediatrics; Psychiatry

First-generation college student; Internal locus of control; Previous career; Self-motivation

Influence of partner; Mixed messaging on staying versus leaving; No desire to subspecialize; Residency program fit; Unsure about future practice location

Close extended family; Close immediate family; Community friends; Education valued in family; Fortunate upbringing; Limited things to do; Non-rural upbringing; Rural upbringing; School consolidation; School diversity; Sibling trajectory; Small school; Socioeconomic influences for education; Sports; Travel-family vacations 
Several strategies were utilized to achieve validity and reliability including, "adequate engagement in data collection" (Merriam \& Tisdell, 2016, p. 246). In part, this includes working to ensure that enough data is collected to answer the research questions. The researcher achieved this end by seeking data saturation, or reaching a point where it is unlikely that more interviews would add to the themes already present in the collected narratives (Merriam \& Tisdell, 2016). Adequate data engagement was also achieved through open coding all transcripts. Additionally, memoing also helped achieve reliability and validity by creating an audit trail of both documentation, which is important for validity, but also in the researchers own thinking, which may have changed through the research process. Lastly, reliability and validity were achieved through the variability of the research subjects (Merriam \& Tisdell, 2016). As noted, each student's background was considered before each interview.

\section{Student Sample}

The sample includes six female students and four male student (Table 2). Seven of the students denoted being born in West Virginia. Of the three not born in West Virginia, one moved to the state at a young age from an Appalachian Maryland county. One student recounted moving to West Virginia at age ten from a major American city, but has since lived in the state including for college. Finally, the remaining student was born and raised in southwestern Pennsylvania, in an Appalachian county. It is difficult to ultimately determine if a student grew up in a rural locality. There is no standard definition of what accounts for being rural, and in some respect rural is a subjective concept. However, in an attempt to quantify how many students indeed grew up rurally, 2010 US Census data were utilized along with given definitions. The US Census Bureau (2019) does not define the term rural but instead rural includes every area not defined as an "urbanized area" or an "urban cluster." Urbanized areas include more than 50,000 people, of 
which there are nine that include at least parts of West Virginia (US Census Bureau, 2019).

There are 41 urban clusters within or partially within West Virginia, which are towns or areas with between 2,500 and 50,000 people (US Census Bureau, 2019). The largest urban area is Charleston, West Virginia with approximately 153,000 people and the second is Huntington with approximately 112,000 people (which includes parts of Kentucky and Ohio) (US Census Bureau, 2019). No other urban area that include a part or all of a West Virginia town or city has more than 100,000 people(US Census Bureau, 2019).

By using the US Census Bureau definition of urbanized areas and urban clusters, five of the students in this study technically grew up in urban areas and three grew up in urban clusters, while two grew up in rural areas. However, there are difficulties with using this definition and in fact, students in this study had quite a bit more rural background than this definition allows. First, these definitions are arbitrary in that they have rigid cut offs. For one of the students, their town had fewer than 200 more people than the 2,500 bottom threshold for being an urban cluster. One of the students who grew up in an urban location also had a second family home in a rural area of that the state that they were attached to and that home were discussed as cultivating a strong connection to the state. Another student's hometown had around 1,000 people and was about 20 miles away from an urban area; however, the US Census Bureau includes that town of 1,000 people in the greater urban area.

A slightly different way to consider if a student grew up in a rural background would be to explore the classification of their high school's school system. For the nine West Virginia students, their school system would essentially be their home county. The Universal Services Administrative Company (USAC, 2021b) utilizes census data to ascertain if a school system is 
located in an urban or rural area. ${ }^{20}$ When $50 \%$ or fewer of all schools in a school district are considered urban, than the USAC (2021b) characterizes the district as rural; otherwise, the school system is considered urban. By this methodology, five students' school system fall into the urban category and five fall into the rural category. For the two students who attended private school, they were counted within the school system that corresponded to the geographical location of their private school, although the private school was technically not in the district. Four of the students originally characterized as growing up in an urban community actually attended a rural school system. However, for one of the private school students, they grew up rurally but commuted to an urban area/school district instead of attending the local high school. Therefore, the original hometown classification put eight students growing up in urban areas/clusters and two growing up rurally; however, five students were from predominantly urban school systems and five students were from predominantly rural school systems. The difference between ruralness of hometown and school district actually switched for five of the students.

Several points need to be made to better understand this sample and their "ruralness". It is possible to characterize students by where they grew up as either urban, an urban cluster, or by default rural as characterized by the US Census Bureau (2019). However, these categories are somewhat arbitrary. As already noted, there is a subjectivity to the term rural. Moreover, for five students, where they grew up and their school system makeup had different categorizations. This

\footnotetext{
20 The Universal Service Administrative Company (USAC, 2021a) is a non-profit organization that allocates funds from telecommunications companies to expand and enhance connectivity (e.g., broadband, telephone services, etc.). All telecommunications companies are mandated to contribute funds as delineated by the Telecommunications Act of 1996 (USAC, 2021a). The USAC has a particular interest in expanding and enhancing telecommunications capabilities to rural and underserved areas. The USAC provides a database that relies on the US Census Bureau to determine if certain entities fall within either urban areas (including urban clusters) or rural areas, to ascertain if certain services or discounts for services are applicable (USAC, 2021b). Those entities include, but are not limited to, schools and school systems, which are germane to this study (USAC, 2021b).
} 
difference shows how proximal, geographically, rural and non-rural areas are in West Virginia.

That is to say that students in this study may have grown up in a rural area but their school district was mostly in an urban area or conversely, they grew up in a more urban area, but the majority of their school system was rural. The students from this study, save one from Pennsylvania, are all from West Virginia, a relatively small state in both population and area. Only two urban areas have populations greater than 100,000 people and one of those technically includes parts of two other states. The argument is that it is possible, by US Census Bureau data or otherwise, to characterize students as either urban or not, but for even the students who came from categorized urban areas, these are not major American cities. Appalachia is a rural region and this student sample represents Appalachia in that it includes students from both more urbanized and rural areas. The ten students in this study hail from eight different West Virginia counties and one Pennsylvania county. They are representational of the region inclusive of students from rural area, small towns, and more urbanized small cities.

Table 2

Demographic of Student Sample

\begin{tabular}{|c|c|c|c|c|c|c|c|}
\hline Student* & Upbringing & $\begin{array}{l}\text { Age } \\
\text { Range }\end{array}$ & $\begin{array}{l}\text { Parent's Education } \\
\text { (Father/Mother) }\end{array}$ & $\begin{array}{l}\text { High } \\
\text { School }\end{array}$ & $\begin{array}{l}\text { School } \\
\text { District }\end{array}$ & $\begin{array}{l}\text { Residency } \\
\text { Specialty }\end{array}$ & $\begin{array}{l}\text { Post-residency } \\
\text { Practice }\end{array}$ \\
\hline Mark & $\begin{array}{l}\text { WV, South } \\
\text { Central }\end{array}$ & $23-26$ & $\begin{array}{l}\text { Post-secondary } \\
\text { training/Four Yr. } \\
\text { Degree }\end{array}$ & $\begin{array}{l}\text { Public, } \\
\text { Low- } \\
\text { Income }\end{array}$ & Urban & OBGYN & Rural \\
\hline Megan` $^{\wedge}$ & $\begin{array}{l}\text { WV, } \\
\text { Northern } \\
\text { Panhandle }\end{array}$ & $23-26$ & $\begin{array}{l}\text { Diploma/Post- } \\
\text { secondary education }\end{array}$ & $\begin{array}{l}\text { Public, } \\
\text { Low- } \\
\text { Income }\end{array}$ & Rural & OBGYN & Undecided \\
\hline Jared & $\begin{array}{l}\text { WV, } \\
\text { Eastern } \\
\text { Panhandle }\end{array}$ & $23-26$ & $\begin{array}{l}\text { Both, Four Yr. } \\
\text { Degree }\end{array}$ & Public & Rural & OBGYN & Non-rural \\
\hline Emma & $\begin{array}{l}\text { WV, North } \\
\text { Central }\end{array}$ & $23-26$ & $\begin{array}{l}\text { Both, Four Yr. } \\
\text { Degree }\end{array}$ & Public & Urban & Psychiatry & Undecided \\
\hline Steve ${ }^{\wedge}$ & $\begin{array}{l}\text { PA, } \\
\text { Southwest }\end{array}$ & $23-26$ & $\begin{array}{l}\text { Diploma/Post- } \\
\text { secondary education } \\
\text { (Associates) }\end{array}$ & Private & Urban & $\begin{array}{l}\text { Emergency } \\
\text { Medicine }\end{array}$ & Undecided \\
\hline
\end{tabular}


Table 2

Demographic of Student Sample

\begin{tabular}{|c|c|c|c|c|c|c|c|}
\hline Student* & Upbringing & $\begin{array}{l}\text { Age } \\
\text { Range }\end{array}$ & $\begin{array}{l}\text { Parent's Education } \\
\text { (Father/Mother) }\end{array}$ & $\begin{array}{l}\text { High } \\
\text { School }\end{array}$ & $\begin{array}{l}\text { School } \\
\text { District }\end{array}$ & $\begin{array}{l}\text { Residency } \\
\text { Specialty }\end{array}$ & $\begin{array}{l}\text { Post-residency } \\
\text { Practice }\end{array}$ \\
\hline Dianne $^{\wedge}$ & $\begin{array}{l}\text { WV, Ohio } \\
\text { River } \\
\text { Valley }\end{array}$ & 31 or $>$ & $\begin{array}{l}\text { Some college/ } \\
\text { Diploma }\end{array}$ & $\begin{array}{l}\text { Public, } \\
\text { Low- } \\
\text { Income }\end{array}$ & Rural & $\begin{array}{l}\text { Internal } \\
\text { Medicine }\end{array}$ & Rural \\
\hline Hannah & $\begin{array}{l}\text { WV, South } \\
\text { Central }\end{array}$ & 31 or $>$ & Diploma/Doctorate & Private & Urban & $\begin{array}{l}\text { Family } \\
\text { Medicine }\end{array}$ & Rural \\
\hline Leah & $\begin{array}{l}\text { WV, } \\
\text { Eastern } \\
\text { Panhandle }\end{array}$ & $23-26$ & $\begin{array}{l}\text { Both, Master's } \\
\text { Degree }\end{array}$ & $\begin{array}{l}\text { Public, } \\
\text { Low- } \\
\text { Income }\end{array}$ & Rural & Psychiatry & Undecided \\
\hline Heidi & $\begin{array}{l}\text { WV, } \\
\text { Southern }\end{array}$ & $23-26$ & $\begin{array}{l}\text { Master's Degree/Four } \\
\text { Yr. Degree }\end{array}$ & $\begin{array}{l}\text { Public, } \\
\text { Low- } \\
\text { Income }\end{array}$ & Urban & Pediatrics & Non-Rural \\
\hline Isaac $^{\wedge}$ & $\begin{array}{l}\text { WV, North } \\
\text { Central }\end{array}$ & $23-26$ & $\begin{array}{l}\text { Some college/ } \\
\text { Diploma }\end{array}$ & $\begin{array}{l}\text { Public, } \\
\text { Low- } \\
\text { Income }\end{array}$ & Rural & $\begin{array}{l}\text { Family } \\
\text { Medicine }\end{array}$ & Rural \\
\hline
\end{tabular}

*Pseudonym

$\wedge^{\wedge}$ First-generation student

Among the sample, four students attended college as first-generation students. An original aim of this study was to have at least a comparable balance between first-generation and continuing generation students. Moreover, continuing generation students could not dominate the study. Although it is not precisely equal, there is representation by first-generation students and there is a mix of family educational backgrounds. It is challenging to recruit first-generation students, notably because each class of medical students at this institution is composed of approximately $10 \%$ first-generation students. Of the first-generation students, Dianne and Isaac each had a parent that attended college but did not graduate. Neither of Megan's parents attended college, but her mother did complete nursing training. Of Steve's parents, his mother completed an associate degree. Of the remaining parents of the first-generation students, all achieved a high school diploma. Aside from first-generation students, the sample had a mix of parental education. There were also a mix of other levels of educational attainment including some with one parent who completed college and one parent who completed high school as well as dual 
achievement of college degrees to various levels. Notably, Hannah's mother completed a terminal degree (the only student in the study with a parent who graduated from a doctoral program), while her father completed a high school diploma. In sum, a potential limitation to this study is the number of first-generation students; however, the remaining six non-first generation students represent a wide variety of parent educational achievement from high school diploma to doctoral training.

Aside from identification as rural and parent educational level, there are a few other notable characteristics of the sample. First, six students attended high schools that are considered to be low-income. ${ }^{21}$ Two other students attended private Catholic high schools. Most students were between the ages of 23-26 at the time of the interview, which is somewhat typical. However, two students who had spent time working in other careers outside of West Virginia and were older. Four of the ten students attended WVU for undergraduate education, while six graduated from other institutions. Four of the students will be in a primary care specialty for residency, including family medicine, internal medicine, and pediatrics. Two students will be psychiatrists; one student is pursuing a career in emergency medicine; and, three students will be obstetricians and gynecologists. Four of the ten students denoted a desire to practice rurally, although not necessarily in Appalachia. Four were undecided if they would practice rurally. Two denoted little or no desire to practice in a rural location. As is recounted in Chapter IV, students have varying levels of Appalachian and West Virginian identity. For the most part, other identities were not necessarily asked about. However, one student did offer that they also

\footnotetext{
21 The Federal Student Aid: An Office of the US Department of Education Teacher Cancellation Low Income database was utilized to determine if the student's high school was considered low income by the federal government. Schools in the database and recognized as low-income are eligible for Title One funds under the Elementary and Secondary Education Act and 30\% of students at the specific school are eligible for Title One aid and services. (Federal Student Aid: An Office of the US Department of Education, nda\&b).
} 
identified as Puerto Rican. One other student denoted identification as lesbian, gay, bisexual, transgender, or queer (LGBTQ). In sum, this sample of medical students covers a broad range of geographical locations as well as educational background and family educational background. The sample does not include an exact balance of males to females, but is comparable. Moreover, students have a broad range of residency and practice plans from primary care to surgical specialties. Students are at various levels of certainty on if they plan to practice rurally.

\section{Ethical Considerations}

As previously noted, this research project was reviewed and approved by WVU's IRB. There is a shared learning experience between the researcher and the subject and, as told by Merriam and Tisdell (2016, p. 262) in referencing and quoting Patton (2015, p. 494), "the interviewer is neither a judge nor a therapist nor 'a cold slab of granite..."' It is incumbent on the researcher to be prepared to guide participants to applicable support and resources, if warranted (Merriam \& Tisdell, 2016). The intent of this research was not to explore or recount traumatic or stressful situations in a participant's past. As a part of qualitative research, participating in a research project may benefit the subject by exploring and learning about their self and ways of thinking (Merriam \& Tisdell, 2016). The purpose of this project was, in part, to learn more about the transition to and through medical school for Appalachians. There was no intent that any of the interview questions would seek out any traumatic or challenging past experiences, but the remote possibility did exist considering that the literature shows some students hold on to and leverage childhood experiences (e.g., educator, family, or social dissuasion) for motivation and resilience in higher education and medical school (e.g., Bassett et al., 2018; O'Shea, 2016; Tate et al., 2015; Towers, 2005; Welch, 2013). To the researcher's knowledge, no students felt or appeared to be significantly or emotionally impacted by the interview. 
As already explained, students were verbally relayed the informed consent before each interview began. Student were informed that their responses would remain anonymous. The researcher was the only individual to work with the raw interview transcripts and data. No medical school deans or course directors were or will have access to identifiable interview recordings or transcripts. Any and all quotes that are used are deidentified with pseudonyms. The recordings and raw transcripts of the interviews have been stored on a secure password protected server that require dual authentication and away from deidentified transcripts. Finally, no perceived conflict of interests were identified with any research participants. The researcher is not currently serving in any capacity to evaluate any students in this study. The researcher is also not related to any current medical students at WVU nor does he have a personal relationship with any students involved in the research subject.

\section{Summary}

This project is a case-study of Appalachian medical students at a single institution. The general aim of the project is to better understand how students perceive their experience in getting to medical school, how they recognize and understand their current status in medical school, and how their background may guide their plans for future practice location. The project

will assume a constructivist/interpretivist epistemology. Before starting the project IRB approval was sought and received. For data collection, ten semi-structured interviews of fourth-year medical students allowed for data saturation. Interviewees only included students from Appalachia, and incorporated both first-generation and continuing-generation students, with the initial aim to not have disproportionately greater numbers of continuing-generation students. This sample included four first-generation students and six non-first-generation students. Recruitment of subjects occurred through a purposeful, convenience sampling. Data collection 
utilized a case study approach with analytical memoing and constant comparative analysis to aid validity. Bracketing helped manage researcher biases. Primary analysis of transcripts included open coding and utilization of NVivo software. In relaying findings, subjects were deidentified and given pseudonyms 


\section{Chapter IV: Upbringing and Schooling}

\section{Research Question I: How do Appalachian students interpret their relationship with home - including cultural associations - as they transition through the medical education program?}

At the start of each interview, participants were asked about their demographic background including where the student grew up, information about the schools they attended, where they went to college, their college major and questions about family, including parent's educational attainment. From there, students were asked to describe what the word "Appalachia" meant to them with a follow-up probing question that asked how much or in what ways the student identified as Appalachian. This conversation was important to begin understanding cultural associations and affiliations students may have with the region. All of the students, with the exception of Jared, denoted that they identified as either being Appalachian, some aspect of Appalachian culture, or as a West Virginian. Jared explained:

The idea of... Appalachian culture doesn't really come to my mind, that's not really something... I understand so much. I think that might have to partly... be because... my county is right on the border of Maryland, so we kind of have a different perspective of like West Virginians than maybe people here in Charleston... do. To me it's just like a geographical... landscape.

Some students drew a more nuanced difference between identifying as Appalachian and West Virginian. Moreover, some made a similar point as to where in West Virginia may be considered Appalachian versus not Appalachian. Dianne was asked about her identification with the region and she explained that she saw herself as a West Virginian and described her formative community on the Ohio River and its similarities to Pittsburgh. "But when I look back 
I'm like, yeah I did grow up... rural, but when I think of Appalachia, I think more of like where I'm at in Charleston, like southern West Virginia. The... people living in the hollers. We had that too... there were plenty of people from the hollers." Similarly, Hannah, who grew up in rural, south central West Virginia explained that she felt a generational attachment, citing the possibility of her ancestors' involvement in the Civil War. She further explained, “And I feel connected to West Virginia, in a way that I think other people aren't connected to their states and... I don't know if that's like a West Virginia thing or Appalachian thing...”

Hannah's comment about her ancestors' participation in the American Civil War points to a common narrative that surfaced in many of the interviews. Students commonly positioned themselves as Appalachian in relation to their known ancestry and the volume of family living in the region, particularly in their home community or county. Emma noted, "My dad's side of the family... is one of... the earliest families to... settle in the area where I grew up... like the [last] name is on the... courthouse. So, I identify strong with being Appalachian.” Dianne noted, "my family is umpteen generation West Virginian, I mean as far back as I can go everybody's from West Virginia... which I think is most people in West Virginia.” Some students, including Hannah, Steve, and Isaac recounted the proximity of their extended family growing up, which included a farm or grandparents as a central focal point to their upbringing. Steve, who talked proudly of his family's southwestern Pennsylvania dairy farm explained:

The first neighbor, which is a quarter mile away, he's like third cousin or something you know, they're related and stuff... then the next one is my mom's original farm and that's my... third or fourth cousin who lives there and then after that would have been my grandma's house, which is where my sister is living now because she's... taken over the farm. 
Conversely, but to solidify this point, Leah who moved to the Eastern Panhandle from a major city at age ten claimed that she did consider herself Appalachian now after staying in West Virginia for both college and medical school. However, when asked how much she identified as Appalachian, she responded by saying:

I would probably say maybe like $75 \%$, that's probably a strange percentage but... I know I wasn't born here and... my family's not from here... I know a lot of people identify Appalachia as like their... whole lineage lived here, or like their family were like coal miners or like farmers.

As a follow up, she was asked if she may feel differently if her parents or grandparents grew up in Appalachia, to which she interjected that her grandmother was from West Virginia and stated, "she knew the Hatfields and McCoys, she like knew them..." Later she said:

I definitely think that I almost felt like an imposter for a long time, because... my family wasn't from the state... I didn't necessarily have all of the struggles that I think children maybe who... come through multiple generations of individuals living here might face. Leah was not necessarily an Appalachian by birthright but by self-identification with time and milestones in the region still relied on the idea of generational establishment to help determine that association. She is unique in the fact that she spent the first ten years of her life elsewhere, the only individual in the study with that experience. However, she relied on her generational associations, a grandparent that knew the Hatfields and McCoys, recognizable figures in Appalachia, but also judged that she did not have enough lineage to be fully Appalachian.

Although Leah's background is unique in this study, several students also quantified or qualified their Appalachian identity. Mark, who grew up in a more populated area of central West Virginia, explained: 
I don't want to say $100 \%$, I don't really know how else... how to quantify it... Identifying as Appalachian can... mean different things for different people... I don't always necessarily identify with some of the more... generalized aspects of Appalachian culture, you know I didn't... really grow up in... a rural area.

He further noted his upbringing in a populous area as an example of how he is more "at a distance" from full identification. Isaac from a rural community in northern West Virginia made several references to the family farm, but also denoted a less than $100 \%$ identification as being Appalachian. When asked how much he identified, he explained:

You know, less than my friends... I think, because of, I was more open to different opportunities... a couple of my buddies that went to [college] with me, moved back... and they ride around on their side-by-sides down to... the [local bridge]... that's kind of every weekend,... so I'm not as, compared to them. I would say, maybe $80,80 \%, 85 \% .^{22}$

His response is similar, in some respects to Steve, who denoted:

I see myself as Appalachian in my personality and in my... characteristics... I'll give somebody a hand if they need a hand... I'll do it myself if... I can... but then... there's also a sense of Appalachia where... people think that it's like... hunting, fishing, wearing camo. I don't identify with that, but I identify with the characteristics of community and kind of helping each other out.

The comments above are illustrative of the nuances in the responses that were given. Jared did not identify as Appalachian, but only one student offered up a competing identification to being Appalachian or West Virginia. Heidi referenced, like others, the values of hard work, being family oriented, and similar values, but did not as closely identify with the music or food

\footnotetext{
${ }^{22}$ Side-by-side are an all-terrain off-road vehicle similar to a four-wheeler.
} 
of the region. However, she also explicated that one set of grandparents were from Puerto Rico and she had spent periods of time with them growing up, including making trips to the island. Heidi, as well as many other students, made note of aspects of being Appalachian they do not necessarily identify with, but she is the only student to explain a separate cultural identityPuerto Rican.

To summarize, many students based their identification with Appalachia or West Virginia along the lines of generational associations. Often, students referenced the amount of time family had been in the region and how that may contribute to their identity. Some more closely identified with being West Virginian as opposed to necessarily Appalachian, while others explained that they identified with some aspects of Appalachia or being Appalachian, but added a qualifier. For example, some qualifiers included having a weak generational association, not partaking in certain activities, or not feeling that they had enough of a truly rural background. There were also subtle notions of avoiding or not partaking in certain stereotypical activities (e.g., riding side-by-sides, hunting, fishing, etc.). Although generational ties were positive and were seen as a way to draw a longstanding connection to the state or region, certain stereotypes, and not participating in those activities were a way to separate oneself from full identification. Considering these nuances in how this sample identified with Appalachia and being Appalachian is vital for understanding the themes associated with this research question.

\section{Theme 1: Family, Community, \& Land Associations Remain Important}

Family Dynamics: During the interviews, students were asked about their transition to college and undergraduate education and being away from home and family, including how that may or may not have impacted relationships with family members. As these were fourth year medical students, this transition began at least eight years prior, and longer ago for some. Mark, 
Megan, Emma, Jared, and Dianne did not recognize any major changes with relationships at home when first going to college. Mark, who went to a major university in an adjacent state described an easy transition, but still stated, "It was definitely... a lot... to take in, I think, just like for any freshman going to a larger school." Megan, a first-generation student, chalked up a smoother transition to only being an hour and a half away and stated, "I think if I... would have went further, I think it might have been more of an issue" and followed up by saying if she had gone further away, her parents, “would have wanted me closer to home.” Dianne, also a firstgeneration student, went to college immediately after high school, but held a different career before medical school. She was "ready to break out of the small town and get into the big world" but claimed her college town "wasn't exactly huge" and only about a 45-minute drive from home. She mentioned that she had always been close to her parents and college did not change her relationships. Although she did explain some academic challenges in math and science, she did not indicate struggles with being away. Jared, from West Virginia's Eastern Panhandle, also expressed an easy college transition. He explained, “...for the first week... there's an adjustment period I think everyone experiences. It's... a universal college experience. But, dude, the moment... I went to my first college party and... made friends... it was game over."

Conversely, the remaining students described changing family dynamics including some who worried about leaving and others who ultimately felt a strengthened relationship. Steve, a first-generation student who attended a major university outside of West Virginia, denoted:

I think, maybe, [college] strengthened [my relationship with back home and family] because I... realized how much I... loved being on the farm and just around my family... I think it strengthened my desire to stay at home and keep living that rural lifestyle. 
Although Steve did not indicate being particularly upset or having emotional struggles with being away, Hannah and Isaac did. Hannah explained that she was particularly close with her grandparents who lived next door. She described her grandfather's declining health while she was leaving for college and noted, "I felt and still feel a strong responsibility towards my grandparents... and taking care of them, because that's what people in Appalachia do right, like you take care of your family." She ultimately went to a private college outside of West Virginia and denoted frequent calls and trips home.

Isaac, also a first-generation student who commuted his first year of college, explained a challenging transition when he moved on campus his sophomore year. He described going home a lot of weekends and then having a hard time going back to college. "When I would leave to go back... I'd be just distraught. I'd be upset... Even though... things were great at [college]." He also explained that he got a lot closer with his parents while in college. "The further I got away... made our time more valuable when we did get back together.” Although Leah and Heidi did not indicate that they struggled with being away from parents and family, they did convey a strengthened family dynamic and relationships, much like Isaac and Steve. Heidi explained that her family also moved away from southern West Virginia the same summer she left for college, so she lost a lot of her connection with her hometown and rarely returned. Instead, she visited her parent's new community, also in West Virginia, and since she did not know anyone there, she intimated more quality time on trips home and generally closer relationships. Leah also signified a stronger relationship with her family and stated that her college, four hours away from home, introduced her to different family dynamics and family backgrounds. "It made me appreciate... how much my family loved me and that I had a safe home to go home to.” 
For the students who described struggles or a changing dynamic with parents, Isaac and Hannah recounted a difficulty with leaving. Steve, Heidi, and Leah did not struggle emotionally, per se, but they did find themselves growing closer to family and reconsidering relationships once away. Of these five students, Steve, Isaac, and Hannah all recounted a generally close, tightknit family connection at home, and Hannah particularly denoted a strong generational association (i.e., ancestors in the American Civil War). These three students also had among the strongest connections to a family farm or land (explored further below). Leah and Heidi had less land and generational attachment. Leah had a grandparent who knew the Hatfields and McCoys, but denoted a lack of generational persistence, keeping her from fully identifying as Appalachian. Heidi did not report an extensive generational association. Though when asked what parts of the Appalachian culture she identified with, she included, "the family oriented part" symbolizing a connection to the culture's valuations of family.

Conversely, Dianne, Emma, Jared, Mark, and Megan did not recount particular challenges with leaving home. Jared reported no identification as Appalachian. Mark, though, did go several hours away for college and reported some Appalachian identity. Though, he ultimately explained that he "didn't... really grow up in... a rural area" and even though he does have an Appalachian identity, saw it as less than 100\%. Meanwhile, for Dianne, Emma, and Megan, each had somewhat strong identification as being either West Virginian or Appalachian, and Dianne and Emma recounted a strong generational identity. Moreover, each of the three stayed relatively close to home for college (Dianne \& Megan $<$ one hour and Megan $<$ one and a half hours away). Dianne and Megan specifically made note that their college was not, or did not seem, that far from home. In short, for these three students, distance to college may have been a factor in how they responded to leaving and being away from their families. 
Connection to Community and Place: Throughout several of the interviews, students made reference to or noted a farm or a plot of land that was held in high regard or was somehow associated with their family and upbringing. Some students also talked about the potential to someday return home or to be nearby that location. Dianne, Mark, Megan, Steve, Hannah, and Isaac all made a reference to a family farm or plot of land. To a less extent, Dianne and Mark, perhaps intimated the weakest or most subtle connection. Mark grew up in a south-central area of West Virginia, but had a second family home in a remote area of Randolph County, which is in north-central West Virginia. The house had originally been his father's and the family spent time making trips there. Although, he did not grow up rurally, he stated, "I think that... helped me develop... that identification and that sense of connection to the state at an early age." Dianne discussed a large blended family living in one farmhouse. She described no internet and limited television channels making it a necessity to spend time outdoors with her family.

Megan, Steve, Hannah, and Isaac drew a more direct connection to their family's land. Of note, in the previous sub-section it was recounted that Steve, Hannah, and Isaac recognized changing family relationships. Megan did not recount changes, per se, but also denoted it may have been different had her college been further away. She was asked how much or in what ways she identified as Appalachian, to which she replied that she did identify as Appalachian and added, "I grew up on a farm in a rural area." When pressed on if she saw being rural and farming as a component of being Appalachian, she confirmed that she did. Later, when asked if she would consider practicing medicine in rural Appalachia, she explained some reasons why that may not happen, but she also described the pull of being back close to her family and her fiancé being from her hometown. She concluded, “my dad has already offered us a farm, so I don’t know." 
Hannah, who plans to practice in rural Appalachia, saw it unlikely she would actually ever return to her hometown and family farm. In large part, she is drawn to the Eastern Panhandle because of her partners' work in the Washington, DC area. Nonetheless, her family farm was an important consideration for her. When asked about her identification as Appalachian, she noted, "all of my family lives within a few miles of me growing up, my... grandparents all live there." She went on to describe her grandfather being raised close by to where her family currently lives. Her grandparents bought land and much of her extended family lives on or around the property. She established a tight bond with her family and particularly her grandparents, where she spent most nights during elementary school. As indicated above, she struggled to leave for college, and particularly lamented leaving her grandparents. At one point she ultimately added, "I feel like we're very connected to the land... and the people around us." Isaac and Steve, both first-generation students, explained how their families were tied to a piece of land and the connection they also personally felt to that area. Isaac described growing up on "a hay farm." When he was asked about his connection to the farm he talked about his parents. "I mean they're never going to leave it, that's always going to be home no matter where I move." After a probing question regarding the location of his extended family, he explained that the majority was around that area or at least in the county. Steve, who grew up on a dairy farm, described a similar family dynamic, which was partially recounted in the discussion of generational association above. "Yeah, so every one of my neighbors is pretty much related to me somehow." He intimated family holidays and gatherings on the farm as well as get-togethers to accomplish chores. Steve also described his identity as being tied to farming as well as coming back to work the dairy farm that his grandparents had started. 
In sum, Dianne and Mark have weaker connections with place and family. Dianne described a family farmhouse growing up, but she also recounted aging parents as well as siblings who have since moved away. Mark though, who lacks a fully Appalachian identity because he did not grow up in a rural location, has leveraged his second family home in rural West Virginia as a factor in developing a connection to the state and region. Megan, who grew up on a rural family farm, sees her upbringing as a part of her Appalachian identity, which continues to be a factor in her potential plans for the future (further explored in a subsequent section). Hannah has a strong West Virginian identity, which includes generational associations and connections to family land that is home to several generations. Steve and Isaac, although rejecting some aspects of being Appalachian (e.g., riding around on a side-by-side or wearing camo), have a somewhat strong identity, and feel particularly closely connected to their family farms. For these students, where they grew up, the land, and those connections to family have been and continue to be persistent considerations.

Conclusions: As informed in the previous sections, family proximity, extended family associations, and generational associations are important considerations for these Appalachian students. These themes became evident in student discussions of transition to college. Some students specifically discussed how they struggled to part with and be away from their family. Some of these same students, plus others, described how their familial relationships were strengthened by being away, and the distance reaffirmed their family values. Of note, these changes were less prevalent and obvious when students discussed their motivations and transition to medical school from undergraduate programs. Participants had already left home, completed college, and been away for an extended period before medical school. As discussed in the following sections, some students did experience questioning family members or derision 
from extended family when starting medical school, but those moments and experiences seemed less impactful. Moreover, no student necessarily indicated that going to medical school altered a family relationship.

Several students recounted an association with land or a particular area. These two concepts - family and land - tended to comingle: generational associations were evident; family ties were strong; and, connections to a particular place remained important. These connections were of varying degrees, and some students, such as Jared, Leah and Emma, did not necessarily indicate any connection to one single significant location. However, for others, such as Megan, Steve, Hannah, and Heidi, specifically, there was a prevalent undertone implicating family networks and a physical place that were shaping or at least served as a consideration in their future decision making related to practice plans and location. For example, Steve affirmed a desire to return home to work the farm; Isaac asserted his family's hay farm would always be home, even if he ultimately chose to live elsewhere; and, Hannah described the importance of ancestral property to her extended family's living arrangements.

I feel like the people, like family means a lot here and not just your like your... parents and your grandparents, but like your extended family as well... Because all of my family lives within a few miles of me growing up... My grandparents all live there... My grandfather was a boy on a farm that was just like 10 miles from our house, so I feel like we're very connected to the land into and the people around us.

Although students experienced challenges in the first and second years of college, such as emotional struggles with being away from home, those seemingly abated in intensity by the time they transitioned to medical school. However, some of the pulls back to particular places, which are associated with generational identity, community, and extended family remain prevalent or at 
least a constant consideration for these students into the fourth year of their medical programs. Although students may better cope with leaving and being away from home, some of these aforementioned considerations remain as students near graduation from medical school and future practice. This theme largely informs latter findings, particularly in Chapter V, regarding why some Appalachian medical students may decide to return to or altogether leave their home communities, or struggle with the dissonance between the two options. Although students may better cope with leaving and being away from their home, some still struggle with the internal conflict of being away. For some students, feelings of cultural attachment, associations, and a desire to be close to familiar family and communities resurface later during medical school, which is introduced in the next theme.

\section{Theme 2: Cultural Associations Resurfaced During Rural/Community Rotations}

Medical students are required to complete eight-weeks of community or rural rotations for graduation. Four of those weeks are completed during the third-year of the program as part of an eight-week-long family medicine clerkship. Rural rotations can occur nearly anywhere in West Virginia that a faculty preceptor is practicing. However, students may not rotate in a few of the more populous areas of the state including Charleston, Martinsburg, or Morgantown. Many students often find preceptors near their hometown so they can spend time with family, are familiar with the area, or because of the practical nature of staying with relatives. A similar experience, a community rotation, is required in the fourth year, but it is not within a specific clerkship or rotation. Instead, the rotation is its own standalone experience, and students have more freedom to work in various communities and in other specialties besides family medicine. Although some experiences are hospital based, many are located in outpatient primary care. Though students may not rotate at any of the three previously listed locations, they may rotate in 
other populous locations in the state.

During the interviews, I asked

students about both rotation experiences.

Many participants intertwined both

experiences because they commonly

rotated at the same clinic in both the third

year and the fourth year. Students in this

study rotated essentially all across the

state and received a multitude of clinical

experiences. Some examples of where

students in this study rotated include, but

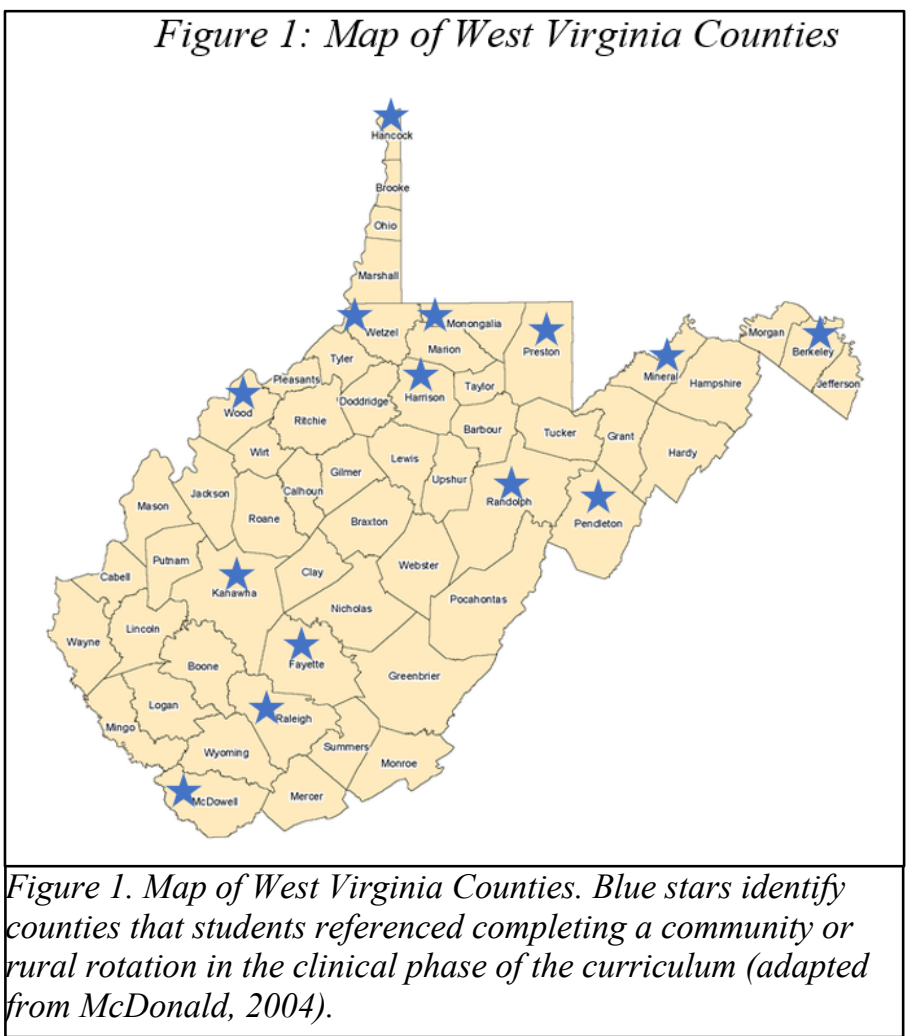

are not limited to, Berkeley, Fayette, Hancock, Harrison, Kanawha, McDowell, Monongalia,

Pendleton, Preston, Mineral, Raleigh, Randolph, Wetzel, and Wood counties (Figure 1; Table 3).

Nearly all students spoke highly of these experiences and their benefit. In particular, students

discussed that it was important to

see patients in their home

environment, which would allow

them to better understand the

patient population and how

physicians engage that

Table 3

County

Berkeley

Descriptor

Fayette Mineral extractive/coal

Hancock Northern industry; Ohio River commerce

Harrison Agrarian; Populous (Clarksburg/Bridgeport area)

Kanawha Chemical industry (historically) \& mineral

extractive/coal; Populous (Charleston area)

McDowell Mineral extractive/coal

Mineral Agrarian \& timber industry

Monongalia Populous (Morgantown area), major university;

Mineral extractive/coal

Pendleton Agrarian, timber industry

Preston Agrarian, mineral extractive/coal, timber industry

population. It was not uncommon

Raleigh

Randolph

Mineral extractive/coal; Populous (Beckley area)

for students to assert that the

Wetzel Natural gas extraction, Ohio River commerce

Wood Natural gas extraction, Ohio River commerce;

experience was especially Populous (Parkersburg area) 
beneficial for out-of-state or non-resident students. A comment Mark made exemplifies many of these points:

Especially for people who come from out of state, ... I think sometimes it's easy to forget when you're... at [college] and you have such a big hospital, there it almost feels like a big city hospital... and you don't really understand where all these patients are coming from.... it's huge to have that... ground level interaction with people.

Emma also noted, "It's good to see what people experience, who... aren't just coming to... a big academic hospital... it's good to see... what access to care that they have."

In most instances, students were asked about or discussed the patient population they interacted with, and were usually asked a probing follow-up question to gauge how much students identified with that population. Of note when asked if or how much they identified with the patient population, Mark, Jared, and Leah all noted either limited or qualified identification. Jared, who notably has no plans to practice in rural Appalachia, stated, "I never really quite fit in with...West Virginia or [my home] county so much, but that doesn't mean that we couldn't find common ground and that I didn't have a good experience.” Mark and Leah gave more specific examples. Mark talked very highly of his rural experience and said it helped him confirm a desire to practice in rural Appalachia. He also explained that he struggled to identify with the population.

I really don't in a lot of ways. I think I have a good understanding of the culture and the beliefs, but at the same time,... I think some of the reasons I like the state and why they like the state are different,... I think we both like it because it's our home,... and part of it is just me being younger than a lot of the people I met, but I think overall,... politically, 
probably and,... we're probably opposite on a lot of things. But I think that's kind of where we find common ground is... we love the state.

Leah also recounted limitations in her identification with the patient population. "The subset of patients I didn't really relate well with were the... people [that]... would probably consider themselves... identity wise, Appalachian... true and true, like they've had generations here." She gave specific examples of patients who failed to seek medical care after farm accidents or had untreated cancer. She continued, “I don't think I could personally identify with that resistance, or just... I don’t wanna [sic] say lackadaisical but, just like independent streak...”

Although Leah, Mark, and Jared all said they did not, in some way, identify with the patient population, they still described understanding the population. Part of this was attributed to having a personal connection with the community, already. As noted, students commonly return home or to familiar communities for convenience. For example, Leah explained, "I knew a lot of people... so it felt very much like my community." Mark described seeing and treating people he was already acquainted with in the community and the cordial relationships he had developed. Even Jared, who noted that he did not feel like he fit in, was able to say that he knew the patient population, who he described as "pleasant,... open, trusting people, kind of what you would expect from my hometown."

Despite these more limited connections, a number of students, particularly Isaac, Megan, Steve, Dianne, and Heidi, felt a strong identification with the patient population. Isaac, for example, noted, "I was able to relate more to the patient population being out there." He felt this familiarity influenced his preceptor's perceptions of him as a medical student:

I'm sure he was happy to have me as... opposed to a student that was not as familiar with the area. Someone who understands the jokes, someone who understands the... 
intelligence level, someone who understands the difficulties [these patients] maybe have gone through, because... I've seen them, I've been around it my whole life, someone who knows their way around a hay bale type of stuff.

Heidi made a similar comment, stating that she felt comfortable on her rural rotation and being with patients. "Given my culture... growing up in southern West Virginia,... it was just kind of nice, I'm just like talking with people back from home..." She went on to explain that she felt as though she was able to communicate easily with patients and understand colloquialisms and idioms.

Steve and Dianne both specifically mentioned the personal connection that they felt when engaging this population. For example, Steve noted, "You see your parents in them... because... my parents come from the same population, so you know, there's like a farmer who was coming in with complaint $x$ and $y$ like, 'Oh, there's just my dad waiting... nine months to go see a doctor..." Dianne explained, "I was like, these people were just like my parents, my aunts, my uncles, and I felt that kind of deep connection, we're all from West Virginia, kind of feeling." Moreover, Steve talked about seeing people from the community the he knew and for those he did not know, he was able to make personal connections. He, similarly to Isaac, gave examples of understanding limitations of living in a rural community. For instance, he described prescription access limitations and how he may better understand the thought process of patients trying to access limited resources. Dianne made similar comments and discussed automatic connections that she had with her patients, noting, "It was a lot of déjà vu, because I was like, okay, this is... like my hometown... these are the same type of people." She explained that it is vital in rural medicine for the physician to be able to understand how to connect with the patient. She said doctors have somewhat of an authoritative relationship with their patients, and it can be 
level setting for the patient when they find out the physician is from West Virginia or a rural area. "When they find out that you're from the same roots... they're trusting."

It was evident that many students identified with the patient population when they completed their rural and community rotations. They also commonly recounted the experiences as being important for outsiders or students not from West Virginia or Appalachia. Some students, particularly Mark, Jared, and Leah found that they could connect with understand their patients, but they did not always identify with them. Of note from the first section of this chapter, Jared noted that he did not identify as Appalachian. Mark and Leah did identify as Appalachian but also offered some caveats including Mark explaining he did not grow up in rural Appalachia and Leah explaining that her family had not been in the region for generations. Others, including Isaac, Megan, Steve, Dianne, and Heidi, did sense an identification with the patient population. They described understanding the population and even drew parallels to traits of their family. One tension, though, is that even some from this group, as explained above, offered caveats when describing their Appalachian identity. Nonetheless, they intimated a strong connection with this patient population. It was evident that regardless of what caveats this student sample gave when describing their own identity, a number still identified with the patient population, particularly seeing them in their home setting. Moreover, for the students who indicated that they did not identify with the patients as well, they often qualified that they still understood them, the culture, and were able to connect.

When considering the research question regarding how students interpret home and cultural associations as they transition through medical school, the rural and community experiences present a time when students are able to engage with a population and with communities that they may already be a part of or at least can connect with. Moreover, many 
students saw these experiences as pivotal moments in their path. Mark, a soon to be obstetrician and gynecologist, joked that he had a "kneejerk reaction" about his experience. "After that month I was... going to do family medicine and I was... going to go back and work at that clinic... I still feel that way, to some extent, maybe not as strongly but it definitely did not dissuade me." Isaac noted, "It kind of grounded me again... it kind of just made me not forget really where I come... from and like, 'hey these people still need help; don't forget about 'em." Megan gave an example of her rural preceptor showing patience and reexplaining something to a patient that they did not understand. She noted the providers were very connected and understood the population. "How they've done within their practice is really awesome to see, and it's something that I would like... from my practice later."

In sum, these experiences allow students to engage a population they know through a new or different lens. As a region with healthcare shortages and inequities, students rotating through these communities are able to reengage cultural associations with which they are, at the very least, familiar. Although some students explained that these experiences were important for non-Appalachians to better understand a diverse patient population or to better see Appalachian healthcare shortages and disparities, they also described how it benefitted them personally by being able to connect or reconnect with cultural associations. These students were well positioned to make those connections because they understood the population, community and culture, even if, in some ways, they did not fully identify with every aspect of Appalachia. As argued in the previous subsection, some of the students in the sample experienced emotional turmoil upon leaving their home and close family networks, to which some, in turn, saw that departure as strengthening bonds with parents and family despite the departure. In medical school, there seemed to be less turmoil with family and home - those ties had already been 
broken. However, when students returned to their home communities or to other rural locations, such as Isaac, Steve, Megan, Dianne, Leah and Heidi, saw themselves clicking with the environment. Even if they rotated in a rural area that was not their hometown, students seemed to feel belonging and find value in being there. The following comment by Dianne about her rural experience exemplifies this argument. "I'm not saying that you have to have grown up in a rural area to succeed in rural medicine, but... having the background of having grown up in rural West Virginia was a benefit to doing that kind of medicine."

\section{Research Question II: Within this student population, who or what do they recognize as facilitators or impediments to their academic journey?}

\section{Theme 1: Facilitator: Parents and Family Members}

The postsecondary academic journey begins well before medical school. All of the medical students in this study asserted they either always knew they were going to go to college or at least realized they would pursue college from a very early age, regardless of first-generation status. For some, this decision was intimately connected to early desires to pursue medical school.

Of the four first-generation students, Isaac and Dianne concluded they were destined for college very young. When asked when he had decided he was going to go to college, Isaac noted:

Five, I mean I was always going to college,... I knew from a young age that I was going to do that... It wasn't something like that, you know, if I could... it was more just where and am I going to be able to afford it type thing.

Dianne similarly noted, "Unlike all my other brothers, I was destined for college ever since I was a little, tiny kid. ...Everybody knew that... I would end up in college and graduating." However, 
Steve and Megan were perhaps the oldest to decide they were going to go to college and linked their decision to determining they wanted to study medicine. Megan volunteered at the local hospital in middle school where her mother worked as a nurse and decided she would like a career as a physician. Steve described deciding to go to medical school after being diagnosed with juvenile diabetes and explained that his pediatrician and subsequent physician interactions were major motivations for medical school.

However, all four of the students described their parents as being a major motivator for pursuing college and several remarked about the high value placed on education in their home. Megan noted, "in elementary school and middle school... they were always very involved in helping me study and,... I think, since they didn't go to college, you know?” Megan did note some snide remarks from extended family when she decided to go to college (recounted below), but she did not indicate anything besides full support from immediate family. Steve, whose mother completed a two year degree, similarly noted:

My mom was a dairy farmer and they really didn't have much money, but the values that her parents had instilled in my mom and that she wanted to make sure we were given opportunity, so they made sure that they could send us to a private school... we don't have new vehicles but we went to a private school.

He followed up by stating that his parents often paid him to read books and as a reward for good grades on a graduated scale. He said in college he decided on pre-medicine adding about his parents as motivators, stating, “They're so proud of you, you never want to let them down, so you just keep... that up." Isaac, whose father completed some college also talked about not wanting to upset his parents. When asked about his biggest motivators for college, Isaac stated, “My parents and my sister probably.... didn't want to upset the parents... or not upset them but 
disappoint them, and just wanted to make them proud." Finally, Dianne, recounted her father always taking her to checkout science books at the library. When she struggled in college math and science, he tried to persuade her to stick it out and not change her major.

Of the six non-first-generation students, all similarly recounted knowing they were destined for college at a very early age. As a few examples, Jared noted, "Ever since I was like a child my parents are... talking about college was [sic] just an extension after high school.” Mark stated, "I think it was just kind of always understood that I would go to college." Emma explained, "I kind of always thought I was gonna [sic] go to college..." She further explicated that her parents being college graduates made the trajectory seemingly more obvious. Heidi's comments were similar, "I think it was kind of always just the thing like, you're going to college." She also relayed that it was just an expectation from her parents. Although not every student recounted specifically how their parents influenced the actual decision to go to college, the majority recounted the positive influence their parents had on their educational path to some degree. Moreover, five of the students used the term "always" when describing when they decided to go to college. Other examples of similar language include, "ever since I was a child," "I was destined for college ever since I was a little tiny, kid," and "I don't know that was ever a question."

For some students, one parent served as a more powerful motivator than the other, though this was not uniform across participants. Mark and Hannah's fathers both completed high school while Mark's mother had a four-year degree and Hannah's mother completed a doctorate. Both remarked that their mother was more stringent with their academics and educational progress. Mark noted his mother keeping track of his academics and stated, "My dad... he didn't care what 
we did, as long as... we were happy and... acting right." Similarly, Hannah noted the influence of her mom on her postsecondary aspirations.

They were different... my dad never pushed me to get good grades or anything like that but he always told me like... 'anything you want to do, you can do it'... but my mom was the do-er... my dad worked really hard, but my mom was the one who was like, education.

She described her mother as being the first person in her family to graduate from college. Hannah concluded that her mom likely would have gone to medical school but, "being a doctor was like something women just didn't do in the 70s, at least not in [my hometown]." She saw this limitation for her mother as a motivator for her to pursue medicine. Megan, a first-generation student, stated that both her parents were very supportive, but then recounted having specific members of her mother helping her with school work, such as spelling words.

Of the seven remaining students, Emma, Dianne, Leah, and Heidi saw their father as either more motivating or had higher expectations, but many still saw their mother as having relatively high expectations. Of Isaac, Steve, and Jared, none recounted one parent being particularly more influential than the other although Megan did recount specifics about how one parent may have helped them with one particular aspect of their academic progress.

In sum, many recounted positive educational views and attitudes in the families. In conjunction, there was a general college expectation among this sample, so much so that they could not recount when the idea ever really surfaced. This expectation is coupled with the examples students gave of their parent's support for education and college. There were differences and nuances in how parents showed their support. Dianne, for example, recounted trips to the library with her father while several students explained how their parents incentivized 
or stressed good grades. It was also not uncommon for students to mention other family members who had gone to college before them, including parents or cousins, and thus college for them becoming the next logical step. No students in this study indicated dissuasion for college from immediate family and most typically recounted lots of support from others. Megan did recount some general mocking from extended family. Although many examples of positivity and support are listed in this section, they are also interspersed and are apparent in examples utilized for other themes below. Moreover, these subsequent sections present aspects of the student's upbringings or educational journeys that were often impacted by parent's decision or attitudes about exposing students to certain opportunities.

\section{Theme 2: Facilitator: Positive Interactions with Science and Medicine}

As students expressed knowing from an early age that they wanted to go to college and, more specifically, pursue a career in medicine, they had a varied set of influencers or interactions with science and medicine throughout their upbringing. No student interviewed had a parent or physician family member; however, some did have a family member who worked in healthcare or for a hospital system. Many of those students recounted how influential specific interactions or moments were to their academic trajectory. Hannah, who studied pre-medicine in college, but held an unrelated career for five years after college, discussed her mother working as a nurse practitioner and earning a doctorate in nursing practice. However, Hannah described the interactions and impacts she had at private Catholic school. She explained, "My life probably would be... very different if it not where I had gone, because I just felt like I was around a lot of... other high achieving students.” Megan, a first-generation student, also had a parent who was a nurse. She explained that her interest for medicine began in middle school while volunteering at the local hospital where her mom worked. She denoted a love for being in the 
hospital. Similarly, Emma relayed that her dad working in hospital information technology made her relaxed in that setting. "I knew I was comfortable... even though he wasn't in the healthcare part of it." She later added, "I would go visit him at the hospital and I kind of felt like weirdly... attached to the hospital."

Many students, including Isaac, Megan, Emma, Steve, and Heidi indicated positive early interactions with a pediatrician, which were noteworthy enough for to discuss those experiences, but with the exception of Steve, no students necessarily recounted these individuals as mentors or explicitly influential. Steve did credit his earliest interests for medicine as a result of positive and continual interactions with his pediatrician:

Pretty much when I was diagnosed with juvenile diabetes and my doctor was just awesome and would explain everything, and you know when you're kind of questioning like, “Oh, what am I gonna [sic] do for a living?” when you're in high school or even like a seventh, eighth grader, she was like, "Why don't you... come sit in the clinic with me and shadow me?"

Steve recounted his interactions with other patients who had diabetes and talking with them. “Looking back, I'm like, oh my gosh, she was kind of like helping me kind of get a little taste of it."

Heidi also denoted an interest that surfaced in middle school. While on a trip to visit family, she attended the Body Worlds Exhibit. She recounted her interest growing from that experience throughout high school and college, but explained:

The spark and everything kind of started in middle school with my parents being like, 'hey look at this' and then I was a 'oh, this is awesome,' then they would buy me books, too, that were like medicine based because they knew I liked it. 
Heidi denoted a fascination with Discovery: Health programming and explained that she spent time watching it with her family. Similarly, Dianne discussed positive interactions with the human sciences early:

When I was a little girl... my dad would take me to the library every week and I would check out stacks and stacks of books and they were always human anatomy, physiology... I was always fascinated with biology and human anatomy and physiology so I can't even remember when I decided I wanted to be a doctor, feels like it was, ever since I was a really little girl.

The interactions outside of their formal education where particularly important for Dianne and Heidi who both explained (and is further detailed below) educational deficiencies or limited opportunity growing up. For example, Heidi noted:

I didn't know... what research was until I left [southern West Virginia] ... or, like all those different opportunities within the science field... Down there, there's... like business or there's... a couple... random trades you can get into, but... as far as other opportunities for different jobs... you don't know too much about it, unless you go seek it out yourself.

She concluded by explaining that opportunities may have been different if she had been raised in cities like Washington, DC or Pittsburgh.

Finally, Leah, Isaac, Mark, and Jared either recounted deciding to pursue medicine later or questioned their path during college. All four of the students remarked that they had no individuals in their family who were physicians and had limited interactions with physicians with the exception of some positive pediatrician interactions. Jared noted that his spark came from glamorized TV such as Beverly Hills, 90210, which inspired an interested in plastic surgery. He 
explained that he had second thoughts in college and considered working at his father's business instead, but ultimately decided that he wanted to pursue medicine because it was more mentally stimulating. Mark similarly questioned his decision to become a physician. He decided in high school:

You know I never really had that sort of figure in my life... so I think most of it just came out of like... sort of the standard... path for, you know, you're good at science and want to help people kind of a thing, just the basic stuff.

However, in college, he was dismayed due to the competitive nature of fellow undergraduate students. He explained that he ultimately decided to pursue medicine again when he was home from college and saw dilapidated community projects he had worked on with a church group ten years prior. He decided that becoming a physician would allow him to make more lasting contributions to the community or state.

Leah originally got the idea to pursue medicine in high school science courses, but did not actually commit to that track until college and after determining that veterinary medicine was not a good fit. She recounted that her extended family was surprised, and that she had no one close in the medical field. Her parents, however, had advanced degrees in science fields. When asked if she thought their background and education may have motivated her to follow a science trajectory, she confirmed that she believed it did. Similarly, Isaac also had limited medical influence and made the decision for medicine in college. He explained that his mother worked in medical records during his childhood, but when asked if they ever talked about the work or the doctor's office growing up, he was dismissive, saying they never did. He explained that he knew he wanted to work in healthcare or become a teacher. His older sister pursued physical therapy as a career. When asked what actually led him to medicine, he explained, "The possibility that I 
could... when I got to [college], I was just crushing classes and doing great and not having to try too hard. I was like okay, maybe I have a chance here."

In sum, the majority of students discovered the potential to become a physician early. However, no students in the sample actually had a physician family member and besides some positive interactions with a pediatrician, most had limited early interaction with doctors, save Steve. A few did recount family members who worked in a hospital or healthcare, such as Hannah, Emma, and Megan. For Emma and Megan, particularly, those led to influential interactions with that environment. Others found inspiration from presented science and medicine materials, such as Heidi and Dianne. A common thread is that the majority of students had meaningful positive interactions with medicine or science well before college, such as parents endorsing science books or programming. The four remaining students - Leah, Isaac, Mark, and Jared - tended to arrive at medicine as a career later and recounted fewer meaningful interactions with science and medicine. Jared, who was first inspired by television, did decide early that he wanted to pursue a career in medicine, but he ultimately waivered and enrolled in a master's degree program to be sure. Leah described having parents in science fields and that put her onto a science trajectory, but not necessarily toward medicine. Mark and Isaac more-or-less recounted as arriving at the decision due to process of elimination (e.g., good at science, academic success, etc.).

\section{Theme 3: Tension: Pre-College Educational Opportunities}

It was not uncommon for students to discuss how their life may have been different if they had been born, raised, or attended school somewhere else. These views can be divided into two competing narratives found throughout the interviews. First, many students described how they were in some way fortunate or privileged in either decisions made by their family or by 
more happenstance of opportunity (e.g., offerings at their school). Therefore, these opportunities are described as a facilitator. However, conversely, as further explained, missed opportunities or educational limitations also served as impediments or limitations to their educational journey. Many of these limitations were realized in hindsight, after going to college or medical school. In several of the interviews, students discussed how they were in some way fortunate. This conversation typically surfaced when students were asked what they saw as the biggest challenges facing Appalachia or their home area. Leah did not always feel 100\% Appalachian in part because she moved to the region at age ten. "I didn't necessarily have all of the struggles that I think maybe children who... come through multiple generations of individuals living here might face". She further noted about children in Appalachia:

The region of the country is known for poor socio-economic status and so... they didn't have as many opportunities, perhaps, as I did. They didn't have parents who perhaps pushed them to go to college... I knew that... was always... what I was going to do... I could see [going to college] being a lot harder to do with maybe your parents having never gone to college or they didn't know how to... navigate the process.

Mark, who grew up in a more populated area of West Virginia and has spent time in a populated area of the state for medical school, shared, "You kind of see a little bit of reflection from the problems that are facing some of the more rural areas... and [I'm] kind of lucky to be enclosed from that a little bit." Isaac, though, who did grow up in a rural part of West Virginia noted, "I didn't experience a lot of the poverty that goes around in the Appalachian area... but I mean further south in our county you do see a lot of it." Jared, a future obstetrician and gynecologist, described the prevalence of both opioid misuse disorder and teen pregnancy in his home 
community. From there, he was asked if he thought the two issues were related, to which he replied:

Oh yeah... I think it's... a symptom of the culture of like not having much to do and what kind of environment you're raised in... I was lucky enough to be raised outside of that world but that doesn't mean I didn't see it.

This conversation of being generally fortunate in upbringing was also discussed in terms of educational opportunity. Foremost, Hannah and Steve, a first-generation student, both attended private Catholic schools, to which they attributed their opportunities as being related to that experience. Hannah remarked that her elementary school and her hometown as "really rural" and "very poor." Through school consolidation, her family opted to send her to a Catholic school in another town. She explained, "I feel very fortunate that I got that experience, but it was very different... the city kids are very different than the rural kids." She continued, "My life... would be very different if it's not where I had... I was around a lot of... other high achieving students, I was pushed to the better." She explained that in private school, college was the expectation and that was not necessarily the case if she had gone to a public school in the area. Some parallels can be drawn with Steve, the son of dairy farmers discussed in the previous section. He also commuted to a Catholic private school, which was about a 25-minute drive into another state. He noted, "My parents wanted the best education so that was kind of like the best option we had." Despite denoting that the educational experience was positive and the best in his area, he also expressed that he felt like there were fewer opportunities for college prep-courses compared to more populated areas in the region.

Other students specifically stated they were fortunate to have positive educational opportunities. Emma noted that she, "felt kind of privileged" with her high school and the 
opportunities it offered. She stated that her high school, "had a lot of resources that a lot of other schools didn't." Similarly, Jared, who lamented the rate of opioid misuse disorder and unwanted teen pregnancy, also stated that educationally, he benefited, making a point to refer to the schools as "excellent" and his high school as "very good... especially the science department." $\mathrm{He}$ explained that he went to college with around a full semester of college credit. Along with Emma and Jared, Isaac and Heidi were also complementary of their educators. Along with seeing educators as positive, a number of students talked about the opportunity to take Advanced Placement, International Baccalaureate (IB), or dual credit courses for college. For example, Mark mentioned starting at the 300 level of foreign language in college because of IB credit. Jared claimed he transferred 15 credit hours to his college because of dual credit courses. Leah explained that she started college as a sophomore, credit-wise.

With these aforementioned examples in mind, many students looked favorably on some aspect of their high school education whether it be having good educators, the ability to take college credit courses, or for Hannah and Steve, the ability to attend a private school. However, a notable tension in the findings is that a number of students, even some of the those previously discussed, also found fault or flaws in their educational experience. First, several of the students still found their college preparatory courses limited. Steve, who went to private school, noted that he received a good education for his area, but it did not compare to, for example, students in Pittsburgh who were able to take a higher volume of college credit courses. Moreover, college credit courses are a major advantage to adjusting well to college. He noted, when discussing students with a lot of college preparatory opportunities, "then they know how to kind of study for college courses, so me coming into college... I really wasn't prepared as much... the only reason I did well is because I have a ton of internal motivation." He went on to explain that some 
students fail out, because they cannot keep up, and, "they... then go back home and do some blue collar job, even though they wanted to go to college,... that's definitely a barrier."

Other students also saw limitations in the college preparatory courses offered. Leah, who started college as a sophomore and recounted eight to ten AP courses in her high school, saw her opportunities as somewhat in the middle. On one hand, she denoted that her opportunities were similar to another high school nearby, but likely much more than other places in the Eastern Panhandle or in West Virginia. But, she also noted that she traveled to Virginia to take AP exams and it occurred to her that those schools, "offered way more." Megan, from a rural area in West Virginia's Northern Panhandle, explained that her high school had basic AP courses. She stated, "We had... AP chemistry, AP history, AP English. But there were... more classes, that I had heard of... when I got to college that... weren't offered at my high school." Heidi, who grew up in southern West Virginia, explained that her high school "offered a couple AP classes" but compared it to a high school in Charleston, which she stated offered "all the spectrum of the different opportunities for AP classes." Similar to Steve, she discussed how this could impact someone's preparation for college. Heidi also made note that fewer college course opportunities can impact a student's grade point average (such having fewer weighted courses like AP courses), which may limit a student for college admission compared to students who had those opportunities. She described taking advantage of supplemental activities to augment her college application. When describing how her and her friends viewed their college applications, she concluded, "It was kind of like, oh well, I've done everything I could and volunteered as much as I could, but we like only had access to these many things."

Perhaps no student was more direct than Dianne about the limitations of her pre-college education. Dianne struggled with math and ultimately decided against medicine and pursued 
another career. She claimed at her public high school, the only advanced math teacher was unconcerned if they knew the content, but simply allowed the students to pass. When thinking she may try medicine later in her previous career, she started post-baccalaureate studies at a university in the south.

I had to face the algebra again and that terrified me, but I was blessed and fortunate to have an excellent... algebra professor. And I worked my butt off in that class and I got an A and I was like, it was not me, that's what I realized, it was my high school. She noted her success was a catalyst for returning to the state and seriously reconsidering medical school. Dianne, a non-traditional student, also discussed how her community has resisted county school consolidation and doubted if the educational opportunities had improved. She noted, "So now, this little, tiny high school with very little funding and only a few students, you know these kids, don't have the same... opportunities as kids going to bigger high schools."

In sum, feelings and reflections regarding pre-college educational opportunities were mixed. Many students saw themselves as in some way fortunate that they received the benefits and experiences afforded to them. Some also praised their teachers or specific aspects of their education. Meanwhile, some of these same students, as well as others, saw their opportunities as in some way limiting as they prepared for higher education. Many students in the study both looked to other communities or areas to help elucidate ways their education provided fortunate opportunities, but in also what ways it was limiting (e.g., comparing the number of college credit courses at their high school to other areas). This finding creates a dissonance, both between and within students, regarding how they perceived and reflected upon their educational opportunities. A caveat, though, is that all students in this study are academically or educationally high achievers. They have all been successful in leaving their hometown for higher education, being 
accepted to medical school, and are nearly physicians. That is to say, with the possible exception of Dianne who saw her aspirations derailed for period of time, none of the students in this study failed to become a physician because of these limitations, despite any perception they may have. Leah summarized it well by noting:

I'm going to be an MD in a month or two, so I really can't think that I missed out on too much or like I didn't have opportunities. I think they were different opportunities, but I think... there are still like pathways to where you want to go.

Nonetheless, the argument could be made that indeed, all the aforementioned students were successful in terms of completing medical school, but that does not consider students outside the scope of this study who either failed to aspire or aspired and failed to achieve this high level of education, perhaps in part because of educational limitations.

\section{Theme 4: Impediment: Questioners}

All students were asked about their decision to attend medical school and how it was received. Overall, most students recounted support from family, but some did explain that their decision was questioned. The questioning ranged from wanting to be sure they understood the commitment of time and money that would be required to achieve a medical doctorate up to more general derision from extended family.

Some family members questioned if the career path would be worth it, or if there was a similar path that could require less time and cost (e.g., becoming a physician assistant). Isaac explained that his dad wanted to be sure he was making the right choice. He said his dad asked, ““Are you sure you want to do this?', but it never was a negative way. Like it was always just like... 'I hear it's a long time, it's four years, it's a lot of money."' Similarly, Heidi denoted that her father really wanted her to "just... be sure" and presented other career paths like nursing, 
physician assistant, and occupational therapy as options, but he did not discourage her path. She also explained that more extended family members, including grandparents and cousins, made questioning comments about the length of medical school and the opportunity for other career routes. She noted, “They weren’t like direct comments, but just kind of... little things that were like, 'Oh, are you sure?'”

Dianne, who worked for an extended period in another career explained that the questioning she received was direct and straightforward. She was working in a lucrative field in another state, and she noted her best friend at the time was not pleased with her decision.

Most people would have thought I had the perfect life, so when I said I'm going to throw it all away and pursue this crazy dream... I could tell she was not that thrilled, she thought I was making a terrible mistake.

She went on to explain that her parents also questioned her decision. Her father, who had originally encouraged her not to change her major in college and continue pursuing medicine, asked if maybe she had considered becoming a physician assistant.

Megan and Hannah also experienced some questioning from family members. Hannah recalled many supportive family members, but she explained that her grandmother did question her decision. She explained that her grandmother has gender-based expectations and noted:

...[my grandmother] still thinks that women should be at home with, and taking care of their husbands and their children, and my grandmother told me all the time just like, 'don't you wish you wouldn't have done this?' like, 'this was a big mistake' and so she was like never really encouraging about it, she was more like, 'it's okay, you can quit.' Megan did not recount direct comments from immediate family but did intimate some derision from aunts and uncles. 
They were kind of like, 'Oh, you're going to college?' kind of thing and I definitely felt that when I was deciding on medical school... like, 'Oh, you're going to be a doctor?' like, 'first doctor in the family ever' kind of stuff... but never anything that was... harsh. In sum, when making the decision to go to medical school, a few students denoted various levels of family questioning that ranged from generally wanting to express the possibility of other opportunities up to more derisive comments. Although most generally recounted support even in light of some questioning. It is worth mentioning that three of the five students who experienced some type of questioning were first-generation students, including Dianne, Isaac, and Megan. Moreover, four of the five students were women, including Dianne, Hannah, Heidi, and Megan. For several of the students, the potential to pursue other careers in medicine were a part of the conversation. These findings are explored further in Chapter VI; however, questioning related to length of study and cost seemed to convey concern for risk. Such as, parents were considering the ideas of an extensive medical education with a heavy financial burden when there are other suitable and reputable career options. Moreover, as Hannah most explicitly described, some questioning had gender-based undertones.

\section{Theme 5: Facilitator: Themselves \& Self-Motivation}

It is important to denote that some of the students, particularly Jared, Hannah, Isaac, Steve, and Heidi, explained how hard they worked or that they felt a strong sense of personal motivation to achieve or succeed. When asked who he considered to be the biggest motivator to go to college, Jared noted, "Myself, my parents, I mean I would say my parents motivated me, [it] was just an expectation... I was my biggest motivator." Hannah made a similar, but less straightforward comment. She reflected, "I was always much harder on myself than my parents were on me.” 
Isaac, Heidi, and Steve all specifically discussed having a strong desire to do well and to set themselves apart. For example, Steve, a first-generation student raised on a dairy farm, explained that the enjoyed being at college and he felt driven to achieve. He talked about graduating summa cum laude and noted:

I definitely didn't utilize my free time as much as my friends did. I was often in the library a lot later than them studying. When they'd go out to parties or something, I'd just be studying so I definitely... had to earn my way to get to medical school.

He went on, "the only reason I did well is because I have a ton of internal motivation just to do as best as I can."

Heidi, from southern West Virginia, described her strong work ethic when she went to college, which was complicated by weaker study skills that she was able to resolve. She noted that her and her friends who were wanting to be in medicine were, "all like trying to push and always working hard." She explained that college "is what you make of it" and that it is possible to get by with the bare minimum, but she went on to explain needing to, "put in the extra effort."

Finally, Isaac summarized how he saw his educational experience from high school and in college. He described what he perceived to be good educational opportunity at his high school. Moreover, he explained, "but you had to be self-motivated and a lot of people that... came from a different family background, weren't self-motivated and they did not take advantage of... furthering their education." He went on to say that he and a group of his peers: want[ed] to do something more with our lives, so it prepared me because I made it prepare me... The opportunity is there to get out, but you just have to know that's what you want to do and work for it and not just let it pass you by. 
To summarize, it is worth noting that although students gained and recognized a great deal of family support for college and motivation to be academically high achieving, many also recounted putting a great deal of pressure on themselves. It is possible and likely that a lot of this pressure perhaps first surfaced from positive early feedback from parents. Although students could recount positive family influences, interactions with an educational system, or non-formal educational experiences which motivated them to do well in high school and college, students also adopted high expectations, which they recognized as leveraging for success.

\section{Theme 6: Facilitator and Impediment: Community and Rural Physicians}

As noted in the previous theme, many students had limited interactions with actual physicians during their upbringing. As students advanced toward college and medical school, many began to engage physicians in the community for shadowing and mentoring. When students were asked if there was anyone they could think of who dissuaded them from medicine, four responded with a specific example of an interaction they had with a doctor in the community. Those interactions were of varying degrees of dissuasion from seemingly joking up to more openly discouraging medicine as a career. Conversely, though, students also relayed particularly important interactions as motivating or beneficial. Seemingly more in jest, Isaac noted that his pediatrician told him, "to go be a pilot" noting his good vision and that, "medical school's rough". However, he also had a particularly important experience with another doctor: "I shadowed a surgeon... that did an operation on my dad that ended up saving his life." After then mentioning that he did not have a lot of influential physicians in his life, he said:

You know that was a cool moment... that actually made me consider surgery for a while but it was just more reason, like... you can really help people, no matter what field of 
medicine you're in, and so I guess... it motivated me a little more and then I just wanted to be in medicine.

Jared and Emma recounted more discouraging remarks from physicians in the community. Jared mentioned that he had discouraging interactions with clients at his father's business who were physicians. He noted:

It wasn't that they were saying not to do it, but it's exactly what I tell people that are considering medicine... I wouldn't even say labor of love, it's just a hellish path to a career and like you have to be absolutely dead certain that... you don't want to do anything else.

He further stated that he did not know if they were really trying to dissuade him or to caution him. Emma noted, "I actually do remember shadowing a couple physicians who would kind of, say,... 'oh you want to go to medical school?' and they'd be like, 'don't... go to medical school' like just because it's really hard." As a probing question, she was asked if she thought they were discouraging or if perhaps they were counseling her on the challenges in the path to becoming a physician. She replied that she could see the interactions as, "a combination of the two." She recounted one physician being a neurosurgeon, an intensive and demanding specialty, while the other was an allergist who did explain that medical school was challenging, but coupled that notion with the benefits and satisfaction of completing the degree and working as a doctor.

Hannah, who had a previous career, however, recounted that some of the remarks she was subjected to actually led her away from medicine for a period of time. She said, "I wanted to go to medical school after college, I just got talked out of it by doctors... several doctors I worked with said don't be a doctor." Much like Emma's description of the neurosurgeon she shadowed, Hannah suspected that perhaps the physician's specialty may have been a factor. She 
noted that they were retinal specialists, worked long hours, perhaps did not make as much money as they once did, and were unhappy. After college, though, she did shadow physicians who encouraged her to go to medical school. She noted, "I was just talking to the wrong people, people who just weren't happy with their... practice of medicine.”

Despite the fact that some students indicated either mixed messaging or dissuasion from physicians, not all interactions were in some way negative. As exemplified above, Isaac saw a surgeon who operated on his father as very influential. Steve, the student diagnosed with juvenile diabetes, also had particularly positive interactions with a physician. In fact, in the following years, he was able to recount other positive physicians, some of which became and continue to be a mentor. The argument to be made is that as shown in previous themes, many students had limited direct opportunities to engage the medical field. These interactions are particularly important. As the students showed interest in the field and attempted to engage medicine, it was complicated by mixed messaging. However, a notable tension is that many of the doctors were not overtly discouraging, but wanted to make a point about the challenges in medicine, which may have been particularly related to their specialty. Moreover, as already argued, this group of students already lacked regular interaction with physicians and were generally interested in knowing more about the expectations of what it would take to be a physician. To exemplify this point, Leah noted:

I don't think I was told exactly how hard medical school is going to be... I almost wish like I did have someone in my family who was or... like I knew who was a physician, just so they could be like, 'yeah,... I know school hasn't been hard for you before, but like this is hard.' 
It did not seem as though this sample of students necessarily objected to hearing about the pitfalls and challenges, because there is evidence they welcomed it. However, some of the interactions had mixed messaging or edged on deterrence instead of establishing expectations. 


\section{Chapter V: Career and Place Aspiration}

\section{Research Question III: What motivates some students to stay or leave Appalachia or WV?}

\section{Theme 1: Motivation to Stay: Characteristics of Rural Lifestyle}

During the interviews, all students were asked what they saw as the greatest challenges in the Appalachian region, which was followed up by asking what they also recognized as the challenges facing their home communities. Commonly, among other issues, students made references to some aspect of isolation or connectivity, including broadband issues. This was particularly important when students described the limitations of tapping into telemedicine as a statewide resource. Others made note of their particular experiences with limited means of communication or isolation. Heidi specifically described her fourth year community experience in a town with no cellphone or internet service and patients borrowing the clinic telephone. She described the patient population relying on mediums like the newspaper for information.

Despite the notable limitations in both West Virginia and Appalachia, there were aspects of rural life that were particularly appealing or important to students. Hannah described being a rural person, but her spouse being more of a city person.

We didn't really have, other than my family, like neighbors... and so I like living... where I don't have to worry about [a homeowners association (HOA)] like I live in now, and if you want a chicken in your backyard you can have some chickens in your backyard, and a clothesline, and the HOA isn't going to tell you take it down. Isaac also made note of desiring some aspects of rural life he favored. He described maybe someday returning to his home community stating, "I mean it's... far away from everybody, you know, I never grew up with neighbors and I don't want to.” Also, Steve has notably been referenced numerous times wanting to return to the family farm. Even though he is leaving the 
state for residency, he plainly stated, "I'm going to return home to the farm, just because that's where I want to be."

Being able to connect with like-minded or rural-minded physicians were important factors for some students who desired to practice rurally or had a rural identification. Several of the students, particularly Isaac, Hannah, Steve, and Mark described having role model physicians who showed them that they could have a successful and decorated medical career while living or practicing in a rural setting, which helped reaffirm that possibility. Mark described a rural physician who had left, received advanced specialty training and came back to practice rurally. He noted that after he heard that particular doctor's story, he surmised, "anyone can come back to West Virginia." Isaac noted a particularly influential doctor also who he described as having a farm while also practicing medicine in the specialty of his choice, having a decorated career, and working at a larger medical center. Isaac noted that the physician challenged him to ask himself what he really wanted to do with his life. Moreover, he explained that his mentor worked hard to lead to the lifestyle he wanted, saying, "It's just a great setup and I think that would be great to come back to." He followed up by saying he just was not totally sure if he wanted to live in the state his whole life, but may perhaps try out living somewhere else—particularly rurally—but still come back someday. Steve also described an interaction with this same physician, noting that he exemplified being able to maintain a rural lifestyle but also be an accomplished physician. He stated that the physician, "has a farming background, and... he works his butt off, but still is able to maintain a farm." Steve also indicated just how influential farming was to his decision making. For example he explained, "Every time I talk to an attending who is a farmer,... owns land and some animals, I definitely feel a type of connection with them. Even on [residency] interviews... I can just connect with them." 
Although students were able to identify many of the challenges or limitations present in both the region, some also recognized things like isolation or independence as reasons for potentially practicing rurally. These were aspects of a rural lifestyle they were accustomed to and valued. Of note, Isaac, Steve, Hannah, Dianne, and Mark recounted specific interactions with providers that stoked their rural interests, or at least allowed them to recognize that rural life and practice may not be as limiting as they expected. This theme is particularly related to rural lifestyle, but is closely connected to the subsequent theme: Career Implications and Perceptions, as it describes how some students desired or were drawn to aspects of rural medicine.

\section{Theme 2: Motivation to Stay: Desire to Serve or Give Back}

For some students, the desire to serve or give back to the state and region are major factors in determining where they may ultimately practice medicine. Hannah, succinctly summarized by stating, "I feel like we feel a sense of responsibility to making [West Virginia] a batter place." Mark was considering alternative career paths in college, partially because of personality conflicts with other pre-medicine students. However, the idea of helping and serving the people of the state reaffirmed his desire for medicine. He explained that one summer when he was home from college he visited a community that his church camp had provided mission services. He further articulated that he found those projects becoming decrepit, which prompted him to find a way to create something more lasting. He noted that by becoming a physician and serving in a community, he can build relationships and create something more enduring. He later noted:

I just feel obligated to serve the state... I guess growing up here you constantly are... hearing about how we're last in everything until recently with... the [COVID-19] vaccine... and you know there's no reason, like people live in West Virginia or other like 
similar areas in Appalachia don't deserve the best type of healthcare, or whatever it is, like everyone else in other part of the country and... that's been my belief... It was like slowly cultivated throughout the years and now it's just sort of this like unshakable thing in the back of my mind, like I would feel like a betrayal, even if I was like next door in like southwest Virginia, or like eastern Kentucky.

Several students echoed this sentiment. Steve, with a strong desire to return to his family farm talked extensively about his identity as an Appalachian being tied to helping his neighbors and community. He used the example of helping save a neighbor's hay from a coming storm and riding his four-wheeler out to lead a neighbor's cow back to pasture. He later explained living rurally is a preference for some people and he rhetorically stated that some individuals may wonder why high achieving physicians may decide to practice rurally, to which he a retorted, "it's like well, rural people need good care too." Others made note of reconnecting with a rural population during their third-year rural family medicine rotation. Isaac, who went back to his home county noted, "it kind of just made me not forget really where I come... from and like, 'hey, these people still need help, don't forget about 'em."” Dianne talked about connecting with the people during her rotation in the Eastern Panhandle, stating that she had already decided wanted to stay in the state and practice, but she noted, "that was like, okay, yes, this is something that I'd like to do that, you can really make a difference."

Although there are other examples of students noting their desire to help and serve the population, those relayed above are exemplary of the sentiment that many students relayed. The students tended to pull that desire to serve from different areas. First, students like Hannah, Mark, and Steve seemed to pull from a place of pride and allegiance to family and neighbors. Mark and Steve also described rural areas being entitled to the same type of quality healthcare 
and physicians as anywhere else. Dianne and Isaac, though, felt the desire to serve at a personal level, such as connecting or reconnecting with a rural population. For Dianne, she intimated seeing patients who reminded her of her family members and understood where they were coming from. Isaac reflected on how his position had changed, and was now in a place to help his community that he had left.

\section{Theme 3: Motivation to Stay: Family}

One of the most common themes that surfaced when discussing the opportunity to practice in the state or region was proximity to family. As already recounted in the Theme: Family, Community, \& Land Associations Remain Important, this theme resurfaced numerous times when discussing future practice opportunities. Megan, Isaac, Steve, and Dianne, all firstgeneration students, intimated family and being closer to home as a reason to practice in rural West Virginia. When asked what may be his biggest motivations to come back to his home county after medical school and residency, to which he replied, "my family," to which he described as being the hardest aspect to leave behind. When asked about barriers to leaving, he described still only living 30 minutes from his parents, who he explained were, "getting a little older." He then mentioned the prospect of leaving the state, but still coming back later to be closer to his parents. Dianne, who had left the state for another career, gave a similar response about family being a major motivator. She replied, "Well, staying close to my parents, my parents are elderly and they just they don't... that was one of the reasons I came back." Further, she explained that she would have regretted it if her parents passed away before she came back and spent more time with them. She concluded by stating, "it's been wonderful being able to be near my siblings and go visit them on a weekend... family is a huge consideration, too." 
Finally, when asked, Steve did not explicitly name family as a motivation to return, but he stated his desire to return home and work locally and the entirety of interview implied family as a major factor. He noted when asked about his family's relationship growing up, "I mean, essentially, because growing up rural,... my family is like everything to me, I mean... that's kind of like all I had,... you do everything together when you grow up on a farm.” He later stated when describing the possibility of working further away but still living in his hometown, "I'll commute if it's for... better benefits and a better contract, but ideally, I can just stay close to home and pretty much raise my kids the same way I was raised... just kind of keep the generations going."

Although many students spoke openly about either their desire to practice rurally or within the region, a number also coupled this discussion with taking their partner into consideration. Megan, Mark, Emma, and Leah all referred to their partner being from the state or region and how that may play into their decision for a final practice decision. For example, Megan felt a pull to return to her hometown after residency again noting her connection to a family and the farm. This feeling was amplified by also have a significant other from that same community noting:

Having my family nearby, it's really big for me... Maybe I would feel differently if my fiancé was from like a different area... that would probably play a part, but since we're both from here it's like almost double the kind of force.

Emma and her significant other are not from the same hometown, but he, too, is a medical student from Appalachia. When asked about her potential motivations to practice in West Virginia, she noted in part, "My family's here, ... I'm close with my family, so staying here and getting... to be around them." When asked about potential barriers to stay, or reasons to 
leave, she noted, "We're both really passionate about West Virginia and the population here, you know we're both very, kind of, proud of being from West Virginia" to which she followed up by explaining that they still may "want to experience something different, at least for a while."

Mark, who grew up in a more populated area of West Virginia, explained that he and his spouse were from the same community and although he hopes to practice in a rural area of West Virginia, he talked about taking his partner and potential future family into consideration. He noted:

I think if it was just me I would... live in, like the most rural place in West Virginia I possibly could, but... I have to think about my wife, we're probably going to start a family in the next few years, so... I think about all the opportunities, I had growing up. Similarly, Hannah illustrated her plans to practice in rural Appalachia and explained that it would be desirable to be closer to family, which is located in rural south-central West Virginia. However, her partner is originally from a major city and currently expects to commute (after the COVID-19 pandemic) to his job in a Washington, DC suburb. In this instance, her desire to be closer to home is moderated by her partner's career, to which they have opted to settle in the West Virginia’s Eastern Panhandle.

In short, the prospect of being close to home and family member was a theme that surfaced both explicitly and implicitly throughout interviews. Moreover, many students were actively considering the background, expectations, or needs of their significant others and how that may or may not impact where they choose to reside in the future. Subtly, though, as students enjoyed the prospects of being close to family back home, they also balanced the idea of potentially leaving, to which sometimes included a consideration of their departure as perhaps only temporary with the opportunity to come back. In fact, both Hannah and Dianne had already 
exercised that potential since they both left the state for extended periods to pursue a career before medicine, but now returned and planned future rural practice. Moreover, both saw family as a reason for staying in the state after medical school. However, the prospect of leaving coupled with the concept of being able to return is a notable tension throughout many of the interviews and is further explored and explicated in the next section.

\section{Theme 4: Motivation to Leave: The Prospect of Returning}

As prefaced in the previous section, many students proposed the idea that they may be able to leave, whether that be for residency training, post-residency training, or future practice, but still come back to the state or region. This notion surfaced, to some degree, in nine of the interviews. The only individual, Jared, who did not propose the idea had clearly stated he felt no Appalachian identity and had no desire to practice in the region. Additionally, Dianne and Hannah had both lived outside of Appalachia, both in major cities and Hannah outside of the country, between college and medical school. Dianne spoke of the previous city she had lived, her return, and reconnecting with population of patients during the third year required rural rotation, which she completed in West Virginia's Eastern Panhandle. "I just felt my roots coming back, I was like man,... I understand these people, because I grew up this way and I felt like I could connect with them better." Hannah did not speak about her transition back to West Virginia as openly as Dianne, but she did discuss an expectation by her grandmother to come home.

[My grandmother] was like, even when I went to college, 'she'll be back' like 'give her a few months, she'll drop out and she'll be back, because that's what West Virginia girl's do,' like 'they don't stay away from their moms.' 
Of the seven other students, all entertained the idea that they could leave the state or region, and it would still be there to come back to. Megan and Emma discussed the idea of returning to their hometown, mostly in the context to be close to family. Steve who is leaving the state and region for residency, plainly stated, "I'm going to return home to the farm, just because that's where I want to be.” However, the remaining four students discussed the idea of leaving and returning with knowledge or a new perspective. Isaac gave a detailed description of how he may leave and come back and how that would actually benefit the state.

The girl I'm... dating now, and myself, I've always wanted to go down to maybe the Carolinas. I don't think I'd have the courage to do it by myself, but I think... me and her, like because I've never really gone anywhere... [college] was the farthest I've lived... from home. So I never got to experience that... go a couple hours away and get away from it... but kind of find a similar population and maybe eventually because, her family's from [West Virginia],... maybe at some point come back up here but kind of be down there for the majority of my 30 s... And then also,... I went to [medical school] to bring medical knowledge to [my home county], I could go to North Carolina to bring different knowledge back... eventually.

Although not as descriptive as Isaac, Leah and Heidi who are leaving the state for residency, both described receiving advice from medical school faculty that they may benefit from training elsewhere, and possibly returning with a new perspective or outlook. Heidi noted:

As far as faculty, I had a couple people who are like, 'you know, you've been here for undergrad, now med school and, you grew up here, you need that experience so then if you decide you want to come back, you'll have different perspectives from all around.' 
Similarly, Leah explained, "I had advice from multiple attendings... that basically said... go and get training somewhere else and then... if you want to come back... you will have a place here." Mark discussed coming back as a sense of obligation to serve (described in theme two), but he also described knowing a rural physician who he saw as a role model. That individual left after residency, completed a fellowship at an Ivy League, and eventually returned, devoting their life to rural medicine. That definitely influenced me a little bit, maybe sort of just like maybe reaffirmed what I already knew or was hoping."

Throughout the conversations and discussions of what may lead someone to leave the state or region after medical school, many students who entertained the idea of leaving also entertained the idea of returning as a placating counterweight. Similarly students also described how if they either left the state or region, or ultimately decided to live or practice in a non-rural area, they may still be able to contribute to more rural areas. Heidi and Leah, both future psychiatrists, discussed the possibility of providing telemedicine to rural areas. Leah, who is leaving the state for residency, described feeling pulled to the state because of limited care opportunities for patients. She described the pandemic as changing her perspective on future possibilities with telemedicine. She explained:

[The] COVID [pandemic] has really shifted a lot of things online so even if I wasn't physically in the state I'm probably going to make sure I have a license to practice in West Virginia and then I can do... tele-psychiatry in the state to help with that need... I think there can be a multitude of reasons of why that might not happen... I think I... feel a pull strongly enough to be like well, I know that I can do my job virtually... that can still be very helpful for [psychiatry]. So, I think that that would be part of my career plan. 
Emma as well as Heidi discussed the prospect of rural outreach, such as traveling to rural clinics, for example, one day a week, or a couple days a month. Emma recalled a formative experience in medical school where she was able to fly with a physician to a southern West Virginia county to see patients. Heidi noted, similarly, of inspiring pediatric orthopedists who travel to rural West Virginia locations to see patients. After asking her if she is interested in practicing medicine in a rural area, she noted that rural practice was unlikely for personal reasons, but also because of the challenges to practice certain potential future subspecialities in a rural location. However, she noted, "I would love to do like an outreach type of clinic or... outreach in some type of way."

To summarize, many students see the prospects of leaving the state or region and are also able to recognize the potential to eventually return. Some even couch leaving as a way to bring back additional knowledge, skills, or perspectives to the patient population. The ability to return serves almost as an anesthetic for the prospect of leaving. Similarly, a few students were able point to other ways they may serve the patient population if they indeed do not return or practice in a non-rural area, which included discussion of telemedicine and rural outreach.

\section{Theme 5: Limited Direct Influence: High Achievement}

Each student was asked how their performance in medical school, such as metrics on high stakes exams, influenced or is influencing their decision to stay in the state and the region. In most instances, the student was also asked how they think it may have influenced their peers. Many participants downplayed the role that achieving higher metrics on United States Medical Licensure Examination Step-1 or Step-2 Clinical Knowledge examinations may have on motivating a student to look elsewhere for their residency training or future practice. ${ }^{23}$ In the

\footnotetext{
${ }^{23}$ The United States Medical Licensure Examination (USMLE) is divided into three phases or steps. All must be completed and passed for licensure. Step-1 is most commonly completed by students at US accredited allopathic medical schools after the basic science phase of their respective school's basic science curriculum. Step-2 Clinical Knowledge (CK) is completed during the clinical phase of the school's curriculum and Step-3
} 
majority of discussions, most students talked about the decision to leave as a personal factor that was not subdued by lower examination metrics or necessarily bolstered by higher metrics.

Simply, many students chalked up the decision to being more related to personal preference or finding a program for residency as a good personal fit, whether that was in the state, region or elsewhere. Many students simply seemed destined to stay or leave for a myriad of potential reasons. However, students recounted that metrics did have an impact on the type of specialty training or practice available to a student, which then may cause some students to consider other options elsewhere. However, as recounted below, there are nuances in how or where students believe it is possible to access those residency opportunities.

Isaac, Steve, Megan and Dianne, all first-generation Appalachian students, downplayed the role of metrics and talked about personal preference in both future practice and residency program selection. Steve, a future emergency medicine doctor, noted:

I don't know if [metrics have] any influence, because I know some people that just like to practice rural because they like it... and I know people that want to practice rural because they like it and they're an average.. medical student, and I know people who want to

\footnotetext{
is regularly completed during residency. All three phases must be passed for licensure. Step- 1 has given a three digit numerical score, but is currently moving to a pass-fail scoring system, which will formally become the standard in January 2022. All students in this study would have received a three-digit score. Step-2CK is also a three-digit score, but is not currently planned to be phased into a pass-fail system. Step-1, with the traditional three digit score, has historically played a major role in the residency match. Residency programs and specialties have commonly utilized the three digit score in the application process, and in recent years there has been a greater emphasis on earning a high score on Step- 1 to be more competitive in the match process. The term "match" or "matching" is the process and procedure that graduating allopathic medical students participate in to find a residency program. The National Resident Matching Program (NRMP) facilitates the majority of allopathic residency positions in the United States. Students typically apply to residency positions and undergo school visits and interviews in the fall before graduation. In the winter, students rank their top choice of residency specialty and program (location). Similarly, graduate medical education programs (residency programs) rank applicants. A computer algorithm at the NRMP "matches" students with programs. Outcomes of "the match" are announced nationally on the same day each March. In effect, students and residency programs are "matched" together (National Residency Matching Program, 2021).
} 
practice rural, who are extremely smart and some people like, why are they going to go practice rural? It's like well, rural people need good care, too.

Isaac, who matched to a family medicine program and denoted a Step-1 score approximately ten points above the national average, stated that he did not look into many other programs or options than the program he selected in West Virginia. "I kind of wonder... where could I have gone, what could I have done, but for what? I know I would've picked this place anyways." Megan, who matched to obstetrics and gynecology, explained that some programs at WVU such as pediatrics and family medicine have a rural scholar's program that allow a student to automatically match to their residency if they commit to the program early. Obstetrics and Gynecology is not on the menu of specialty options to be a rural scholar, but she explained, "Regardless of like my board scores or not, I think that would have been such an awesome program to have and do." Finally, Dianne noted that perhaps some students may want to achieve a certain specialty or have a desire to live elsewhere, but noted, "it seems a lot of my fellow students are from this state and are happy to stay nearby because their families are here, too." Mark wants to return to West Virginia after an obstetrics and gynecology residency but was noncommittal in his response.

Yes, and no, I mean, I guess I would say yes, and I feel like people that... are like high achieving, like high scores,... it's kind of like oh they're going to go to some big name place. Then of course they're unique examples of people who have grown up in West Virginia that just want to stay at WVU because they love WVU.

Emma also leaned toward the idea that the decision is a personal preference by noting: Maybe some people who stay, who might not have if they had better scores, I think that's few and far between, I think most people kind of go where they want to go, ultimately... 
but... I think... you definitely kind of see people shift... like, oh someone was going to do one thing, and... maybe they didn't get the scores to do that field... and now... they're doing primary care... someone who's the opposite... [is] like, oh, I was going to do, primary care and then I got like a super high score now and doing dermatology.

Several students also offered up the perception that a high score on Step-1 or Step-2 may not in and of itself necessarily change the mind of someone who had already found or decided upon their residency specialty or program location, but may be a factor in the type of residency they may be able to achieve, which had additional implications of where a student must go to obtain that residency. Mark noted that many students matriculate without being totally sure on their future practice specialty plans, but as he notes, "They really leave it up to... Step-1 at the end of the second year and kind of see how they go through the first two years and what their scores are." From there, students see themselves as divvied into different residency possibilities with the most prestigious, such as, for example, dermatology or neurosurgery at the most competitive end, but family medicine, pathology or psychiatry on the least competitive side. Emma and Heidi made similar statements; however, Heidi's comment speaks to a greater nuance between what high achievement may mean in the decision to leave. "The higher the score, more often you can do more competitive things, and... in that regard, you have to apply super broadly." Nonetheless, she concluded, "some people, though, even if they do get that high score, they want to stay."

Hannah, though, made a similar but ultimately conflicting statement. She noted that a high score coupled with a higher class quartile may position a student well to match into a competitive residency program. However, she followed up by denoting that actually very competitive students, such as those with top metrics and in the top quartile may be better 
prepared to match in a competitive specialty elsewhere compared to classmates, but may be less competitive against medical students from highly ranked medical schools with name recognition. In turn, those students are compelled to stay to more easily achieve their specialty choice.

Even as a most competitive student at WVU, you're going to be less likely to get that really competitive spot at... Johns Hopkins, so I think that the people, probably doing [dermatology], urology, orthopedic surgery, neurosurgery, they'll probably stay,... I think that they are probably ranking WVU pretty high because they think that they can get a spot at WVU.

Jared, a future obstetrician and gynecologist, considering a future fellowship in Reproductive Endocrinology and Infertility (REI), however, he explained that it is necessary to consider the downstream implications of residency practice, particularly if a student wants to seek a competitive fellowship. He recounted a number of qualities that drew him to his top choice for residency.

The chair's a reproductive endocrinologist, they matched an REI to Harvard two years ago, one of the other organizers is Harvard trained, you know,... those... pieces are really important... if you want to sub-specialize. Now if I wanted to be a generalist, I'd be totally fine finding a community program that has high case volume to get proper surgical training and so then I'd probably be more amenable to like staying at [a hospital in West Virginia] or going, you know, somewhere, maybe in-state, but to sub-specialize you really got to be strategic and looking at the faculty and looking at the historical fellowship match rates of the programs.

To Jared, seeking a competitive program elsewhere was important because of the need to network for a fellowship. 
In sum, for the majority if not all of the students in this sample, achieving certain metrics or class ranking did not resonate as a direct reason to ultimately choose a residency or future practice in a certain location. However, step scores and quartile rankings do ultimately help a student decide on their type of specialty, which may have implications for where they may be able to find a residency. The influence of scores on specialty as opposed to directly on location of practices creates more of an indirect impact. For some students, for example Isaac and Steve, their scores were seemingly not as influential on their desired practice location. However, metrics do determine the type of specialty that is available to student, which may have impacts on where they decide to seek residency training and ultimately practice. Higher metrics and performance may unlock the proverbial door to competitive residency programs elsewhere, but many students are not necessarily seeking to open and step through that door for personal reasons or personal preference. Moreover, Hannah and Jared present an additional nuance in that Hannah explains that the most competitive students are actually more likely to achieve their residency specialty at the school they graduated from, while Jared argues that for certain opportunities to subspecialize, it is imperative to seek out programs that allow for network building. The concept of specialization and sub-specialization and its influence on future practice opportunities is further explored in the next subsection.

\section{Theme 6: Tension: Career Implications and Perceptions}

Some students in the study recognized implications for their career by seeking a rural practice. For example, Leah and Heidi both described limited opportunities to participate in research or academic medicine if they were to practice in a rural setting. Others did describe practical considerations, like how their pay or benefits may be different. Dianne alluded to the idea that perhaps rural pay may be less, but ultimately noted, "but that's not a concern of mine,... 
I'm going to make plenty of money as a doctor, no matter where I'm at." Jared, similarly, did not see rural practice as a financial limitation even as he noted no desire to work in Appalachia. He explained that rural settings may allow for opportunities to make more money because of provider need. Conversely, though, Steve and Isaac, who are both interested in rural practice, did have financial concerns. Steve mentioned that he wanted to work at his hometown hospital, but worried the pay and benefits may be less than if he commuted to larger hospitals, which he considered as a possibility. For Isaac, when asked what he saw as limitations to return and work in his hometown, one factor he specifically mentioned was money, as such, how much money he would make in his hometown compared to elsewhere. To briefly summarize, although things like academic medicine, research, and financial opportunities were factors in students considering where they may someday practice, they were actually limited and mixed. However, as is argued in the rest of this theme, the prospect of specialty type and sub-specialization were major factors that students either saw as limiting in their ability to practice rurally, or actually gave them the opportunity to do so.

Students were asked how their desire for a certain specialty or to subspecialize after residency may impact their decision to stay or leave. For some students, specialization was a major factor when considering rural practice. In large part, some students discounted the prospect of rural practice because they believed that their specialty was not compatible with rural settings and may face substantial hurdles. First, some described the challenges of having a healthy work-life balance in a rural area. Mark described how he thought his specialty, obstetrics and gynecology, may be challenged by rural practice despite his desire for rural Appalachian practice. He noted that rural areas typically have fewer obstetricians/gynecologist and women's healthcare suffers in those areas. However, he coupled that with the notion that he likes the idea 
of being a single provider in a smaller community, but worried that this type of practice would cause strains on his work-life balance and his personal life. He relayed, "you have that... perceived notion of... I can't ever take off,... what if I'm not there and I'm the only person there to [provide care]." He mentioned that instead of working as a single provider in a rural community he may feel more compelled to join a group practice to help balance the workload. Jared, also a future obstetrician and gynecologist explained that this type of scenario put forth by Mark is why he will not practice rurally. Jared intimated that working in a rural area would keep him from becoming an REI, because of having too few patients (explained further below). He went on to say that an obstetrician/gynecologist, in a rural setting would have a generalist type of practice, and he stated, “...if I did generalist obstetrics and gynecology, I'm taking call every two or three days and... I'm unwilling to do that."

Emma, a future psychiatrist described the patient volume of working in a rural area due to limited mental health providers. She rhetorically asked, "how do I get as many patients in to see me as possible without overwhelming myself?" These comments largely speak to the concern that rural practice may have an impact on work-life balance. Steve, who is very set on returning to his rural family farm described being concerned with working alone. He is planning to work as an emergency medicine physician and denoted that there are a number of important benefits to working in larger hospitals, and providers have to be sure they are comfortable being without certain resource in a rural setting. He used the example that having other emergency room physicians on a shift at a major hospital is very different from being the only doctor working overnight at the rural hospital in his hometown. Because of this, he stated he specifically sought out residencies that provided rural training to be sure he was comfortable working with fewer resources, including other doctors. Dianne made a similar notion that through her residency 
training, she thought that would help her become comfortable being alone. However, she also noted feeling she had physician colleagues at other facilities and institutions she could rely on for advice and guidance if the need arose.

Although some students described being overworked, others saw working rurally an impossibility because of limited patient volume for certain specialties and sub-specializations. As prefaced above, Jared is considering reproductive endocrinology and infertility subspecialization, as is Megan. Both described how difficult a related practice would be in a rural setting. First, as recounted above, Jared explained that if you want to sub-specialize, you are likely already considering future fellowship opportunities when finding a residency. There is a need to network and find residencies as stepping stones for fellowships. He also mentioned that he did not see a reproductive endocrinology and infertility practice in a rural setting as practical. Megan also spoke specifically about sub-specializing in this field. She noted that it would be costly and it may be underutilized.

If I would want to specialize... there would be some hardships, I think... Whereas it would be nice to kind of bring that specialization to the area, but I don't know if it would necessarily thrive just with education level and... the value of it with money.

After that, she was asked what she meant by education level, to which she further gave an example of rural perceptions. She noted:

Some people don't... like birth control, because they just saw... someone posted on Facebook or their religious background forces them... [to] not want a kind of birth control, whereas they don't know there might be another kind of birth control. So, that's kind of... what I mean by that, it's just an example. Maybe... they don't know that REI 
even exists to help them have a baby, maybe they've been trying for years and they don't know that that's even a possibility, kind of thing.

Megan's comments present several considerations about rural practice. There are undertones of patient healthcare literacy challenges, such as understanding how to engage certain types of medicine, particularly reproductive medicine in a rural community. There is also the practical matter of having enough patients for a certain type of medicine. It could be a substantial financial risk to develop a highly specialized practice in a rural setting.

The concern of having patients and infrastructure was not limited to just obstetrics and gynecology. Heidi, a future pediatrician who wants to subspecialize in a field like cardiology, critical medicine, or hematology-oncology, also saw her sub-specialization choice as a limiting factor for future rural practice and denoted these types of specialties were often in academic or at least major medical centers.

Although some students particularly saw some specialties or sub-specializations as limiting their ability to practice rurally, several students instead saw certain specialties as opportunities for rural practice. Steve noted:

One of the main reasons I picked [emergency medicine] was because of the shift work, and I can still maintain a farm doing shift work... being a physician is great, but it's only half as important as farming to me.

One caveat that Steve offered, though, was that working as an emergency room physician in a rural area still has challenges, such as hospitals needing only a fewer doctors that may not turn over, which can lead to waiting longer periods of times for opportunities to find certain employment. Isaac also denoted he picked family medicine for the lifestyle benefits. He explained he could have the opportunity to operate his own clinic, which could give him 
flexibility in his personal life. He recounted his dad coaching little league baseball and wanting to be able to do the same types of things for his family.

Some students also gave examples of unique opportunities in rural settings not afforded in non-rural settings. First, as recounted, certain types of specialties do not lend well to rural settings because of patient limitations. However, Hannah, a future family medicine provider planning to practice rurally, provided a differing thought process. She described rural practice as opportunity to provide a broad scope of care. She recounted some of her mentors, explaining, “They'll just see somebody for COPD or their [arterial fibrillation] and they'll go... do a joint injection and then they'll go and do an aspiration of a boil... and then... take care of somebody... on the [ventilator]." She explained that medicine has gotten more specialized and actually, it is a skill to be less specialized, something she talked about admiring. For herself, she described seeking a one-year fellowship in dermatology for family medicine physicians. This opportunity would allow her to integrate some needed specialized care into rural primary care. Jared agreed with the notion that rural medicine allows for opportunities to broaden a physician's typical scope of practice. He explained that some rural doctors are able to own a practice, but moonlight at their local hospital in an intensive care unit, which he described is a way to increase salary in a rural setting or have different opportunities.

This theme presents a particularly interesting dichotomy between how students recognize rural healthcare needs and how they ultimately respond to them. In numerous interviews, students both explicitly and implicitly described or made note of Appalachian and rural healthcare limitations and provider shortages. Some students, as was particularly recounted in the theme regarding a desire to give back or serve, saw their practice as an opportunity to step into the breach and help fill a void. Some particularly chose their specialty for certain qualities 
including the opportunity to practice rurally. Finally, for Hannah and similarly recounted by Jared, rural practice provided an opportunity to engage more than one type of practice, which can have implications such as bringing certain specialties to primary care, the ability to manage a multitude of patients, and even practical implications like a higher salary. There is also the prospect of working alone, which perhaps at first seems like a negative, but can actually be a positive. For example, when Steve noted his concern for working alone, he also described that some students and physicians may be drawn to rural practice because they may more quickly become a leader.

At the same time, others saw the healthcare limitations of the region as further prohibiting and could impact a work-life balance. Mark and Jared are prime examples, such as the need to take call in a rural setting could have significant impacts on their personal life. Others saw the practice type as impractical or not feasible. Jared and Megan discussed reproductive endocrinology and deduced that patients in a rural setting may dismiss or misunderstand the care or simply have fewer resources to access the care. This is despite that fact that Megan, for example, recognized that there are patients in a rural community who may benefit from reproductive endocrinology. This was not relegated to just obstetrics/gynecology, as, for example, Heidi saw practices in pediatric cardiology as having limited opportunities in a rural setting. As noted, students commonly recognized healthcare shortages and limitations of the region and those limitations also served as a deterrent for practicing there. To summarize this point, Emma, a future psychiatrist, explained:

[The] lack of resources, that's... one of the reasons to stay but it's also... one of the things, like it can get frustrating at times to deal with... the lack of... support for certain things... If I maybe went to a state that, like have [sic] more resources for mental health 
care,... have better internet access,... like maybe I could do a lot of good there, and you know, maybe it would be easier for me, maybe selfishly, to want to go somewhere else and do those things and have those resources for my own practice.

\section{Theme 7: Motivation to Leave: Opportunities, Amenities, and Socialization Elsewhere}

A recurrent theme throughout the ten interviews were amenities or conveniences typically more common in more populous or at least less rural areas. First, it was not uncommon for students to discuss opportunities for future families. Several students talked about educational opportunities and the ability to send their children to quality schools. Isaac and Steve, who both talked about their rural upbringing and their desire to live rurally talked about challenges their family may face. Steve noted that he was fortunate to go to a private school despite his rural upbringing, and he fostered a great sense of internal motivation.

When you grow up rurally, yeah, you might be a success story for your town... being the only one who got out... but you always have that risk, you're worried that your kids might fall into the trap... you're just worried that your kids might not... end up on the same track you did.

Steve did note that, "you also have that risk in the city" and went on to explain that the right opportunities fell in place for him, but that does not mean the same type of opportunities will necessarily fall in line for his children, which is a factor for consideration. Isaac made a similar comment, he stated growing up that they had limited opportunities and he sensed a setback when it came to academics and competing in sports. He noted, "I would want... to give my family the greatest opportunities... knowing what I grew up with,... but again... no matter where you go, it's all about self-motivation... but I'd like to give them a good... starting shot... to kind of do what they could." 
Steve and Isaac were not the only students to describe educational opportunities for their family elsewhere and how that is an important consideration. Emma talked about being torn and wanting to help people in the state, but also followed up with rhetorically asking, "Do I want to experience something different?... Do I want to have a different experience for my kids?" Later, when asked what barriers may make it difficult to practice in state or in her hometown, she noted, "If I have my kids go to a school that has more resources than somewhere in Appalachia... then maybe they could have... more resources in their future as well." Heidi noted, "I think I would want more opportunity for... my kids and family, because at that point when you're about to practice, you are thinking like, okay, where's best for my family than more so just for me?" Mark, who noted a desire to practice in rural Appalachia, explained an internal debate about how that may impact his children's opportunities to participate in some of the opportunities that he did, such as sports and orchestra. He stated, "I think about those sorts of opportunities for my kids in the future, so... I don't want... my career, my lifestyle choices to have... a negative impact on my family."

Some students specifically talked about particular socialization opportunities or being able to access certain communities. There were an assortment of specific examples that some individuals described regarding opportunities for socialization or opportunities for interactions with others. For example, Hannah discussed her spouse having family and friends from outside of the US, which created significant difficulties when they or others traveled due to the lack of major airports in the region. Regarding more diversity in the region, Jared specifically noted, "not having a large LGBT community drives me insane... I don't like trying to search for other gay people to hang out with,... I would much rather have a much more solid community." He further noted that there were more established communities in cities and urban areas. Leah noted, 
"I could see people of color having issue,... that might be a barrier... I mean the state makeup is very predominantly one skin tone... I'm not saying, that is [a barrier], but I think it could be."

There were also particular amenities or conveniences that were described that students saw as a positive for living elsewhere. Jared, who adamantly stated he did not plan to return, was asked what his biggest motivations were to go elsewhere. He described both educational opportunities for a potential future family and impacts of rural practice on lifestyle preference (e.g., not wanting to be on call as an obstetrician/gynecologist in a rural community). But, he also explained things like opportunities to go out to restaurants, in general "things to do", and he noted, 'life experience,... I don't think you're able to experience as much whenever you're in a rural area." Similarly, Emma noted, "I'm not much of... like a big city person... but... maybe there's certain things I'm able to do there that I wouldn't have staying in West Virginia.” Leah was more specific, and noted nightlife as an example, but also described other opportunities. "Just like interesting things to do... just like walk out to your park and there'll be yoga in the park... if you live near a city or... even... a more well-funded state, those opportunities are there." Additionally, and of note, some students talked about wanting to live more closely to beaches and generally in a warmer climate.

Many of the students relayed certain opportunities, amenities, or ability to interact with others as a limitation to stay in the state or Appalachia. Foremost, many students, even those favoring rural living and practice, wondered how that may impact their children's ability to experience educational opportunities. Of note, Hannah provided a different perspective to this discussion. Having gone to private school in the state, she described West Virginia as actually having cheaper private school opportunities and that by staying in the state it may make it easier to provide that opportunity for her kids. Similarly, when asked about barriers to practice rurally, 
Dianne described that she would have once said the isolation and inability to have or find certain amenities. However, she stated the world is more connected now and used the example shipping groceries directly to her home. Dianne and Hannah also described indifference to some of the specific items that other students described, such as restaurants and opportunities for entertainment, like shows. Hannah specifically noted only having Applebee's, Outback Steakhouse, and Chili's in her current town, but noted it was still more than her hometown, that only had a Dairy Queen that is now closed. She noted these factors were not enough to live elsewhere. Hannah and Dianne did, instead, mention opportunities to recreate outside. An important note is that Dianne and Hannah had both spent a number of years in major cities, and in Hannah's case outside the country. Being non-traditional students who left rural Appalachia and came back for medical school, they may have less motivation to leave for amenities typically associated with more populous areas.

It was not uncommon in the interviews for students to delineate some amenities or socialization opportunities that may be limited by practicing in rural Appalachia. One of the most common perceived limitations were family opportunities, particularly as they related to education. This was prevalent for both students who desired to practice elsewhere, or who were considering practicing or living in the state or region. Also, some students described lacking diversity, and how it is a consideration for themselves or could be for others. Others simply discussed limitations for their personal lifestyle, like restaurants or nightlife. However, these opinions were not universal. For example, while some students valued restaurants, shows, and nightlife opportunities, others simply described those things as perhaps a luxury or they found personal fulfillment by being in nature. In sum, many of the aforementioned factors, particularly educational opportunities for family were an important consideration for students, regardless of 
their ultimate decision for practice location. However, they did not always see these factors as enough to ultimately decide to leave or to not return. In part, these were factors among many others, including some of the earliest relayed with this research question, that include considerations of rural lifestyle and proximity to family. These amenities, opportunities, and preferences were being weighed against those other factors. 


\section{CHAPTER VI: CONCLUSIONS}

\section{Study Overview}

This research leveraged qualitative case-study methodologies, including semi-structured interviewing of ten Appalachian medical students from one medical school. Sociological theories were utilized for analysis, which is delineated below. For the first two research questions including, how Appalachian students interpret their relationship with home as well as who or what serve as facilitators or impediments, Bourdieu's (1984) habitus and related capital theories help deconstruct and ultimately situate the findings within the already established literature (Maton, 2012; Moore, 2012). Moreover, the third research question, regarding motivations to stay or leave Appalachia, is better analyzed through the lens of Tönnies (1887/2001) theory of gemeinschaft and gesellschaft.

\section{Theoretical Analysis: Habitus \& Capital}

As Bourdieu proposed, an individual arrives at different situations or "games" with varied levels of understanding or knowledge (Maton, 2012; Thomson, 2012). This knowledge can include even knowing that the game exists, how to access the game, and that there are rules to play the game. The knowledge, skills, familiarity, or understanding comes in the form of cultural and social capital that is acquired through previous experiences and affiliations (Maton, 2012). How an individual perceives, understands, and engages experiences is a part of their habitus. The habitus is a culmination of their background and history, which is influenced by further factors including family, opportunities and choices of previous generations, culture, and society. 


\section{Upbringing, Family, \& Educational Opportunities}

By utilizing this theoretical framework, there is evidence that Appalachian students who successfully matriculate to medical school received positive educational endorsement throughout their upbringing and adolescence by family members, particularly parents. All students in this study recounted at least one parent, and most recounted both, as strongly advocating or expecting academic achievement. Aside from formal academic support, students recognized opportunities and experiences provided by their parents as making a difference in their academic trajectory. For example, Steve and Hannah both recounted private school as being life changing. More subtle exchanges or interactions-Dianne checking out scientific library books with her father, Steve being compensated for supplemental reading, Heidi's parents taking her to the Body Worlds Exhibit-were also profound and long-lasting. As has been recounted in the literature, family, and particularly parents, of Appalachian and rural students having positive or reinforcing viewpoints of education are important for the development of high achievement aspirations and educational self-efficacy (e.g., Ali \& Saunders, 2006; Byun, Meece, Irvin et al., 2012; Demi et al., 2010; Dyk \& Wilson, 1999; Hlinka, 2017; Hunley, 2015). These findings are further supported by this study.

There was not a consensus of either the mother or father being particularly more educationally supportive. Mark and Hannah, whose mothers had college degrees but fathers did not, described support being different between the parents with mothers particularly stressing academics. Megan, a first-generation student, also specifically reported aspects of her mother providing academic support growing up. Emma, Leah, and Heidi all had parents with at least college degrees or higher and saw their father stressing education more than their mother. Dianne, a first-generation student, identified her father as the main educational motivator. 
However, Isaac and Steve, first-generation students, and Jared, a continuing-generation student, did not necessarily recount one parent over the other as having higher expectations or being more or less motivating. These findings are as complicated as the literature surrounding the matter, which has come to disparate conclusions on which parent, and in what circumstances, a mother or father may be more educationally influential for rural or Appalachian students (e.g., Ali \& Saunders, 2006; Blackwell \& McLaughlin, 1999; Chenoweth \& Galliher, 2004; Dyk \& Wilson, 1999). Therefore, it is challenging to ascertain exactly and to what extent each parent influenced their student's educational trajectory in this study as well as draw a connection to the greater literature.

Some students described how their parents' employment or education had a normalizing effect when it came to the sciences or healthcare. For example, Megan's mother was a nurse and Emma's father worked in hospital information technology, which they saw as making hospital interactions comfortable and normal. Similarly, although not in medicine, Leah's parents had science oriented graduate degrees, which she noted as setting her on a path for a science field. Other examples were put forth in Chapter IV, but the argument is that parents projected high academic achievement as the norm. This norm was coupled with exposure to meaningful and commonplace interactions with the sciences and medicine, at times facilitated by simply having a parent who worked in or around healthcare. Endorsing high achievement and engaging in supplemental educational opportunities, particularly related to science and medicine, were all forms of cultural capital that parents fostered.

In retrospect, the argument of having parents who endorsed education or certain educational experiences for a positive outcome is perhaps not altogether unique considering the educational achievement of these students. However, it is important to recall that all students in 
the study are from Appalachia, mostly from rural communities, and six went to a low income high school. That is to say that parents and their academic endorsements were vital as students did not necessarily always feel that their community or schools prepared them well with educational opportunities. A number of students reflected that they could have benefited from more opportunities to prepare for higher education. Some recognized college peers from cities or outside of Appalachia as having more opportunity including high school college preparation courses. Dianne was perhaps the most critical of her education, claiming it was the reason she changed her college major and pursued another career. Heidi lamented not having access to a diverse range of career fields or access to members of the community who held a wide variety of careers. Several students, including Steve, Megan, Leah, and Dianne, saw limitations in their high school education and college preparation. For some of those students, they denoted how their experience was likely better than others in the region (such as having some AP course options), but still less than could be expected elsewhere, including in larger Appalachian cities. These findings are supported in the Appalachian literature which has documented a general feeling among college students that they were, in some ways, educationally disadvantaged or disserved (Bush, 2015; Holbrook, 2016; Powell, 2008).

Despite these notable educational challenges, students from Appalachia have also been shown to generate a strong internal locus of control and strong desire to succeed (Holbrook, 2016; Wallace \& Diekroger, 2000). The literature shows that rural students who have higher academic self-efficacy growing up ultimately have higher career expectations as well as educational aspirations (Meece et al., 2014; Wettersten et al., 2005). Five of the students, Jared, Hannah, Isaac, Steve, and Heidi, included themselves when asked who or what motivated them to do well in school. They described internal pressure and developing high personal expectations. 
Hannah and Steve provide some of the best examples of this self-motivation. Hannah noted, "I was always much harder on myself than my parents were on me." While Steve explained about college, "The only reason I did well is because I have a ton of internal motivation just to do as best as I can."

Reconsidering the concepts of habitus and capital, students saw limitations in their community's educational offerings and general opportunities, particularly as it relates to college preparation as well as career possibilities (e.g., Heidi noting she did not know or understand the concept of research). Considering the lens of habitus, if college were "the game," these students were not receiving all of the rules or skills to participate. Steve, perhaps, summarized it best when he noted that even though he went to a private school, he still had fewer opportunities to participate in college preparatory courses. Some students, in a similar scenario, cannot adapt even if they want to, because they do not have the skills to succeed in college. "They go to undergrad. and... get absolutely rocked by the curriculum and... fail out and then go back home and do some blue collar job, even though they wanted to go to college... That's definitely a barrier."

It is not to say that students were altogether unhappy with their education, in fact many recognized how they were in some way fortunate or lucky, because they knew of less privileged areas in Appalachia. While there were educational limitations, parents tended to fill voids. All students recognized positive reinforcement for doing well or keeping up with their academics and not disappointing their parents. This desire to do well was leveraged into a strong selfmotivation to be academically successful and make the most of opportunities. The desire to achieve for family also manifested in an identifiable strong self-motivation for high achievement. 


\section{Shifting Family Relationships}

When many of the students in this study transitioned to college, they did notice a change in relationships with parents. In fact, Isaac, Steve, Leah, and Heidi recounted growing closer to parents and valuing their relationship with home. Isaac and Hannah both particularly felt some emotional turmoil while in college, which seemed grounded in guilt. Guilt and internal conflict are common feeling for Appalachian college students who leave home or devote time to higher education (Bryan \& Simmons, 2009; Welch, 2013). Hannah was worried about not being there to aid her grandparents, one of which was ailing. Isaac struggled with the idea of leaving his parents describing them as "getting older." Similarly, Dianne noted that her ability to return for medical school after a stint in another career ameliorated some feelings of guilt stating that she would have been "really sorry" if she did not come home and spent more time with her parents and they were to pass away.

Demi et al. (2010) explains that for rural students, having a stronger relationship with parents is not necessarily a great indicator when it comes to determining educational aspiration and achievement. However, when rural students do have a strong relationship or hold higher admiration for their parents, they may feel compelled to ultimately try to live close to their family (Johnson et al., 2002). Both factors seem to be at play in these findings. A number of students, particularly those recounted in this section, discussed a favorable relationship with parents that grew stronger when they actually went away to college. Moreover, all the students in this section recounted ways that they would like or would consider staying closer with their family when they practice medicine. The theme and concept of staying close to family is further explored and connected to the literature in the section utilizing theory of gemeinschaft/gesellschaft below. However, students in this study looked favorably on their 
family and parents and were generally concerned with leaving them, which actually manifested in a closer relationship.

As it relates to habitus, students were brought up in an environment that endorsed and rewarded academic success. In many respects, students, in turn, fostered a great deal of academic motivation from their parents and valued their family relationships. That is to say, parents presented ideas and opportunities, both academic and extracurricular, and students recognized these opportunities. College became obligatory or an obvious next step. Considering habitus, students recognized parents as vital to their success, and putting them on their academic trajectory. Parents had fostered and cultivated ideals of academic achievement, and introduced and provided tools for the game. However, by accessing higher education (i.e., the game) these students ultimately had to move away from their family, which was challenging for some. Isaac provides a helpful example, by noting, "[My parents] got me to where I was and I just wanted to take advantage of the opportunities I had." He also noted feeling "distraught” when he would leave home after visiting during a weekend from college. As students gained motivation and inspiration to be successful, they ultimately had to leave these same individuals, which was not always easy.

\section{Seeking Medical School Guidance: Parents \& Family}

Students in this study tended to arrive at the idea of medical school on their own, but at times with the help of media, including television. As Mark noted, "I think most of it just came out of like... sort of the standard... path for, you know, you're good at science and want to help people kind of a thing." Isaac explained he decided on medicine in college in part because, "The possibility that I could." Others like Jared and Heidi drew inspiration from television, including both educational and scripted dramas. Heidi also felt a limited understanding of potential careers 
and pathways related to health and science. She stated, "I didn't know... what research was until I left [southern West Virginia] ... or, like all those different opportunities within the science field." Taken with the findings above that students were educationally supported and provided with supplemental science material, students generated a proclivity for science and healthcare. However, students typically arrived at medicine because it seemed to make sense and they believed they were academically able.

Although parents were academically supportive and encouraging, a limitation for Appalachian medical students is actual family understanding of medical education. When approaching medical school, some students sensed questioning from family that ranged from simply inquiring about length of study and cost to more general derision. Most of the students who reported questioning did not necessarily couch it as outright discouragement, but instead, family wanting the student to consider all options and make an informed decision. These types of interactions seemed to occur most frequently with women and first-generation students. Dianne, and Isaac, both first-generation students, and Heidi a continuing generation student, all noted that their parents questioned the medical education length and cost or offered alternative career routes like becoming a nurse or physician assistant. Hannah recounted questioning from her grandmother who she noted as having traditional gender viewpoints regarding women and their priority in the home and as family caretaker. Megan, a first-generation student, described some generally derisive comments and attitudes from extended family including sarcasm.

Generally, the literature shows that Appalachian and rural women often feel pulled to pursue careers that have gender associations, such as nursing (Beasley, 2011), and may be more applicable to working in the local community (Blackwell \& McLaughlin, 1999). Moreover, Appalachian women have also been found to receive more dissuasion than men when it comes to 
going to college (Wallace \& Diekroger, 2000). Regarding Hannah, specifically, rural women may be expected and ultimately feel pressured to avoid personal aspirations, like college, to instead fulfill gender expectations and stay in the home (Agger et al, 2018; Donaldson, 1986; Dyk \& Wilson, 1999; Meece et al., 2014; Snyder, 2007; Welch, 2013).

Gender aside, it is also possible that this questioning may be coming from a place of general uncomfortably with engaging such a high level of education. Some literature has shown that parents of rural and first-generation Appalachian students are often uncomfortable with the college environment (Bryan \& Simmons, 2009; Hunley, 2015; Snyder 2007). Of note, though, one distinction is that this literature reflects college at the undergraduate level as opposed to medicine or professional education. The students in this study saw parents as supportive for college, but they were more likely to experience questioning or lack of useful parental guidance related to the decision to enroll in medical school. These findings help expand the literature by including medical education and the role that parents play in helping Appalachian students consider medical school. Considering habitus and the discussion of parent's educational influence above, these students paid particular attention to opinion of their parents as they credited them for much of the early opportunities and motivations. Therefore, parents having a questioning stance is likely all the more confounding for students when they placed high valuation on their expectations. Investigating the basis for this type of questioning is an area of the literature that needs expanded.

\section{Seeking Medical School Guidance: Mentors and Physicians}

Although some students did have parents who were in healthcare, none were physicians and no students recounted having a relative who was a doctor - the closest were Hannah and Megan with mothers in nursing. Many of the students did recount positive impressions and 
interactions with their pediatricians; however, only Steve had a mentor/mentee relationship with a childhood doctor. Later, when students did formally access physicians for shadowing and medicine exposure, some were actually discouraged by the attitudes exuded from the physicians. These interactions can be impactful for some students. Hannah opted to try another career before committing to medical school because of the negativity. Positive interactions with physicians or mentors that can provide level setting expectations and guidance was seen as invaluable by the student sample, particularly considering that there were limited inherent physician family mentors. For example, Leah, whose parents both had master's degrees in science fields, denoted: I almost wish like I did have someone in my family who was or... like I knew who was a physician, just so they could be like, "Yeah,... I know school hasn't been hard for you before, but like this is hard."

Although not a physician, Steve cited a college professor providing such guidance. He explained that this professor reiterated that medical school would be a challenge and he should not expect to be the top of his class. Steve leveraged this advice and performed well on his first exams in medical school, which gave him confidence and momentum to keep studying and doing well.

Some students, particularly Megan and Emma, explained a connection with medicine or a hospital through their parents and employment while Steve, through childhood illness, established longstanding relationships with pediatricians. However, most students were unable to recount associations with physicians that were outside of the physician/patient relationship. This lack of social capital was more evident when students were asked if anyone in their life dissuaded or deterred them from medicine. A number of students indicated direct dissuasion or at least disheartening conversations before medical school including during shadowing experiences but also during casual community interactions (e.g., Jared at his father's business). Shadowing 
experiences are particularly important for students as it helps gain exposure to various types of medicine and a variety of career options as well as ensure students have fully considered the medical vocation, such as seeing the field from the working side and not just the academic.

Shadowing experiences also allow students to access working physicians who can become mentors. Heidi discussed how shadowing an orthopedist turned into a positive mentor/mentee relationship throughout medical school and the doctor found ways to help expose her to other aspects of medicine, such as research. She ultimately decided on pediatrics, but felt this physician was a central figure in her medical education journey. However, these types of valued experiences were coupled with accounts from others who characterized physicians as discouraging or dismissive. These interactions came at a time when students were actively inquisitive and impressionable about their career path. Experiences ranged from physicians making light of student's interest in medicine to admonitions and dissuasion. Some students were able to balance those experiences with positive and encouraging interactions with other doctors. Nonetheless, negativity toward the field can have lasting impacts, such as for Hannah who decided to pursue another career citing physician dissuasion as a prominent factor.

The literature shows that in a rural setting, the volume of physicians is often impacted by and impacts the number of students who ultimately pursue medicine from that area (Anderson et al., 2009; Wheat et al., 2015). Community programs to expose rural adolescents and students to medicine are of particular importance in a rural setting where there are already fewer physicians in that environment (Anderson et al., 2009; Crump et al., 2014; Gross et al., 2012). Firstgeneration students and students from low-SES backgrounds often have difficulty engaging physicians outside of the typical patient-physician relationship (Bassett et al., 2018; Bassett et al., 2019; Brosnan et al., 2016), which is also true for Appalachian students. Several students 
noted the implication for how they would have benefited from having a doctor in the family. Steve, because of childhood illness, was the only student to form an ongoing relationship with a physician while growing up. His contact with that physician and subsequent doctors for treatment became a point of aspiration. However, other students often faced discouragement or negativity about medicine. This study aligns with a point made by Southgate et al. (2015) who argued that low-SES students may have aspirations to pursue medicine, and they may even have the metrics and academic performance to do so; however, there are still challenges in how to actually engage medical school.

A major impetus for this study was physician shortage in Appalachia; therefore, it stands to reason that aspiring students would likely have more generally limited personal relationships with physicians. The most common interactions were simply seeing a pediatrician, which were more often recounted as positive but not particularly meaningful. Other experiences, including shadowing, were broad and the physicians had vastly different attitudes about medicine. Students were left to parse out what they saw as beneficial and realistic information versus personal opinion that may be jaded by specialty or practice challenges. Thus, as students have fewer natural medical engagement points, they also have to decipher mixed messaging from physicians. It is not to say that students in the study only wanted positive or reinforcing information, because that did not seem to be the case. However, advice was hard to interpret already, and these seemingly reliable points of access were further confounding.

Limited access points to engage the medical community make positive parental and family influence all the more vital. However, as parents are able to endorse academic achievement during upbringing, they are less prepared to guide students to medical school. At times their own questions or concerns about the intensive educational experience add another 
layer for students to consider, which is complicating in a culture that values family harmony and cohesion. There are already fewer physicians in the region, with even fewer in rural areas making it a challenge for families and students to draw personal connections (i.e., social capital). When interactions with physicians do occur, they are more meaningful, even when they are negative. For families, expecting their child to do well and attend college is one thing, but medical school is another. It is not to say students are unsupported, but for parents there are greater risks, such as length and intensity of study, reliance on family resources, and substantial debt. When students were questioned, the length of time and cost were coupled with alternative healthcare fields. Appalachian students have a close relationship with their parents and their opinion is worthy of their consideration, which makes gathering their input all the more important. These issues have the potential to compound and serve as impediments to the pursuits of medicine.

\section{Available Cultural \& Social Capital}

Although students arrive at medical school with limited capital for medicine, they are also uniquely equipped with cultural and social capital for certain aspects of the clinical realm. Students move from the basic science to the clinical phase of the curriculum around the third year of medical school. In the rural and community experiences, students are able to leverage both cultural and social capital to optimize their experiences. For the students who went back to their home community, some were able to directly reconnect with people they knew or were able to establish connections based upon community or family associations. Even for students who did not go back to their hometown, students still felt at least an understanding of their patient population. Appalachian students are well positioned to engage this patient population, even if they ultimately did not see themselves practicing rurally. 
The literature has found that medical students with low-SES and first-generation backgrounds are able to leverage and utilize cultural traits and understanding to better connect and care for patients (Brosnan et al., 2016; Conway-Hicks \& de Groot, 2019; Southgate et al., 2017). This study contributes to and expands this literature as Appalachian medical students are also able to employ their own cultural understanding and associations to engage this unique patient population. Numerous examples can be relayed including Isaac stating that he could connect with patients and the problems of his home community. 'I've seen them, I've been around it my whole life, someone who knows their way around a hay bale type of stuff." Steve recalled how on his rural rotation he met people who reminded him of his parents, and they have the same mannerisms which it comes to seeking care, such as, waiting to do so. A final example includes Dianne noting, "When they find out that you're from the same roots... they're trusting."

The literature has argued that having a rural background increase one's likelihood to practice rurally (e.g., Brooks et al., 2002; Herd et al., 2016; Isaac, et al., 2014; Jones et al., 2009; Jones et al., 2014). Other literature shows that rural rotations and experiences can increase the interest in practicing rurally (e.g., Crump et al., 2016; Crump et al., 2019; Playford et al., 2017; Walker et al., 2012). However, how much or to what degree rural exposure and experience can change or shape a student's practice trajectory is debatable. As Brooks et al. (2002) posited, it is a nature versus nurture question. Because students are able to connect with cultural associations via rural experiences, this study aligns with other literature that rural background may be critical for future rural practice, but rural experiences can buttress and support a student's potential to do so while they are engaged in the educational process (Kondalsamy-Chennakesavan et al., 2015; Playford et al., 2017; Walker et al., 2012). This study does, in many respects, support these notions. The majority of students were from or affiliated with rural Appalachian counties and 
towns and some explained how rural rotations did confirm or increase their desire to practice rurally. More specifically, though, the students in this sample were able to connect with, understand, and more readily identify with the patient population, which may have been more challenging had they grown up elsewhere. Therefore, these findings do lend credence to the idea that rural background and experiences are important factors when it comes to being comfortable to practice with a cultural patient population.

Along with being notably comfortable working with this patient population, students also recounted physicians during medical school that exemplified or were identifiable as role modeling some aspect of rural living or rural practice. Identifying these physicians was clarifying for some students that they could exist in two ideals simultaneously. For example, students could live or practice rurally but they also could have a multifaceted and specialized medical career. Taken together, rural experiences and rural physician influences are important when considering the entire continuum of the Appalachian medical student's experience.

The literature shows that students from non-traditional backgrounds (including low-SES) may have difficulty connecting with medical school faculty in part because of lacking commonalities, such as experiences in their upbringings and class associations (Nicholson, 2013). Moreover, first-generation students in medicine have been shown to avoid engaging faculty, particularly for help, being concerned they may be punished or looked down upon (Bassett et al., 2018). However, Appalachian students in this study did not necessarily recount difficulty with engaging and interacting with medical school faculty. In fact, it was the opposite as students found role models who became particularly important both in the main teaching hospitals and in the rural settings. This was in large part due to cultural associations and affirmations that as a student from Appalachia, there were physicians in medicine who were like 
them. Examples include Dianne, Hannah, Isaac, Mark, and Steve who all met physicians who promoted a rural lifestyle or practice and were well-respected with a decorated medical or academic career. As Steve noted, "I definitely feel like a type of connection with them whenever I find out that they have a farm and they're a physician."

The five students that this finding was most evident were students from families with the lowest educational attainment. Dianne, Isaac, and Steve are first-generation students while Mark and Hannah had fathers with high school diplomas and mothers with college degrees (Hannah's mother a doctorate). They also had some of the strongest desires to practice or live rurally with Dianne, Isaac, Mark, and Hannah all planning to practice rurally while Steve was undecided about practicing rurally but planned to at least live rurally. There also seems to be some connections between the gender of the student and the physician as the three males recounted male physicians who were influential and the two female students identified with female physicians. Dianne noted about rural practice and her role model, "She got me interested... it kind of gave me perspective of... you can... practice in West Virginia and do a lot of good work." Hannah and Dianne are both non-traditional students and received some level of questioning from their family when considering medicine. With the literature above in mind (Bassett et al., 2018; Nicholson, 2013), identifying and connecting with faculty mentors with a rural association are important as they exemplify the potential to practice rurally and provide levels of affirmation.

In sum, many students relayed or described healthcare disparities in the region and they typically identified as Appalachian. The community/rural experiences were an opportunity to begin reengaging a culture they had participated in and for the most part, had a longstanding identification. Additionally, physicians in the medical school environment both in rural and 
academic settings showed students a way forward for future practice possibilities, including exemplifying rural lifestyle or practice. In other words, students did not always have the ideal cultural and social capital before medical school, such as direct access to physicians, but once in medical school they brought important cultural capital to re-engage the Appalachian patient population. Some students ultimately saw themselves working rurally in Appalachia because of these experiences. Similarly, students crossed paths with doctors who they identified with and ultimately fostered a new sense of potential. Albeit, not all new relationships led students to desire rural practice, in fact, for students like Heidi, some faculty encouraged her to leave for other experience. Nonetheless, students like Mark, Steve, Isaac, Hannah, and Dianne identified role models, which they saw as in some way positively contributing to or framing what it would mean to be a rural doctor in Appalachia.

\section{First-Generation Students}

Given that Appalachia suffers from notable educational limitations and under attainment of college education, college generation status was specifically considered when recruiting for this study. As such, four participants in this study, Megan, Steve, Dianne, and Isaac, are firstgeneration college students. Megan's mother had post-secondary training to become a nurse and Dianne and Isaac both had fathers who attended some college but did not finish. Steve's mother completed a two year degree. Of the four students, Megan, Dianne, and Isaac attended a lowincome public high school, while Steve attended a private Catholic high school. The students went to four different colleges, two of which are public universities in West Virginia, one is a private institution in West Virginia, and one is a public university outside of West Virginia.

The literature argues that college typically becomes somewhat of an expectation for students from middleclass backgrounds (Bradley, 2017; Reay, 1998). However, all students in 
this study, first-generation and otherwise, recounted a college trajectory from an early age and explained that their parents stressed education. As Dianne noted, "It just was always understood that I would go to school." There is considerable evidence that findings from this group support much of the argument put forth by Gofen (2009) regarding the benefits of family capital for firstgeneration students. Among other things, parents of successful first-generation students make education a priority, regularly discuss and stoke aspiration, normalize educational engagement, promote family solidarity with emphasis on the children's future, and establish sibling role modeling (Gofen, 2009). There are a number of examples that can be relayed that support these concepts. Steve being compensated for reading and good grades as well as crediting his parents for making sacrifices to send him to private school are prime examples. Similarly, he and Isaac both talked about not wanting to disappoint their parents and wanting to make them proud. Another example is Megan commenting that her parents pushed education since they themselves did not go to college. Moreover, she described all the help she received from her mother to do well academically. Similarly, Dianne recounted weekly trips with her dad to the library and his encouragement when she struggled in college. The literature shows that parental involvement and the development of educational aspirations is important for all students, not just firstgeneration students (McCarron \& Inkelas, 2006). However, first-generation students commonly have lower aspirations and encouragement and support is particularly important for this population (Lohfink \& Paulson, 2005; Pike \& Kuh, 2005).

Patfield et al. (2020) has argued that aspiration of first-generation students is leveraged through three methods: the projected familial habitus, such as college as a means for future potential; meritocratic familial habitus, which leverages hard-work, dedication, and accountability; and, the supportive familial habitus, which hinges on academic encouragement. 
All three categories could be discussed with this sample of students, but the meritocratic familial habitus is distinguished here (Patfield et al., 2020). Concepts such as students referencing ideals of determination, hard work, perseverance, or resilience are common themes among the firstgeneration literature and this study contributes to that conversation (e.g., Alvarado et al., 2017; Lehmann, 2009; Luzeckyj et al., 2017; O’Shea \& Delahunty, 2019; Tate et al., 2015). Lehman referred to first-generation students as commonly holding a "moral advantage" because of their life experiences (Lehmann, 2009, p. 639). Steve explained about going to college that he had "a ton of internal motivation" to do well spending lots of time in the library and stating, "I definitely... had to earn my way to get to medical school." Dianne recounted deciding to go back to school for medicine and taking post-baccalaureate classes. She recounted working her "butt off' in her math course. When she received some resistance to going into medicine, she recalled, "I want to be a doctor, that's what I've always wanted to be as a child... I'm not going to back down. I thought, I'm going to do it, I'm going to go all the way." Isaac, when describing his high school, noted, "You had to be self-motivated and a lot of people that... came from a different family background weren't self-motivated and they did not take advantage of... furthering their education." He followed up by commenting that education was a way to "get out."

The first-generation literature has shown that students often struggle with the reformation or reconsideration of identity in college (Covarrubias \& Fryberg, 2015; Hsiao, 1992; McInnis \& James, 1995; Reay, 1998; Robert \& Rosenwald, 2001) in part due to what London (1989, p. 144) referred to as "biographical or social dislocation." Gardner and Holley (2011) further claimed that with more education, disconnects with family and home can intensify, including lack of understanding for why a student would seek advanced training. Neither Megan nor Dianne related identity crises or disconnects with their home and family. Dianne remained close with her 
family as she has done her entire life and Megan concluded that she may have had a more challenging transition to college if she has gone further away-her college was 90 minutes from home. Steve and Isaac both noted getting closer to their parents while in college, while Isaac did struggle to be away at times, particularly when returning to college.

When starting medical school, none of the students noted feeling a displacement; however, Dianne, Isaac, and Megan did recount questioning from family. For Dianne and Isaac, part of this questioning was related to the cost and length of training required, which somewhat aligns with Gardner and Holley (2011) who found that first-generation PhD students felt their parents did not always understand why they would pursue advanced education and questioned its practicality. A difference is that for Dianne and Isaac, the questioning was more related the length of the program and cost. For Dianne, it was suggested she become a physician assistant. This line of questioning and thinking, though, is not totally specific to first-generation, which was discussed above regarding the Appalachian rural literature.

There were connotations in the first-generation student's interviews related to how their social standing may change by going to college and medical school. Part of this related to mobility and agency to pursue different options, including leaving their hometown. The literature shows that parents of first-generation students commonly see college as a means to protect or enhance employability or security—such as, college as a practical means (Delong, 2003; Gardner and Holley, 2011; Lehman, 2009; Roberts \& Rosenwald, 2011; Thomas \& Quinn, 2009). The literature relating to first-generation, low-SES, and working-class medical students has found these groups commonly reflection upon and consider their own social backgrounds while in medical school (e.g., Beagan, 2005; Bassett et al., 2018; Brosnan et al., 2016; Southgate et al., 2017). Southgate et al. (2017, para. 72) noted students utilize a "tactical incorporation" when 
forming their medical and medical school identity, while Brosnan et al. (2016, p. 848) noted students leverage a "dual identity." In part, concepts like tactical incorporation and managing dual identities surface from medical students beginning to recognize their own social standing while in medical school (Bassett, et al., 2018). Moreover, there is an inherent classism in medical school where middle class norms and expectations are the most highly valued and dominant (Beagan, 2005).

The findings here closely align to this literature. A number of students discussed in some way how their social standing or connections had changed or were changing. Although there are examples from non-first-generation students, this theme was most prominent among firstgeneration students. For example, Steve also described education as being utilized by his family to advance social standing:

You know my mom was... really lower class, didn't have any money. And then now our family's middle class, and then you know, by the time I'm up and have my career... I'll be like upper-middle... or higher-class just based on opportunities I have, income, and you know things like that.

Megan, when asked who motivated her to go to college, explained her parents and particularly her mother. "I think, since they didn't go to college you know, they were very kind of almost, they weren't like pushy about it, but definitely like wanted us to go.” Isaac explained,

There was just not much to do in [my hometown], you go fishing, you go hunting... that's enough for a lot of people, but I wanted to go do other things and I wanted to see other things.... knowing what I grew up with... I'd like to give [my family] a good... starting shot. 
Regarding a dual identity, Steve and Isaac shared a number of similar characteristics and viewpoints, both being first-generation, growing up on family farms, and holding favorable views of returning home. The idea of returning home was balanced with the recognition of being able to "get out" while also distancing themselves from notable regional stereotypes. Steve was more specific making comments about his movement to upper middle-class as recounted above. He also explained, "Yeah, you might be a success story for your town... being the only one who got out" but he concluded that does not mean his kids would necessarily be able to do the same if he returned home. Similarly, Isaac noted, "The opportunity is there to get out, but you just have to know that's what you want to do and work for it and not just let it pass you by."

Megan also balanced the pros and cons of going back to her hometown as opposed to going elsewhere, and how she may view her hometown differently now that she will be a physician. "I still have a lot of friends, even back at home, who didn't go to college... but maybe it would be kind of nicer... to live in an area with... similar... thinking people I guess." In sum, students in this study, particularly those from backgrounds of lower educational attainment, recognized their own class movement, especially now that they were about to become a medical doctor. Moreover, this concept relates to the section below and theoretical analysis with gemeinschaft and gesellschaft, which describes how and where students foresee their future considering their upbringing, background, and social connections versus potentials for future practice elsewhere.

In sum, this sample of first-generation students is relatively small; however, given the educational context of Appalachia, it was important to ensure this generational status was necessarily included. All of the students recognized the potential for college early and saw it as an expectation, which is aligned with middle class ideas of college. There was a strong 
endorsement by parents to do well academically and on achievement in general, which is notable for enhancing aspiration. The students also offered a utilitarian viewpoint of education, in that college would be necessary for improving or securing social position. Finally, although some students sensed a changing relationship with home or family when going to college, which is common in the literature, students did not necessarily recount identity crises. However, several were balancing the idea of leaving their home community or "getting out" with what it may be like to return home in the future.

\section{Habitus Limitations and Future Research}

A limiting aspect of the habitus theory for this study is that this group of students did go to medical school and are thus academic high achievers. From this theoretical perspective, it is possible to make deductions regarding the characteristics, experiences, and opportunities students saw or recognized as limiting or facilitating their educational journey. These students were able to perceive and take advantage of the opportunities that led them to medicine and to be academically successful. As Leah astutely pointed out during her interview, "I'm going to be an MD in a month or two, so I really can't think that I missed out on too much or like I didn't have opportunities.” A future study should consider earlier perspectives using habitus. How do Appalachian high school students considering medicine, particularly academic high achievers, perceive their experiences and opportunities? The students in this study are afforded a retrospective look of how they made it to and through medical school, but a sample of precollege students actively considering medicine may or may not recognize the same barriers and support systems.

An additional perspective that merits inquiry is why some high achieving Appalachian students fail to aspire to medicine or related fields. The former paragraph asks how early aspiring 
students perceive a way forward in light of their surrounding and opportunities, but this question ask what limitations exist in Appalachia that keep young high achievers from pursuing a medical career. Six of the students knew from an early age they wanted to be a physician and many were able to recount specific meaningful interactions with science and medicine. Jared knew he wanted to be a physician from watching Beverly Hills, 90210, but later waivered and took extra time to decide if he should instead pursue the family business. Leah, Isaac, and Mark arrived at their decision mostly through a process of elimination, such as liking or being good at the sciences or enjoying aspects of helping or serving others. Considering these four students, Jared, Leah, Isaac, and Mark, it is evident that some students who are academic high achievers may not be on a clear trajectory to medicine despite having the academic potential. This line of inquiry can and should be expanded to other professional trajectories. However, of utmost importance for medicine, there is a provider shortage in Appalachia, which specifically impacts healthcare and access. The literature shows that there are relationships between the volume of existing providers in a rural community and volume of medical students from a community (Anderson et al., 2009; Wheat et al., 2015). Moreover, rural students who choose to pursue medicine have been found to do so from a relatively early age (Anderson et al., 2009). Therefore, there is a practical and pressing need to further the inquiry related to how students perceive and react to opportunities for the medical field.

\section{Theoretical Analysis: Gemeinschaft and Gesellschaft}

Research question three sought to explore how students perceive motivations and barriers to stay in Appalachia and particularly rural locations versus going elsewhere to live and practice medicine. It is best to first consider the sociological theory of gemeinschaft and gesellschaft put forth by Tönnies (1887/2001). This theoretical perspective relays two competing rationalities as 
it relates to how individuals perceive opportunity. Gemeinschaft is the concept that close interpersonal relationships are highly valued and are a necessity for building up the common good. Family, extended family, and close social connections, particularly in an area or region, are important and central to life. Tönnies (1887/2001, p. 27) explains, "Community by blood, indicating primal unity of existence, develops more specifically into community of place, which is expressed first of all as living in close proximity to one another."

Conversely, gesellschaft is a connection to society at large. Individuals lead a private life and participate in the greater society through contractual agreements. Interactions are more formal and based upon the notion that when something is given, something is returned and those interactions are impersonal, individualistic, and self-serving. In this way, greater society is composed of many contractual interactions. Those interactions are impartial to who or where they occur. Tönnies (1887/2001, p. 52) further described:

There are no activities taking place which are derived from an a priori and predetermined unity and which therefore express the will and spirit of this unity through any individual who performs them. Nothing happens in Gesellschaft that is more important for the individual's wider group than it is for himself.

This dichotomy is of particular consideration as students feel pulled to stay and interact with community and culture that they know versus assessing if they should leave for other opportunities. There have already been countless examples of gemeinschaft relayed, particularly in Chapter IV, such as close relationships with extended family, generational ties, connections to farms and places, and emotional struggles when leaving family for college. However, there are fewer examples of gesellschaft. Gesellschaft is much more easily identified when students discussed looking ahead, after graduation, and to their future careers. On one side is the prospect 
of traditional rural life, proximity to family, and desire to serve in the state or regiongemeinschaft. On the other are aspects of personal achievement, future career prospects, recognized agency and ability to leave, as well as non-rural lifestyle or medical practice preferences_-gesellschaft. These competing dynamics were evident throughout the collected data. As Tönnies recognized (1887/2001, p. 52), "In Gemeinschaft they stay together in spite of everything that separates them; in Gesellschaft they remain separate in spite of everything that unites them."

\section{Gemeinschaft}

Family, Identity, and Giving Back. Jared explained that he did not see himself as Appalachian and some other students who did identify as Appalachian also offered caveats to that identity. However, the majority of students did, in fact, recognize themselves as Appalachian or specifically West Virginian. A common refrain was that their identification was associated with family longevity in West Virginia or the region. That enduring association served as an important cornerstone for their own identity. Moreover, when students recounted their upbringing, it was common to explain family connections including extended family proximity. In a number of instances students denoted growing up on ancestral property, such as a family farm, and the volume of family who lived nearby. With gemeinschaft, an individual is strongly tied to a community because of the established interpersonal relationships that exist there. Those relationships include and are strongly reinforced by deep kinship ties that are interwoven, central, and reinforcing. How students in this study explained their identity, upbringing, and community ties speaks to the concept of gemeinschaft-close-knit communities tied tougher through kinship associations. There is an aspect of simply being a part of the community because 
of birthright and family association as well as a duty to persist and contribute to the upkeep of community networks (Stockdale \& Haartsen, 2018).

Several themes presented in Chapter V speak to the concept of gemeinschaft. First, a number of students recounted some of the challenges with leaving family for college. For example, Hannah explained feeling guilty being away from her aging grandparents and Isaac explained that he would be "distraught" when he would go back to college often thinking about his aging parents. However, many of the students actually formed stronger family ties by the time they graduated. Considering the concepts of gemeinschaft and the importance of interpersonal relationships, this may not be surprising. These students were away from family for the first time and while at college they became reflective on those ties and relationships wanting to maintain and strengthen bonds.

Gemeinschaft can also be utilized to better analyze how students perceive their options and opportunities for future practice. First, a number of students described wanting to give back or serve a community or the state. Some expressions included feeling an obligation, a sense of responsibility, not forgetting where they came from, and wanting to make a difference. These examples show how some students felt personally compelled to contribute to individual communities, the state, or region. Additionally, there were particular aspects of living rurally that resonated and remained important considerations such as doing so meant a form of independence. Some students described the desire to have few or distanced neighbors and favored a private lifestyle. Favoring rural characteristics was coupled with the idea of wanting to remain close to family. In fact, when asked why a student may want to stay in the state or region to practice, being close to family was one of the most frequent reasons given. Family proximity is particularly important when considering the overall idea that Appalachian students measure 
their identification with the culture through family lineage and association. In other words, there is a need to specifically weigh out what leaving may mean to a cultural association with Appalachia.

The literature has largely confirmed that students who have either a rural identity or a rural background are more likely to practice rural medicine (e.g., Brooks et al., 2002; Crump et al., 2016; Jones et al., 2014; Playford et al., 2017; Puddey et al., 2014). This study tends to support that notion; however, there are other nuances at play including a desire to live a rural lifestyle, wanting to serve or give back, and maintain family ties. Regarding rural lifestyle, Owen et al. (2007) found that medical students who self-identified as growing up rurally was more predictive than an individual's actual home address or school address being classified as rural. This predictability would indicate some accepted association with living in a rural area. The students in this study who were particularly pulled toward rural practice recounted ways that they identified with or were connected to either rural lifestyle or practice. For example, Hannah discussed simply wanting to avoid an HOA. Steve noted, "Being a physician is great, but it's only half as important as farming to me.” Additionally, some students (e.g., Dianne, Hannah, Isaac, Mark, and Steve) explained that rural physicians throughout their medical journey solidified rural practice as a possibility. Isaac recounted one role model who was known to live a rural lifestyle. " $[\mathrm{He}]$ is a guy that heavily influenced me to really look at what I wanted to do... he showed me opportunities." These doctors allowed students to realize that it was possible to be a highly trained physician and also enjoy aspects of rural life.

The literature also shows that students who are interested in rural practice are more likely to be orientated towards finding ways to serve, particularly their home community (Roseamelia et al., 2014). Similarly, among West Virginia medical students, students planning to provide care 
for underserved populations can be a positive predictor of eventual rural practice (Shannon \& Jackson, 2015). The findings of this study support this literature. Particularly, seven of the medical students denoted or described the possibility of staying in the state or region as a way to serve and give back to the people. As recounted in Chapter V, many students feel compelled to help their community or state. Several examples and quotes were relayed in previous chapters, but two notable examples include Hannah, who stated, "I feel like we feel a sense of responsibility to making [West Virginia] a better place" and Mark, "I just feel obligated to serve the state."

Strong feelings of connection and the desire to give back is also directly related to the stayers versus leavers literature and high achievement. The literature shows that high achievers from rural areas, including in Appalachia, are not necessarily dissatisfied with their hometown or upbringing, and that adolescents and young adults that are the most unhappy are also the ones least likely to have the means to leave (Byun, Meece, Irvin et al., 2012; Howley et al., 1997; Johnson et al., 2002; Petrin et al., 2011; Petrin et al., 2014). Similarly, most students were not inherently dissatisfied with their hometown and necessarily seeking a means to escape (although the idea of getting out did surface, as argued above). However, a notable tension with the findings and the literature is that a number of students did recount aspects of their community or culture they disassociated from. Examples include Steve recounting his identification as Appalachian, but not the "hunting, fishing, wearing camo" aspects or Isaac explaining that his childhood and college friends now "ride around on their side-by-sides" every weekend. These undertones of Appalachian stereotypes are important considerations in this study. The literature shows that cultural stereotype avoidance has been found to be a motivating factor to be successful academically as well as a motivating factor to leave the culture or region altogether 
(Gallagher, 2019; Hunley, 2015; Towers, 2005; Welch, 2013). At numerous points in this study, students recounted how they may or may not identify with some aspects of culture, which at times hedged on stereotypes. These students were not necessarily leaving to escape stereotypes, but avoiding stereotypes and certain cultural associations, even if they stayed, were evident considerations.

In sum, family and local community remains a constant consideration for this sample of students. First, as relayed above, this student sample saw their parents as academically supportive throughout their childhood and into college, and had generally positive perceptions of their relationships with parents. Moreover, there are specific aspects of the literature that support the argument that students would feel drawn to be close to their family. First, returning to the concept of social closure, rural communities are composed of groups of people with particularly strong relationships (Bjarnason \& Thorlindsson, 2006; Byun, Meece, \& Irvin, 2012; Coleman, 1988, 1995; Singh \& Dika, 2003). Collectivism, strong family bonds and connections through curated generational relationships are notable characteristics in Appalachian culture (Barcus \& Brunn, 2009; Beasley, 2011; Gore et al., 2011; Leon \& Hoffman, 2016; Tang \& Russ, 2007). Barcus and Brunn (2009) particularly found that in Appalachia, the decision of where to live (such as proximity to family and home) is more dependent on quality of local relationships and less about place attachment. Therefore, generational and family associations are engrained and this sample feeling compelled to considering going or at least stay relatively close is supported by the literature. Moreover, this argument connects to those above regarding student perceptions of wanting to give back to the community and overall feeling a positive connection to their home community. 


\section{Gesellschaft}

Primary versus Specialty Practice. As students transitioned to the end of the medical school education, they begin balancing ideas that align with gesellschaft. Gesellschaft does not value close community associations nor is there necessarily a duty to participate or maintain certain connections. Associations are not maintained out of social obligation or commitment, but are personal and often based upon individual preference. A constant consideration for students in this study was how their individual residency program or specialty of care would influence their future practice location.

Four students in this study are pursuing primary care, and three of those students planned to eventually practice rurally, while one did not. For the one student, Heidi, the desire to pursue a pediatric subspecialty was a barrier to practice rurally. Therefore, her residency was in a primary care field, but she planned to eventually subspecialize. For the remaining six students who were seeking a non-primary care field, one of those students, Jared, did not plan to practice rurally, four were unsure, and one did plan to practice rurally. Jared and Megan were both concerned that reproductive endocrinology, a subspecialty of obstetrics and gynecology, would not be possible or would be limited in a rural setting. Conversely, Hannah who plans to practice rurally and in primary care saw rural practice as an opportunity to expand practice possibilities and provide a broad range of care. She discussed a potential fellowship in dermatology geared toward family medicine doctors. Similarly, Isaac is also planning to practice rurally and in family medicine and discussed the potential to do a fellowship in sports medicine out of the region and bring that expertise back.

Shannon and Jackson (2015) argued that among West Virginians, those seeking to work in primary care also had a greater likelihood of practicing rurally. Of primary care specialties 
(family medicine, internal medicine, and pediatrics) some have found that students seeking family medicine are much more likely to practice in a rural setting, even compared to other primary care specialties (Rabinowitz et al., 2012; Roseamelia et al., 2014). Similarly, three studies found that among students, those wanting to work as generalist had greater interest in rural practice (Jones et al., 2009; Puddey et al., 2014; Walker et al., 2012). This study does not necessarily support nor rebut the findings and arguments from others.

Instead, taking the above examples together and the literature, the concepts of primary care, generalist versus specialization, and sub-specialization are much more nuanced when considering the individual trajectory of a single student. A number of students in this study are pursuing a non-primary care field and still considering rural practice. Others see specialization, especially sub-specialization, as more limiting in a rural setting. Heidi, training in the primary care field of pediatrics and later subspecializing in cardiology or hematology, believes these fields are better suited in a major medical center. Hannah and Isaac, both seeking primary care fields, are thinking about pursuing fellowships that they expect to eventually benefit and broaden their rural practice. Primary care versus specialization/sub-specialization is not a binary or dichotomous consideration, and moreover, the implication to follow any certain specialty may not necessarily lead a student to or away from rural medicine. Modern medicine has tended to favor specialization of practice. Hannah, for example, explained that working rurally allows for a broad spectrum of practice, but she, too, is considering a fellowship in dermatology for family medicine physicians to compliment her practice. All students are tasked with considering how they see their future career and specialty fitting into potential places they may or may not work and live. Students must balance considerations like working in their home community or near family versus what they see for their medical career. 
The questions for future research should move away from dualistic considerations of primary versus non-primary care or generalist versus specialist and their implications for future practice location, but should instead be more specific. Future research needs to investigate specific fields of medicine and ask what challenges or opportunities are perceived by students and trainees as they are deciding their next steps. For example, how does the expanding opportunity to complete fellowships as a family medicine physician change how a student sees practice opportunity in rural practice? How do emergent and expanding hospital systems in Appalachia change the opportunity for specialist to practice and live in rural areas? In future research, there is less of a need to ascertain how major groupings of physicians see rural medicine, but instead consider how specific fields see potential or challenges in those same areas.

Career Opportunities \& Characteristics of Rural Practice. Another consideration for students was how a career specialization in a rural setting may impact their personal life. Emma, a future psychiatrist, for example, noted the challenges and shortages of mental health care providers in West Virginia and Appalachia. She also wondered how she would manage large patient volumes without "overwhelming” herself. Similarly, Mark and Jared, future obstetricians and gynecologists presented similar questions. Mark was concerned with the volume and amount of on-call duty he would have and if would feel like he could ever truly take time off from his practice. Jared specifically noted that taking frequent call was one reason why he would not practice rurally. Of particular note, Emma and Mark, both discussed feeling some sort of obligation or responsibility to help the people and state, but they also worried about what committing to work in rural Appalachia may mean for their personal life. Steve, who adamantly 
wanted to return to his family farm was also concerned about some aspects of rural practice. He noted concerns with working alone and being the only physician on shift at a time.

When students in this sample were considering their options for the future and if they would ultimately go back to their home community, they were also considering the economical aspect and what that would mean for their future income and career growth. For some students, like Dianne and Jared, money was not a factor to work rurally. However, others, like Isaac and Steve, were concerned with what practicing in rural Appalachia would mean for their future income. Steve wondered how challenging it may be to find a job. Notably, both students are first-generation and desire to practice rurally. Heidi and Leah were concerned that the decision to be rural doctors would limit their academic career.

There was also the worry that working rurally may mean working alone or in isolation. Students, such as Steve, Emma, and Mark, described how their specialty and working in a rural practice may be challenging due to the isolation. Mark and Emma were particularly worried about being overloaded in their practice, while Steve was concerned about working alone in the emergency room. As recounted above, Megan also made mention that by going back to her hometown, it would still be nice to be able to socialize with "similar... thinking people." After probing what she meant and if she was thinking about a medical community, she noted, "Yeah, I mean that's definitely a factor."

Many of these findings align with the literature. Some studies have shown that rural experience in medical school may highlight rural isolation, particularly as it relates to socialization (Caygill et al., 2017; Roseamelia et al., 2014; Somporn et al., 2018; Walker et al., 2012). Moreover, considering Steve's concern that he may be the only emergency medicine doctor on shift, other literature has pointed to the fact that a community having limited medical 
infrastructure in place, including having fewer or limited physicians already, was a bigger factor in physicians practicing in that location than community makeup, including general SES

(McGrail et al., 2017). Moreover, he was also concerned that because of lack of turnover, he may not even be able to work at his hometown hospital. Steve's worry was coupled with others who worried that an academic career would be out of the question at a rural hospital. These concerns are also documented in the literature. Roseamelia et al. (2014, p. 265) recounted general anxiety that rural students may have, noting "the perceived lack of jobs and professional opportunities may deter them from future rural practice."

Finally, the literature has also shown that women doctors are more worried about "professional isolation" than men (Spenny \& Ellsbury, 2000, p. 183). A few women in this study did recount similar worries. Megan was concerned about professional socialization or networking while Emma and Heidi were concerned about limiting their academic and professional careers. An interesting caveat to these findings is Dianne, both non-traditional and first-generation, who noted that she was not worried about her salary expecting to make what she saw as enough money rurally and felt she had colleagues elsewhere in the state she could look to for advice and guidance as needed. She also explained that she was given a lot of autonomy in her previous career, which made her feel comfortable working alone and being in charge of others.

An important point is that many students, such as Mark, Emma, and Steve, intimated a desire to give back and serve. They also saw ways that they could contribute. But these thoughts were often coupled with the notion that these disparities are multifaced and run deep in communities. Taking up practice in rural Appalachia comes with its own set of challenges. At the same time, although there are abundant opportunities to serve and contribute to solving 
healthcare disparities like provider shortages, there were additional risks or challenges, such as managing a work-life balance, potential specialization limitations, and even working alone. The potential to serve and contribute is evident for this student group, but so are the risks and challenges, which presents a specific example of the contrast between gemeinschaft and gesellschaft.

Personal Amenities and Family Opportunities: Finally, some also wondered what living rurally or practicing rurally may mean for their personal lives and the opportunities of their family. Of course, a number of students made note of how living elsewhere would provide more amenities, which ranged from things like access to shows and plays, different restaurants, airports, and actually even types of healthcare facilities for family. Students were also largely concerned with the educational and extracurricular opportunities for future children. As argued, a number of students in this study lamented limitations in their schooling, but commonly, they also believed that they had some fortunate opportunities, had good family support, and importantly, they were self-motivated. However, students considered how or if staying in the state and region and perhaps living rurally may limit the opportunities for their children. Emma succinctly noted, "If I have my kids go to a school that has more resources than somewhere in Appalachia... then maybe they could have... more resources in their future as well." The prospect of what opportunities may or may not be available for the student's family downstream was a major consideration. Students balanced the notion of their own upbringing and staying close to family with the prospect of what may give their children the best opportunity for success, and how their personal preferences and decisions for future practice may impact those opportunities. Steve, who felt a strong pull to return to his family farm noted, "You're just worried that your kids might not... end up on the same track you did." Mark considered opportunities for his family in a 
rural setting and noted, "I don't want... my career, my lifestyle choices, to have like a negative impact on my family."

Alternative Ways to Give Back: When considering leaving, it was common for students to bring about ways that they could still contribute to the region. First, there was the idea that if they left, they could still return. Leaving could be a temporary decision, it was not necessarily a finality. In fact, some students talked about physicians they knew who left and came back or physicians who encouraged them to train elsewhere after medical school and then decide if they would return. As Mark noted after describing one physician's decision to return, “Anyone can come back to West Virginia." In some instances, students, like Hannah and Isaac thinking about fellowships, actually rationalized that leaving would be a benefit to the state or their community, in that they could return with more specialized knowledge. Along with the prospect of leaving and returning was the potential that a student could still contribute to rural areas in their practice. For example, students discussed telemedicine as well as outreach trips to rural Appalachia. Not all of these discussions were shaped around the idea of leaving Appalachia altogether, but instead, some noted that they may not return to their hometown, but they were still considering ways in they could contribute to areas that have healthcare needs. Students often rationalized the thought of leaving with seemingly placating caveats, such as the possibility of leaving being temporary, leaving could actually benefit the state or region (e.g., returning with different knowledge or perspectives), or the potential to contribute to the region's needs with telemedicine or rural outreach.

\section{Conclusions}

Considering gemeinschaft and gesellschaft, Appalachian medical students must weigh how family relationships and social relationships may change by not going back to their home 
community. Gemeinschaft is particularly important for this population as being from Appalachia includes an identity that is tied to generational persistence and continuity in the region. Conversely, as presented in the literature review, medical school presents an environment that values state-of-the-art science and technology, often favor population centers, and is partial to specialization (Crump \& Fricker, 2015; Rosenblatt, 2010). Appalachian medical students have completed a challenging and extensive educational experience and are eligible for a multitude of opportunities upon graduation. All the while, these students originated in a region with significantly fewer college graduates and much fewer opportunities to connect with physicians who could serve as role models. After residency, these nascent doctors are positioned to be high income earners, well above the average income of the region. Students have the agency, mobility, and ability to leave the state and region altogether. However, the decision is complicated. Family, community networks, and the ability to give back and serve those networks are on one side, while the other includes specialty and sub-specialization opportunities, personal lifestyle preferences and amenities, as well as family opportunities. Students have to balance these possibilities and perceptions. Notable techniques include keeping open the opportunity to return, leveraging telemedicine, and participating in rural outreach.

\section{Gemeinschaft \& Gesellschaft: Future Research}

There are several routes for future research considering gemeinschaft and gesellschaft and Appalachian medical students. This project considers students who are about to begin residency. How do perceptions change throughout residency? Only one student mentioned having a child and instead most described opportunities and prospects for a future family. Considering this population values strong family and generational ties, how would actually having children change the perception of where they would like to raise their family? When 
Appalachian physicians have children, do they feel a stronger pull to once again be near family or does the concern to find and provide high quality educational experiences increase?

Additionally, for students who are considering telemedicine and rural outreach, how many actually follow through in their career? Do students truly feel compelled to find creative or nontraditional ways to contribute to rural communities or do those feelings dissipate once they have settled into their career? Gemeinschaft and gesellschaft is a useful theoretical concept for understanding the decisions and perspective of Appalachian medical students as they look forward to their career. It can also be leveraged to better understand how perceptions, insights, and plans change as physicians' transition through the rest of their medical practice.

\section{Implications for Practice}

This study supports previous findings that students with a rural background or upbringing may be well positioned and ultimately feel inclined to practice in a rural setting. Schools that have the ability and resources to recruit from rural areas may serve two purposes that include improving access for students to go to medical school as well as the potential to enhance the number of physicians who may return to practice in a rural setting, such as a hometown. However, students may be ill-prepared or less academically competitive when beginning college due to factors like fewer AP courses or generally weaker high school academics. Therefore, college pre-medicine programming and pipeline programming should consider how to target and help students remediate academic deficiencies and engage educational opportunities not always available through their school system.

The more pressing matter, though, is guiding students to a variety of mentors. These mentors should necessarily include physicians who have a rural background and should be available to college students seeking shadowing experiences. As argued, an obstacle for rural 
students is to parse out useful information and advice from shadowing experiences and interactions with physicians. It seems as though students gain a fair amount of aspiration for medicine early, even when resources are limited locally. However, there is a need to gain reassurance from someone in the field while facing questioning family members, a four-year intensive academic commitment, and taking on hundreds of thousands of dollars in student loans. Moreover, along with reassurance there is a benefit to have someone who can be trusted to relay practical advice that is not necessarily specialty specific. Returning to the point above, mentors necessarily need to have a rural background. As shown in this study, students are able to connect with rural physicians and better understand how both their career goals and lifestyle goals can be accomplished. Therefore, the sooner that students can interact with physicians who exemplify either a rural lifestyle or rural medicine, the sooner they may recognize that rural medicine is a viable option for their career. As students begin engaging certain physicians to explore specialties even before medical school, they must be given the opportunity to explore what it would mean to live and work rurally.

Finally, rural and community rotations are particularly valuable in Appalachia. Related to concept of mentors, again these are experiences that allow the student to connect with physicians in their home cultural environment. Moreover, these are often the first time since starting medical school, and perhaps since going to college, that students reengage the Appalachian culture. These experiences come about in the clinical phase of the curriculum when students are firming up their career goals and residency plans. These experiences allow student to reconsider their hometowns and home culture in that process. For example, several students made note about connecting with the patients, thinking about the rural need for physicians, and the potential to serve. It can be a grounding experience. This study supports the notion that these experiences 
are vital not just for rural recruitment, but also helping students confirm or reaffirm what they plan to do with their career. In some instances, that may actually mean rural medicine is not the right path for them. Nonetheless, the experience still provides an opportunity to explore that possibility, and ultimately present the learner with an appreciation for the characteristics and challenges of rural medicine and rural patients.

\section{Final Conclusions}

This research project was initially sparked from three concepts. First, West Virginia and Appalachia suffer from a number of deep-seated and wicked socioeconomic challenges, including healthcare disparities and a healthcare provider shortage (Marshall et al., 2017). Second, West Virginia, in the heart of Appalachia, trains per capita more medical students than any other state in the country (AAMC, 2019b). Many of those students were born and raised in Appalachia and specifically West Virginia (AAMC, 2019b). Third, and finally, West Virginia ranks 35 th per capita for number of students who stay in the state in which they were medically trained (AAMC, 2019b). There are multiple angles and questions that could be raised from these data points, but the purpose of this project was to understand the transition and associated challenges that students from Appalachia experience while moving through the medical education experience.

This study seeks to contribute to the literature ways in which aspiration are formed and supported for medical school in Appalachia. Utilizing habitus shows that Appalachian medical students have difficulty accessing important social and cultural capital. A number of students in this study saw local educational opportunities, particularly for college preparation, as lacking. However, this thought was often coupled with how there are areas of the state or region with even fewer opportunities, and they were somehow lucky. Moreover, students often found 
academic support and aspiration in their parents, particularly from a young age. All students had parents who showed strong support for academics and educational attainment. Parents were often credited with providing educational material about the sciences and the human body, which ultimately stoked aspirations for medicine. Along with lacking educational opportunities in many of their hometowns, few students could recount meaningful mentoring relationships with physicians. When students did engage physicians, they were often met with mixed messaging. Moreover, there was even some questioning from parents when it came to the pursuit of medical school, including length and cost when their seemed to be other suitable career opportunities.

In many ways this study is about movement. As students prepare to graduate from medical school, there is the potential to consider their own class movement, particularly for those from families with lower educational attainment. Students from these families, and not necessarily just first-generation students, reflected on their changing social standing most openly. This study sought to keep educational attainment of the family as a forefront consideration. By doing so, it was possible to draw comparisons between students from differing backgrounds. However, family educational attainment, particularly in Appalachia, is not binary but instead occurs on a spectrum. Even students who are first-generation college students have parents who may have not completed high school up to a plethora of postsecondary experiences that do not necessarily manifest into a four-year college degree. Moreover, continuing generation students may have a mix of parental educational experiences of varying levels of academic achievement. This study has contributed to the literature by being inclusive of and considering differing educational backgrounds, but there is still a need in the literature to specifically study firstgeneration medical students exclusively, particularly from Appalachia. 
Finally, this study is also about geographical movement, which includes students leaving their hometown for good or potentially the Appalachian region entirely. Tönnies’ (2001) gemeinschaft/gesellschaft is a beneficial theoretical concept for this study. Appalachian medical students are caught between ideas and ideals of family, generational associations, and future contributions. Those forces are coupled with thoughts of the future, including new agency, a medical career, and the ability to provide opportunities for families. Ideas or dreams of working hard to get out are confounded by desires to nurture those established family networks and take up a rural lifestyle. However, as the data above shows, many students are pulled or drift away from the state and region. This study contributes a medical education perspective to the conversation surrounding stayers versus leavers. Medicine is an important field to study in this section of the literature because it requires considerations of high student achievement with the ubiquitous need for physicians in rural communities and in particular Appalachia. Students in this study were set upon a high achieving trajectory by their parents and did just that, achieved a very high level of education. They are ultimately left with the decision of what to do with and where to take that education. 


\section{References}

Afeli, S. A., Houchins, T. A., Jackson, N. S., \& Montoya, J. (2018). First generation college students demographic, socio-economic status, academic experience, successes, and challenges at pharmacy schools in the United States. Currents in Pharmacy Teaching and Learning, 10(3), 307-315. https://doi.org/10.1016/j.cptl.2017.11.016

Agger, C., Meece, J., \& Byun, S. (2018). The influences of family and place on rural adolescents' aspirations and post-secondary enrollment. Journal of Youth and Adolescence, 47(12), 2554-2568. https://doi.org/10.1007/s10964-018-0893-7

Ali, S. R. \& Saunders, J. L. (2006). College expectations of rural Appalachian youth: An exploration of social cognitive career theory factors. The Career Development Quarterly, $55,38-51$.

Ali, S. R. \& Saunders, J. L. (2008). The career aspirations of rural Appalachian high school students. Journal of Career Assessment, 1-17. https://doi.org/10.1177/1069072708328897

Alvarado, A., Spatariu, A., B. \& Woodbury, C. (2017). Resilience \& emotional intelligence between first generation college students and non-first generation college students. Focus on College, Universities, \& School, 11(1), 1-11.

American Academy of Family Physicians. (2020). Primary care. https://www.aafp.org/about/policies/all/primary-care.html

Anderson, D. M., Whitler, E. T., Johnson, A. O., Elam, C. L. Wilson, E. \& Asher, L. M. (2009). Increasing the medical school applicant pool: A key to training more rural physicians. Kentucky Medical Association Journal, 107, 55-360. 
Appalachian Regional Commission [ARC]. (2018 July). College Completion Rates in Appalachia, 2012-2016. Retrieved from https://www.arc.gov/reports/custom_report.asp?REPORT_ID=72

Appalachian Regional Commission [ARC]. (2019). County Economic Status in Appalachia, FY 2020. Retrieved from https://www.arc.gov/reports/custom_report.asp?REPORT_ID=76

Appalachian Regional Commission [ARC]. (2020). Education in Appalachia. Retrieved from https://www.arc.gov/wp-content/uploads/2020/07/DataSnapshot-

\section{EducationInAppalachia.pdf}

Appalachian Regional Commission [ARC]. (n.d.a.). Appalachian Region Health Disparities and Bright Spots. Retrieved from https://www.arc.gov/report/appalachian-region-healthdisparities-and- bright-spots/

Appalachian Regional Commission [ARC]. (n.d.b.). The Appalachian Region. Retrieved from https://www.arc.gov/appalachian_region/TheAppalachianRegion.asp

Arnold, T. G. P. (2017). Expectations, Choices, and Lessons Learned: The Experience of Rural, Appalachian, Upward Bound Graduates [Doctoral dissertation, Marshall University]. ProQuest Dissertations \& Theses Global.

Aschbacher, P. R., Li, E., \& Roth, E. (2010). Is science me? High school students' identities, participation and aspirations in science, engineering, and medicine. Journal of Research in Science Teaching, 47(5), 564-582.

Association of American Medical Colleges [AAMC]. (2017). AMCAS 2017: New Questions. Retrieved from https://aamcorange.global.ssl.fastly.net/production/media/filer public/7d/4c/7d4c5b3c-be52-4c93b382-8dcc9ea7ffd3/2018 amcas new questions- fact sheet.pdf 
Association of American Medical Colleges [AAMC]. (2019a). 2019 Report of Residents.

Washington, DC. Retrieved from https://www.aamc.org/data-reports/studentsresidents/report/report-residents

Association of American Medical Colleges [AAMC]. (2019b). 2019 State Physician Workforce Data Report. Washington, DC. Retrieved from https://store.aamc.org/downloadable/download/sample/sample_id/305/

Association of American Medical Colleges [AAMC]. (n.d.). Summary of MCAT Total and Section Scores. Washington, DC. Retrieved from https://aamcorange.global.ssl.fastly.net/production/media/filer_public/be/28/be28698f-3195-4db09377-ef95d5377217/mcat_total_and_section_score_percentile_ranks_2019_for_web.pdf

Atherton, M. (2014). Academic Preparedness of First-Generation College Students: Different Perspectives. Journal of College Student Development, 55(8), 824-829.

Baker, P. G., Dalton, L, \& Walker, J. (2003). Rural general practitioner preceptors-how can effect undergraduate teaching be supported or improved. The International Electronic Journal of Rural and Remote Health Research, 1-11.

Banks-Santilli, L. (2014). First-generation College Students and Their Pursuit of the American Dream. Journal of Case Studies in Education, 5, 1-32.

Barcus, H. R. \& Brunn, S. D. (2009). Toward a typology of mobility and place attachment in rural America. Journal of Appalachian Studies, 15(1/2), 26-

48. http://www.jstor.com/stable/41446817

Barrett, F. A., Lipsky, M. S., Lutfiyya, M. N. (2011). The impact of rural training experiences on medical students: A critical review. Academic Medicine, 86, 259-263. https://doi.org/10.1097/ACM.0b013e3182046387 
Barry, L. M., Hudley, C., Kelly, M. \& Cho, S. (2009). Differences in self-reported disclosure of college experiences by first-generation college student status. Adolescence, 44 (173), 5568.

Bassett, A., Brosnan, C., Southgate, E, \& Lempp, H. (2018). Transitional journeys into, and through medical education for First-in-Family (FiF) students: A qualitative interview study. BMC Medical Education 18(1), p. 1-12.

Bassett, A., Brosnan, C., Southgate, E. \& Lempp, H. (2019). The experiences of medical students from First-in-Family (FiF) university backgrounds: a Bourdieusian perspective from one English medical school. Research in Post-Compulsory Education, 24(4), 331355.

Beagan, B. (2005). Everyday classism in medical school: Experiencing marginality and resistance. Medical Education. 39 (8): 777-784.

Beasley, S. E. (2011). Country Roads Take Me?... An Ethnographic Casey Study of College Pathways Among Rural, First-Generation Students [Doctoral dissertation, Boston College]. ProQuest Dissertations \& Theses Global.

Beattie, I. R., \& Thiele, M. (2016). Connecting in class?: College class size and inequality in academic social capital. The Journal of Higher Education, 87(3), 332362. https://doi.org/10.1353/jhe.2016.0017

Bejarano, C. \& Velverde, M. (2012). From the fields to the university: Charting educational access and success for farmworkers students using a community cultural wealth framework. Journal of the Association of Mexican American Educators, 6(2), 22-29. 
Benson, R., Heagney, M., Hewitt, L., Crosling, G,. \& Devos, A. (2012). Social inclusion and the student experience: What are the implications for academic support? Widening Participation and Lifelong Learning, 14(2), 11-28. https://doi.org/10.5456/wpll.14.2.11

Bjarnason, T. \& Thorlindsson, T. (2006). Should I stay or should I go? Migration expectations among youth in Icelandic fishing and farming communities. Journal of Rural Studies, 22, 290-300. https://doi:10.1016/j.jrurstud.2005.09.004

Blackwell, D. L., \& McLaughlin, D. K. (1999). Do rural youth attain their educational goals? Rural Development Perspectives, 13(3), 37-44.

Boudon, Raymond, (1974) Education, opportunity, and social inequality: Changing prospects in western society, John-Wiley \& Sons, New York.

Bourdieu, P. (1984). Distinction: A social critique of the judgement of taste (R. Nice, Trans.). Harvard University Press. (Original work published 1977).

Bourdieu, P. (1993). Sociology in question (R. Nice, Trans.). Sage Publications. (Original work published 1984).

Bourdieu P. (1999). The weight of the world: Social suffering in contemporary society. Cambridge: Polity Press.

Bradbury, B. L. \& Mather, P. C. (2009). The integration of first-year, first-generation college students from Ohio Appalachia. NASPA Journal, 46(2), 25-281.

Bradley, H. (2017). 'Should I stay or should I go?': Dilemmas and decisions among UK undergraduates. European Educational Research Journal, 16(1), 30-44. https://doi.org/10.1177/1474904116669363 
Briggs, M. S. (2010). On the outside looking in: A qualitative study of Southern Appalachian first generation students' perceptions of higher education. Retrieved from https://dc.etsu.edu/etd/1758

Brooks, R. G., Walsh M., Mardon, R. E., Lewis, M. \& Clawson, A. (2002). The roles of nature and nurture in the recruitment and retention of primary care physicians in rural areas: A review of the literature. Academic Medicine, 77(8), 790-798.

Brosnan, C., Southgate, E., Outram, S., Lempp, H., Wright, S, Saxby, T., Harris, G., Bennett, A., Kelly, B. (2016). Experiences of medical students who are first in family to attend university. Medical Education. 50(8): 842-851.

Brown, C., Abdelrahman, T., Patel, N., Lorwerth, A., Pollitt, J., Holt, M. \& Lewis, W. G. (2018). Rural rotations at core: Rarefied exposure or real experience? Journal of Surgical Education, 75, 43-48. https://doi.org/10.1016/j.jsurg.2017.06.019

Brown, R., Copeland, W. E., Costello, E. J., Erkanli, A. \& Worthman, C. (2009). Family and community influences on educational outcomes among Appalachian youth. Journal of Community Psychology, 37(7), 795-808. https://doi.org/10.1002/jcop.20331

Bryan, E. \& Simmons, L. A. (2009). Family involvement: Impact on post-secondary educational success for first-generation Appalachian college students. Journal of College Student Development, 50(4), 391-406. https://doi.org/10.1353/csd.0.0081

Bush, J. T. (2015). The Experience of Transition as told by First-Year, First-Generation Appalachian College Students [Doctoral dissertation, West Virginia University]. ProQuest Dissertations \& Theses Global. 
Byrd, K. L. \& MacDonald, G. (2005). Defining college readiness from the inside out: Firstgeneration college student perspectives. Community College Review, 33(1), 22-37. https://doi.org/10.1177/009155210503300102

Byun, S., Irvin, M. J., \& Meece, J. L. (2015). Rural-nonrural differences in college attendance patterns. Peabody Journal of Education, 90(2), 263-279. https://doi.org/10.1080/0161956X.2015.1022384

Byun, S., Meece, J. L., \& Irvin, M. J. (2012). Rural-nonrural disparities in postsecondary educational attainment revisited. American Educational Research Journal, 49(3), 412437. https://doi.org/10.3102/0002831211416344

Byun, S., Meece, J. L., Irvin, M. J. \& Hutchins, B. C. (2012). The role of social capital in educational aspirations of rural youth. Rural Sociology, 77(3), 355-379. https://doi.org/10.1111/j.1549-0831.2012.00086.x

Carr, P. \& Kefalas, M. J. Hollowing Out the Middle: The Rural Brain Drain and What It Means for America, Beacon Press, 2009. ProQuest Ebook Central, http://ebookcentral.proquest.com/lib/wvu/detail.action?docID=3118062

Carrico, C. \& Matusovich, H. M. (2016). A qualitative examination of rural central Appalachian high school student knowledge of the college processes needed to meet career goals. Journal of Women and Minorities in Science and Engineering, 22(3), 259-280.

Cataldi, E. F., Bennett, C. T., Chen, X. (2018). First-generation students: College access, persistence, and postbachelor's outcomes. National Center for Education Statistics: Institute of Education Sciences. Retrieved from https://files.eric.ed.gov/fulltext/ED580935.pdf 
Caygill, R., Peardon, M., Waite, C. \& Wright, J. (2017). Comparing a longitudinal integrated clerkship with traditional hospital-based rotations in a rural setting. Medical Teacher, 39(5), 520-526. https://doi.org/10.1080/0142159X.2017.1297893

Center for Disease Control and Prevention [CDCP], (2014 March 10). NCHHSTP Social Determinants of Health: Definitions. Retrieved from https://www.cdc.gov/nchhstp/socialdeterminants/definitions.html

Chen, F., Fordyce, M., Andes, S. \& Hart, L. G. (2010). Which medical schools produce rural physicians? A 15-year update. Academic Medicine, 85(4), 594-598.

Chenoweth, E. \& Galliher, R. V. (2004). Factors influencing college aspirations of rural West Virginia high school students. Journal of Research in Rural Education, 19(2), 114. http://jrre.psu.edu/articles/19-2.pdf

Choy, S. (2001). Students whose parents did not go to college: Postsecondary access, persistence, and attainment, Washington, DC: National Center for Education Statistics.

Christie, H., Tett, L., Cree, V. E., Hounsell, J., \& McCune, V. (2008). 'A real rollercoaster of confidence and emotions': Learning to be a university student. Studies in Higher Education, 33(5), 567-581. https://doi.org/10.1080/03075070802373040

Coleman, J.S., 1988. Social capital in the creation of human capital. American Journal of Sociology 94, 95-120.

Coleman, J.S., 1995. Achievement-Oriented School Design. In: Hallinan, M. (Ed.), Restructuring Schools: Promising Practices and Policy. Plenum, New York, pp. 11-30.

Collier, P. \& Morgan, D. (2008). "Is that paper really due today?": Differences in first-generation and traditional college students' understandings of faculty expectations. Higher Education, 55(4), 425-446. https://doi.org/10.1007/s10734-007-9065-5 
Conway-Hicks, S. \& de Groot, J. M. (2019). Living in two worlds: Becoming and being a doctor among those who identify with "not form an advantaged background". Current Problems in Pediatric and Adolescent Health Care, 49(4), 92-

101. https://doi.org/10.1016/j.cppeds.2019.03.006

Corbett, M. (2005). Rural education and out-migration: The case of a coastal community. Canadian Society for the Study of Education, 28(1/8), 5272. https://www.jstor.org/stable/1602153

Covarrubias, R., \& Fryberg, S. (2015). Movin' on up (to college): First-generation college students' experiences with family achievement guilt. Cultural Diversity and Ethnic Minority Psychology, 21(3), 420-429. https://doi.org/10.1037/a0037844

Cox, G. R., Tucker, C. J., Sharp, E. H., Van Gundy, K. T., \& Rebellon, C. J. (2014). Practical considerations: Community context in a declining rural economy and emerging adults' educational and occupational aspirations. Emerging Adulthood, 2(3), 173-183. https://doi.org/10.1177/2167696813515850

Crump, A. M., Jeter, K. Mullins, S., Shadoan, A., Ziegler, C. \& Crump, W. J. (2019). Rural medicine realities: The impact of immersion on 0urban-based medical students. The Journal of Rural Health, 35, 42-48. https://doi.org/10.1111/jrh.12244

Crump, W. J. \& Fricker, R. S. (2015). A medical school prematriculation program for rural students: Staying connected with place, cultivating a special connection with people. Teaching and Learning in Medicine, 27(4), 422-430.

https://doi.org/10.1080/10401334.2015.1077709 
Crump, W. J., Fricker, R. S., Flick, K. F., Gerwe-Wickham, K., Greenwell, K., \& Willen, K. L. (2014). A rural pathways program for high school students: Reinforcing a sense of place. Family Medicine, 46(9), 713-717.

Crump, W. J., Fricker, R. S., Ziegler, C. H., Wiegman, D. L. (2016). Increasing the rural physician workforce: A potential role for small rural medical school campuses. The Journal of Rural Health, 32, 254-259. https://doi.org/10.1111/jrh.12156

Curran, V. \& Rourke, J. (2004). The role of medical education in the recruitment and retention of rural physicians. Medical Teacher, 26(3), 265-272. https://doi.org/10.1080/0142159042000192055

Darling, R. A. (1999). The experience of rural, southern Appalachian, first-generation college students at a university: A narrative study [Doctoral dissertation, University of Tennessee, Knoxville]. ProQuest Dissertations \& Theses Global.

Delong, A. W. (2003). Parents of first-generation college students: Their perceptions on the importance of college (3119228) [Doctoral dissertation, The Ohio State University]. ProQuest Dissertations Publishing.

Demetriou, C., Neeve, J., \& Eaker-Rich, D. \& Powell, C. (2017). The activities, roles, and relationships of successful first-generation college students. Journal of College Student Development, 58(1), 19-36. https://doi.org/10.1353/csd.2017.0001

Demi, M., Coleman-Jensen, A., \& Snyder, A. (2010). The rural context and post-secondary school enrollment: An ecological systems approach. Journal of Research in Rural Education, 25(7). Retrieved from http://jrre.psu.edu/articles/25-7.pdf.

Dennis, J. M., Phinney, J. S., \& Chuateco, L. I. (2005). The role of motivation, parental support, and peer support in the academic success of ethnic minority first-generation college 
students. Journal of College Student Development, 46(3), 223-236.

https://doi.org/10.1353/csd.2005.0023

Diversity Policy for the Recruitment of Students and Staff, Faculty (2019). West Virginia

University School of Medicine. Retrieved from

https://medicine.hsc.wvu.edu/media/367061/diversity-policy-for-recruitment-of-studentsfaculty-and-staff.pdf

Donaldson, G. A. (1986). Do you need to leave home to grow up? The rural adolescent's dilemma. Research in Rural Education, 3(3), 121-125.

Drozd, B. L. (2008). Comparisons of first-generation and Non-first-generation College Students on Academic and Psychosocial Measures. [Doctoral dissertation, University of Northern Colorado] ProQuest Dissertations and Theses.

Dumais, S. \& Ward, A. (2010). Cultural capital and first-generation college success. Poetics, 38(3), 245-265. https://doi.org/10.1016/j.poetic.2009.11.011

Dunbabin, J.S., McEwin, K., Cameron, I. (2006). Postgraduate medical placements in rural areas: their impact on the rural medical workforce. Rural and Remote Health, 6(2), 1-10.

Dunstan, S. B. (2013). The Influence of Speaking a Dialect of Appalachian English on the College Experience [Doctoral dissertation, North Carolina State University]. ProQuest Dissertations \& Theses Global.

Dunstan, S. B. \& Jaeger, A. J. (2015). Dialect and influences on the academic experiences of college students. The Journal of Higher Education, 86(5), 777-803. https://doi.org/10.1353/jhe.2015.0026 
Dyk, P. H. \& Wilson, S. M. (1999). Family-based social capital considerations as predictors of attainments among Appalachian youth. Sociological inquiry, 69(3), 477-503. https://doi.org/10.1111/j.1475-682X.1999.tb00882.x

Eakin, E. (2001, January 6). The intellectual class struggle. The New York Times. Retrieved from https://www.nytimes.com/2001/01/06/arts/the-intellectual-class-struggle.html

Egan, M. (1993). Appalachian women: The path from the "hollow" to higher education. Affilia: Journal of Women and Social Work, 8(3), 265-276.

Elam, C. (2002). Culture, poverty and education in Appalachian Kentucky. Education and Culture, 18(1), 10-13.

Elder, G., King, V., \& Conger, R. (1996). Attachment to place and migration prospects: A developmental perspective. Journal of Research on Adolescence, 6(4), 397-425.

Eley, D. \& Baker, P. (2006). Does recruitment lead to retention? Rural clinical school training experiences and subsequent intern choices. Rural and Remote Health, 6(511), 1-11.

Engle, J., \& Tinto, V. (2008). Moving beyond access: College success for low-income, firstgeneration students. The Pell Institute. Retrieved from https://files.eric.ed.gov/fulltext/ED504448.pdf

Federal Student Aid: An Office of the US Department of Education. (n.d.a.). Teacher Cancellation Low Income. Retrieved from https://studentaid.gov/tcli/

Federal Student Aid: An Office of the US Department of Education. (n.d.b.). What factors do states use to determine if a school qualifies as a low-income school and can be listed in the Teacher Cancellation Low Income (TCLI) Directory? Retrieved from https://studentaid.gov/help-center/answers/article/how-to-determine-if-school-qualifiesas-low-income-school-tcli 
Feinstein, L., Duckworth, K. and Sabates, R. (2004a). A model of the inter-generational transmission of educational success: Wider benefits of learning research, report 10. London: Centre for Research on the Wider Benefits of Learning, Institute of Education.

Feinstein, L., Duckworth, K. and Sabates, R. (2004b). A model of the inter-generational effects of parental education: Research brief RCB01 - 04. Nottingham: Department for Education and Skills.

Florence, J. A., Goodrow, B., Wachs, J., Grover, S. \& Olive, K. E. (2007). Rural health professions education at East Tennessee State University: Survey of graduates from the first decade of the community partnership program. The Journal of Rural Health, 23(1), 77-83. https://doi.org/10.1111/j.1748-0361.2006.00071.x

Foster, K. \& Main, H. (2016). Finding a Place in the World: Understanding Youth Outmigration from Shrinking Rural Communities. Report from Social Sciences and Humanities Research Council. Retrieved from https://dalspace.library.dal.ca/bitstream/handle/10222/73932/Finding $\% 20 \mathrm{a} \% 20 \mathrm{Place} \% 20$ v1.pdf? sequence $=1 \&$ isAllowed $=\mathrm{y}$

Franklin, S. L., \& Slate, J. R. (2012). First-generation enrollment and attainment beyond the baccalaureate. Journal of Education Research, 6(2), 175-186.

Friesen, L. \& Purc-Stephenson, R. J. (2016). Should I stay or should I go? Perceived barriers to pursuing a university education for persons in rural areas. Canadian Journal of Higher Education, 46(1), 138-155.

Gallagher, J. M. (2019). First-Generation Central Appalachian Students' Process of Becoming a College Student: A Grounded Theory [Doctoral dissertation, Capella University]. ProQuest Dissertations \& Theses Global. 
Gardner, S. K. \& Holley, K. A. (2011). “Those invisible barriers are real”: The progression of first-generation students through doctoral education. Equity \& Excellence in Education, 44(1), 77-92. https://doi.org/10.1080/10665684.2011.529791

Giardina, D. (1987). Storming Heaven: A Novel. Ivy Books.

Gibbons, M. \& Borders, L. (2010). Prospective first-generation college students: A socialcognitive perspective. Career Development Quarterly, 58, 194-208.

Gibbons, M. M., Taylor, A. L., Brown, E., Daniels, S., Hardin, E. E. \& Manring, S. (2020). Assessing postsecondary barriers to rural Appalachian high school students. Journal of Career Assessment, 28(1), 165-181. https://doi.org/10.1177/1069072719845329

Gibbons, M., Rinehart, A., \& Hardin, E. (2019). How First-Generation College Students Adjust to College. Journal of College Student Retention: Research, Theory, \& Practice, 20(4), 488-510. https://doi.org/10.1177/1521025116682035

Gibbs, R. (1995). Going away to college and wider urban job opportunities take highly educated youth away from rural areas. Rural Development Perspectives, 10(3), 35-44.

Glass, D. V. (1998). "Introduction" in Social mobility in britain in the international library of sociology race, class, and social structure in 21 volumes. Routledge: Oxford. Original work published 1954.

Gofen, A. (2009). Family capital: How first-generation higher education students break the intergenerational cycle. Family Relations, 58(1), 104-120. https://doi.org/10.1111/j.1741$\underline{3729.2008 .00538 . x}$

Gore, J. S., Wilburn, K. R., Treadway, J. \& Plaut, V. (2011). Regional collectivism in Appalachia and academic attitudes. Cross-Cultural Research, 45(4), 376-398. https://doi.org/10.1177/1069397111403396 
Grayson, J. P. (2011). Cultural capital and academic achievement of first generation domestic and international students in Canadian university. British Educational Research Journal, 37(4), 605-630. http://www.jstor.com/stable/23076342

Greenhalgh, T., Seyan, K. \& Boynton, P. (2004). "Not a university type”: focus group study of social class, ethnic, and sex differences in school pupils' perceptions about medical school. British Medical Journal, 328, 1-6.

Griffin, D., Hutchins, B. C., \& Meece, J. L. (2011). Where do rural high school students go to find information about their futures? The American Counseling Association, 89, 172-181.

Gross, D. A., Bates, P., Knox, T. L., \& Gayheart, M. W. (2012). Mining for future health care providers in Appalachian Kentucky. Journal of Health Care for the Poor and Underserved, 23(2), 694-704. https://doi.org/10.1353/hpu.2012.0068

Groves, O. \& O’Shea, S. (2019). Title of article. International Journal of Educational Research, 98, 48-54. https://doi.org/10.1016/j.ijer.2019.08.014

Haggerty, T., Hanks, H., Xiang, J., Unger, K. \& Dino, G. (2019). A comparison of rural and academic training environments for third-year medical students on a family medicine rotation. Family Medicine, 51(5), 430-433. https://doi.org/10.22454/FamMed.2019.893411

Hand, C. \& Payne, E. M. (2008). First-generation college students: A study of Appalachian student success. Journal of Developmental Education, 32(1), 4-15.

Harris, P.A., Taylor, R., Minor, B.L., Elliott, V., Fernandez, M., O’Neal, L., McLeod, L., Delacqua, G., Delacqua, F., Kirby, J., Duda, S.N. (2019). REDCap Consortium, The REDCap consortium: Building an international community of software partners, $J$. Biomed Inform. doi: 10.1016/j.jbi.2019.103208 
Harris, P.A., Taylor, R., Thielke, R., Payne, J., Gonzalez, N., \& Conde, J.G. (2009). Research electronic data capture (REDCap) - A metadata-driven methodology and workflow process for providing translational research informatics support, J Biomed Inform, 42(2): 377-381.

Hektner, J. M. (1995). When moving up implies moving out: Rural adolescent conflict in the transition to adulthood. Journal of Research in Rural Education, 11(1), 3-14.

Helton, L. R. \& Keller, S. M. (2010). Appalachian women: A study of resiliency assets and cultural values. Journal of Social Services Research, 36(2), 151-161. https://doi.org/10.10800/01488370903578124

Hendrickson, K. A. (2012). Student resistance to schooling: Disconnections with education in rural Appalachia. The High School Journal, 95(4), 37-

49. https://www.jstor.org/stable/23275416

Henry, J., Edward, B. \& Crotty, B. (2009). Why do medical graduates choose rural careers? Rural and Remote Health, 9(1083). www.rrh.org.au/journal/article/1083

Herd, M. S., Bulsara, M. K., Jones, M. P., Mak, D. B. (2017). Preferred practice location at medical school commencement strongly determines graduates' rural preference and work location. Australian Journal of Rural Health, 25, 15-21. https://doi.org/10.1111/ajr.12301

Hicks, T. (2003). First-Generation and Non-First-Generation Pre-College Students' Expectations and Perceptions About Attending College. Journal of College Orientation, Transition, and Retention, 11(1), 5-17.

Hicks, T. (2006). Assessing the effects of parental involvement on first-generation and secondgeneration college students (working paper). Fayetteville State University: Faculty 
Working Papers from the School of Education, 12-17.

https://digitalcommons.uncfsu.edu/soe faculty_wp/5

Hinz, S. E. (2016). Upwardly mobile: Attitudes toward the class transition among firstgeneration college students. Journal of College Student Development, 57(3), 285-299. https://doi.org/10.1353/csd.2016.0033

Hlinka, K. R. (2017). Tailoring retention theories to meet the needs of rural Appalachian community college students. Community College Review, 45(2), 144-164. https://doi.org/10.1177/0091552116686403

Holbrook, S. (2016). Developmental Education: Resilience and Academic Success in Rural Appalachian Students” [Doctoral dissertation, Eastern Kentucky University]. ProQuest Dissertations \& Theses Global.

Howley, C. Harmon, H., \& Leopold, G. (1997). Rural scholars or bright rednecks? Aspirations for a sense of place among rural youth in Appalachia. Report for Appalachia Educational $L a b$.

Hsiao, K. P. (1992). First-generation college students. Eric Digest. https://eric.ed.gov/?id=ED351079

Hunley, F. M. (2015). Understanding Factors Contributing to the Persistence of FirstGeneration College Students from Appalachian Distressed Counties: A Phenomenological Study [Doctoral dissertation, Liberty University]. ProQuest Dissertations \& Theses Global.

Hurst, A. L. (2007). Telling tales of oppression and dysfunction: Narratives of class-identity reformation. Qualitative Sociology Review, 3(2), 82-104. 
Hurst, A. L. (2010). The burden of academic success: Loyalists, renegades, and double agents. Lanham, MD: Lexington.

Hutchins, B. C., Meece, J. L., Byun, S. \& Farmer, T. W. (2012). Planning for the future: An investigation of work-bound rural youth. Rural Education, 33(2), 7-19.

Irvin, M., Byun, S. Y., Smiley, W. S. \& Hutchins, B. C. (2017). Relation of opportunity to learn advanced math to the educational attainment of rural youth. American Journal of Education, 123, 475-510.

Isaac, V., Watts, Forster, L., McLachlan, C. S. (2014). The influence of rural clinical school experiences on medical students' levels of interest in rural career. Resource for Health, $12(48), 1-6$.

Jenkins, A., Miyazaki, Y. \& Janosik, S. (2009). Predictors that distinguish first-generation college students from non-first generation college students. Journal of Multicultural, Gender and Minority Studies, 3(1), pages 1-9.

Jenkins, S. A., Belanger, A., Connally, M. L., Boals, A., \& Duror, K. M. (2013). First-generation undergraduate students' social support, depression, and life satisfaction. Journal of College Counseling, 16(2), 129-142. https://doi.org/10.1002/j.2161-1882.2013.00032.x

Johnson, M. K., Elder, G. H., \& Stern, M. (2005). Attachments to family and community and the young adult transition of rural youth. Journal of Research on Adolescence, 15(1), 99-125.

Jolly, P. (2008). Diversity of U.S. medical students by parental income. AAMC Analysis in Brief, 8(1), 1-2. https://www.aamc.org/media/5776/download

Jones, M., Humphreys, J., Prideaux, D. (2009). Predicting medical students' intentions to take up rural practice after graduation. Medical Education, 43, 1001-1009. https://doi.org/10.1111/j.1365-2923.2009.03506.x 
Jones, P. S. (2002). Dialect as a deterrent to cultural stripping: Why Appalachian migrants continue to talk that talk. In P. J. Obermiller \& M. E. Maloney (Eds.), Appalachia: Social context past and present (4 ${ }^{\text {th }}$ ed., pp. 151-154). Dubuque, IA: Kendall/Hunt.

Jury, M., Smeding, A., Court, M, \& Darnon, C. (2015). When first-generation students succeed at university: On the link between social class, academic performance, and performanceavoidance goals. Contemporary Educational Psychology, 41, 25-36. doi:10.1016/j.cedpsych.2014.11.001

Jury, M., Smeding, A., Stephens, N. M., Nelson, J. E., Aelenei, C. \& Darnon, C. (2017). The Experience of Low-SES Students in Higher Education: Psychological Barriers to Success and Interventions to Reduce Social-Class Inequality. Journal of Social Issues, 73(1), 2341. https://doi.org/10.1111/josi.12202

Kim, Y. K. \& Sax, L. J. (2009). Student-faculty interaction in research universities: Differences by student gender, race, social class, and first-generation status. Research in Higher Education, 50, 437-459. https://doi.org/10.1007/s11162-009-9127-x

King, S, Luzeckyj, A. \& McCann, B. (2019). The experience of being first in family at university: Pioneers in higher education. Springer: Singapore. Retrieved from https://ebookcentral.proquest.com/lib/wvu/reader.action?docID $=5967228$

Kondalsamy-Chennakesavan, Eley, D. S., Ranmuthugala, G., Chater, A. B., Toombs, M. R., Darshan, D. \& Nicholson, G. (2015). Determinants of rural practice: Positive interaction between rural background and rural undergraduate training. Medical Journal of Australia, 202(1), 41-46. https://doi.org/10.5694/mja14.00236

Kuh, G., Kinzie, J., Schuh, J. H., Whitt, E. J., \& Associates. Student success in college: Creating conditions that matter. Jossey-Bass. 
Lang, F., Ferguson, K. P., Bennard, B., Zahorik, P., Sliger, C. (2005). The Appalachian preceptorship : Over two decades of an integrated clinical-classroom experience of rural medicine and Appalachian culture. Academic Medicine, 80(8), 717-723. https://doi.org/10.1097/00001888-200508000-00002

Lehmann, W. (2009). Becoming middle class: How working-class university students draw and transgress moral class boundaries. Sociology, 43(4), 631-647. https://doi.org/10.1177/0038038509105412

Lichter, D., \& Brown, D. (2011). Rural American in an urban society: Changing spatial and social boundaries. Annies Review of Sociology, 37, 565-592. https://doi.org/10.1146/annurev-soc-081309-150208

Lohfink, M. M. \& Paulsen, M. B. (2005). Comparing the Determinants of Persistence for FirstGeneration and Continuing-Generation Students. Journal of College Student Development, 46(4), 409-428. https://doi.org/10.1353/csd.2005.0040

London, H. B. (1989). Breaking away: A study of first-generation college students and their families. American Journal of Education, 97(2), 144170. https://www.jstor.org/stable/1084908

Luceckyj, A., McCann, B., Graham, C., King, S., \& McCann, J. (2017). Being First in Family: motivations and metaphors. Higher Education Research and Development, 36(6), 12371250. https://doi.org/10.1080/07294360.2017.1300138

Luke, H. (2003). Medical education and sociology of medical habitus: it's not about the stethoscope! Kluwer Academic Publishers: New York. 
Manly, C. A., Wells, R. S., \& Kommers, S. (2020). Who are rural students? How definition of rurality affect research on college completion. Research in Higher Education, 61, 764779.

Marshall, J. L, Thomas, L., Lane, N. M., Holmes, G. M., Arcury, T. A, Randolph, R., Silberman, P., Holidng, P., Holding, W., Villamil, L., Thomas, S., Lane, S., Latus, J., Rodger, J., \& Ivey, K. (2017 August 23). Health disparities in Appalachia. The first report in a series exploring health issues in Appalachia. Retrieved from https:/www.arc.gov/wpcontent/uploads/2020/06/Health_Disparities_in_Appalachia_August_2017.pdf

Martinez, J. A., Sher, K. J., Krull, J. L., \& Wood, P. K. (2009). Blue-collar scholars?: Mediators and moderators of university attrition in first-generation college students. Journal of College Student Development, 50(1), 87-103. https://doi.org/10.1353/csd.0.0053

Mason, H. R. C., Winseman, J., Marcellon, R., Huamantla, M., Ruiz, C. Ayala, E. E. (2018). First-generation medical student wellness in the United States: A cross-sectional snapshot. Journal of Best Practices in Health Professions Diversity: Education, Research \& Policy, 11(2), 96-106.

Mathers, J. \& Parry, J. (2009). Why are there so few working-class applicants to medical schools? Learning form the success stories. Medical Education, 43, 219-228. https://doi.org/10.1111/j.1365-2923.2008.03274.x

Maton, K. (2012). Habitus. In M. J. Grenfell (Ed.), Pierre Bourdieu: Key Concepts $\left(2^{\text {nd }}\right.$ Ed.) (pp. 48-64). Acumen Publishing Limited.

Matsumoto, M., Inoue, K. \& Kajii, E. (2008). Characteristics of medical students with rural origin: Implications for selective admission policies. Health Policy, 87 (2), 194-202. https://doi.org10.1016/j.healthpol.2007.12.006 
Maxfield, H., Kennedy M., Delzell, J. E. \& Paolo, A. M. (2014). Performance of third-year medical students on a rural family medicine clerkship. Family Medicine, 46(7), 536-538.

McCarron, G. P. \& Inkelas, K. K. (2006). The gap between educational aspirations and attainment for first-generation college students and the role of parental involvement. Journal of College Student Development, 47(5), 535-549. https://doi.org/10.1353/csd.2006.0059

McDonald, C. L. (2004). West Virginia counties.png. Retrieved from https://commons.wikimedia.org/wiki/File:West_Virginia_Counties.png

McGrail, M. R., Wingrove, P. M., Petterson, S. M. \& Bazemore, A. W. (2017). Mobility of US rural primary care physicians during 2000-2014. Annals of Family Medicine, 15(4), 322328. https://doi.org/10.1370/afm.2096

McHarg J., Mattick, K. \& Knight, L. V. (2007). Why people apply to medical school: Implications for widening participation activities. Medical Education, 41(8), 815-821. https://doi.org/10.1111/j.1365-2923.2007.02798.x

McInnis, C. \& James, R. (1995). First year on campus: Diversity in the initial experiences in Australian undergraduates. Centre for the Study of Higher Education: University of Melbourne. https://www.researchgate.net/publication/243784496

Means, D., \& Pyne, K. (2017). Finding my way: Perceptions of institutional support and belonging in low-income, first-generation, first-year college students. Journal of College Student Development, 58(6), 907-924. https://doi.org/10.1353/csd.2017.0071

Meece, J. L., Askew, K. J. S., Agger, C. A., Hutchins, B. C., \& Byun, S. (2014). Familial and economic influences on the gender-related educational and occupation aspirations of rural 
adolescents. Journal of Educational Development Psychology, 4(1), 238-257.

https://doi.org/10.5539/jedp.v4n1p238

Meece, J. L., Hutchins, B. C., Byun, S., Farmer, T. W., Irvin, M. \& Weiss, M. (2013). Preparing for adulthood: A recent examination of the alignment of rural youth's future educational and vocational aspirations. Journal of Educational and Developmental Psychology, 3(2), 175-192. https://doi.org/10.5539/jedp.v3n2p175

Meehan, M. L., Cowley, K. S., Chadwick, K. L., \& Whittaker, D. (2001). Academic aspirations of Appalachian seventh graders and their parents. Paper presented at the Annual Meeting of the American Educational Research Association, Seattle, Washington.

Mehta, S. S., Newbold, J. J. \& O’Rourke, M. A. (2011). Why do first-generation students fail? College Student Journal, 45(1).

Merriam, S., \& Tisdell, S. (2016). Qualitative research: A Guide to design and implementation, 4th Ed. Jossey-Bass.

Moody, J. (2020 March 17). 10 Med Schools with the Lowest Acceptance Rates. US News \& World Report. Retrieved from https://www.usnews.com/education/best-graduateschools/the-short-list-grad-school/articles/medical-schools-with-the-lowest-acceptancerates

Moore, R. (2012). Capital. In M. J. Grenfell (Ed.), Pierre Bourdieu: Key Concepts (2 ${ }^{\text {nd }}$ Ed.) (pp. 98-112). Acumen Publishing Limited.

Moores, D. G., Woodhead-Lyons, S. C. \& Wilson, D. R. (1998). Preparing for rural practice: Enhanced experience for medical students and residents. Canadian Family Physician, 44, 1045-1050. 
Morse, C. E. \& Mudgett, J. (2018). Happy to be home: Place-based attachments, family ties, and mobility among rural stayers. The Professional Geographer, 70(2), 261-269. https://doi.org/10.1080/00330124.2017.1365309

Morton, T. R., Ramirez, N. A., Meece, J. L., Demetriou, C. \& Panter, A. T. (2018). Perceived barriers, anxieties, and fears in prospective college students from rural high schools. The High School Journal, 101(3), 155-176. https://www.jstor.org/stable/10.2307/90024241

National Resident Matching Program. (2021). About. Retrieved from https://www.nrmp.org/about-nrmp/

Nicholson, S. (2013). Exploring the academic experience of medical students from a nontraditional socio-economic background: A study of their models of learning and professionalisation with an undergraduate medical curriculum. [Doctoral Dissertation, Institute of Education: University of London]. UCL Discovery. Retrieved from https://discovery.ucl.ac.uk/id/eprint/10018063/2/PhD\%20manuscript $\% 20$ Sandra $\% 20 \mathrm{Nich}$ olson\%20pdf.pdf

Nicholson, S. \& Cleland, J. A. (2017). "It's making contacts": Notions of social capital and implications for widening access to medical education. Advances in Health Sciences Education, 22, 477-490. https://doi.org/10.1007/s10459-016-9735-0

Nunez, A. \& Cuccaro-Alamin, S. (1998). First-generation students: Undergraduates whose parents never enrolled in Post-secondary education. Washington, DC: National Center for Education Statistics.

O'Shea, S. E. (2007). Well I got here ... but what happens next? Exploring the early narratives of first year female students who are the first in the family to attend university. Journal of Australian and New Zealand Student Services Association, 29, p. 36-51 
O'Shea, S. (2015a). Arriving, surviving, and succeeding: First-in-family women and their experiences of transitioning into the first year of university. Journal of College Student Development, 56(5), 499-517. https://doi.org/10.1353/csd.2015.0053

O'Shea, S. (2015b). Filling up silences - first in family students, capital and university talk in the home. International Journal of Lifelong Education, 34:2, 139-155.

O'Shea, S. (2016). Avoiding the manufacture of 'sameness': first-in-family students, cultural capital and the higher education environment. Higher Education, 72(1), 59-78. https://doi.org/10.1007/s10734-015-9938-y

O'Shea, S. \& Delahunty, J. (2019). “That working-class ethic ... where there's a will there's a way": A strengths-based approach to developing employable scholars. In Diver, A. (ed) Employable scholars in HE: Challenges and choices in times of austerity. Springer, UK.

O’Shea, S., May, J., Stone, C., \& Delahunty, J. (2017). First-in-family, students, university experience \& family life: Motivations, Transitions and Participation. Palgrave Macmillan.

Orbe, M. P. (2014). Negotiating multiple identities within multiple frames: An analysis of firstgeneration college students. Communication Education, 53(2), pages 131149. https://doi.org/10.1080/03634520410001682401

Owen, J. A., Conaway, M. R., Bailey, B. A. Hayden, G. F. (2007). Predicting rural practice using different definitions to classify medical school applicants as having a rural upbringing. Journal of Rural Health, 23(2), 133-140. https://doi.org/10.1111/j.1748$\underline{0361.2007 .00080 . x}$ 
Padgett, R., Johnson, M. \& Pascarella, E. (2012). First-generation undergraduate students and the impacts of the first-year of college: Additional Evidence. Journal of College Student Development, 53(2), 243-266. https://doi.org/10.1353/csd.2012.0032

Paladine, H. L., Hustedde, C., Wendling, A., Sola, O., Prasad, R., Bjorkman, S. \& Phillips, J. (2020). The role of rural communities in the recruitment and retention of women physicians. Women \& Health. 60(1), 113-122. https://doi.org/10.1080/03630242.2019.1607801

Pascarella, E. T., Pierson, C. T., Wolniak, G. C., \& Terenzini, P. T. (2004). First-generation college students: Additional evidence on college experiences and outcomes. The Journal of Higher Education, 75(3), 249-284. DOI: https://doi.org/10.1353/jhe.2004.0016

Patfield, S., Gore, J. \& Fray, L. (2020). Reframing first-generation entry: how the familial habitus shape aspirations for higher education among prospective first-generation students. Higher Education Research and Development, 1-14. https://doi.org/10.1080/07294360.2020.1773766

Patton, M. Q. (2015). Qualitative research and evaluation methods (4 ${ }^{\text {th }}$ ed.). Thousand Oaks, CA: Sage.

Petrin, R. A., Farmer, T. W., Meece, J. L., \& Byun, S. (2011). Interpersonal competence configurations, attachment to community, and residential aspirations of rural adolescents. Journal of Youth and Adolescence, 40(9), 1091-1105. https://doi.org/10.1007/s10964-011-9690-2

Petrin, R. A., Schafft, K. A., \& Meece, J. L., (2014). Educational sorting and residential aspirations among rural high school students: What are the contributions of schools and 
educators to rural brain drain? American Educational Research Journal, 51(2), 294-326. https://doi.org/10.3102/0002831214527493

Pike, G. \& Kuh, G. (2005). First- and second-generation college students: A comparison of their engagement and intellectual development. Journal of Higher Education, 76(3), 276300. https://doi.org/10.1353/jhe.2005.0021

Playford, D., Ngo, H., Gupta, S., Puddey, I. B. (2017). Opting for rural practice: The influence of medical student origin, intention and immersion experience. Medical Journal of Australia, 207(4), 154-158. https://doi.org/10.5694/mja16.01322

Pollard, K. \& Jacobsen, L.A. (2019). The Appalachian Region: A Data Overview from the 20132017 American Community Survey Chartbook. Population Reference Bureau. Washington, DC. Retrieved from https://www.arc.gov/assets/research_reports/DataOverviewfrom2013to2017ACS.pdf

Powell, S. M. (2008). Perceptions of Appalachian Students about Post-Secondary Education [Doctoral dissertation, Ohio University]. ProQuest Dissertations \& Theses Global.

Puddey, I. B., Mercer, A., Playford, D. E., \& Riley, G. (2015). Medical student selection criteria and socio-demographic factors as predictors of ultimately working rurally after graduation. BMC Medical Education, 15(1), 1-10. https://doi.org/10.1186/s12909-015$0359-5$

Puddey, I., Mercer, A., Playford, D. E., Pougnault, \& Riley, G. J. (2014). Medical student selection criteria as predictors of intended rural practice following graduation. $B M C$ Medical Education, 14(1), 1-12. https://doi.org/10.1186/1472-6920-14-218

Rabinowitz, H. K., Diamond, J. J., Markham, F. W. \& Santana, A. J. (2012). The relationship between matriculating medical students' planned specialties and eventual rural practice 
outcomes. Academic Medicine, 87(8), 1086-1090.

https://doi.org/10.1097/ACM.0b013e31825cfa54

Rabinowitz, H. K., Diamond, J. J., Markham, F. W., \& Paynter, N. (2001). Critical factors for designing programs to increase the supply and retention of rural primary care physicians. Journal of the American Medical Association, 286(9), 1041-1048.

Raitz, K. B., Ulack, R. \& Leinbach, T. R. (2018). Appalachia A Regional Geography: Land, People and Development. Routledge (Originally published 1984).

Ramos-Sanchez, L \& Nichols, L. (2007). Self-efficacy of first-generation and non-firstgeneration college students: The relationship with academic performance and college adjustment. Journal of College Counseling, 10(1), 6-18. https://doi.org/10.1002/j.21611882.2007.tb00002.x

Ranmuthugala, G., Humphreys, J., Solarsh, B., Walters, L., Worley, P., Wakerman, J., Dunbar, J. A. \& Solarsh, G. (2007). Where is the evidence that rural exposure increases uptake of rural medical practice? Australian Journal of Public Health, 15(5), 285-288. https://doi.org/10.1111/j.1440-1584.2007.00915.x

Reay, D. (1998). 'Always knowing' and 'never being sure': Familial and institutional habituses and higher education choice. Journal of Education Policy, 13(4), 519-529. recruitment and retention of rural physicians. Medical Teacher, 26(3):265-72.

Rendón, L. I. (1993). Validating culturally diverse students: Toward a new model of learning and student development. National Center on Postsecondary Teaching, Learning, and Assessment. University Park, PA: Office of Educational Research and Improvement.

Roberts, J. S., \& Rosenwald, G. C. (2001). Ever upward and no turning back: Social mobility and identity formation among first-generation college students. In D. P. McAdams, R. 
Josselson, \& A. Lieblich (Eds.), Turns in the rod: Narrative studies of lives in transition (p. 91-119).

Roscigno, V. \& Crowley, V. (2001). Rurality, institutional disadvantage, and achievement/attainment. Rural Sociology, 66(2), 268-292. https://doi.org/10.1111/j.15490831.2001.tb00067.x

Roscigno, V., Tomaskovic-Devey, D., \& Crowley, M. (2006). Education and the inequalities of place. Social forces, 84(4), 2121-2145. https://doi.org/10.1353/sof.2006.0108

Roseamelia, C., Greenwald, J. L., Bush, T., Pratte, M., Wilcox, J., \& Morley, C. (2014). A qualitative study of medical students in a rural track: Views on eventual rural practice. Family Medicine, 46(4), 259-266.

Rosecrance, P. H., Graham, D., Manring, S., Cook, K. D., Hardin, E. E., \& Gibbons, M. M. (2019). Rural Appalachian high school students' college-going and STEMM perceptions. The Career Development Quarterly, 67, 327-342. https://doi.org/10.1002/cdq.12202

Rosenblatt, R. (2010). The medical schools have a responsibility to train physicians to meet the needs of the public? Academic Medicine. 85(4), 572-574.

Roush, J. R. (2013). Educational Assets, Resources, Barriers and Social Capital in a SemiIsolated Community in Appalachia [Doctoral dissertation, Ohio University] ProQuest Dissertations \& Theses Global.

Royston, PJ, Mathieson, K., Leafman, J. \& Ojan-Sheehan, O. (2012). Medical student characteristics predictive of intent for rural practice. Rural and Remote Health, 12(3), 110. 
RTI International. (2019a). First-generation College Students' Employment. Washington, DC: NASPA. Retrieved from https://firstgen.naspa.org/files/dmfile/FactSheet-04.pdf

RTI International. (2019b). First-generation College Students: Demographic Characteristics and Postsecondary Enrollment. Washington, DC: NASPA. Retrieved from https://firstgen.naspa.org/files/dmfile/FactSheet-01.pdf

RTI International. (2019c). First Year Experience, Persistence, and Attainment of Firstgeneration College Students. Washington, DC: NASPA. Retrieved from https://firstgen.naspa.org/files/dmfile/FactSheet-02.pdf

RTI International. (2019d). Use of Student Services among Freshman First-generation College Students. Washington, DC: NASPA. Retrieved from https://firstgen.naspa.org/files/dmfile/NASPA_FactSheet-03_FIN.pdf

Schademan, A., \& Thompson, M. (2015). Are college faculty and first-generation, low-income students ready for each other? Journal of College Student Retention: Research, Theory and Practice, 1-23. https://doi.org/10.1177/1521025115584748

Schmitt-Wilson, S. (2013). Social class and expectations of rural adolescents: the role of parental expectations. Career Development Quarterly, 61(3), 226-239. https://doi.org/10.1002/j.2161-0045.2013.00051.x

Seay, S. E., Lifton, D. E., Wuensch, K. L., Bradshaw, Lynn, K., \& McDowelle, J. O. (2008). First-generation graduate students and attrition risks. The Journal of Continuing Higher Education, 56(3), 11-25. https://doi.org/10.1080/07377366.2008.10400158

Shannon, C. K. \& Jackson, J. (2015). Validity of medical student questionnaire data in prediction of rural practice choice and its association with service orientation. The Journal of Rural Health, 31(4), 373-381. 
Shannon, C.K., Baker, H., Jackson, J., Roy, A., Heady, H. \& Gunel, E. (2005). Evaluation of a required statewide interdisciplinary rural health education program: Student attitudes career intents and perceived quality. Rural and Remote Health, 5(4), 1-8.

Singh, K. \& Dika, S. (2003). The educational effects of rural adolescents' social networks. Journal of Research in Rural Education, 18(2), 114-128

Smith, C. C. (2012). Investigating a rural immersion experience in medical education utilizing narrative inquiry. Narrative Inquiry in Bioethics, 2(1), 56-64. https://doi.org/10.1353/nib.2012.0005

Snyder, A., McLaughlin, D. \& Coleman-Jensen, A. (2009). The New, Longer Road to Adulthood: Schooling, Work, and Idleness among Rural Youth. A Casey Institute Report. $1-20$.

Snyder, T. D. (2007). The Webster county blues: An exploration of the educational attitudes of a poor Appalachian community. Community Literacy Journal, 2 (1), 91-106.

Somporn, P., Ash, J. \& Walters, L. (2018). Stakeholder views of rural community-based medical education: A narrative review of the international literature. Medical Education, 52(8), 791-802. https://doi.org/10.1111/medu. 13580

Soria, K. M. \& Stebleton, M. (2012). First-generation students' academic engagement and retention. Teaching in Higher Education, 17(6), 673685. http://dx.doi.org/10.1080/13562517.2012.666735

Southgate, E., Brosnan, C., Lempp, H., Kelly, B., Wright, S., Outram, S. \& Bennett, A. (2017). Travels in extreme social mobility: How first-in-family students find their way into and through medical education. Critical Studies in Education, 58(2), 242-260. https://doi.org/10.1080/17508487.2016.1263223 
Southgate, E., Kelly, B., Symonds, I. (2015). Disadvantage and the 'capacity to aspire' to medical school. Medical Education: 49(1), p. 73-83.

Spenny, M. \& Ellsbury, K. (2000). Perceptions of practice among rural family physicians-Is there a gender difference? Journal of the American Board of Family Practice. 13(3), 183187. https://doi.org/10.3122/15572625-13-3-183

Stagg, P., Greenhill, J. \& Worley, P.S. (2009). A new model to understand the career choice and practice location decisions of medical graduates. Rural and Remote Health, 9(4), 1-10. https://doi.org/10.22605/rrh1245

Stebleton, M. J. \& Soria, K. M. (2012). Breaking down barriers: Academic obstacles of firstgeneration students at research universities. Learning Assistance Review, 17(2), 7-19.

Stegers-Jager, K., Themmen, A. P. N., Cohen-Schotanus, J. \& Steyerberg, E. W. (2015). Predicting performance: Relative importance of students background and past performance. Medical Education, 49(9), 933-945. https://doi.org/10.1111/medu.12779

Stephens, N. M., Fryberg, S., Markus, H. R., Johnson, C. S., \& Covarrubias, R. (2012). Unseen disadvantage: How American universities' focus on independence undermines the academic performance of first-generation college students. Journal of Personality and Social Psychology, 102(6), 1178-1197. https://doi.org/10.1037/a0027143

Stephens, N. M., Townsend, S. S. M., Markus, H. R. \& Phillips, L. T. (2012). A cultural mismatch: Independent cultural norms produce greater increases in cortisol and more negative emotions among first-generation college students. Journal of Experimental Social Psychology, 48(6), 1389-1393. https://doi.org/10.1016/j.jesp.2012.07.008

Stieha, V. (2010). Expectations and experiences: The Voice of a First Generation first year college student and the question of student persistence. International Journal of 
Qualitative Studies in Education, 23(2), 237-249.

https://doi.org/10.1080/09518390903362342

Stockdale, A. \& Haartsen, T. (2018). Editorial introduction: Putting rural stayers in the spotlight. Population, Space and Place, 24(4), 1-8. https://doi.org/10.1002/psp.2124

Swecker, H., Fifolt, M., \& Searby, L. (2013). Academic Advising and First-Generation College Students: A Quantitative Study on Student Retention. NACADA Journal, 43(11), pages. https://doi.org/10.12930/nacada-13-192

Tang, M. \& Russ, K. (2007). Understanding and facilitating career development of people of Appalachian culture: An integrated approach. The Career Development Quarterly, 56, $34-46$.

Tate, K. A., Caperton, W., Kaiser, D., Pruitt, N. T., White, H., \& Hall, E. (2015). An exploration of first-generation college students' career development beliefs and experiences. Journal of Career Development, 42(4), 294-310. https://doi.org/10.1177/0894845314565025

Terenzini, P. T., Rendon, L. I., Upcraft, M. L. Millar, S. B., Allison, K. W., Gregg, P. L., et al. (1994). The transition to college: Diverse students, diverse stories. Research in Higher Education, 35(1), 57-73.

Thomas, L. \& Quinn, J. (2006). First Generation Entry into Higher Education, McGraw-Hill Education. Retrieved from http://ebookcentral.proquest.com/lib/wvu/detail.action?docID $=316328$

Thomson, P. (2012). Field. In M. J. Grenfell (Ed.), Pierre Bourdieu: Key Concepts (2 ${ }^{\text {nd }}$ Ed.) (pp. 65-80). Acumen Publishing Limited. 
Tieken, M. C. (2016). College talk and the rural economy: Shaping the educational aspirations of rural, first-generation students. Peabody Journal of Education, 91(2), 203223. https://doi.org/10.1080/0161956X.2016.1151741

Tinto, V. (1993). Leaving college: Rethinking the causes and cures of student attrition (2 ${ }^{\text {nd }}$ Ed.). The University of Chicago Press.

Tönnies, F. (1887/2001). Tönnies: Community and civil society, edited by Jose Harris, Cambridge University Press. Retrieved from http://ebookcentral.proquest.com/lib/wvu/detail.action?docID=201523.

Tower, G. (2005). West Virginia’s lost youth: Appalachian stereotypes and residential preferences. Journal of Geography, 104, 74-84.

Tran, U. S., Berger, N., Arendasy, M. E., Greitemeyer, T., Himmelbauer, M., Hutzler, F., Kraft, H. G., Oettl, K., Papousek, I., Vitouch, O. \& Voracek, M. (2017). Unto the third generation: Evidence for strong familial aggregation of physicians, psychologists, and psychotherapists among first-year medical and psychology students in a nationwide Austrian cohort census. BMC Medical Education, 17(1), 1-15. https://doi.org/10.1186/s12909-017-0921-4

Trickett-Shockey, A. K., Wilson, C. S., Lander, L. R., Barretto, G. A., Szklarz, G. D., VanVoorhis, G. C. \& Minardi, J. J. (2013). A study of rural upbringing and education on the intent of health professional students to work in rural settings. International Journal of Medical Education, 4. https://doi.org/10.5116/ijme.50f1.48cf

Universal Service Administrative Company (USAC). (2021a). Universal Service. Retrieved from https://www.usac.org/about/universal-service/ 
Universal Service Administrative Company (USAC). (2021b). Urban and Rural Status.

Retrieved from https://www.usac.org/e-rate/applicant-process/applying-fordiscounts/urban-or-rural-status/

US Census Bureau. (2019 December 2). 2010 Census Urban and Rural Classification and Urban Area Criteria. Retrieved from https://www.census.gov/programssurveys/geography/guidance/geo-areas/urban-rural/2010-urban-rural.html

Votruba, D. (Year). Interpersonal guilt and college adjustment among first-generation college students. (3339650) [Doctoral dissertation, University of Michigan, Ann Arbor]. ProQuest Dissertations and Theses.

Vuong, M., Brown-Welty, S., \& Tracz, S. (2010). The effects of self-efficacy on academic success of first-generation college sophomore students. Journal of college Student Development, 51(1), 50-64. https://doi.org/10.1353/csd.0.0109

Wainwright, E., \& Watts, M. (2019). Social mobility in the slipstream: first-generation students’ narratives of university participation and family. Educational Review, 1-17. https://doi.org/10.1080/00131911.2019.1566209

Walker, J. H., DeWitt, D.E., Pallant, J.F. \& Cunningham, C.E. (2012). Rural origin plus a rural clinical placement is a significant predictor of medical students' intentions to practice rurally: A multi-university study. Rural and Remote Health, 12(1), 1-9.

Wallace, L. A. \& Diekroger, D. K. (2000). "The ABCs in Appalachia": A descriptive view of perceptions of high education in Appalachian culture. Paper presented at the Annual conference of the Women of Appalachia: their Heritage and Accomplishments $\left(2^{\text {nd }}\right)$ Zanesville, $\mathrm{OH}$. 
Wasserman, W. (2017 April). Appalachian region endures dramatic health challenges compared with nation, new research shows. Appalachian Regional Commission. Retrieved from https://www.arc.gov/news/appalachian-region-endures-dramatic-health-challengescompared-with-nation-new-research-shows/

Webb-Sunderhaus, S. (2016). "Keep the Appalachian, drop the redneck”: Tellable student narratives of Appalachian identity. College English, 79(1), 11-

33. https://www.jstor.org/stable/44075153n

Welch, F. M. (2013). Difference Does Not Mean Deficient: The Cultural and Higher Education Experiences of Appalachian Women [Doctoral dissertation, Indiana State University]. ProQuest Dissertations \& Theses Global.

Westbrook, S. \& Scott, J. (2012). The influence of parents on the persistence of first-generation college students. Focus on College, University, and Schools, 6 (1), 1-9.

Wettersten, K. B., Guilmino, A., Herrick, C. G., Hunter, P. J., Kim, G. Y., Jagow, D., Beecher, T., Faul, K., Baker, A. A., Rudolph, S. E., Ellenbecker, K., \& McCormick (2005). Predicting educational and vocational attitudes among rural high school students. Journal of Counseling Psychology, 52(4), 658-663. https://doi.org/10.1037/0022-0167.52.4.658

Wheat, J. R., Coleman, V. L., Murphy, S., Turberville, C. M. \& Leeper, J. D. (2015). Medical education to improve rural population health: A chain of evidence from Alabama. The Journal of Rural Health, 31(4), 354-364. https://doi.org/10.1111/jrh.12113

White, A. \& Perrone-McGovern, K. (2017). Influence of Generational Status and Financial Stress on Academic and Career Self-Efficacy. Journal of Employment Counseling, 54, 38-46. https://doi.org/10.1002/joec. 12049 
Wildhagen T. (2015). "Not your typical student": The social construction of the "firstgeneration" college student. Qualitative Sociology, 38(3), 285-303.

Wilson, S. \& Gore, J. (2009). Appalachian origin moderates the association between school connectedness and GPA: Two exploratory studies. Journal of Appalachian Studies, 15(1/2), 70-86. http://???.jstor.com/stable/41446819

Woloschuk, W. \& Tarrant, M. (2002). Does a rural educational experience influence students' likelihood of rural practice? Impact of student background and gender. Medical Education, 36(3), 241-247. https://doi.org/10.1046/j.1365-2923.2002.01143.x

Wood, F. M. (2012). Factors Influencing College Decision-Making for First-Generation Appalachian Student [Doctoral dissertation, Marshall University]. ProQuest Dissertations \& Theses Global.

Woosley, S. \& Shepler, D. (2011). Understanding the Early Integration Experiences of FirstGeneration College Students. College Student Journal, 45(4), 700-714.

Wright, C. J. (2012). Becoming to remain: Community college students and post- secondary pursuits in central Appalachia. Journal of Research in Rural Education, 27(6). http://jrre./psu.edu/articles/27-6.pdf

Wright, D., Cunningham, B., \& Stangle, J. (2016, October 5). The Appalachian Region: Identifying and Addressing the Region's Education Needs. US Department of Education. Washington, DC. Retrieved from https://www2.ed.gov/about/bdscomm/list/rac/appalachian-region.pdf

Wyatt, J. E. \& Chater, A. B. (2018). Academic performance of third-year medical students learning in rural settings. Australian Journal of Rural Health, 26(4), 265-272. https://doi.org/10.1111/ajr.12414 
Wyatt, J. E. \& Lyons, C. M. (2018). Rural health club program focused on strengthening medical students' intention toward a rural medicine career. Australian Journal of Rural Health, 26(4), 273-278. https://doi.org/10.1111/ajr.12420

Yin, R. K. (2018). Case study research and applications design and methods, $6^{\text {th }}$ Ed. Sage

Zink, T., Power, D. V., Olson, K., Harris, I. B. \& Brooks, K. D. (2010). Qualitative difference between traditional and rural-longitudinal medical student OSCE performance. Family Medicine, 42(10), 707-711. 


\section{Appendix 1: Questionnaire for Participants}

1) Student name and email (open ended)

2) List the counties and states where you were raised and spent formative years? (open ended)

3) Amount of education completed by parents or legal guardian?

a. Father (Options: Unknown; less than high school diploma; some college or technical education; four-year college degree; master's degree or comparable; doctoral or terminal degree other than medical doctorate; medical doctorate)

b. Mother (Options: Unknown; less than high school diploma; some college or technical education; four-year college degree; master's degree or comparable; doctoral or terminal degree other than medical doctorate; medical doctorate)

c. Other legal guardian, if applicable (Options: Unknown; less than high school diploma; some college or technical education; four-year college degree; master's degree or comparable; doctoral or terminal degree other than medical doctorate; medical doctorate)

4) Student gender (if you prefer not to respond, please leave blank) (Open ended)

5) Where do you plan to practice medicine after residency training? (Open ended)

6) Current age as of today: (Options: <18 years old; 18-22 years old; 23-26 years old; 2730 years old; 31 years old or $>$ ).

7) Number of years between undergraduate education and starting medical school. (Open ended)

8) Undergraduate and/or other graduate institution(s), please list all (Open ended) 
Appendix 2: IRB Approval Letter

\section{WestVurginiaUniversity.}

OFFICE OF HUMAN RESEARCH PROTECTIONS

886 Chestnut Ridge Road Morgantown, WV 26506

Flex Model Approval

$03 / 04 / 2021$

To: Erin McHenry-Sorber

From: WVU Human Research Protection Program

Protocol Type: NHSR / Flex

Approval Date: 03/04/2021

Submission Type: Initial

Expiration Date: 03/03/2026

Funding: N/A

WVU Protocol \#: 2102233997

Protocol Title: The Appalachian Medical Student Experience: A Case Study

The West Virginia University Institutional Review Board has determined that your submission of protocol 2102233997 qualifies for approval under the WVU Flexibility Review Model.

- FLEX: The review of the above referenced protocol was conducted in accordance with the ethical principles of the Belmont Report. The protocol meets the criteria for approval Flex Model Review because the project does not have federal funding, is considered to be minimal risk, and falls within the guidelines of the WVU Flex Model as of this approval date.

The following documents were reviewed and approved for use as part of this submission. Only the documents listed below may be used in the research. Please access and print the files in the Notes \& Attachments section of your approved protocol.

- OHRP-47 eIC OMR No Signature Submission Worksheet.docx

- Appendix 2 Student Protocol.docx

- Appendix 1- Questionnaire for all Participants.docx

- Email Advertisement (2:26).docx

- Cover Letter Appalachian Medical Students (2:26).docx

WVU IRB approval of protocol 2102233997 will expire on 03/03/2026. 
1. There is no continuing review option for Flex protocols. If this protocol is still active after five (5) years and you intend to continue, you will need to resubmit a new protocol for approval no less than three (3) weeks prior to the expiration date in order to ensure uninterrupted study continuation.

2. Amendments are not accepted on Flex protocols and are not required as long as there is no increase to risk to participants and changes do not exclude the study from Flex inclusion criteria.

3. The IRB must be notified if there is an increase in risk to participants or if federal funding is awarded to conduct research under this protocol. In any of these cases, this protocol must be submitted as a new submission in WVU+kc.

4. Unanticipated, serious adverse events and/or side effect(s) encountered at WVU or an affiliate site that are related to the research must be reported to the WVU IRB within five (5) days using the Notify IRB action in WVU+kc.

5. Any Unanticipated Problem or UPIRTSO or other research related event resulting in new or increased risk of harm to study subjects, occurring at WVU or an affiliate site, must be reported to the WVU IRB within five (5) days using the Notify IRB action in WVU+kc.

6. All research personnel performing tasks related to the research must complete and remain current for the required training as applicable to the protocol referenced above.

7. Only Flex Consent Forms with the WVU+kc watermark may be used to consent participants.

The WVU Office of Human Research Protections will be glad to provide assistance to you throughout the research process. Please feel free to contact us by phone at 304.293 .7073 or by email at

IRB@mail.wvu.edu.

Sincerely,

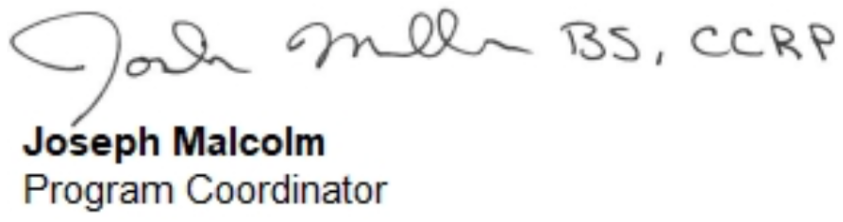


Appendix 3: Project Cover Letter

\section{WestVirginiaUniversity OFFICE OF HUMAN RESEARCH PROTECTION \\ Cover Letter Minimal Risk}

Dear Participant,

This letter is a request for you to take part in a research project that seeks to understand the experience of Appalachian medical students. This project is being conducted by Jason Hedrick, a PhD Candidate with the Department of Curriculum and Instruction/Literacy Studies at WVU under the supervision of Dr. Erin McHenrySorber, Associate Professor and Program Coordinator in the Department of Curriculum and Instruction/Literacy Studies at WVU, to fulfill requirements for a Doctorate of Philosophy in Higher Education.

If you decide to participate, you will be asked to participate in an interview through the Zoom video conferencing software. Your participation in this project will take approximately one hour. You must have spent at least a portion of your upbringing in an Appalachian county or counties as identified by the Appalachian Regional Commission. You must be 18 years of age or older to participate. Upon completion of the interview, you will be eligible to receive one Amazon gift card worth $\$ 10$.

Your involvement in this project will be kept as confidential as legally possible. All data will be reported in the aggregate. The interviews will be recorded for the researcher's references; all files will be saved on a secure, password-protected server separately from the list of participant names. Your participation is completely voluntary. You may skip any question that you do not wish to answer and you may discontinue at any time. Your class standing will not be affected if you decide either not to participate or to withdraw. West Virginia University's Institutional Review Board acknowledgement of this project is on file.

If you have any questions about this research project, please feel free to contact me at 304-642-1967 or by e-mail at jhedric8@mix.wvu.edu; Dr. McHenry-Sorber can be reached via email at ecmchenrysorber@mail.wvu.edu. If you have any questions about your rights as a research participant, please contact the WVU Office of Human Research Protection by phone at 304-293-7073 or by email at IRB@mail.wvu.edu.

I hope that you will participate in this research project, as it could help us better understand the transition and associated challenges that students from Appalachia experience while moving through the medical education experience. Thank you for your time and consideration.

Sincerely,

\section{Jasan S. Hedrick}

Jason S. Hedrick

PhD Candidate

Chestnut Ridge Research Building

Phone: 304-293-7073 886 Chestnut Ridge Road

Fax: 304-293-3098 PO Box 6845

http://oric.research.wvu.edu Morgantown, WV 26506-6845
$\mathrm{P}$ a g e $\mid \mathbf{1}$

V2019.06.03 


\section{Appendix 4: Student Protocol}

1) Can you describe what the word "Appalachia" means to you?

a. Probe: How much or in what ways do you identify as Appalachian?

2) What do you think are the biggest challenges facing the Appalachian region?

a. Probe: What challenges are facing your home community?

3) Can you describe your upbringing in Appalachia?

a. Probe: What was your relationship with your immediate and extended family like?

b. Probe: What are the schools (high school) like that you attended?

4) When did you decide that you wanted or were going to attend college?

a. Probe: What was the transition like from home to college?

b. Probe: At any point did you consider how attending college may change your relationship with your home?

c. Probe: Who or what were motivated you to go to college?

i. Did anyone try to dissuade you from going to college?

5) When and why did you decide to go to medical school?

a. Probe: Can you think of any individuals who motivated your desire to go medical school?

b. Probe: Did any individuals dissuade you or question your desire to attend medical school?

6) Where did you complete community/rural rotations and requirements?

a. Probe: What do you think are the benefits of completing a community and rural rotation prior to graduation? 
b. Probe: What did you think about your experience during those rotations?

7) After you complete your medical degree, what are your plans?

a. Probe: Do you plan on practicing in a rural area? How close is this to your home?

b. Probe: How or did any individuals (e.g., faculty, peers) at the medical school influence your decision to stay or leave?

c. Probe: Did specialization opportunities influence your decision?

d. Probe: How did your performance in medical school (such as metrics) influence your decision to stay or leave?

8) If the participant desires to return home for future practice:

a. What are your biggest motivations for returning home to practice?

b. Are there any barriers for returning home to practice medicine?

9) If the participant DOES NOT desire to return home for future practice:

a. What are your biggest motivations for practicing outside of the region that you grew up?

b. Are there any barriers for returning home to practice medicine? 